\title{
Energy Exchange between Unidirectional Vehicle-To-Grid Aggregators, and Wind and Conventional Generating Companies in the Electricity Market
}

\author{
by
}

Ahmad Tavakoli

Submitted in fulfilment of the requirements for the Degree of Doctor of Philosophy

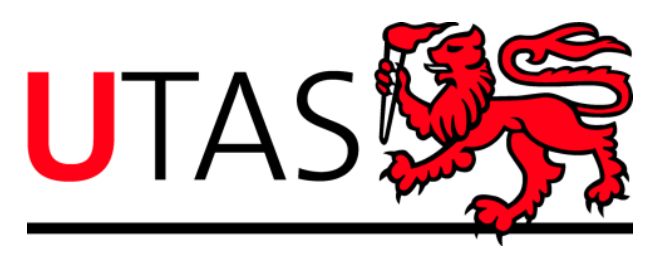

School of Engineering

University of Tasmania

June 2016 
to $m$.. 


\section{Declaration of Originality}

This thesis contains no material which has been accepted for a degree or diploma by the University or any other institution, except by way of background information and duly acknowledged in the thesis. To the best of my knowledge and belief, this thesis contains no material previously published or written by another person except where due acknowledgement is made in the text of the thesis, nor does the thesis contain any material that infringes copyright.

Ahmad Tquakoli
Date: $15 / 06 / 2016$

\section{Statement of Authority to Access}

This thesis may be available for loan and limited copying in accordance with the Copyright Act 1986.
Ahmad Tolvakoli
Date: $15 / 06 / 2016$ 


\section{Statement of Co-Authorship}

The following people and institution contributed to the publication of work undertaken as part of this thesis:

Name and school: Ahmad Tavakoli, School of Engineering and ICT.

Name and institution: Prof Michael Negnevistky, School of Engineering and ICT, UTAS.

Name and institution: Dr. Kashem Mutaqqi, the University of Wollongong.

Name and institution: Dr. Duy Thanh Nguyen, the TasNetwork Company.

Paper 1, "Energy Exchange between Electric Vehicle Load and Wind Generating Utilities"

Candidate was the primary author and with author $2(10 \%)$, author $3(5 \%)$, and author $4(5 \%)$ contributed to its refinement and development.

Paper 2, "Pool Strategy of a Producer Coordinated with Vehicle-to-Grid Services to Maximize Profitability".

Candidate was the primary author and with author $2(10 \%)$, and author $3(10 \%)$ contributed to its refinement and development.

Paper 3, "A Coordinated Approach to Energy Exchange between Electric Vehicle Load Aggregators and Wind Generation Companies under Uncertainty".

Candidate was the primary author and with author $2(10 \%)$, and author $3(10 \%)$ contributed to its refinement and development.

We the undersigned agree with the above stated "proportion of work undertaken" for each of the above published (or submitted) peer-reviewed manuscripts contributing to this thesis.

Signed:

Supervisof's name: Michael Negnevistky

School of Engineering and ICT

University if Tasmania

Date: $26,04,16$
Signed:,

Head of School

School of Engineering and ICT

University if Tasmania

Date: $26^{\mathrm{k}} \mathrm{Afmi}^{\prime}(20<6$ 


\section{Abstract}

The future of humanity is dependent on saving the environment from global warming caused by $\mathrm{CO} 2$ emission from electricity generation and transportation systems. The remedies are the increasing in the penetration of renewable energy in electricity generation and electric vehicles $(\mathrm{EVs})$ in transportation. The main operational problem associated with a high wind penetration and EVs comes from intermittency and unpredictability. The power systems are likely to face increasing uncertainties in both generation and load sides and there is no coordination between them. In addition, EVs might impose excessive load on the grid. Therefore, coordinating the EV aggregator with the generating companies in the electricity market can enhance the stability of the power system via unidirectional vehicle-to-grid (V2G) technology.

This thesis concentrates on the impact of the participation of the EV load aggregator and wind power, and the coordination strategy on the market outcomes and prices.

Firstly, power exchange between the wind generating companies (WGenCos) and EV load aggregators considered as price-takers in the energy and ancillary service markets is modelled and analysed. A two-stage stochastic linear programming-based optimal offering/bidding strategy model is developed for the coordinated EV-Wind units participating in the day-ahead energy, balancing, and regulation markets. In future electricity markets, the EV aggregator will have a more important role with high penetration of EV numbers. Finally, the EV aggregator as price-maker which is in generation portfolio of single and multiple strategic firms including WGenCo and conventional generating companies (CGenCos) is modelled and investigated. A stochastic optimal bidding/offering strategy is developed for the EV load aggregator providing the energy and ancillary services in coordination with single and multiple strategic firms in a pool-based electricity market with endogenous formation of day-ahead and realtime prices, and EV aggregator tariff.

The methodology consists of using stochastic optimization categorized into single and multiple optimization problems. In the single optimization problem, WGenCo and EV aggregator considered as price-takers aim to maximize their objective function associated with equality or inequality constraints. In multiple optimization problems, the strategic firms such as WGenCos, EV aggregators, and other players considered as price-makers, submit supply-offers/demand-bids 
to the market operator to participate in the electricity market. A bilevel (hierarchical) model is used in this thesis to model the behaviour of each player. A bilevel problem includes an upper-level problem and a set of lower-level problems which are limited by the upper and lower equality and inequality constraints.

Throughout the thesis, both analytical proofs and numerical examples are provided to review the market analysis of EV aggregator, CGenCo and WGenCo and the coordination strategy.

The numerical results show the effectiveness of the coordination strategy, which is profitable and beneficial with increasing EV penetration in comparison with the incoordination strategy. We conclude that EV aggregators as an individual firm could not compete with other conventional, dispatchable companies. Hence, merging EV aggregators in CGenCos' and WGenCos' portfolio would increase the payoff of EV aggregators and strategic firms. However, a sufficient EV number is a significant factor to affect market and EV aggregator outputs. Moreover, the numerical results show that the EV tariff and numbers at EV-level can influence the market price and power generation at wholesale-level in the electricity market. In addition, the high penetration of EVs leads to increasing the wind power penetration and reducing the wind power curtailment. 


\section{Publications}

The following is a list of journal and conference papers which have been produced as an outcome of the $\mathrm{PhD}$ candidate's research.

1. Tavakoli, M. Negnevitsky, D. T. Nguyen, K. Muttaqi, "Energy Exchange between Electric Vehicle Load and Wind Generating Utilities", IEEE Transactions on Power Systems, vol. 31, no. 2, pp. 1248-1258, March 2016.

2. Tavakoli, M. Negnevitsky, K. Muttaqi," Pool Strategy of a Producer Coordinated with Vehicle-to-Grid Services to Maximize Profitability", Power Engineering Conference (AUPEC), 2015 Australasian Universities, Wollongong, NSW, 2015, pp. 1-6.

3. Tavakoli, M. Negnevitsky, K. Muttaqi," A Coordinated Approach to Energy Exchange between Electric Vehicle Load Aggregators and Wind Generation Companies under Uncertainty", 2015 IEEE Power \& Energy Society General Meeting, Denver, CO, 2015, pp. 1-5.

4. J. Hamilton, A. Tavakoli, M. Negnevitsky, X. Wang, S. Gamble, "No Load Diesel Application to Maximize Renewable Energy Penetration in Off grid Hybrid Systems" 2016 Paris session, Cigre.

5. A. Tavakoli, Negnevitsky, M., and Kashem, M., “A Decentralized Model Predictive Control for Operation of Multiple Distributed Generators in Islanded Mode", Revised at the IEEE Transactions on Industry Applications.

6. James Hamilton, A. Tavakoli, Michael Negnevitsky, Xiaolin Wang, "Investigation of No Load Diesel Technology in Isolated Power Systems" Accepted at 2016 IEEE Power \& Energy Society General Meeting.

7. A. Tavakoli, Negnevitsky, M., Lyden, S. and Haruni A.M.O., "A Decentralized Control Strategy for Multiple Distributed Generation in Islanded Mode", Proceedings of the IEEE/PES General Meeting, Washington DC, USA, 27-31 July, 2014, IEEE Catalog Number CFP14POW-USB.

8. A. Tavakoli, M. Negnevitsky, "A Novel Control Strategy for a Distributed Generation (DG) in Islanding Mode," Published at the AUPEC 2013, 29 September - 3 October 2013. 


\section{Acknowledgements}

First of all, I am deeply grateful to my mother for her selfless devotion, and my father and sister for their support during all my life.

I would like to express my deep gratitude to my supervisor, Prof. Michael Negnevitsky, for his encouragement, and belief in my abilities. I have been fostered productively through involvement in his diverse industrial research projects with different stakeholders. I would also like to thank Dr. Kashem Mutaqqi for his productive comments.

In the School of Engineering and ICT at UTAS, I would like to acknowledge all the academic staff, especially Dr. Bernardo A. León de la Barra, Dr. Enamul Haque, and Dr. Sarah Lyden, for their support over the years. I also appreciate the general staff, especially Mary Dixon, Bernard Chenery, and Calverly Gerard, who particularly supported me with all necessary equipment and facilities for my research.

I thank all of my colleagues and friends, especially Zane Smith, Omid Sadeghi, Behzad Naderi, and James Hamilton, with whom I have shared significant knowledge and experience during the course of my student life. A very special thank you goes to Osman Haruni for not only career orientation but also for providing much helps since the first days I arrived in Hobart.

And finally, but not less important, I would like to thank to Dr. Tim Gale for his constructive position as the graduate coordinator in the School of Engineering and ICT at UTAS. 


\section{Contents}

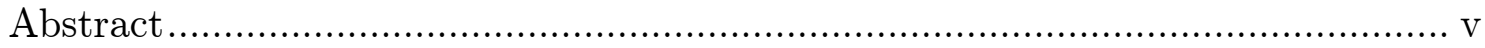

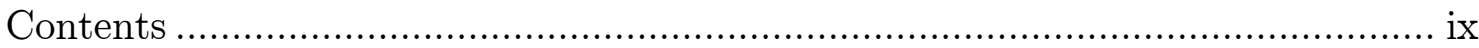

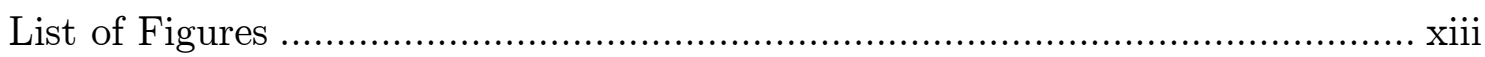

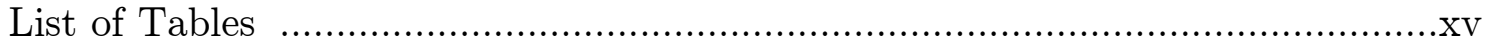

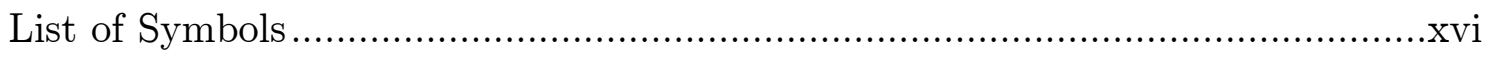

List of Abbreviations and Acronyms..............................................................xiv

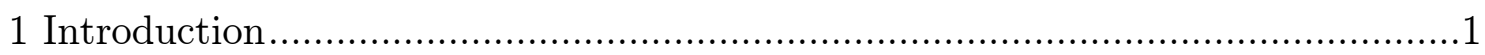

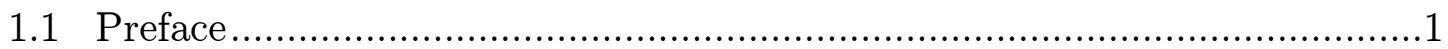

1.2 Review of Current Trends in Integrating Electric Vehicles into Grids .........3

1.2.1 Common Modelling Assumptions....................................................5

1.3 Mathematical Structures of Electricity Markets .................................. 9

1.4 Project Objectives ........................................................................... 12

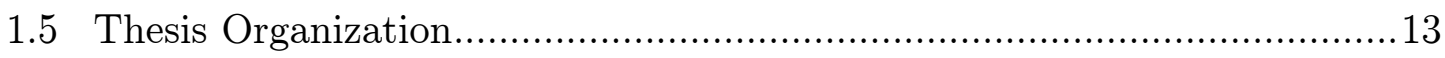

2 Electric Vehicles in Electricity Markets ......................................................... 17

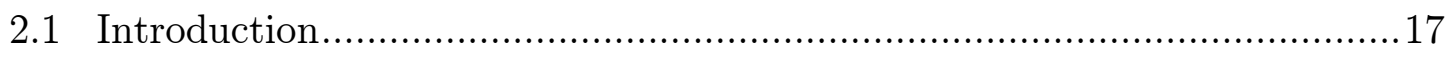

2.2 Electricity Markets .............................................................................. 18

2.2.1 Pool-based Electricity Market................................................. 19

2.2.2 Reserve and Regulation Markets..................................................20

2.2.3 The Futures Market and Bilateral Contracts ..............................21

2.3 Uncertainty in the Power Grid ..........................................................21

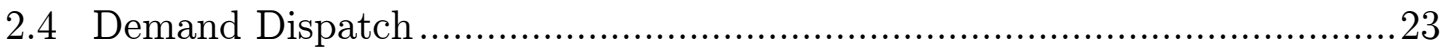

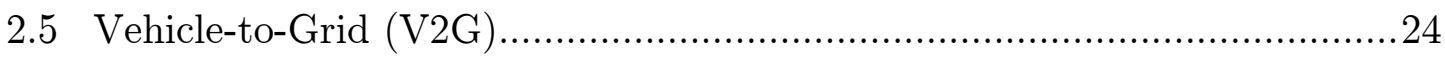

2.6 Regulation Ancillary Services via Unidirectional V2G..........................25

2.7 Mathematical Formulation of Unidirectional V2G in the Electricity Market..... 
3 Energy Exchange between Electric Vehicle Load and Wind Generating

Utilities

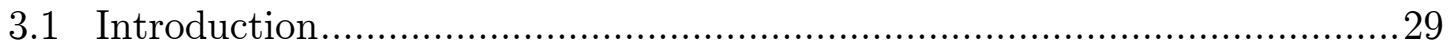

3.2 Market Framework .............................................................................. 31

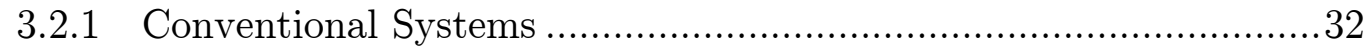

3.2.2 WGenCo with the Energy Storage System ................................. 32

3.2.3 Coordinated EV-Wind Energy Exchange...................................... 33

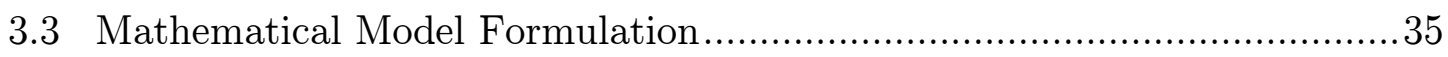

3.3.1 Conventional Systems …....................................................... 36

3.3.2 WGenCo with the Energy Storage System ................................. 37

3.3.3 Coordinated EV-Wind Energy Exchange......................................39

3.4 Case Studies and Numerical Results .....................................................42

3.4.1 Payoff Analysis..................................................................... 44

3.4.2 Demand and Generation Dispatch analysis ..............................4 49

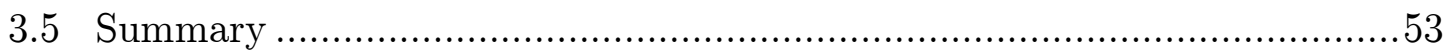

4 Pool Strategy of a Single Firm in Coordination with EV Load Aggregators ....55

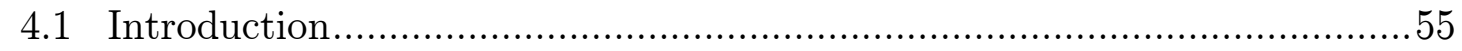

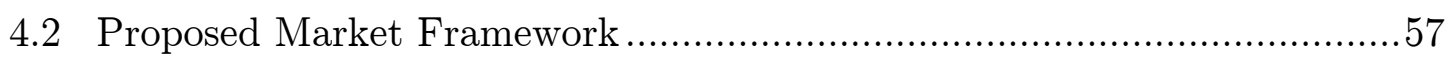

4.3 Mathematical Model Formulation ...................................................... 60

4.3.1 Bilevel Model................................................................ 60

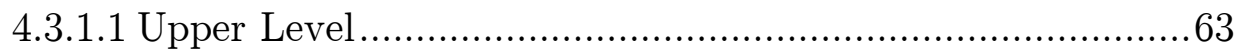

4.3.1.2 Day - Ahead Lower Level .............................................64

4.3.1.3 Real -Time Lower Level ................................................65

4.3.1.4 EV Energy Lower Level ...............................................67

4.3.2 Mathematical Program with Equilibrium Constraints ................67

4.3.2.1 KKT Conditions Corresponding to the Day-ahead Lower

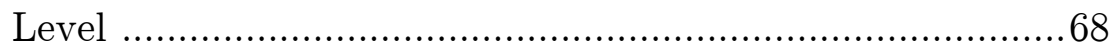

4.3.2.2 KKT Conditions Corresponding to the Real-time Lower Level 69 
4.3.2.3 KKT Conditions Corresponding to the EV Lower Level ....72

4.3.2.4 Strong Duality Theorem Corresponding to the Day-ahead .73

4.3.2.5 Strong Duality Theorem Corresponding to the Real-time

Lower Level Lower Level ................................................73

4.3.2.6 Strong Duality Theorem Corresponding to the EV Lower

Level.................................................................. 74

4.3.3 Mixed-Integer Linear Programming ........................................ 75

4.3.3.1 Linearization of Complementarity Conditions ...................75

4.3.3.2 Linearization of Nonlinear terms...................................... 80

4.4 Case Studies and Numerical Results ......................................................8 81

4.4.1 Payoff and Price Analysis .................................................... 83

4.4.2 Demand and Generation Dispatch Analysis ...............................88

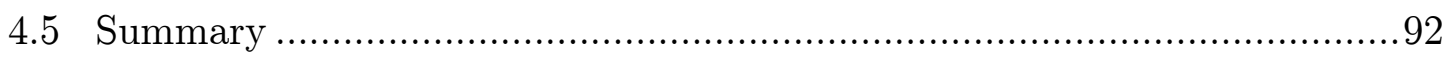

5 Pool Strategy of Multiple Firms in Coordination with EV Load Aggregators .93

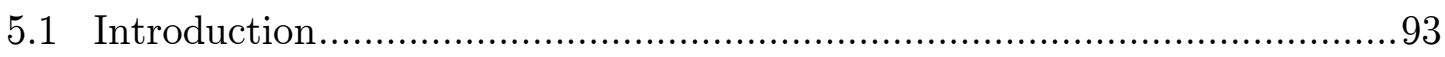

5.2 Market Framework and Approach ......................................................... 94

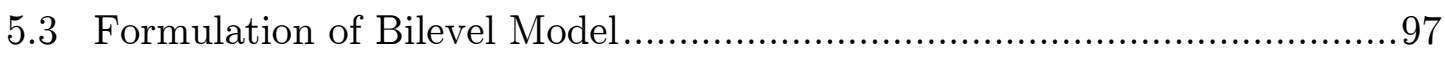

5.3.1 Mathematical Program with Equilibrium Constraints.................. 99

5.3.2 Equilibrium Problem with Equilibrium Constraints .................. 102

5.3.3 Mixed-Integer Linear Programming ...................................... 112

5.3.3.1 Linearization of Nonlinear Terms in the Strong Duality Equalities................................................................... 112

5.3.3.2 Linearization of Complementarity Conditions ................. 114

5.3.3.3 Linearization of Nonlinear Terms Comprising $\emptyset_{j}^{E V}, \emptyset_{j, s}^{R T}$, and

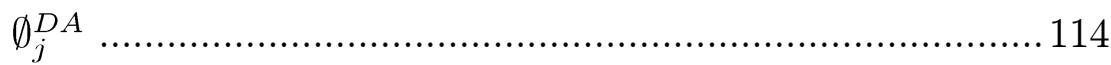

5.3.4 Diagonalization Algorithm ............................................... 115

5.4 Case Studies and Numerical Results …........................................ 116

5.4.1 Payoff and Price Analysis ................................................. 117

5.4.2 Demand and Generation Dispatch Analysis ............................. 119 


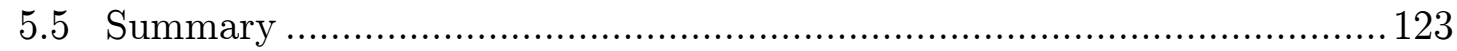

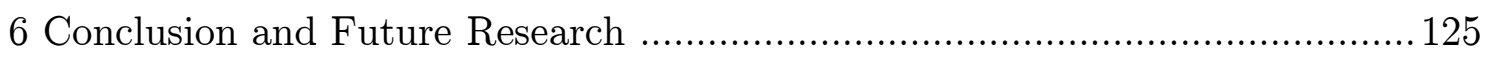

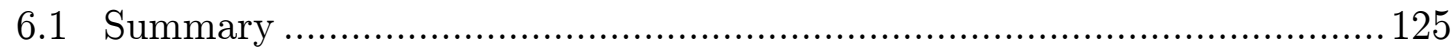

6.1.1 Coordinating EV load aggregators and WGenCos

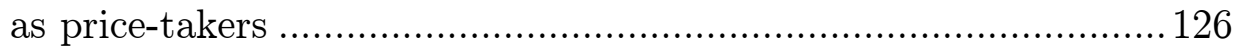

6.1.2 Coordinating EV load aggregators and all GenCos

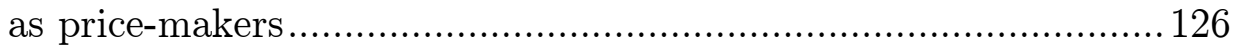

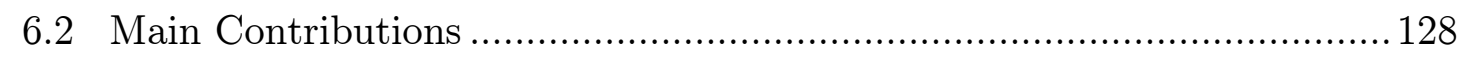

6.3 Recommendations for Future Research …………..............................129

Appendix A Scenario Generation and Reduction ……………......................... 131

A.1 Wind and Energy Price Scenarios ............................................................. 131

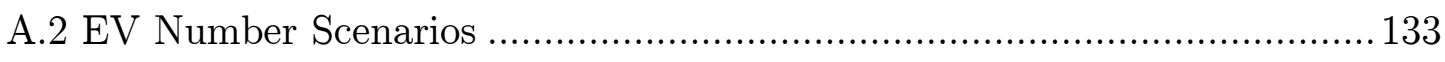

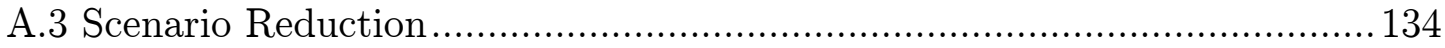

Appendix B Absolute Value of the Variable in the MILP ..................................137

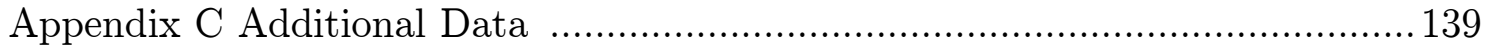

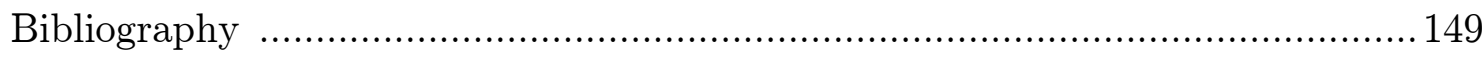




\section{List of Figures}

1.1 Optimization problems

Fig. 1.2 Bilevel model formulated as a single-level stochastic MPEC

Fig. 1.3 An Equilibrium Problem with Equilibrium Constraints (EPEC).....

Fig. 2.1 The structure of a pool-based electricity market

Fig. 2.2 Bilateral contracting of electricity

Fig. 2.3 The clearing times of future, day-ahead, reserve, regulation, and balancing (real-time) markets

Fig. 2.4 EV aggregator regulating; the shaded area highlights the energy drawn from the grid

Fig. 3.1 Coordination between EV demand and wind power deviation in energy and regulation market

Fig. 3.2 The intra-hourly wind power generation forecasted for ten scenarios. ....43

Fig. 3.3 The day-ahead energy price, regulation up/down prices, and intra-hour real time energy price scenarios

Fig. 3.4 The intra-hourly EV penetration forecasted for ten scenarios

Fig. 3.5 The WGenCo's payoff versus penalty prices

Fig. 3.6 Imbalance charges provided by balancing market versus penalty prices .48

Fig. 3.7 EV regulation and battery discharging cost versus penalty prices

Fig. 3.8 Wind power generation and EV demand schedule in Case D under the $\$ 10 /$ MWh penalty price.

Fig. 3.9 Wind power generation and EV demand schedule in Case D under the $\$ 150 /$ MWh penalty price.

Fig. 3.10 Wind power deviation, battery energy storage profile in case B at 10 $\$ /$ MWh penalty price

Fig. 3.11 Wind power generation schedule, and battery energy storage profile in case $\mathrm{B}$ at $150 \$ / \mathrm{MWh}$ penalty price.

Fig. 4.1 The proposed wholesale \& EV-levels structure of the electricity market (the firm's components are within the boundary of the broken lines) 
Fig. 4.2 Bilevel structure of the proposed wholesale \& EV-levels structure of the electricity market

Fig. 4.3 Interrelation between the upper-level and lower level problems 62

Fig. 4.4 Demand bid prices in five demand blocks 82

Fig. 4.5 The intra-hourly wind power generation forecasted for ten scenarios. ....82

Fig. 4.6 The intra-hourly EV penetration forecasted for ten scenarios ...............82

Fig. 4.7. Impact of EV numbers and tariffs on WGenCo profit 85

Fig. 4.8. Impact of EV numbers and tariffs on the total profit 85

Fig. 4.9. Impact of fixed-rate tariff and 50,000 EVs on the day-ahead price ......86

Fig. 4.10. Impact of ToU tariff and 50,000 EVs tariff on the day-ahead price ...86

Fig. 4.11. Impact of fixed-rate tariff and 100,000 EVs on the day-ahead price ..87

Fig. 4.12. Impact of ToU tariff and 100,000 EVs on the day-ahead price 87

Fig. 4.13. Impact of fixed-rate tariff and 50,000 EVs on generation and demand dispatch .89

Fig. 4.14. Impact of ToU tariff and 50,000 EVs on generation and demand dispatch

Fig. 4.15. Impact of fixed-rate tariff and 100,000 EVs on generation and demand dispatch

Fig. 4.16. Impact of ToU tariff and 100,000 EVs on generation and demand dispatch

Fig. 4.17. Impact of tariffs and EV numbers on total demand and CGenCo power generation

Fig. 4.18. Impact of tariffs and EV numbers on wind power generation 91

Fig. 4.19. Impact of tariffs and EV numbers on wind curtailment .91

Fig. 5.1 The proposed wholesale \& EV-levels structure of the electricity market for multiple strategic firms .95

Fig. 5.2 The steps to obtain the market equilibria 96

Fig. 5.3 A one-iteration diagonalization algorithm 115

Fig. A.1 A path through the tree is called a scenario and consists of realizations of all random variables. 


\section{List of Tables}

Table 3.1 WGenCo's Payoffs In Different Cases at the $\$ 30$ Penalty Price...........45

Table 3.2 Impact of the Penalty Price on WGenCo's Payoffs In Case A .............47

Table 3.3 Impact of the Penalty Price on WGenCo's Payoffs In Case B .............47

Table 3.4 Impact of the Penalty Price on WGenCo's Payoffs In Case D ............47

Table 3.5 Total Real-Time Wind Power Generation, Wind Power Deviation and

Energy Imbalance per Day ...................................................................... 50

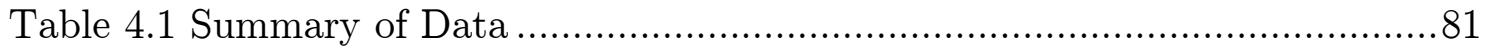

Table 4.2 EV Aggregator's Profit...................................................... 85

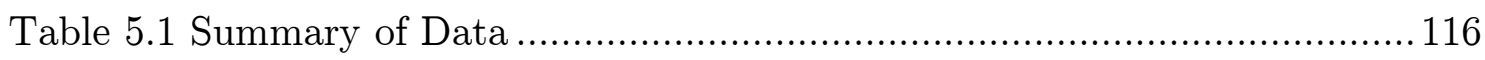

Table 5.2 The Combination of WGenCos, CGenCos, and EV Aggregators in

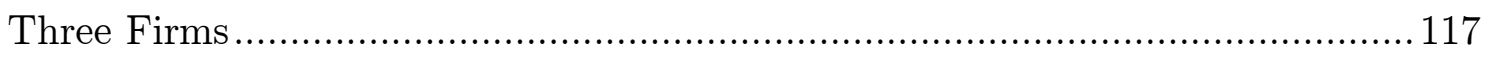

Table 5.3 Firms' Payoff in Different Cases ....................................................... 118

Real-time Wind Power Deviations and Regulation up/down of the Units for $200,000 \mathrm{EVs}$

Table 5.5 Demand Curtailment and Wind Power Curtailment for 100,000 EVs 121

Table 5.6 Demand Curtailment and Wind Power Curtailment for 100,000 EVs 122

Table 5.7 Regulation up/down of the EV aggregators and Demand Curtailment

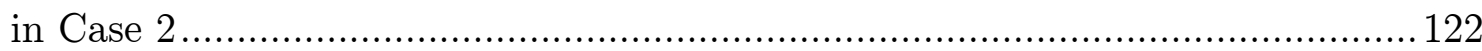

Table 5.8 Regulation up/down of the EV aggregators and Demand Curtailment

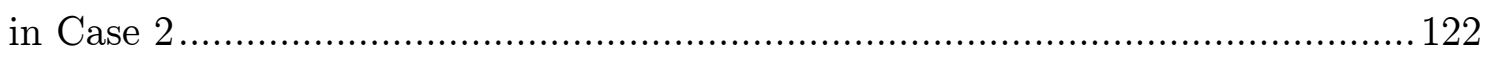

Table A.1 The Availability of EVs in Various Time Intervals ........................ 134

Table C.1 The Results of Profit and Prices for Case 1 ................................ 140

Table C.2 The Results of Profit and Prices for Case 2 ................................. 141

Table C.3 The Results of Profit and Prices for Case 3 ................................. 142

Table C.4 The Results of Profit and Prices for Case 4 ................................... 143

Table C.5 The Results of Demand and Generation Dispatch for Case 1........... 144

Table C.6 The Results of Demand and Generation Dispatch for Case 2.......... 145

Table C.7 The Results of Demand and Generation Dispatch for Case 3........... 146 


\section{List of Symbols}

\section{Indices:}

$\begin{array}{ll}b & \text { Battery storage bank } \\ d & \text { Demands. } \\ e v & \text { Electric vehicle fleet. } \\ g & \text { Conventional generating units. } \\ i & \text { Intra-hour (sub-hour) time intervals } \\ j & \text { Firms from 1 to J. } \\ m & \text { A segment of curves } \\ s & \text { Scenarios } \\ t & \text { Hourly time intervals } \\ w & \text { Wind energy units } \\ y & \text { Index for firms from } 1 \text { to Y. }\end{array}$

\section{Parameters:}

$C_{g}$

$C_{d, t}^{L}$

$D_{e v, s, t, i}$

$E_{e v, t}^{\max }, E_{e v, t}^{\min }$

$N_{e v}$

$N_{e v, s, t, i}$

$N_{I}$

$N_{s}$
Marginal cost of conventional units (\$/MWh).

Load curtailment cost $(\$ / M W h)$.

Energy consumption by EV while driving.

Max/Min available energy in EV aggregator.

Number of EV fleet.

Plugged-in EV number scenarios in each fleet

Number of intrahour intervals.

Number of scenarios. 


\begin{tabular}{|c|c|}
\hline$N_{T}$ & Number of hour intervals. \\
\hline$N_{W}$ & Number of wind energy units. \\
\hline$P_{g}^{\max }$ & Maximum power generation of CGenCo (MW). \\
\hline$P_{w}^{\max }$ & Maximum power generation of WGenCo (MW). \\
\hline$P_{e v}^{\max }$ & Maximum power drawn of EV aggregator (MW). \\
\hline$P_{w, t, i}^{R T^{s}}$ & Real-time forecasted wind power (MW). \\
\hline$P_{b, \text { Max }}^{c h}, P_{b, \text { Max }}^{d c}$ & Max charge/discharge power of the battery storage bank. \\
\hline$P_{e v, c h}^{\max }$ & Max charge power of the EV aggregator. \\
\hline$P_{s, t, i}^{w, f}$ & Intra-hour forecasted wind power (MW). \\
\hline$P_{t}^{w, f}$ & Day-ahead forecasted wind power (MW). \\
\hline$L_{d}^{\max }$ & Max load demand (MW). \\
\hline$R_{e v}^{u p^{\max }}, R_{e v}^{d^{\max }}$ & Max regulation up/down of EV aggregator (MW). \\
\hline$R_{g}^{u p^{\max }}, R_{g}^{d^{\max }}$ & Max regulation up/down of CGenCo (MW). \\
\hline$S O C_{e v}^{\max , \min }$ & Maximum/Minimum state of the charge. \\
\hline $\mathrm{T}$ & Time at which specified state of charge is adjusted. \\
\hline$i 0, N I$ & $\begin{array}{l}\text { The beginning and the end of each intra-hour time } \\
\text { interval. }\end{array}$ \\
\hline$\rho_{t}^{D A, f}$ & Forecasted day-ahead energy price $(\$ / \mathrm{MWh})$. \\
\hline$\rho_{s, t, i}^{R T, f}$ & Forecasted real-time energy price $(\$ / \mathrm{MWh})$. \\
\hline$\rho_{t}^{R u p}$ & Day-ahead regulation-up price $(\$ / \mathrm{MWh})$. \\
\hline$\rho_{t}^{\text {Rdown }}$ & Day-ahead regulation-down price (\$/MWh). \\
\hline$\rho^{P}$ & Penalty price $(\$ / M W h)$. \\
\hline$\rho^{T, f}$ & Forecasted EV charging tariff (\$/MWh). \\
\hline$\beta_{d, t}^{D A}$ & Day-ahead bid price of LSE (\$/MW) \\
\hline$\pi_{s}$ & Probability of scenarios. \\
\hline$\varphi_{m}$ & Slope of segment in linearized charge / discharge curve. \\
\hline$\Delta t$ & Duration of each intra-hour time interval. \\
\hline
\end{tabular}




\section{Variables:}

\begin{tabular}{|c|c|}
\hline$a_{s, t, k}^{\Delta}, b_{s, t, k}^{\Delta}$ & Auxiliary binary variables. \\
\hline$\varphi_{s}, \gamma$ & Auxiliary variables for computing $\mathrm{CVaR}$ \\
\hline$\rho_{t}^{D A}$ & Day-ahead clearing price $(\$ / \mathrm{MWh})$. \\
\hline$\rho^{T}$ & EV charging tariff $(\$ / \mathrm{MWh})$. \\
\hline$\rho_{t, i, s}^{R T}$ & Real-time clearing price ( $\$ / \mathrm{MWh})$. \\
\hline$\alpha_{g, t}^{D A} \alpha_{g, t, i}^{R T^{s}}$ & Day-ahead/Real-time offer price of CGenCos $(\$ / \mathrm{MW})$. \\
\hline$\alpha_{w, t}^{D A} \alpha_{w, t, i}^{R T^{s}}$ & Day-ahead/Real-time offer price of WGenCos $(\$ / \mathrm{MW})$. \\
\hline$\alpha_{e v, t, i}^{R T^{s}}$ & Real-time offer price of EV aggregator $(\$ / M W)$. \\
\hline$\beta_{e v, t}^{D A}$ & Day-ahead bid price of EV aggregator $(\$ / \mathrm{MW})$ \\
\hline$C_{b, s, t, i}^{d c}$ & Degradation cost of the discharging battery. \\
\hline$E_{e v, t, i}^{s}$ & Real-time energy of EV aggregator. \\
\hline$I_{b, s, t, i}^{c h}$ & Charging indicator of the battery storage. \\
\hline$I_{b, s, t, i}^{d c}$ & Discharging indicator of the battery storage. \\
\hline$I_{e v, t, i}^{u p^{s}}$ & Regulation up indicator of the EVs. \\
\hline$I_{e v, t, i}^{d o w n^{s}}$ & Regulation down indicator of the EVs. \\
\hline$L_{d, t}^{D A}$ & Day-ahead demand (MW). \\
\hline$L_{d, t, i}^{C^{s}}$ & Demand load curtailment (MW). \\
\hline$P F_{s}^{\mathrm{CGenCo}}$ & The expected profit of CGenCos $(\$)$. \\
\hline$P F_{s}^{\mathrm{WGenCo}}$ & The expected profit of WGenCos $(\$)$. \\
\hline$P F_{s}^{\mathrm{Agg}}$ & The expected profit of EV aggregator $(\$)$. \\
\hline$P_{g, t}^{D A}$ & Day-ahead power generations of CGenCos (MW). \\
\hline$P_{w, t}^{D A}$ & Day-ahead power generations of WGenCos (MW). \\
\hline$P_{w, t, i}^{C^{s}}$ & Wind power curtailment (MW). \\
\hline$P_{o} P_{e v, t}$ & Preferred operating point (day-ahead power-drawn) of \\
\hline
\end{tabular}


$P_{b, s, t, i}^{c h}$

$P_{e v, s, t, i}^{c h}$

$P_{b, s, t, i}^{d c}$

$P_{b, m, s, t, i}^{d c}$

$P_{s, t, i}^{i m}$

$P_{|i m|, s, t, i}^{i m}$

$R_{g, t, i}^{u p^{s}}$

$R_{g, t, i}^{d o w n^{s}}$

$R_{e v, t, i}^{u p^{s}}$

$R_{e v, t, i}^{d o w n^{s}}$

$\Delta P_{|\Delta| s, t, i}^{w}$

$\Delta P_{s, t, i}^{w}$ the EVs (MW).

Charge power of the battery storage bank (MW).

Real-time power drawn by the EV aggregator (MW).

Discharge power of the battery storage bank (MW).

Discharge power of the battery storage at segment $m$ (MW).

Energy imbalance adjusted (provided) by the balancing market (MW).

Absolute imbalance power of balancing market

Regulation-up power of CGenCos power (MW).

Regulation-down power of CGenCos power (MW).

Regulation-up power of EV aggregator power (MW).

Regulation-down power of EV aggregator power (MW).

Absolute wind power deviation between Real time and day-ahead scheduling (MW).

Wind power deviation between Real time and day-ahead scheduling (MW).

\section{Dual variables}

$$
\begin{aligned}
& \mu_{g, t}^{D A^{\text {max }}}, \mu_{g, t}^{D A^{\text {min }}} \\
& \mu_{e v, t}^{D A^{\text {max }}}, \mu_{e v, t}^{D A^{\text {min }}} \\
& \mu_{w, t}^{D A^{\text {max }}}, \mu_{w, t}^{D A^{\text {min }}} \\
& \mu_{d, t}^{D A^{\text {max }}}, \mu_{d, t}^{D A^{\text {min }}} \\
& \mu_{e v, t, i, s}^{u p^{\text {max }}}, \mu_{e v, t, i, s}^{u p^{\text {min }}} \\
& \mu_{e v, t, i, s}^{u p}
\end{aligned}
$$

Upper/lower bound for day-ahead power generations of CGenCos.

Upper/lower bound for day-ahead POP of EV aggregator.

Upper/lower bound for day-ahead power generations of WGenCos.

Upper/lower bound for day-ahead load demand of LSE.

Upper/lower bound for regulation-up power of EV aggregator power.

Upper bound for real-time power of EV aggregator. 
$\mu_{e v, t, i, s}^{d_{\max }}, \mu_{e v, t, i, s}^{d^{m i n}}$

$\mu_{e v, t, i, s}^{\text {down }}$

$\mu_{g, t, i, s}^{u p^{\max }}, \mu_{g, t, i, s}^{u p^{\min }}$

$\mu_{g, t, i, s}^{u p}$

$\mu_{g, t, i, s}^{d_{\max }}, \mu_{g, t, i, s}^{d^{\min }}$

$\mu_{g, t, i, s}^{\text {down }}$

$\mu_{w, t, i, s}^{c_{\max }}, \mu_{w, t, i, s}^{c^{\min }}$

$\mu_{d, t, i, s}^{c_{\max }}, \mu_{d, t, i, s}^{c^{\min }}$

$\mu_{e v, t}^{E E V^{\max }}, \mu_{e v, t}^{E E V^{m i n}}$

$Z \alpha_{g, j}^{D A}$

$Z \alpha_{w, j}^{D A}$

$Z \beta_{e v, j}^{D A}$

$Z \alpha_{g, j}^{R T^{s}}$

$Z \alpha_{w, j}^{R T^{s}}$

$Z \alpha_{e v, j}^{R T^{s}}$

$Z_{e v, j}^{D A^{\max }}, Z_{e v, j}^{D A^{\min }}$

$Z_{g, j}^{D A^{\max }}, Z_{g, j}^{D A^{\min }}$
Upper/lower bound for regulation-down power of EV aggregator power.

Lower bound for real-time power of EV aggregator.

Upper/lower bound for regulation-up power of CGenCos power.

Upper bound for real-time power of CGenCos.

Upper/lower bound for regulation-down power of CGenCos power.

Lower bound for real-time power of CGenCos.

Upper/lower bound for wind power curtailment of WGenCos.

Upper/lower bounds for load demand curtailment of LSE

Upper/lower bounds for EV energy constraint of EV aggregator.

Dual constraint for day-ahead offer price of CGenCos in the MPEC of firm $j$.

Dual constraint for day-ahead offer price of WGenCos in the MPEC of firm $j$.

Dual constraint for day-ahead bid price of EV aggregator in the MPEC of firm $j$.

Dual constraint for real-time offer price of CGenCos in the MPEC of firm $j$.

Dual constraint for real-time offer price of WGenCos in the MPEC of firm $j$.

Dual constraint for real-time offer price of EV aggregator in the MPEC of firm $\mathrm{j}$.

Upper/lower dual constraints for day-ahead POP of EV aggregator in the MPEC of firm $\mathrm{j}$.

Upper/lower dual constraints for day-ahead power generations of CGenCos in the MPEC of firm $j$. 


$$
\begin{aligned}
& Z_{w, j}^{D A^{\max },} Z_{w, j}^{D A^{m i n}} \\
& Z_{d, j}^{D A^{\max },} Z_{d, j}^{D A^{m i n}} \\
& Z P O P_{e v, j} \\
& Z P_{g, j}^{D A}
\end{aligned}
$$$$
Z P_{w, j}^{D A}
$$$$
Z L_{d, j}^{D A}
$$$$
X_{e v, j}^{D A^{\min }}, X_{e v, j}^{D A^{\max }}
$$$$
X_{g, j}^{D A^{\min }}, X_{g, j}^{D A^{\max }}
$$$$
X_{w, j}^{D A^{\min }}, X_{w, j}^{D A^{\max }}
$$$$
X_{d, j}^{D A^{\min }}, X_{d, j}^{D A^{\max }}
$$$$
Z_{e v, s, j}^{u p^{\max }}, Z_{e v, s, j}^{u p^{\min }}
$$$$
Z_{e v, s, j}^{u p}
$$$$
Z_{e v, s, j}^{d_{\max }}, Z_{e v, s, j}^{d^{\min }}
$$$$
Z_{e v, s, j}^{\text {down }}
$$$$
Z_{g, s, j}^{u p^{\max }}, Z_{g, s, j}^{u p^{\min }}
$$

Upper/lower dual constraints for day-ahead power generations of WGenCos in the MPEC of firm $\mathrm{j}$.

Upper/lower dual constraints for day-ahead load demand of LSE in the MPEC of firm $j$.

Dual constraint for $P O P_{e v, j}$ of EV aggregator in the MPEC of firm $j$.

Dual constraint for $P_{g, j}^{D A}$ of CGenCos in the MPEC of firm j.

Dual constraint for $P_{w, j}^{D A}$ of WGenCos in the MPEC of firm $j$.

Dual constraint for $L_{d, j}^{D A}$ of LSE in the MPEC of firm j.

Dual constraints for of dual variable $\mu_{e v, t}^{D A^{\max }}, \mu_{e v, t}^{D A^{\min }}$ of EV aggregator in the MPEC of firm j.

Dual constraints for of dual variable $\mu_{g, t}^{D A^{\max }}, \mu_{g, t}^{D A^{\min }}$ of CGenCos in the MPEC of firm j.

Dual constraints for of dual variable $\mu_{w, t}^{D A^{\max }}, \mu_{w, t}^{D A^{\min }}$ of WGenCos in the MPEC of firm j.

Dual constraints for of dual variable $\mu_{d, t}^{D A^{\max }}, \mu_{d, t}^{D A^{\min }}$ of LSE in the MPEC of firm $\mathrm{j}$.

Upper/lower dual constraints for regulation up of EV aggregator power in the MPEC of firm $j$.

Upper dual constraint for real-time power of EV aggregator in the MPEC of firm $j$.

Upper/lower dual constraints for regulation down of EV aggregator power in the MPEC of firm $j$.

Lower dual constraint for real-time power of $\mathrm{EV}$ aggregator in the MPEC of firm $j$.

Upper/lower dual constraints for regulation up of CGenCos power in the MPEC of firm $j$. 


$$
\begin{aligned}
& Z_{g, s, j}^{u p} \\
& \text { Upper dual constraints for real-time power of CGenCos. } \\
& Z_{g, s, j}^{d_{\text {max }}}, Z_{g, s, j}^{d_{\text {min }}^{\text {min }}} \\
& Z_{g, s, j}^{\text {down }} \\
& Z_{w, s, j}^{c_{\max }}, Z_{w, s, j}^{c^{\min }} \\
& Z_{d, s, j}^{c^{\max }}, Z_{d, s, j}^{c^{\min }} \\
& Z_{e v, s, j}^{E E V^{\max }}, Z_{e v, s, j}^{E E V^{\min }} \\
& Z R_{e v, j}^{u p^{s}} \\
& Z R_{e v, j}^{d^{s}} \\
& Z R_{g, j}^{u p^{s}} \\
& Z R_{g, j}^{d^{s}} \\
& Z P_{w, j}^{C^{s}} \\
& Z L_{d, j}^{C^{s}} \\
& Z E_{e v, j}^{D A} \\
& X_{e v, s, j}^{u p^{\max }}, X_{e v, s, j}^{u p^{\min }} \\
& X_{e v, s, j}^{u p}, X_{e v, s, j}^{\text {down }} \\
& \text { Upper/lower dual constraints for regulation down of } \\
& \text { CGenCos power in the MPEC of firm } \mathrm{j} \text {. } \\
& \text { Lower dual constraints for real-time power of CGenCos } \\
& \text { in the MPEC of firm } j \text {. } \\
& \text { Upper/lower dual constraints for wind power } \\
& \text { curtailment of WGenCos in the MPEC of firm } j \text {. } \\
& \text { Upper/lower dual constraints for load demand } \\
& \text { curtailment of LSE in the MPEC of firm } j \text {. } \\
& \text { Upper/lower dual constraints for energy capacity of EV } \\
& \text { aggregator in the MPEC of firm } j \text {. } \\
& \text { Dual constraint for } R_{e v, j}^{u p^{s}} \text { of } \mathrm{EV} \text { aggregator in the } \\
& \text { MPEC of firm } j \text {. } \\
& \text { Dual constraint for } R_{e v, j}^{d^{s}} \text { of } \mathrm{EV} \text { aggregator in the } \\
& \text { MPEC of firm } j \text {. } \\
& \text { Dual constraint for } R_{g, j}^{u p^{s}} \text { of CGenCos in the MPEC of }
\end{aligned}
$$




$X_{e v, s, j}^{d^{\text {max }}}, X_{e v, s, j}^{d^{\text {min }}}$
$X_{g, s, j}^{u p^{\text {max }}}, X_{g, s, j}^{u p^{\text {min }}}$
$X_{g, s, j}^{u p}, X_{g, s, j}^{\text {down }}$
$X_{g, s, j}^{d^{\text {max }}}, X_{g, s, j}^{d^{\text {min }}}$
$X_{w, s, j}^{c^{\text {max }}}, X_{w, s, j}^{c^{\text {min }}}$
$X_{d, s, j}^{c^{\text {max }}}, X_{d, s, j}^{c^{\text {max }}}$
$\emptyset_{j}^{E V}$
$X_{e v, j}^{E E V^{\text {max }}}, X_{e v, j}^{E E V^{\text {min }}}$
$\oint_{j, s}^{R T}$
$\emptyset_{j}^{D A}$
$\delta_{j}^{D A}$

Dual constraints for of dual variable $\mu_{e v, s, j}^{d^{m a x}}, \mu_{e v, s, j}^{d^{\text {min }}}$ of EV aggregator in the MPEC of firm j.

Dual constraints for of dual variable $\mu_{g, s, j}^{u p^{\max }}, \mu_{g, s, j}^{u p^{\text {min }}}$ of EV aggregator in the MPEC of firm $\mathrm{j}$.

Dual constraints for of dual variable $\mu_{g, s, j}^{u p}, \mu_{g, s, j}^{\text {down }}$ of EV aggregator in the MPEC of firm $\mathrm{j}$.

Dual constraints for of dual variable $\mu_{g, s, j}^{d^{\text {max }}}, \mu_{g, s, j}^{d_{\text {min }}}$ of EV aggregator in the MPEC of firm $\mathrm{j}$.

Dual constraints for of dual variable $\mu_{w, s, j}^{c^{\max }}, \mu_{w, s, j}^{c^{\min }}$ of EV aggregator in the MPEC of firm $\mathrm{j}$.

Dual constraints for of dual variable $\mu_{d, s, j}^{c^{\max }}, \mu_{d, s, j}^{c^{\max }}$ of EV aggregator in the MPEC of firm $\mathrm{j}$.

Dual constraints for of dual variable $\mu_{e v, j}^{E E V^{\max }}, \mu_{e v, j}^{E E V^{\text {min }}}$ of EV aggregator in the MPEC of firm $\mathrm{j}$.

Dual constraint of day-ahead energy balance in the MPEC of firm $\mathrm{j}$.

Strong duality equality for the day-ahead lower-level problem in the MPEC of firm $j$.

Dual constraint of real-time energy balance in the MPEC of firm $\mathrm{j}$.

Strong duality equality for the real-time lower-level problem in the MPEC of firm $j$.

Dual constraint of EV energy balance in the MPEC of firm $\mathrm{j}$.

Strong duality equality for the EV lower-level problem in the MPEC of firm $j$. 


\section{List of Abbreviations and Acronyms}

$\begin{array}{ll}\text { ARMA } & \text { Auto Regressive Moving Average } \\ \text { ARIMA } & \text { Auto Regressive Integrated Moving Average } \\ \text { CGenCo } & \text { Conventional Generating Company. } \\ \text { DoD } & \text { Depth of Discharging } \\ \text { DA } & \text { Day-ahead. } \\ \text { DAM } & \text { Day-ahead Market. } \\ \text { EPEC } & \text { Equilibrium Problem with Equilibrium Constraints. } \\ \text { ESS } & \text { Energy Storage System. } \\ \text { EV } & \text { Electric Vehicle. } \\ \text { GenCo } & \text { Generating Company. } \\ \text { KKT } & \text { Karush-Kuhn-Tucker. } \\ \text { LSE } & \text { Load Side Entity. } \\ \text { MO } & \text { Market Operator. } \\ \text { MILP } & \text { Mixed-Integer Linear Programming } \\ \text { MPEC } & \text { Mathematical Program with Equilibrium Constraints. } \\ \text { POP } & \text { Preferred operating point. } \\ \text { SOC } & \text { State of Charge } \\ \text { V2G } & \text { Vehicle to Grid. } \\ \text { WGenCo } & \text { Wind Generating Company. } \\ \text { RT } & \text { Real-time. } \\ \text { RTM } & \text { Real-time Market. }\end{array}$




\section{Chapter 1}

\section{Introduction}

\subsection{Preface}

Electric power generators, transportation systems, and residential houses contribute $41 \%, 23 \%$, and $6 \%$ of the world greenhouse gases, respectively [1]. The strong dependence on foreign energy sources known as "oil addiction", the growing awareness of global warming impacts of $\mathrm{CO}_{2}$ emissions, and high energy efficiency are the driving forces for the increase in the penetration of renewable energy and electric vehicles $(\mathrm{EVs})[2,3]$.

According to the International Energy Agency (IEA), the Electric Vehicles Initiative (EVI) members, with about $63 \%$ of the world's total vehicle demand, plan to consider $83 \%$ of EV sales by 2020 [4]. The Global Wind Energy Council has reported that wind power could supply up to $17-19 \%$ and $25-30 \%$ by 2030 , and 2050 of the global electricity supply, respectively [5].

Therefore, power systems are likely to face increasing energy imbalance in both generation and load in the near future. Turning a load on/off or 
increasing/decreasing the demand can be effective to balance power in the grid. The coordinated control of the number of EVs (controllable loads) can potentially balance power in the grid [6].

A mechanism is necessary to integrate the electrified transportation within the power system and encourage EV owners as active players in the electricity market [7-8]. Unidirectional vehicle-to-grid (V2G) technology is a mechanism whereby the EV load aggregators in a sizeable number can participate in energy and ancillary services markets due to much faster ramping capability than gas turbines and a cheaper approach than energy storage systems [9-10].

EV aggregators as new market players have to compete with other market players while motivating the consumers to take part in the market. Moreover, the increasing penetration of $\mathrm{EVs}$ is another factor that contributes to energy imbalance [11].

Wind generating companies (WGenCos) participate in electricity markets despite their uncertainty to maximize the expected payoff, similarly to the other market producers with consideration of WGenCo as price-maker market players. Both wind generation uncertainty and energy price fluctuations are contributing factors to the decrease of the competitiveness of WGenCos in the energy market $[12]$

The main operational problem associated with a high wind penetration and EVs comes from intermittency and unpredictability. Also, EVs might impose excessive load on the grid [2]. Therefore, price and the market outcomes are influenced by the participation of the wind power and EV loads in high penetration [13].

This dissertation considers market analysis to investigate the influence of unidirectional V2G and wind power in the future smart grid. 


\subsection{Review of Current Trends in Integrating Electric Vehicles into Grids}

There have been several studies on the integration of EVs into power systems [1421]. In distribution systems, [22-26] evaluate the impact of EVs charging on the power quality such as voltage profile, harmonic and power losses. In [27-31], optimal scheduling for charging of EV is evaluated. Optimal siting and sizing of EV charging stations are considered in [32-34]. In transmission and generation systems, the benefits of V2G technology to a power system are investigated [35$38]$.

The first step to use the V2G technology is the economic aspect of EV aggregators. Several studies have also covered the economic aspects of integrating EVs into electricity markets [39-40]. Simulations have shown that EVs acting as smart storage can provide fast and accurate responses for frequency regulation and spinning reserves to aid in the integration of wind and solar power [10]. The definition of $\mathrm{V} 2 \mathrm{G}$ is the provision of energy and ancillary services to the electricity grid from EVs [41-43].

EV participation in the energy and ancillary service markets has been investigated in several studies. In [9], the EV participation considered bidirectional V2G interactions. Although V2G can be both unidirectional and bidirectional, the unidirectional $\mathrm{V} 2 \mathrm{G}$ is expected to be implemented first as it requires fewer infrastructures and reduces battery degradation by not requiring additional cycling for bidirectional power flow [8]. In [8-9], unidirectional smart charging with EV participation in the energy and regulation markets is studied without considering the stochastic nature of the process. 
Stochastic modelling of aggregated EVs and their impact on the optimal load profile of the power system is presented in [9]. However, discharging cost is considered constant and is not affected by depth of discharging (DoD) in these studies.

Currently EV usage is in its initial stage; when EV penetration becomes strong and influences the grid parameters, control of a large number of EVs to balance the entire power system will represent a challenge for power utilities. Several studies have demonstrated the benefits of coordination between wind power generators and EVs in power networks [44-49]. The authors of [50] have examined the effect of EV integration in a wind-thermal power system on emissions produced. In [51], a stochastic unit commitment model is used to simulate windthermal power system scheduling with different charging patterns for EVs to reduce the operating costs of a power system. However, these studies did not consider the intra-hour variability of the EV charging behaviour and wind energy generation which limit potential benefits of energy dispatch in the power system. In [52], coordinated wind-EV in three energy dispatching approaches, i.e., valley searching, interruptible and variable rate dispatching, is used without considering economic issues. Study [7] proposed coordinating unidirectional (V2G) services with energy trading. In [7], EV aggregators did not participate in the regulation market. In $[53,39]$, it has been shown that the highest benefits for EV owners are expected through participation in regulation markets.

There is a lack of a study on the impact of high wind penetration and EVs on the energy and balancing markets equilibria for the coordinated strategy in the literature. However, the limited works study the impact of wind power uncertainty on market equilibria [13]. In [54], an offering strategy for a wind power producer with market power that participates in the day-ahead market as a price-maker and in the balancing market as a deviator is proposed. 
Unlike [54], in [55], the producer is a price-taker in the day-ahead market, but a price-maker in the balancing market, and aims at optimizing its expected revenue from these market floors.

In [13], the equilibrium problem in a pool-based two-settlement electricity market is investigated where wind power is included in the generation portfolio of strategic producer in addition to its dispatchable units. Also, in [56], the bidding strategy of the EV aggregator is formulated as a bilevel problem to take into account the EV aggregator to potentially influence market prices without considering the impact of EV tariff and real-time market.

Therefore, in almost all previous studies, the EV aggregators have been considered as price-takers with exogenous formation of EV aggregator tariff. However, with high penetration of EV numbers, EV aggregators will have a more important role in the future electricity market [2].

\subsubsection{Common Modelling Assumptions}

The main modelling assumptions considered via this dissertation are described in this section.

\section{A. Stochastic Programming Problems}

Decision-making problems in the electricity market include imperfect (incomplete) information due to uncertainties in wind speed, energy prices, and the number of EVs [57]. In decision making under uncertainties, stochastic programming is used to make optimal decisions throughout multiple plausible scenarios of each stochastic variable. There are two sets of decision variables in two-stage stochastic programming problems including here-and-now (first stage) and waitand-see (second stage) decisions. The here-and-now decisions are made before the 
realization of the stochastic variables. The wait-and-see decisions are made based on the realized scenarios. Hence, the first stage decisions affect the second stage decisions $[7,57-58]$.

\section{B. Scenario Generation and Reduction Techniques}

Appropriate scenario generation and reduction methods are necessary to properly represent the stochastic programming problems. There are several different scenario generation and reduction techniques for stochastic programming [59]. The Monte Carlo simulations are applied to generate scenarios in [59]. In [60], time series models are used to generate scenarios for prices in electricity markets. The most common scenario-reduction technique is based on Kantorovich distance [61]. In [62], a scenario generation and reduction technique for price forecasting is based on the roulette wheel mechanism.

In this dissertation, scenario generation and reduction techniques are used for simulating wind speed, energy price, and the number of EVs engaged as follows.

\section{- Wind and Energy Price Scenarios}

Wind speed forecasting for the next day can be obtained from numerical meteorological programs, however, forecasts are never perfect. The Auto Regressive Moving Average (ARMA) model is used to simulate wind speed forecast errors [57, 63-65].

The estimation and adjustment of ARMA models have been investigated in literature. In this thesis, the first order of the ARMA model, ARMA (1,1), is used to simulate wind speed forecasting errors. 
Once a large number of scenarios are generated, the wind speed scenarios are transformed into power scenarios through the power conversion curve for each wind turbine $[12,65]$.

Similarly, Auto Regressive Integrated Moving Average (ARIMA) models have been applied to forecast electricity prices, which appear non-stationary when the processes present a periodic or seasonal pattern [57, 65-66]. The details of the methods are described in Appendix A.1.

\section{- EV Penetration Scenarios}

The EV availability at each interval has associated unplanned departure and arrival probabilities. The number of EVs is considered to be random, and Monte Carlo simulations are used to generate possible scenarios $[9,35,66]$. The details of the methods are described in Appendix A.2.

\section{- Scenario Reduction}

In stochastic optimization problems with various inherent uncertainties, a large number of scenarios can emerge. It can, therefore, be computationally expensive. Therefore, a technique for reducing the number of scenarios is required.

In this dissertation, the scenario reduction algorithm is based on $[67,68]$. The basic idea of the scenario reduction is to eliminate scenarios with lowprobabilities, and cluster similar scenarios $[12,67,68]$. The new probability of a preserved scenario is determined as the sum of its initial probability and the probabilities of similar scenarios that have been eliminated. We used SCENRED as a tool for scenario reduction [69]. The details of these methods are described in Appendix A.3. 


\section{Competition Modelling of the Electricity Market}

The electricity market is categorized into two kinds of competition comprising perfect and imperfect competitions [70-71]. In the perfect completion model, the participants are price-takers and their decisions do not affect the market price because of their small potential output. In the imperfect completion model, some strategic players (participants) affect the market price through their decisions. The electricity market most commonly includes a few strategic players as price makers and some (a number of) participants as price takers. Imperfect competition can be modelled using the Cournot model, the Bertrand model, or the Supply Function model.

1. The Bertrand model: In this model, competition among players is in prices [72]. A player offers a price to maximize its profit and the market decides the production quantity $[73$.

2. The Cournot model: In this model, competition among players is in quantity [72]. A player maximizes its profit by an optimal quantity in the Cournot model, and the other player's quantity is considered to not be changed $[73,77-82]$.

3. The Supply Function model: this model is an extension of both the Bertrand and Cournot models [72]. Each participant submits the supply function offer to the electricity market including quantity and price offers. The Supply Function model is suitable for the pool-based market compared with Cournot, and Bertrand [72,83-85].

In [86], Supply Function models have been applied in the British Electricity Spot Market. 
In [87], pure strategy Nash equilibria have been developed when GenCos participate through a supply function by formulating as a mixed-integer linear program (MILP) that does not require any discretising approximations.

In [88], a stepwise supply function model has been used in which a producer submits a set of stepwise price-quantity offers to the market operator (MO).

In [89], the impacts of large-scale integration of intermittent resources (such as wind energy) on electricity market prices using a supply function equilibrium (SFE) model have been studied.

In this dissertation, an SFE model is used in which Conventional Generating Companies (CGenCos), WGenCos, and EV aggregators submit supplyoffers/demand-bids to the MO [88].

\subsection{Mathematical Structures of Electricity Markets}

The combination of regulated (old) and deregulated (new) electricity markets with either perfect or imperfect competition leads to complementarity models. These problem comprise of multiple optimization problems in which primal (power quantities) and dual variables (prices) are constrained together [71].

The mathematical structures of the electricity market can be categorized into single and multiple optimization problems [71].

In a single optimization problem, the WGenCo and EV aggregator as pricetakers aim to maximize their objective function associated with equality or inequality constraints as explained in Chapter 3. Fig. 1.1 illustrates the structure of an optimization problem specifying its components. 


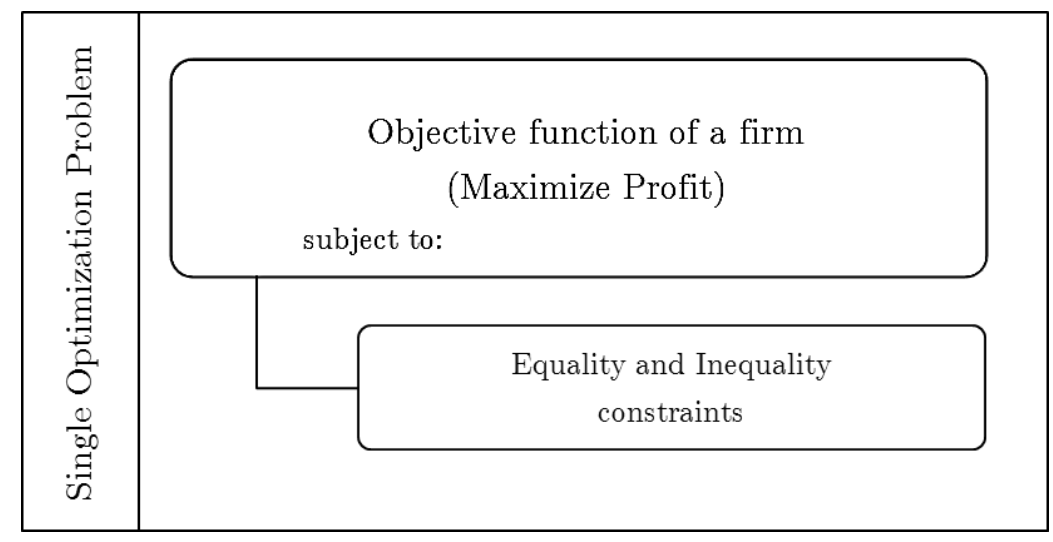

Fig. 1.1 Optimization problem

In multiple optimization problems, the strategic firms such as WGenCos, EV aggregators, and other players as price-maker submit supply-offers/demand-bids to the MO to participate in the electricity market.

A bilevel (hierarchical) model is used in this dissertation to model the behaviour of each player [90-94]. A bilevel problem includes an upper-level problem and a set of lower-level problems which are limited by the upper and lower equality and inequality constraints. The bilevel model can be formulated as a single-level stochastic mathematical program with equilibrium constraints (MPEC) [96]. The lower-level problems are continuous linear and they can be replaced by their Karush-Kuhn-Tucker conditions (KKTs) as shown in Fig. 1.2 $[91]$.

As explained in Chapters 4 and 5, the firm's profit maximization is developed as an upper-level problem, and lower-levels problems represent the market clearing. The MPEC problem is converted into an MILP problem using branchand-cut algorithms [93].

Multiple MPEC problems constitute an equilibrium problem with equilibrium constraints (EPEC) which are solved through the solutions associated with the strong stationarity conditions (KKTs) of all MPECs as illustrated in Fig. 1.3 [91]. 
1.3 Mathematical Structure of Electricity Markets

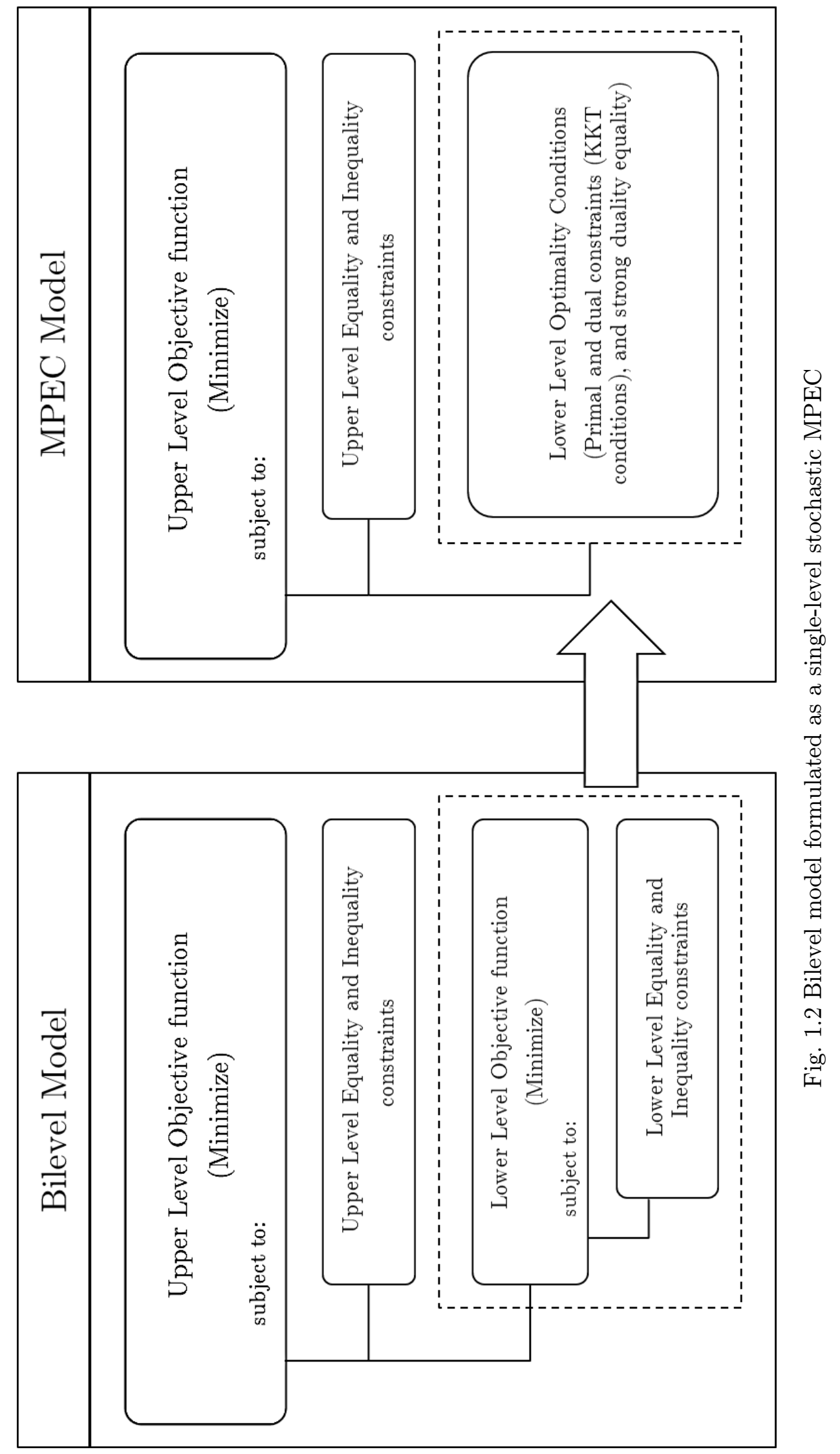




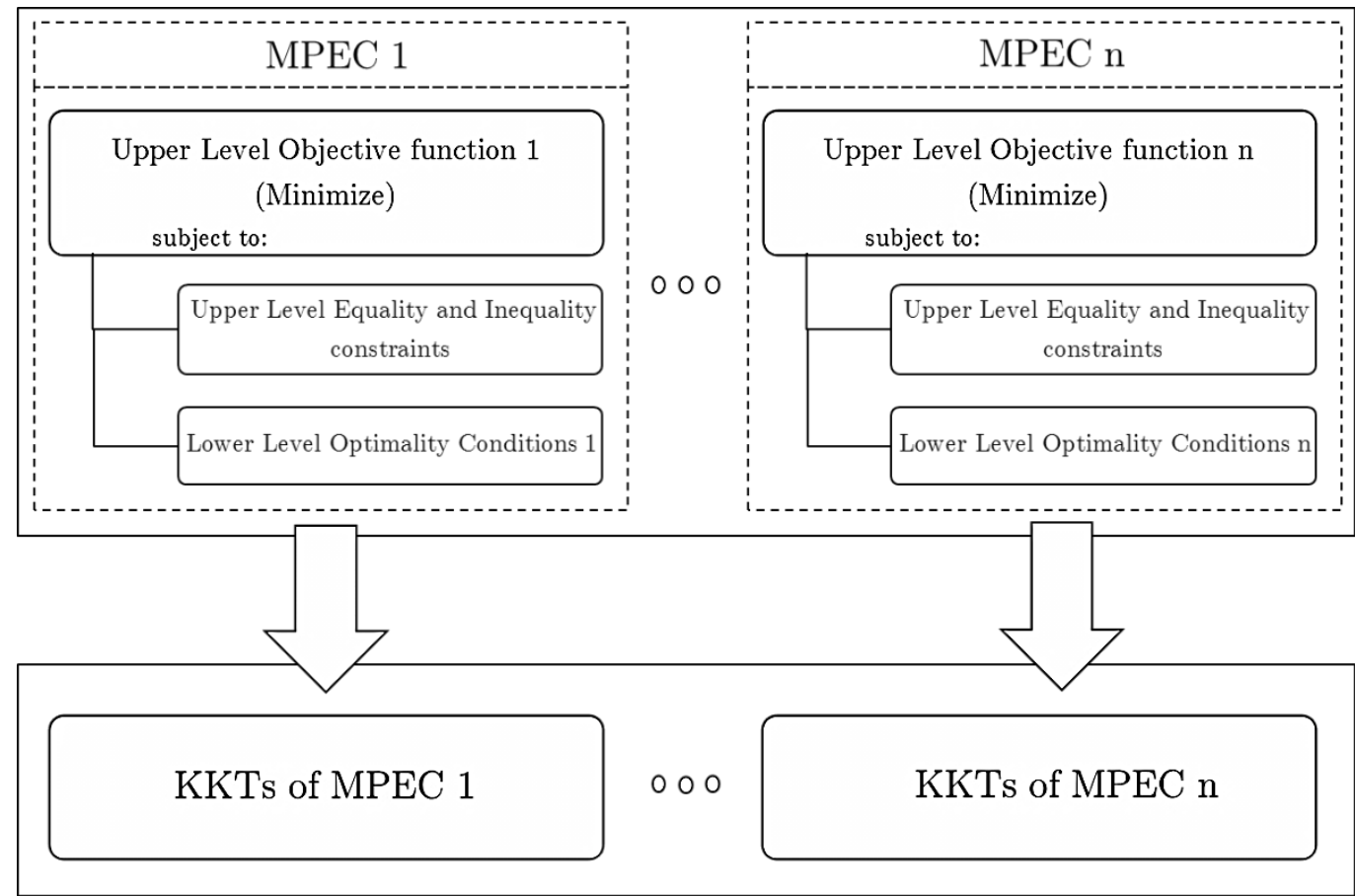

Fig. 1.3 An Equilibrium Problem with Equilibrium Constraints (EPEC)

\section{$1.4 \quad$ Project Objectives}

This thesis focuses on revaluating the electricity market associated with the new players such as the EV load aggregator, and analysing the impact of coordination strategy on the market outcomes and prices. The coordination occurs between the EV load aggregator and generating companies through V2G technology.

In this document, we investigate the two studies as follows:

- Development of a power exchange between the EV load aggregators and WGenCos considered as price-takers in the energy and ancillary service markets.

- Development of a power exchange between the EV load aggregators and all generating companies considered as price-makers in the single and multiple firms. 


\subsection{Thesis Organization}

The dissertation's organization is as follows:

Chapter 1 provides an introduction consisting of preface, the literature review, the modelling assumptions, the mathematical structures of electricity markets, and the thesis organization and objectives.

Chapter 2 provides a review of EVs in electricity markets. Firstly, divers electricity markets and their time framework are explained. The role of demand dispatch in regulation ancillary services is explained. Finally, the role of the EV aggregator in regulation ancillary service and energy markets and their mathematical formulation are represented.

Chapter 3 presents an approach to energy exchange between electric vehicle $(\mathrm{EV})$ load and wind generation utilities participating in the day-ahead energy, balancing, and regulation markets. An optimal bidding/offering strategy model is developed to mitigate wind energy and EV imbalance threats, and optimize EV charging profiles. A new strategy model is based on optimizing decision making of a WGenCo in selecting the best option among the use of the balancing or regulation services, the use of the energy storage system (ESS) and the use of all of them to compensate wind power deviation. Energy imbalance is discussed using conventional systems, ESS, and EV-Wind coordination; results are compared and analysed. Stochastic intra-hour optimization is solved by MILP. Uncertainties associated with wind forecasting, energy price, and behavior of EV owners based on their driving patterns, are considered in the proposed stochastic method, and validated through several case studies. 
Chapter 4 proposes an approach to investigate the impact of merging EV load aggregators into the portfolio of a strategic firm, and the influence of this strategy on electricity market equilibrium and EV tariff for the sake of increasing a firm's profit while retaining social welfare and optimizing EV charging profiles. A strategic firm trades electric energy in an electricity pool including the dayahead (DA) and real-time (RT) markets at wholesale-level while EV owners connect to an EV aggregator in order to take part in the market at EV-level. A stochastic intra-hour bilevel model is developed, which includes a) the firm's profit maximization as an upper-level problem and b) DA and RT social welfare, and the EV owner battery energy maximizations with the corresponding endogenous price formation as the lower-level problems. The problem is formulated from an MPEC to a MILP. Uncertainties of wind speed, and behavior of EV owners based on their driving patterns are considered in the proposed strategy, and validated through several case studies.

Chapter 5 proposes a methodology to describe market equilibria in a poolbased electricity market, where each CGenCo, WGenCo and EV aggregator is defined in multiple strategic firms. A bilevel model for each strategic firm is developed including expected payoff as upper-level problem, and several lowerlevel problems represent the market clearing. Each bilevel model is converted into a single-level MPEC by replacing the lower-level problems with their primal-dual optimality conditions. An EPEC is formulated to consider all single firms' MPECs. The optimality conditions of the EPEC are derived by replacing each MPEC with its KKT conditions. The optimality conditions of the EPEC are linearized by formulating and solving an MILP problem. A case study is discussed to validate the proposed model to recognize meaningful market equilibria, where the strategic CGenCo, WGenCo and EV aggregator participate in energy and 


\subsection{Thesis Organization}

ancillary services. We compare different cases including coordination and incoordination strategies, with and without EVs, the impact of different EV numbers, and different combination of units in multiple firms.

Chapter 6 concludes this thesis, providing the major contributions of the thesis and suggests some directions for future work targeting to develop the research studies presented here.

Appendix A provides mathematical background and details regarding the scenario generation and reduction techniques for simulating wind speed, energy price, and the number of EVs engaged.

Appendix B provides the mathematical background of formulating absolute values of variables as a MILP.

Appendix C contains additional data from Chapter 5 which are not directly relevant to the discussions of Chapter 5, but they represent additional details and complements. 



\section{Chapter 2}

\section{Electric Vehicles in Electricity Markets}

\subsection{Introduction}

This chapter provides an overview of EVs in electricity markets. The concept of V2G and the role of EV aggregators in regulation ancillary service and energy markets and their mathematical formulation are represented.

The rest of the chapter is organized as follows. Section 2.2 provides a general description of the different electricity markets and their time framework, and the roles of the market players. Section 2.3 provides some insight into the uncertainty in power systems. Section 2.4 explains the demand dispatch in regulation ancillary services. Section 2.5 explains further the concept of unidirectional and bidirectional V2G and EV aggregators. Finally, Sections 2.6 and 2.7 present the regulation ancillary services via unidirectional V2G and their mathematical formulation in electricity market. 


\subsection{Electricity Markets}

Electricity markets are multi-commodity markets. The different energies (products) and services are traded in the different power marketplaces. The products include the base energy, reserves, regulation, and balancing energy which are altered in control method, response time, duration of the power dispatch, contract terms, and price [71]. These energy and services are traded in the marketplaces such as pool, reserve and regulation, bilateral, and future markets [39]. In this dissertation, the participants in the electricity markets are load-side entities (LSE), CGenCos, load aggregators, and WGenCos described as follows.

1. The LSE: provide the electrical demand and energy requirements to the enduse customers that cannot participate directly in the electricity markets.

2. CGenCos: supply and sell the electricity energy generated by fossil fuels or nuclear energy in the electricity market. Dispatchable CGenCos (such as natural gas and coal plants) may participate in the reserve and regulation markets to provide reserve power and load following capacity, respectively.

3. Load Aggregator: purchases the electricity to supply energy to sizable special clients (such as EV owners) through bilateral contracts in the futures market. The clients connect to the load aggregator in order to take part in the market indirectly. The Load aggregator determines its energy capacity and tariff for customers based on achieved market data.

4. WGenCos: are non-dispatchable producers generating electricity energy by wind power. A WGenCo must participate in the balancing market to compensate its deviations from the scheduled generation because of inherent intermittency of wind energy. 


\subsubsection{Pool-based Electricity Market}

A pool-based electricity market typically includes the day-ahead market and realtime (balancing) market trading on a short-term basis [57].

In the day-ahead market, producers and consumers submit respectively supplyoffers and demand-bids to the MO to participate in the electricity market. The MO runs the day-ahead market clearing process to determine day-ahead price and energy quantities schedules [65].

In the real-time market, producers and consumers submit balancing offers to the MO to participate in the electricity market. The balancing (real-time) market is cleared based on achieved day-ahead market data to balance the excess and deficit of production and consumption. Fig. 2.1 shows the structure of a poolbased electricity market [57].

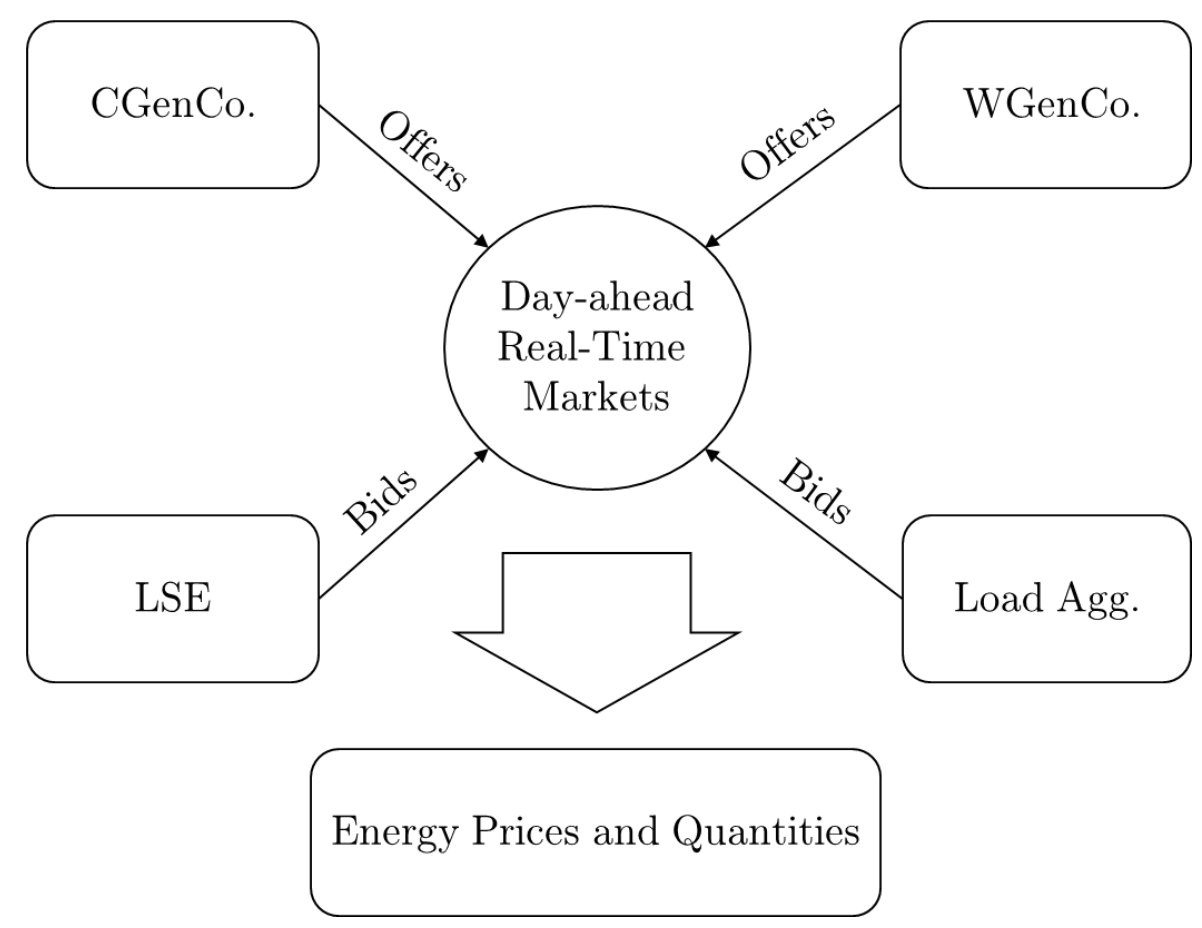

Fig. 2.1 The structure of a pool-based electricity market 
The time framework for the day-ahead market is the whole day (d), which is cleared at $10 \mathrm{AM}$ of the day (d-1). The balancing market ensures the real-time balance between generation and demand by offsetting the difference between the real-time operation and the last energy program cleared in the market. For this reason, this market remains open until 10 minutes before the delivery hour [65].

\subsubsection{Reserve and Regulation Markets}

The reserve is an important product to ensure the secure system operation and energy delivery in the huge fluctuations of the intermittent energy generation and demand. The reserve market supplies standby power (spinning and non-spinning) which is cleared in the day-ahead market $[39,57]$.

The regulation market provides up and down real-time regulation capacities to match generation and load demand (load-following). The amount of regulation capacity contracted is the total amount by which power can deviate from a baseline level $[39,57]$. The baseline is often called the preferred operating point (POP) [6]. It represents the average level of operation for a market participant providing regulation services [9].

Participants submit supply-offers for regulation capacity (or POP) to the market operator which is cleared in the day-ahead market. In the real time market, the power generation capacities might be increased or decreased from a baseline level called "regulation up" and "regulation down", respectively [53].

The regulation up and down are never provided at the same time, although a producer can contract to provide one of them or both over the same contract period [57]. 


\subsubsection{The Futures Market and Bilateral Contracts}

The futures market is an auction market in which participants buy and sell physical or financial products to trade on a medium- or long-term horizon [57].

A bilateral contract is a free agreement arranged outside an organized marketplace between a generating company (GenCo) and a load aggregator, or a load aggregator and some customer (such as EVs) shown in Fig. 2.2.

Fig. 2.3 shows the clearing times of future, day-ahead, reserve, regulation, and balancing (real-time) markets.

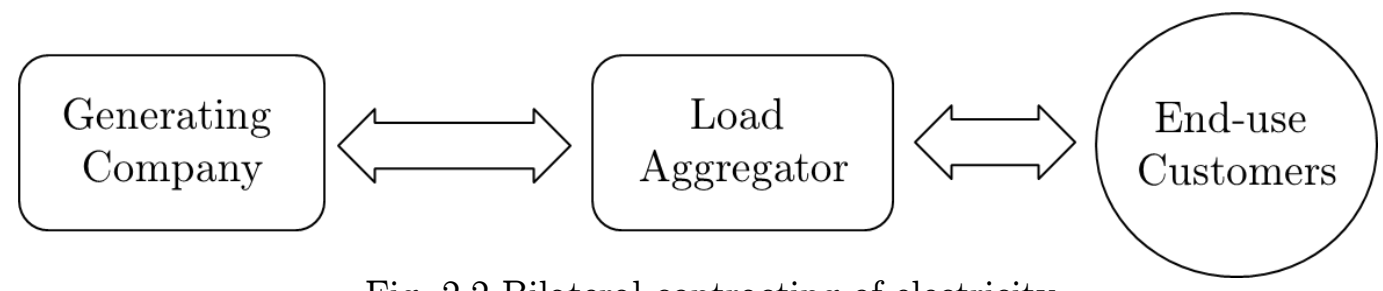

Fig. 2.2 Bilateral contracting of electricity

\subsection{Uncertainty in the Power Grid}

To maintain network frequency as close to the nominal value (50 or $60 \mathrm{~Hz}$ ) as possible, a real-time balance of generation and load have to be operated. Any deviation between load and generation is compensated by adding or subtracting power. In other words, generation is controlled to follow load [39].

With high penetration of renewable generation such as solar and wind energy, the load-following strategy faces new challenges. Wind and solar energy generation are highly unpredictable and quickly changeable. The more conventional generation capacities (such as coal and natural gas plants) are required to provide the ancillary services and fast ramping for the load-following with increases in intermittent renewable energy sources [6]. 


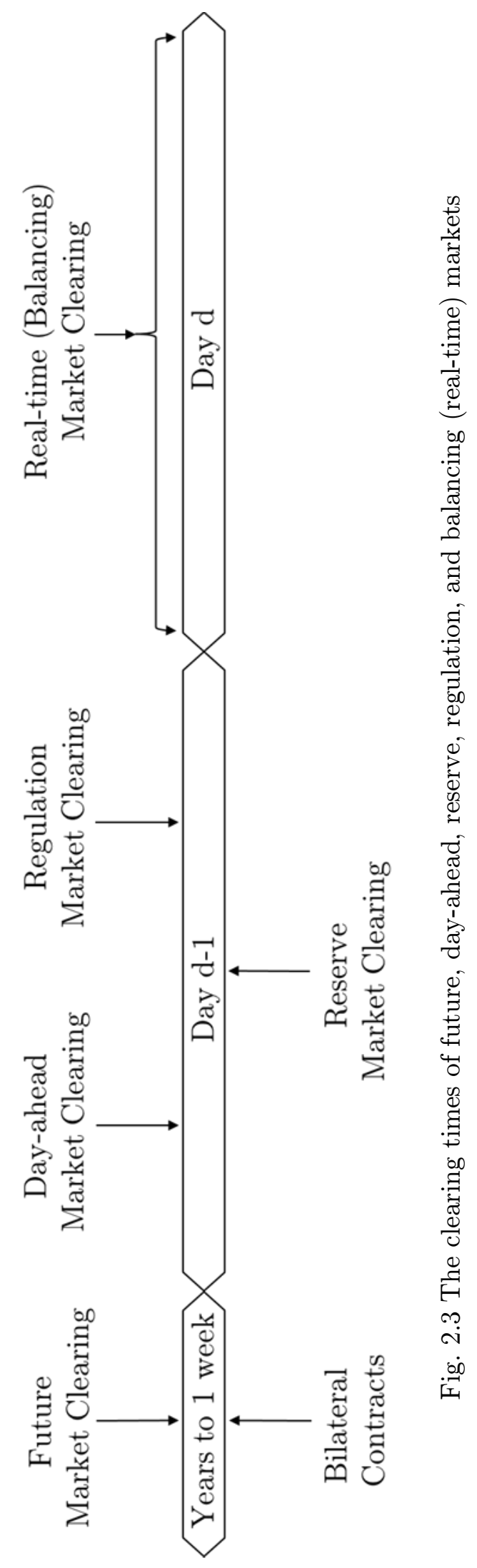


A approach is introduced as a direct control of some loads [6]. Turning a load on/off or increasing/decreasing the demand can be effective to balance power in the grid. Therefore, without adding new dispatchable generation capacity, we can compensate the imbalanced energy in the network via the dispatchable loads under demand dispatch [9].

\subsection{Demand Dispatch}

Demand dispatch (DD) is, in some sense, similar to demand response (DR) with the main difference that DR is used only to shed loads at peak times, while DD is intended to be used actively at all times [6]. Demand dispatch is a generalization of the term DR.

The application of DD could provide regulation ancillary services to balance load and generation on the grid in the different time scale from hours to seconds. However, conventional generators can ramp up and down for load-following strategy. Conventional generators respond slowly and need to deal with higher emissions, lower efficiency, and limited ramp rates. Energy storage such as flywheel and batteries could be used for regulation ancillary services, but they cause high cost and complexity on the power system $[6,43,53]$.

Some kinds of load called dispatchable loads turn on/off repeatedly and become generation-following under DD. Turning some loads on/off such as lights would cause discomfort for the electricity consumer. However, remotely controlled loads are unnoticeable such as electric hot water heaters, dishwashers, washers, dryers and charging an electric vehicle at night.

The criteria of a dispatchable load are [6]:

1) predictable to be forecasted for next day,

2) switchable to turn on/off frequently, 
3) flexible to draw power in the specific time and energy amount. According to the criteria mentioned above, one of the best candidates for demand dispatch would be electric vehicle loads through unidirectional V2G technology $[8,41]$.

\subsection{Vehicle-to-Grid (V2G)}

The concept of V2G is that EVs connect to the grid while parked. V2G application can be both unidirectional and bidirectional. In bidirectional V2G, EVs charge from the grid during low load periods and discharge to the grid when power is low [43]. In unidirectional $\mathrm{V} 2 \mathrm{G}$, the $\mathrm{EV}$ battery is considered as a dispatchable load to balance energy in the grid. V2G is expected to be implemented first as it requires less infrastructure and reduces the battery degradation by not requiring additional cycling for bidirectional power flow [9].

$\mathrm{V} 2 \mathrm{G}$ requires a connection system to the grid, communication system, and control and metering systems. The control signals can be transmitted through a cell phone and Internet networks [39].

The battery of an individual EV is too small to affect the grid. An EV aggregator including large numbers of EVs acts as an intermediary between individual EVs and the MO. EVs can connect to a third-party aggregator individually or as a fleet operator (a parking lot) within a city or whole area [43]. An EV aggregator can decrease the volume of communication signals to the MO. Therefore, the EV aggregators can reduce the market operator complexity and improve the cyber-security [11]. 


\subsection{Regulation Ancillary Services via Unidirectional V2G}

The EVs, when aggregated in a sizeable number, can play an important role in regulation service due to much faster ramping capability than gas turbines through V2G technology $[6,41,42]$.

The EV aggregator participates as dispatchable load in the energy and ancillary service markets by submitting energy bids and regulation offers. POP represents the average level of operation for a market participant providing regulation services. It is assumed that the EV aggregator can deviate from the day-ahead power-drawn (or POP) to amend imbalance energy by reducing or increasing their charging rate with consideration of EV aggregator energy constraints $[6,53]$.

Fig. 2.4 illustrates EV aggregator regulating while POP value is determined for one hour in the day-ahead market and regulation-up or -down dispatch deviates from POP following generation in the real-time market. Regulation-down and regulation-up dispatches represent increasing and decreasing the EV load demand, respectively.

\subsection{Mathematical Formulation of Unidirectional V2G in the Electricity Market}

A stochastic objective function (2.1) is to maximize the profits of the EV aggregator. According to the method discussed above, the EV aggregator's revenue is obtained by selling ancillary services, as well as selling energy to its clients at the tariff $\left(\rho^{T}\right)$. The aggregator encourages EV owners to join in by offering an attractive price for charging in comparison with petrol and energy prices. 


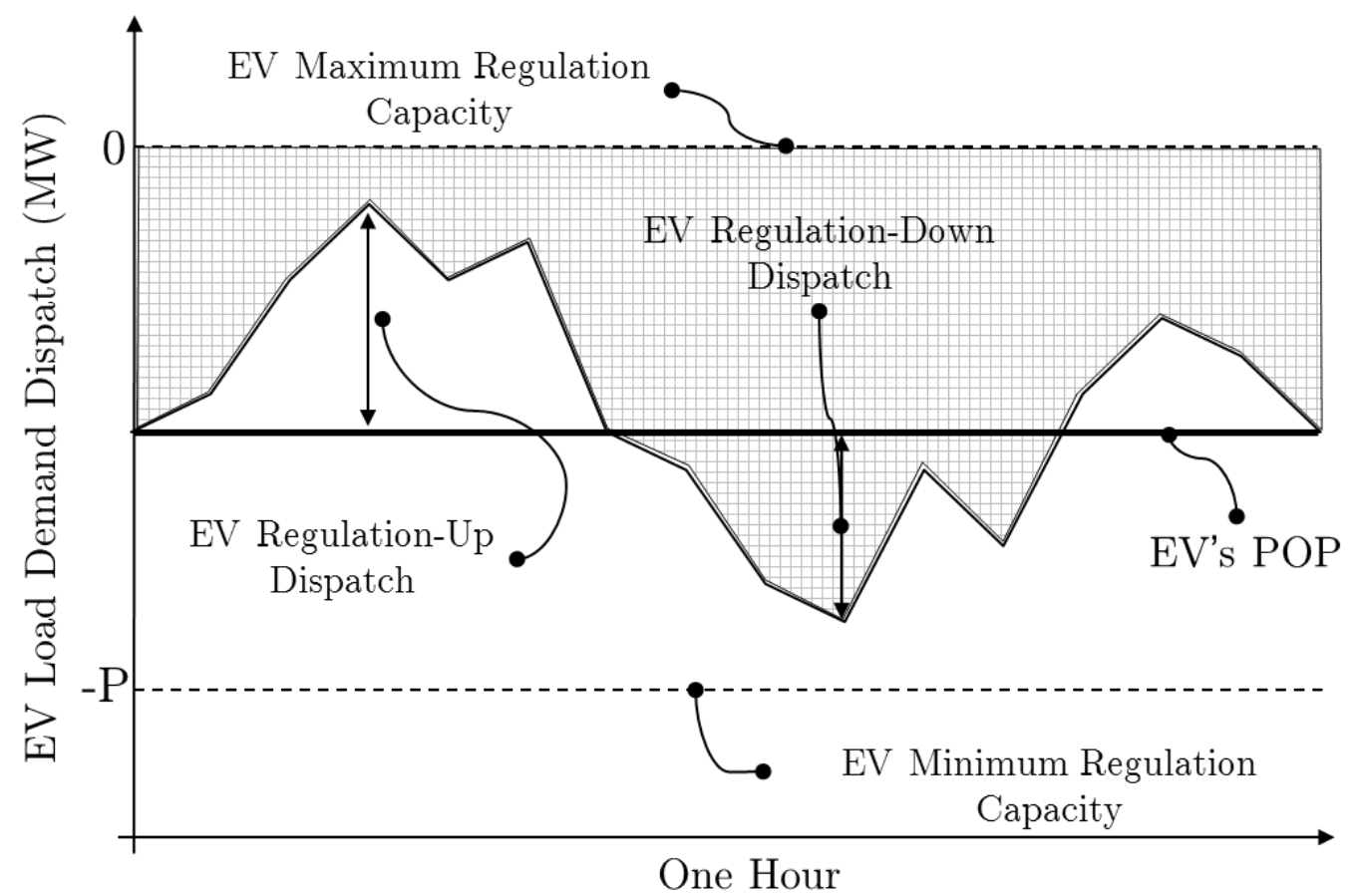

Fig. 2.4 EV aggregator regulating; the shaded area highlights the energy drawn from the grid

The EV aggregator's cost is associated with buying energy at the day-ahead market price $\left(\rho_{t}^{D A}\right)$ for regulation capacity (or POP). In this strategy, the power deviation between day-ahead and real-time markets is compensated by regulating the charging power down $\left(R_{e v, t, i}^{d o w n^{s}}\right)$ or up $\left(R_{e v, t, i}^{u p^{s}}\right)$ of POP at the real-time market price $\left(\rho_{s, t, i}^{R T}\right)$. Hence, the EV aggregator's payoff is represented as:

$$
\begin{aligned}
& \operatorname{Max}\left\{\left[\sum_{t=1}^{N_{T}} \sum_{e v=1}^{N_{e v}} \rho^{T}-\rho_{t}^{D A} P_{o} P_{e v, t}\right]\right. \\
& \left.+\pi_{s} \cdot\left[\frac{1}{N_{I}} \sum_{t=1}^{N_{T}} \sum_{e v=1}^{N_{e v}} \sum_{i=1}^{N_{I}}\left[\rho_{s, t, i}^{R T}-\rho^{T}\right]\left(R_{e v, t, i}^{u p^{s}}-R_{e v, t, i}^{d o w n^{s}}\right)\right]\right\}
\end{aligned}
$$


Constraints for the EV's POP, capacity to increase the charging rate for regulation down $\left(R_{e v, t, i}^{d o w n}\right)$, capacity to decrease the charging rate for regulation up $\left(R_{e v, t, i}^{u p^{s}}\right)$ are given in (2.2)-(2.6).

$$
\begin{aligned}
& P_{o P_{e v, t}} \leq P_{e v, c h}^{\max } \cdot N_{e v, s, t, i} \\
& P_{O} P_{e v, t}+R_{e v, t, i}^{d o w n^{s}} \leq P_{e v, c h}^{\max } \cdot N_{e v, s, t, i} \\
& R_{e v, t, i}^{u p^{s}} \leq P_{O} P_{e v, t} \\
& R_{e v, t, i}^{d o w n^{s}} \leq P_{e v, c h}^{\max } \cdot N_{e v, s, t, i} \cdot I_{e v, t, i}^{d o w n^{s}} \\
& R_{e v, t, i}^{u p^{s}} \leq P_{e v, c h}^{\max } \cdot N_{E V, s, t, i} I_{e v, t, i}^{u p^{s}}
\end{aligned}
$$

Since the regulation up and down are never provided at the same time, the status of regulation down or up is determined in (2.7).

$$
I_{e v, t, i}^{d o w n^{s}}+I_{e v, t, i}^{u p^{s}} \leq 1
$$

The energy balance equation for the EV aggregator is given in (2.8). The EV energy capacity in each intra-hour $\left(E_{e v, t, i}^{s}\right)$ is the EV energy capacity in prior intra-hour $\left(E_{e v, t, i-1}^{s}\right)$ plus energy charged by drawing power from the grid $\left(P_{e v, s, t, i}^{c h} . \Delta t\right)$ minus energy consumed by EVs while driving. The regulation capacity of the EV aggregator increases when the numbers of charging EVs increase and, vice versa as given in (2.8).

$$
E_{e v, t, i}^{s}=E_{e v, t, i-1}^{s}+P_{e v, s, t, i}^{c h} \cdot \Delta t-\left(1-N_{e v, s, t, i}\right) D_{e v, s, t, i}
$$

The energy capacity of EV aggregator is constrained in (2.9). 


$$
\begin{aligned}
E_{e v, t}^{\min } \cdot N_{e v, s, t, i} \cdot S O C_{e v}^{\min } \leq E_{e v, s, t, i} & \\
& \leq E_{e v, t}^{\max } \cdot N_{e v, s, t, i} S O C_{e v}^{\max }
\end{aligned}
$$

The constraint (2.10) imposes limits at the beginning and at the end of each interval of the energy capacity of the EV aggregator.

$$
E_{e v, t, i 0}^{s}=E_{e v, t-1, N I}^{s}
$$

The constraint (2.11) specifies the level of State of Charge (SOC) to be reached by time $(\mathrm{T})$ for a specified $\mathrm{EV}$ client. This constraint is an option for clients to set up the desirable SOC for their EVs at the time of expected commuting $(\mathrm{T})$.

$$
E_{e v, T, i}^{s}=E_{e v, t}^{\max } N_{e v, s, t, i} S O C_{e v}^{\max }
$$




\section{Chapter 3}

\section{Energy Exchange between Electric Vehicle Load and Wind Generating Utilities}

\subsection{Introduction}

An electricity market, e.g. PJM, may have a two-settlement system consisting of two markets: a day-ahead market (DAM) and a real-time balancing market. Generators are paid for any generation that exceeds their day-ahead scheduled quantities and are penalized for generation deviations below their scheduled quantities [6]. Whenever the scheduled day-ahead wind power generation deviates from the real-time market (RTM), the profitability of WGenCos decreases due to imbalance energy charges for the wind units $[6,35]$. 
To mitigate potential wind energy imbalance charges for WGenCos, the authors in [35], suggested a coordinated scheduling of wind energy units and storage units. However, the study was based on pumped storage power systems which represent only around $2.2 \%$ of the total generation with efficiency at about 75\%; they have high installation costs, and are limited to specific locations [10]. Although stored energy increases the economic value of wind energy [8], the use of large scale battery-based ESS is currently still prohibitively expensive.

The imbalanced energy exchange based on a dedicated coordination between EV load aggregators and WGenCos can potentially increase the competitiveness of WGenCos and EV-load customers in the energy market.

This chapter develops an optimal bidding/offering strategy for EV load demands in coordination with a WGenCo, thereby maximizing the WGenCo's competitiveness, optimizing EV charging profiles and mitigating imbalance energy provided by the balancing market. The EV aggregator participates in the energy and ancillary service markets while the WGenCo participates in the day-ahead energy and balancing markets.

The main contributions of the chapter are as follows [97-98]:

- The development of a two-stage stochastic linear programming (SLP)based optimal offering/bidding strategy model for the coordinated EVWind units participating in the day-ahead energy, balancing, and regulation markets.

- The development of an SLP-based optimal offering strategy model for the ESS-Wind units participating in the day-ahead energy, and balancing markets. 
- A new strategy model based on optimal decision making for selecting between the balancing, regulation services, and/or using ESS for a WGenCo to compensate wind power deviation.

- Comprehensive comparisons of three different cases comprising conventional systems (WGenCo without energy storage), WGenCo with ESS, and a coordinated EV-Wind energy exchange for dealing with energy imbalance.

- Consideration of the uncertainties associated with wind forecast, energy price, and EV owners' behaviour based on driving patterns.

The rest of this chapter is organized as follows. Section 3.2 discusses the market framework for the sake of conventional systems (WGenCo without storage), WGenCo with the energy storage system, and a power system with a coordinated EV-Wind energy exchange. Section 3.3 presents the mathematical model formulations of proposed models. Several case studies are discussed in Section 3.4. Finally, Section 3.5 concludes, summarizing the chapter.

\subsection{Market Framework}

The day-ahead market and a real-time balancing market are the two settlement systems considered in this chapter. Participants in the DAM submit supplyoffers/demand-bids to the system operator. These participants also submit supply-offers for the regulation capacity, and they may later submit revised regulation quantities, which are different from day-ahead offer quantities, without any penalty imposed [53].

Wind generation and EV load aggregators participate as price takers in the DAM by hourly offering/bidding amounts that are based on the day-ahead 
forecast while energy and price variations occur within minutes (i.e. intra-hour) $[35]$.

In this chapter, three different strategies are considered to deal with the energy imbalance for a WGenCo participating in short-term electricity markets (DAM and balancing). In the subsections below, these strategies are demonstrated using conventional systems (WGenCo without storage), WGenCo with the energy storage system, and a power system with a coordinated EV-Wind energy exchange.

\subsubsection{Conventional Systems}

WGenCos participate in the DAM and balancing market. The imbalance charge is imposed on the WGenCo to balance energy in the power system due to deviation of the RTM $[12,35]$. The WGenCo's payoff in this method is as follows:

$$
\begin{gathered}
\rho_{t}^{D A} P_{w, t}^{D A}-\rho^{P} \quad P_{w, t}^{D A}-P_{w, t, i}^{R T^{s}}-\rho_{s, t, i}^{R T}\left(P_{w, t}^{D A}\right. \\
\left.-P_{w, t, i}^{R T^{s}}\right)
\end{gathered}
$$

According to (3.2), the wind energy deviation between day- ahead and realtime is considered as the energy imbalance:

$$
\Delta P_{s, t, i}^{w}=P_{w, t}^{D A}-P_{w, t, i}^{R T^{s}}=P_{s, t, i}^{i m}
$$

\subsubsection{WGenCo with the Energy Storage System}

It is assumed that battery storage belongs to the WGenCo participating in the DAM and balancing market. The authors in [12] and [35] proposed a scheduling strategy for the coordination of wind and storage units without any flexibility for the WGenCo to adapt when the storage units fail. 
This section proposes a new scheduling strategy, which considers optimal decision making for WGenCos in selecting between the balancing market and ESS to compensate for wind power deviations. The WGenCo can decide whether to use the ESS or not based on penalties, energy prices, maintenance requirements and other factors. According to (3.3), the wind energy deviation between the DAM and RTM can be compensated by the battery storage system and balancing market. The optimization determines the one which is the most efficient.

$$
\Delta P_{s, t, i}^{w}=P_{w, t}^{D A}-P_{w, t, i}^{R T^{s}}=\left(P_{b, s, t, i}^{d c}-P_{b, s, t, i}^{c h}\right)+P_{s, t, i}^{i m}
$$

A degradation cost from the battery bank charging/discharging is considered in this method. The WGenCo's payoff is as follows:

$$
\rho_{t}^{D A} P_{w, t}^{D A}-\rho^{P} P_{s, t, i}^{i m}-\rho_{s, t, i}^{R T}\left(P_{s, t, i}^{i m}\right)-C_{b, s, t, i}^{d c}
$$

\subsubsection{Coordinated EV-Wind Energy Exchange}

In the method discussed above, the WGenCo participates in the short-term electricity market. The EV aggregator participates as dispatchable load in the energy and ancillary service markets by submitting energy bids and regulation offers. The amount of regulation contracted is the total amount by which power can deviate from a baseline level. The baseline is often called the POP (Preferred operating point) [6]. The term POP itself comes from ancillary services markets. It represents the average level of operation for a market participant providing regulation services [8]. It is assumed that the EV aggregator can deviate from the day-ahead power-drawn (or POP) to amend wind imbalance energy by reducing or increasing their charging rate with consideration of EV aggregator energy 
constraints. Therefore, the offer price in the day-ahead market does not change in the real-time market while offer quantities can be revised [17].

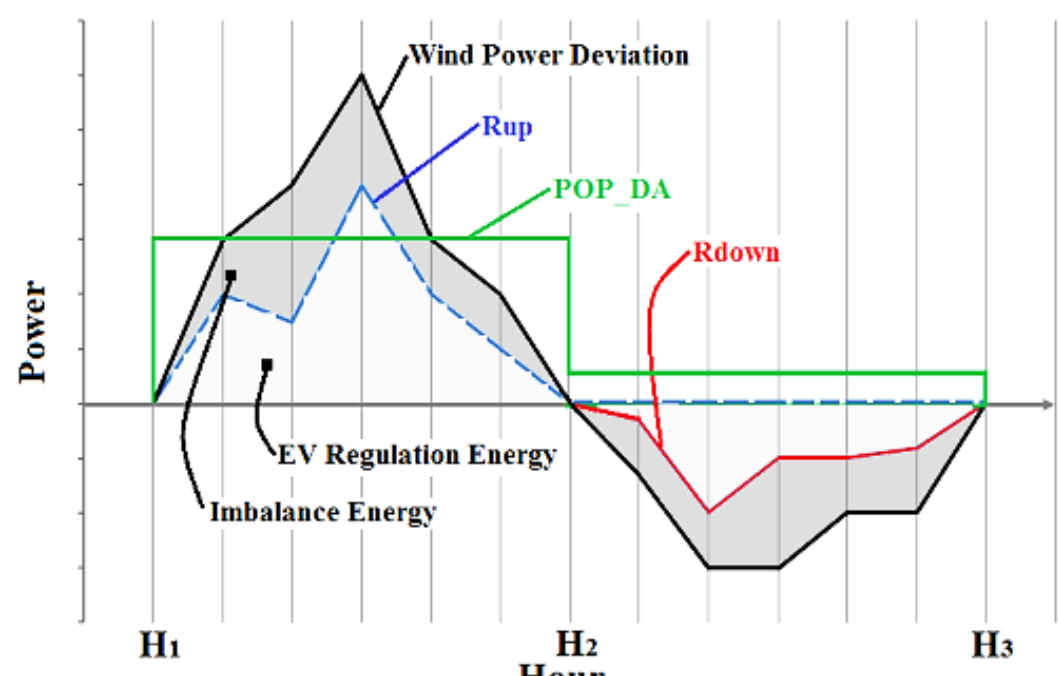

Fig. 3.1 Coordination between EV demand and wind power deviation in energy and regulation market.

The penalties are not imposed for revising the day-ahead power drawn offer quantities [39]. When real-time wind energy exceeds the forecasted day-ahead wind energy, the EV aggregator regulates down with more charging, and vice versa (see Fig. 3.1).

The wind energy deviation between DAM and RTM is compensated by structured regulation, which is provided by the EV aggregator, and unstructured regulation, which is provided by the balancing market as follows:

$$
\Delta P_{s, t, i}^{w}=P_{w, t}^{D A}-P_{w, t, i}^{R T^{s}}=\left(R_{e v, t, i}^{u p^{s}}-R_{e v, t, i}^{d o w n^{s}}\right)+P_{s, t, i}^{i m}
$$

Therefore, the WGenCo can select between regulation and balancing markets based on penalties, energy prices, lack of EVs, and other factors. The WGenCo's payoff in coordination with EV aggregators in this part is: 


$$
\begin{gathered}
\rho_{t}^{D A} P_{w, t}^{D A}-\rho^{P} P_{s, t, i}^{i m}-\rho_{s, t, i}^{R T} \Delta P_{s, t, i}^{w}-\left(\rho_{t}^{R u p} R_{e v, t, i}^{u p^{s}}\right. \\
\left.+\rho_{t}^{R d o w n} R_{e v, t, i}^{d o w n^{s}}\right)
\end{gathered}
$$

Real-time power drawn by the EV aggregator is given by:

$$
P_{s, e v, t, i}^{c h}=P_{O} P_{e v, t}-R_{e v, t, i}^{u p^{s}}+R_{e v, t, i}^{d o w n^{s}}
$$

Hence, the EV aggregator's revenue is obtained by selling ancillary services, as well as selling energy to its clients at a fixed price $\left(\rho^{T}\right)$. In this chapter, the tariff charged to EV clients is assumed to be constant (fixed). The aggregator encourages EV owners to join in by offering an attractive price for charging in comparison with petrol and energy prices.

The EV aggregator's cost is associated with buying energy for EV charging. Hence, the EV aggregator's payoff is represented as:

$$
\begin{array}{r}
-\left(\rho_{t}^{D A}-\rho^{T}\right) P o P_{e v, t}+\left[\rho_{s, t, i}^{R T}-\rho^{T}\right]\left(P o P_{e v, t}-P_{s, e v, t, i}^{c h}\right) \\
+\left(\rho_{t}^{R u p} R_{e v, t, i}^{u p^{s}}+\rho_{t}^{R d o w n} R_{e v, t, i}^{d o w n^{s}}\right)
\end{array}
$$

\subsection{Mathematical Model Formulation}

In this section, the problems of optimal dispatch for three different WGenCo scheduling strategies are formulated and presented. These problems are solved as a two-stage mixed-integer stochastic program [54]. The first-stage variable is decided before stochastic variables with the hourly day-ahead input parameters such as $\rho_{t}^{D A, f}, \rho_{t}^{R u p}$, and $\rho_{t}^{\text {Rdown }}$. The second-stage variable is dependent on 
scenarios with sub-hourly (intra-hourly) RT input parameters such as $\rho_{s, t, i}^{R T, f}, N_{e v, s, t, i}$, and $P_{s, t, i}^{w, f}$.

\subsubsection{Conventional Systems}

The objective function

$$
\begin{aligned}
& \left\langle\operatorname{Max} \sum_{s=1}^{N_{s}} \pi_{s}\left[\sum_{t=1}^{N_{T}} \sum_{w=1}^{N_{w}} \rho_{t}^{D A, f} P_{w, t}^{D A}\right]\right. \\
& -\pi_{s} \cdot\left[\frac{1}{N_{I}} \sum_{i=1}^{N_{I}} \sum_{t=1}^{N_{T}} \sum_{w=1}^{N_{w}} \rho_{s, t, i}^{R T, f} \Delta P_{s, t, i}^{w}\right] \\
& \left.-\pi_{s} \cdot\left[\frac{1}{N_{I}} \sum_{i=1}^{N_{I}} \sum_{t=1}^{N_{T}} \sum_{w=1}^{N_{w}} \rho^{P} \Delta P_{|\Delta| s, t, i}^{w}\right]\right\rangle
\end{aligned}
$$

is to maximize the revenue from selling the day-ahead wind energy minus the cost of energy imbalance [12], [35]. The intra-hour based wind power deviation between real-time and day-ahead schedules is

$$
\Delta P_{s, t, i}^{w}=P_{w, t}^{D A}-P_{w, t, i}^{R T^{s}}=P_{s, t, i}^{i m}
$$

and generation limits are given in

$$
\begin{aligned}
& P_{w, t, i}^{R T^{s}} \leq P_{s, t, i}^{w, f} \\
& P_{w, t}^{D A} \leq P_{t}^{w, f}
\end{aligned}
$$

The following equations are a linear representation of the absolute value of variable $\Delta P_{s, t, i}^{w}$ for the MILP formulation [12]: 


$$
\begin{aligned}
& \Delta P_{|\Delta| s, t, i}^{w}-\Delta P_{s, t, i}^{w} \geq 0 \\
& \Delta P_{|\Delta| s, t, i}^{w}+\Delta P_{s, t, i}^{w} \geq 0 \\
& \Delta P_{|\Delta| s, t, i}^{w}-\Delta P_{s, t, i}^{w} \leq M a_{s, t, i}^{\Delta} \\
& \Delta P_{|\Delta| s, t, i}^{w}+\Delta P_{s, t, i}^{w} \leq M\left[1-a_{s, t, i}^{\Delta}\right]
\end{aligned}
$$

In (3.15), $\mathrm{M}$ is the upper bound of $\left(\Delta P_{|\Delta| s, t, i}^{w}-\Delta P_{s, t, i}^{w}\right)$, and in (3.16), $\mathrm{M}$ is the upper bound of $\left(\Delta P_{|\Delta| s, t, i}^{w}+\Delta P_{s, t, i}^{w}\right)$. These equations are proved in Appendix C.

\subsubsection{WGenCo with the Energy Storage System}

The objective function

$$
\begin{aligned}
& \left\langle\operatorname{Max} \sum_{s=1}^{N_{s}} \pi_{s} \cdot\left[\sum_{t=1}^{N_{T}} \sum_{w=1}^{N_{w}} \rho_{t}^{D A, f} P_{w, t}^{D A}\right]\right. \\
& -\pi_{s} \cdot\left[\frac{1}{N_{I}} \sum_{i=1}^{N_{I}} \sum_{t=1}^{N_{T}} \sum_{w=1}^{N_{w}} \rho_{s, t, i}^{R T, f} \cdot P_{s, t, i}^{i m}\right] \\
& -\pi_{s} \cdot\left[\frac{1}{N_{I}} \sum_{i=1}^{N_{I}} \sum_{t=1}^{N_{T}} \sum_{w=1}^{N_{w}} \rho^{P} P_{|i m|, s, t, i}^{i m}\right] \\
& \left.-\pi_{s} \cdot\left[\frac{1}{N_{I}} \sum_{i=1}^{N_{I}} \sum_{t=1}^{N_{T}} C_{b, s, t, i}^{d c}\right]\right\rangle
\end{aligned}
$$

is to maximize the revenue from selling the day-ahead wind energy minus the cost of energy imbalance and battery charging/discharging costs [39]. The intra-hourbased wind power deviation between real-time and day-ahead schedule is given in 


$$
\Delta P_{s, t, i}^{w}=P_{s, t, i}^{i m}+P_{b, s, t, i}^{d c}-P_{b, s, t, i}^{c h}
$$

The wind power deviation has two terms including imbalance, and battery charging/discharging energy. The following equations are a linear form of the absolute value of variable $P_{s, t, i}^{i m}$ for MILP formulation:

$$
\begin{aligned}
& P_{|i m|, s, t, i}^{i m}-P_{s, t, i}^{i m} \geq 0 \\
& P_{|i m|, s, t, i}^{i m}+P_{s, t, i}^{i m} \geq 0 \\
& P_{|i m|, s, t, i}^{i m}-P_{s, t, i}^{i m} \leq M b_{s, t, i}^{\Delta} \\
& P_{|i m|, s, t, i}^{i m}+P_{s, t, i}^{i m} \leq M\left[1-b_{s, t, k}^{\Delta}\right]
\end{aligned}
$$

where $\mathrm{M}$ is a large positive number [12]. Battery charging/discharging and imbalance power constraints are in

$$
\begin{aligned}
& P_{|i m|, s, t, i}^{i m} \leq \Delta P_{|\Delta| s, t, i}^{w} \\
& 0 \leq P_{b, s, t, i}^{d c} \leq P_{b, \text { Max }}^{d c} I_{b, s, t, i}^{d c} \\
& 0 \leq P_{b, s, t, i}^{c h} \leq P_{b, \text { Max }}^{c h} I_{b, s, t, i}^{c h} \\
& I_{s, b, t, i}^{d c}+I_{s, b, t, i}^{c h} \leq 1
\end{aligned}
$$

Constraints presented in (3.11)-(3.16) are used as well.

The charging/discharging cost depends directly on the depth of discharge (DoD) and the number of cycles to failure of the battery [39]. As the depth of aggregated battery discharge increases, the number of cycles to failure decreases. 
The piecewise linear representation of the concave discharge cost curve of EV batteries in the proposed MIP formulation is shown in

$$
\begin{aligned}
& C_{b, s, t, i}^{d c}=P_{m}^{m i n} \cdot I_{b, s, t, i}^{d c}+\sum_{m=1}^{N_{M}} \varphi_{m} \cdot P_{b, m, s, t, i}^{d c} \\
& \sum_{m=1}^{N_{M}} P_{b, m, s, t, i}^{d c}+P_{m}^{m i n} I_{b, s, t, i}^{d c}=P_{E V, s, t, i}^{d c}
\end{aligned}
$$

\subsubsection{Coordinated EV-Wind Energy Exchange}

The objective function

$$
\begin{aligned}
& \left\langle\operatorname{Max} \sum_{s=1}^{N_{s}} \pi_{s} \cdot\left[\sum_{t=1}^{N_{T}} \sum_{w=1}^{N_{w}} \rho_{t}^{D A, f} P_{w, t}^{D A}\right]\right. \\
& -\pi_{s} \cdot\left[\sum_{t=1}^{N_{T}} \sum_{E V=1}^{N_{E V}}\left(\rho_{t}^{D A, f}-\rho^{T, f}\right) P o P_{e v, t}\right] \\
& +\pi_{s} \cdot\left[\frac{1}{N_{I}} \sum_{t=1}^{N_{T}} \sum_{E V=1}^{N_{E V}} \sum_{i=1}^{N_{I}}\left[\rho_{s, t, i}^{R T, f}-\rho^{T, f}\right]\left(R_{e v, t, i}^{u p^{s}}-R_{e v, t, i}^{d o w n^{s}}\right)\right] \\
& -\pi_{s} \cdot\left[\frac{1}{N_{I}} \sum_{i=1}^{N_{I}} \sum_{t=1}^{N_{T}} \sum_{w=1}^{N_{w}} \rho_{s, t, i}^{R T, f} \cdot \Delta P_{s, t, i}^{w}\right] \\
& \left.-\pi_{s} \cdot\left[\frac{1}{N_{I}} \sum_{i=1}^{N_{I}} \sum_{t=1}^{N_{T}} \sum_{w=1}^{N_{w}} \rho^{P} . P_{|i m|, s, t, i}^{i m}\right]\right\rangle
\end{aligned}
$$

is to maximize the profits of the EV aggregator and the WGenCo according to (3.6) and (3.8). In this strategy, the wind power deviation between DAM and RTM is compensated by regulating the down/up charging power of the EV 
aggregator $\left(R_{e v, t, i}^{\text {down }} n^{s} / R_{e v, s, t, i}^{U P}\right)$ and by the energy imbalance $\left(P_{s, t, i}^{i m}\right)$ provided by the grid, as given by

$$
\Delta P_{s, t, i}^{w}=P_{s, t, i}^{i m}+R_{e v, t, i}^{u p^{s}}-R_{e v, t, i}^{d o w n^{s}}
$$

The energy balance equation for the EV aggregator is given in

$$
E_{e v, t, i}^{s}=E_{e v, t-1, i}^{s}+P_{e v, s, t, i}^{c h} \cdot \Delta t-\left(1-N_{e v, s, t, i}\right) D_{e v, s, t, i}
$$

The EV energy capacity in each intra-hour $\left(E_{e v, t, i}^{s}\right)$ is the EV energy capacity in the prior intra-hour $\left(E_{e v, t, i-1}^{s}\right)$ plus energy charged by drawing power from the grid $\left(P_{e v, s, t, i}^{c h} . \Delta t\right)$ minus energy consumed by EVs while driving. The regulation capacity of the EV aggregator increases when the numbers of charging EVs increase and, vice versa as given in (3.31).

Constraints presented in (3.11)-(3.16) and (3.19)-(3.23) are also used here. Constraints for the EV's POP, capacity to increase the charging rate for regulation down $\left(R_{e v, t, i}^{d o w n}\right)$, capacity to decrease the charging rate for regulation up $\left(R_{e v, t, i}^{u p^{s}}\right)$ are given in

$$
\begin{aligned}
& P_{e} P_{e v, t} \leq P_{e v, c h}^{\max } \cdot N_{e v, s, t, i} \\
& P_{O} P_{e v, t}+R_{e v, t, i}^{d o w n^{s}} \leq P_{e v, c h}^{\max } \cdot N_{e v, s, t, i} \\
& R_{e v, t, i}^{u p^{s}} \leq P_{O} P_{e v, t} \\
& R_{e v, t, i}^{d o w n^{s}} \leq P_{e v, c h}^{\max } \cdot N_{e v, s, t, i} \cdot I_{e v, t, i}^{d o w n^{s}}
\end{aligned}
$$




$$
R_{e v, t, i}^{u p^{s}} \leq P_{e v, c h}^{\max } . N_{E V, s, t, i} I_{e v, t, i}^{u p^{s}}
$$

In

$$
I_{e v, t, i}^{d o w n^{s}}+I_{e v, t, i}^{u p^{s}} \leq 1
$$

the status of regulation down or up is determined. The EV energy constraint is presented in

$$
\begin{aligned}
E_{e v, t}^{\min } \cdot N_{e v, s, t, i} \cdot S O C_{e v}^{\min } \leq E_{E V, s, t, i} & \\
& \leq E_{e v, t}^{\max } \cdot N_{e v, s, t, i} S O C_{e v}^{\max }
\end{aligned}
$$

The constraint

$$
E_{e v, t, i 0}^{s}=E_{e v, t-1, N I}^{s}
$$

imposes limits at the beginning and at the end of each interval of the energy capacity of the EV aggregator. The constraint

$$
E_{e v, T, i}^{s}=E_{e v, t}^{\max } \quad N_{e v, s, t, i} S O C_{e v}^{\max }
$$

specifies the level of SOC to be reached by time $(\mathrm{T})$ for a specified EV client. This constraint is an option for clients to set up the desirable SOC for their EVs at the time of expected commuting (T). For example, the EV client wants to have the battery fully charged (i.e., $100 \%$ SOC) by the departure time (for example, 5:00pm) to go back home. 


\subsection{Case Studies and Numerical Results}

To test the proposed model, a WGenCo with a single wind farm is assumed to participate in a day-ahead energy market. The capacity of the wind farm is 200 MW, which is a relatively small farm compared to the wholesale energy market. The WGenCo is a price-taker; it is not a dominant player in the wholesale energy market. 10,000 scenarios are reduced to just ten using scenario reduction techniques presented in [69]. Fig. 3.2 shows the intra-hourly wind power generation forecasted for these ten scenarios. Fig. 3.3 shows the day-ahead energy price, regulation up/down prices, and intra-hour real time energy price scenarios. Fig. 3.4 shows the intra-hourly EV penetration forecasted for the same ten scenarios. We consider the worst conditions to occur when peak demand and high regulation prices coincide with the lowest penetration of EVs (see Figs. 3.3-3.4. The number of intra-hour intervals is 6 (10 min each).

The maximum EV charging power is assumed to be $7.3 \mathrm{~kW}$, and the energy capacity of each EV is $27.4 \mathrm{kWh}$. Average annual driving distance of an EV is assumed 20,000 $\mathrm{km}$ with an average daily distance of $52.91 \mathrm{~km}$. The required energy for an EV is $9 \mathrm{kWh} /$ day with an average of $5.87 \mathrm{~km} / \mathrm{kWh}$ [35], [39], [43]. In this chapter, the fixed charging tariff is assumed to be $\$ 0.01 / \mathrm{kWh}[12]$. We assume that the required energy for driving in one direction is the same as that of returning to the starting point. For the EV aggregator, we consider two EV penetration scenarios 1,000 and 10,000 EVs. The cycle efficiency is $83.6 \%$ for a charging/discharging efficiency of $95 \%$ [35, 65]. The EV fleet has its own commute time based on the region, city, traffic patterns, etc. The number of EV fleets is assumed to be one with commute intervals between 7 A.M and 9 A.M, and between 5 P.M. and 8 P.M. However, the equations provided in the chapter are 
general and can be used for any number of EV fleets. In this chapter, 100\% SOC is considered for departure times to represent the worst case scenario.

The capacity of a battery bank of the ESS is assumed to be similar to the capacity of 10,000 EVs. The current price of a complete battery pack is $\$ 600 / \mathrm{kWh}$.

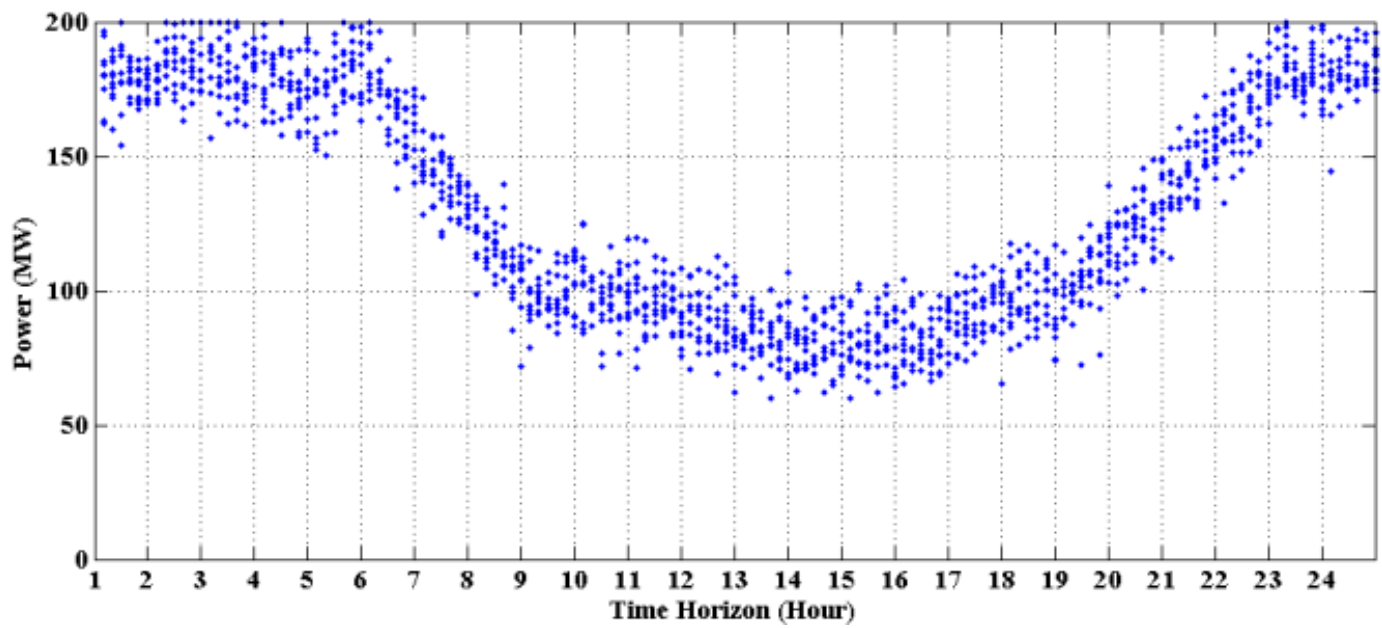

Fig. 3.2 The intra-hourly wind power generation forecasted for ten scenarios.

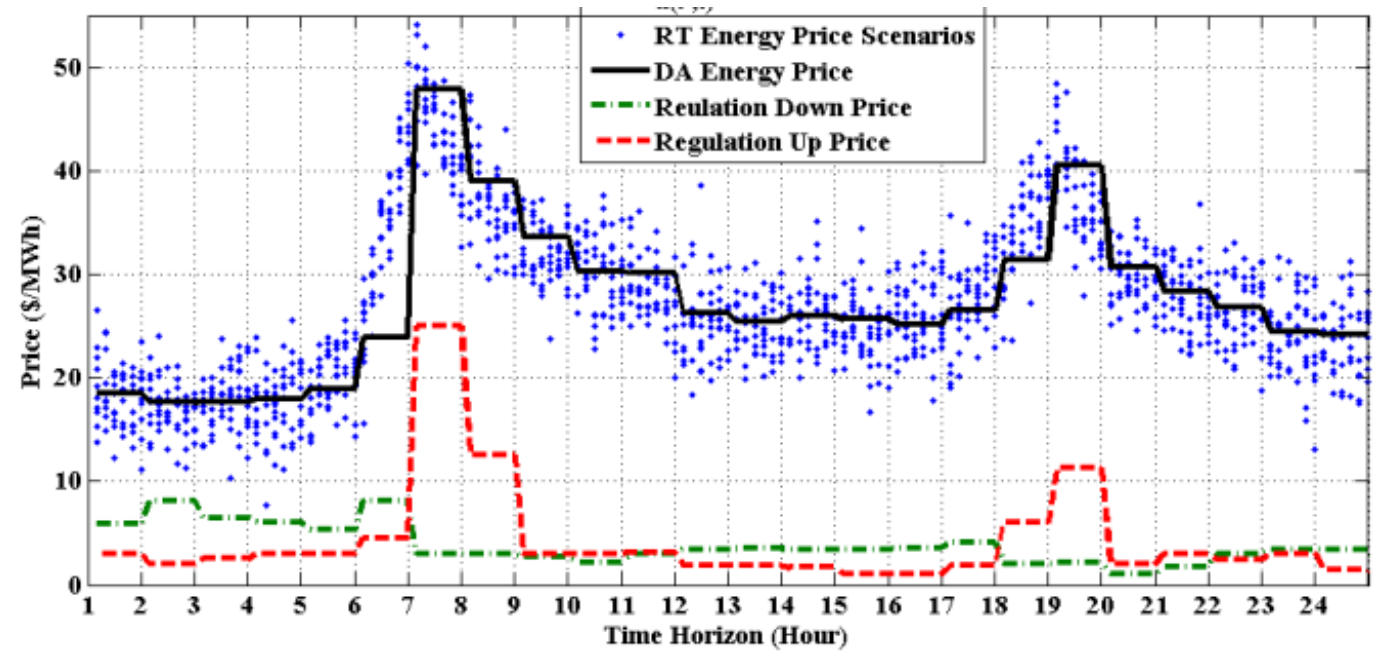

Fig. 3.3 The day-ahead energy price, regulation up/down prices, and intra-hour real time energy price scenarios. 


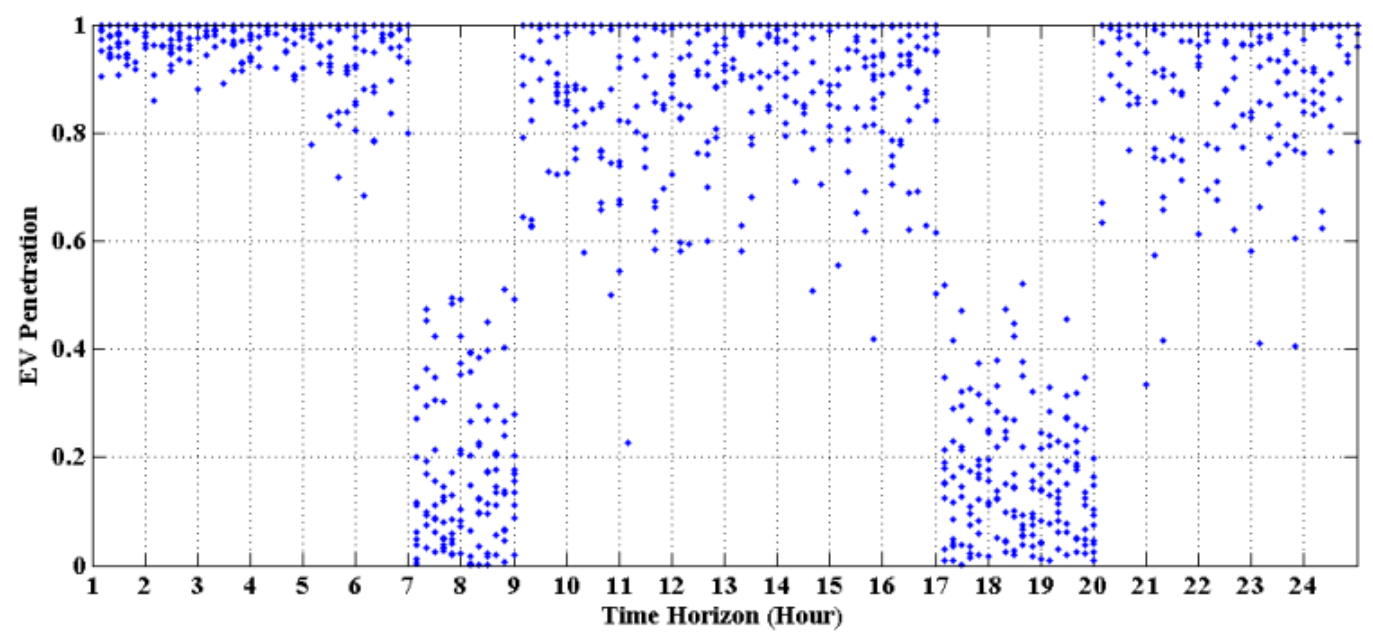

Fig. 3.4 The intra-hourly EV penetration forecasted for ten scenarios.

Four cases are considered for the investigation of two important issues: the payoff, and generation and demand dispatch. The four cases are defined as follows:

- Case A-Conventional systems: The WGenCo without ESS participates in the energy market.

- Case B-ESS-wind: The WGenCo with ESS participates in the energy market.

- Case C-1K-EV-Wind: The WGenCo in coordination with 1,000 EVs participates in the energy and regulation markets.

- Case D-10K-EV-Wind: The WGenCo in coordination with 10,000 EVs participates in the energy and regulation markets.

\subsubsection{Payoff Analysis}

Table 3.1 shows the total WGenCo's payoffs for all cases when the penalty price is $\$ 30 /$ MWh. The total payoff for Case $\mathrm{D}$ is $\$ 79,888.98$, while the expected payoffs in cases $\mathrm{A}$ and $\mathrm{B}$ are $\$ 77,023.94$ and $\$ 77,064.63$, respectively. The 
difference between the two payoffs in case D and case $\mathrm{A}$ is $\$ 2,869.04(3.72 \%)$, while the difference between the two payoffs in Case B and Case A is just $\$ 40.69$ $(0.05 \%)$. It is clear that using the battery storage at a penalty price of $\$ 30 / \mathrm{MWh}$ is not affordable. The EV penetration impact on the payoffs is obvious when comparing cases C and D. Table 3.1 shows that the payoff in Case D exceeds that of Case C by $1.86 \%$.

Table 3.1

WGenCo's Payoffs In Different Cases at the $\$ 30$ Penalty Price

\begin{tabular}{ccccc}
\hline Cases & Case A & Case B & Case C & Case D \\
\hline DA Energy Sale Revenue $(\$)$ & 81132.56 & 81177.86 & 83395.12 & 99763.90 \\
\hline DA Revenue Adjustment $(\$)$ & -875.06 & -702.45 & -2307.46 & -17520 \\
\hline Imbalance Charge $(\$)$ & -3233.56 & -3071.77 & -2491.57 & -589.20 \\
\hline Regulation Cost $(\$)$ & - & - & -195.60 & -1620.61 \\
\hline Discharging Cost $(\$)$ & - & -339.01 & - & - \\
\hline Payoff $(\$)$ & 77023.94 & 77064.63 & 78400.48 & 79888.98 \\
\hline
\end{tabular}

Tables 3.2-3.5 and Figs. 3.5-3.7 show the impact of the penalty price on the WGenCo's payoffs for different cases. It is clear that with the increasing penalty price, the day-ahead energy sale revenue and the total payoff decrease with more conservative day-ahead generation offers. However, with the EV-wind coordination, the total payoff with respect to the penalty price remains almost constant (see Fig. 3.5). For example, while penalty price changes from $\$ 10 / \mathrm{MWh}$ to $\$ 150 / \mathrm{MWh}$, the total payoff in cases D, C, B, and A decreases by $2.21 \%$, $7.42 \%, 8.02 \%$ and $10.01 \%$, respectively. It is clear that the total payoff under variable penalty price is more sustainable (stable) with the 10K-EV-Wind coordination (see Fig. 3.5). 
Comparing results presented in Tables 3.2-3.4 shows that the difference between the two payoffs in Case D and Case $\mathrm{A}$ at the penalty price of $\$ 10 / \mathrm{MWh}$ is $\$ 384(0.4 \%)$, while this difference is $\$ 6683(8.4 \%)$ at the penalty price of $\$ 150 /$ MWh. Therefore, the effectiveness of coordinating EVs with wind generation becomes more apparent for penalty prices greater than $\$ 20 /$ MWh (see Fig. 3.5).

Comparing Case B with cases $\mathrm{C}$ and A shows that the total payoff in Case B is greater than in Case A for penalty prices greater than $\$ 40 / \mathrm{MWh}$. The total payoff in Case B is lower than in Case C although they get closer with higher penalty prices (see Fig. 3.5), since the battery discharging cost is more affordable under higher penalty prices.

Comparing cases $\mathrm{D}$ and $\mathrm{C}$ shows that the appropriate capacity of the EV aggregator is an important factor in coordinating the EVs and WGenCos. Benefits of the use of EV aggregators of sufficient capacity include higher total payoffs, lower imbalance charges and less conservative day-ahead generation offers. However, a smaller number of EVs offer better results in comparison with cases A and B.

Fig. 3.6 shows imbalance charges provided by the balancing market versus penalty prices. It is clear that the imbalance charge in Case D is less than in the other cases. Fig. 3.7 demonstrates that an increase in penalty price decreases the EV regulation cost for the WGenCO (or the EV regulation profitability for the EV aggregator) when wind deviations decrease, thus the EV regulation contribution is lower. The battery charging/discharging cost for Case B would increase with the increase in the penalty price, because imbalance charges imposed by the balancing market are more expensive than the battery charging/discharging cost under higher penalty prices; thus the ESS contribution is higher. 
Table 3.2

Impact of the Penalty Price on WGenCo's Payoffs In Case A

\begin{tabular}{ccccc}
\hline Penalty Price (\$/MWh) & 10 & 50 & 100 & 150 \\
\hline \hline DA Energy Sale Revenue $(\$)$ & 78966.02 & 79304.49 & 76935.06 & 75953.17 \\
\hline DA Revenue Adjustment $(\$)$ & 4853.88 & -1375.28 & -720.16 & -545.94 \\
\hline Imbalance Charge (\$) & -3136.93 & -2332.88 & -2411.29 & -2745.66 \\
\hline Payoff $(\$)$ & 80682.97 & 75596.32 & 73803.61 & 72661.56 \\
\hline
\end{tabular}

Table 3.3

Impact of the Penalty Price on WGenCo's Payoffs In Case B

\begin{tabular}{ccccc}
\hline Penalty Price $(\$ /$ MWh $)$ & 10 & 50 & 100 & 150 \\
\hline \hline DA Energy Sale Revenue $(\$)$ & 78721.16 & 79605.04 & 79097.86 & 77505.53 \\
\hline DA Revenue Adjustment $(\$)$ & 4046.73 & -1018.64 & -272.77 & -149.45 \\
\hline Imbalance Charge (\$) & -2330.48 & -1763.76 & -939.59 & -920.54 \\
\hline Discharging Cost $(\$)$ & -1.49 & -987.91 & -3272.19 & -2254.82 \\
\hline Payoff $(\$)$ & 80435.92 & 75834.72 & 75907.64 & 74329.83 \\
\hline
\end{tabular}

Table 3.4

Impact of the Penalty Price on WGenCo's Payoffs In Case D

\begin{tabular}{ccccc}
\hline Penalty Price $(\$ / \mathrm{MWh})$ & 10 & 50 & 100 & 150 \\
\hline \hline DA Energy Sale Revenue $(\$)$ & 103290.28 & 98285.83 & 96980.28 & 96154.44 \\
\hline DA Revenue Adjustment $(\$)$ & -21730 & -16370 & -15380 & -14760 \\
\hline Imbalance Charge $(\$)$ & -492.93 & -492.99 & -486.48 & -560.03 \\
\hline Regulation Cost $(\$)$ & -2339.87 & -1650.21 & -1543.62 & -1489.86 \\
\hline Payoff $(\$)$ & 81067.35 & 79775.13 & 79568.18 & 79344.94 \\
\hline
\end{tabular}




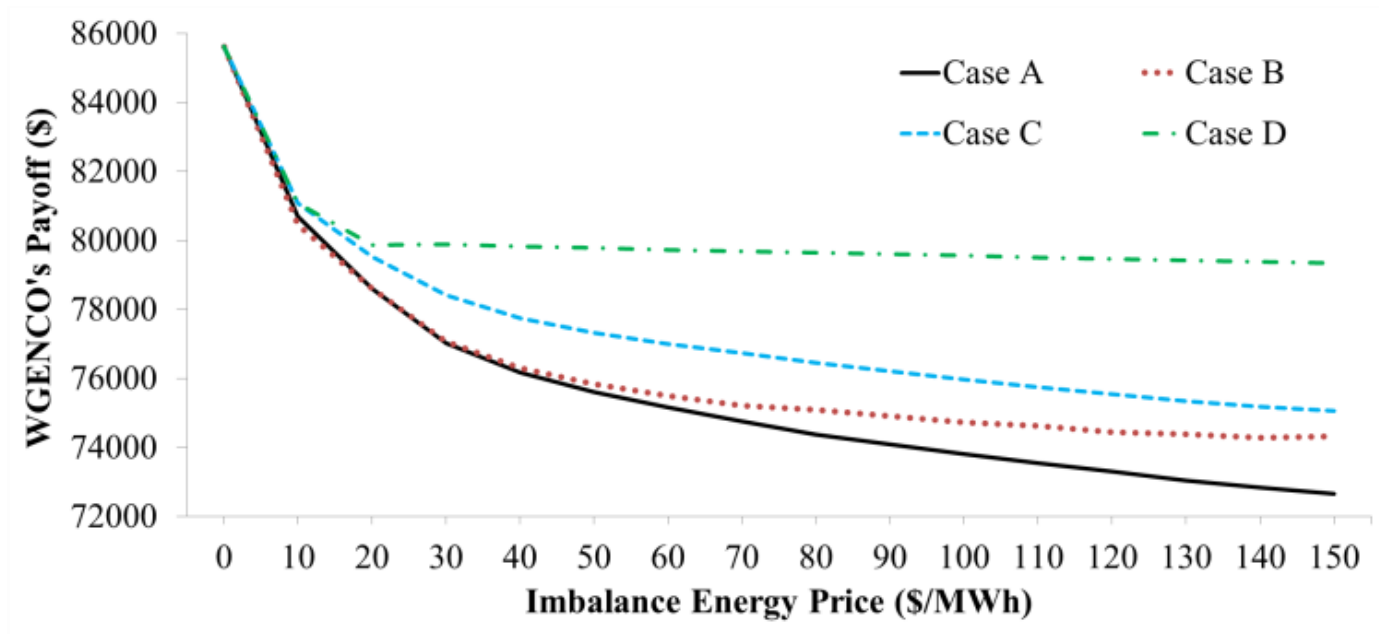

Fig. 3.5 The WGenCo's payoff versus penalty prices.

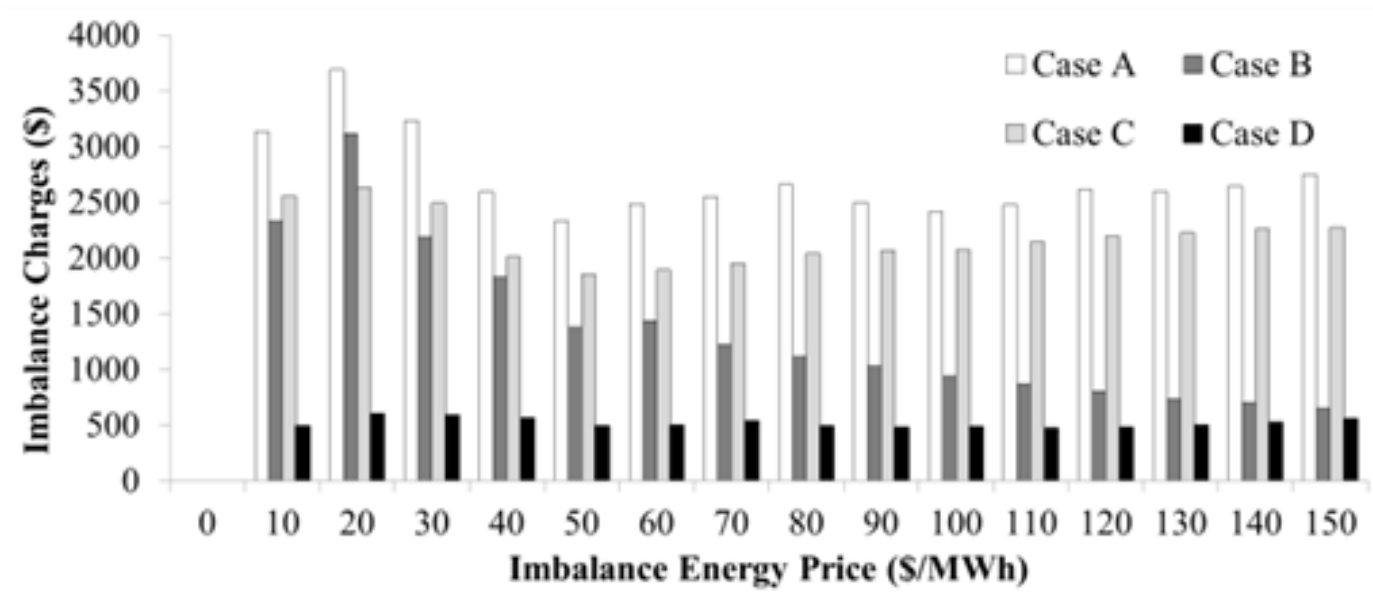

Fig. 3.6 Imbalance charges provided by balancing market versus penalty prices

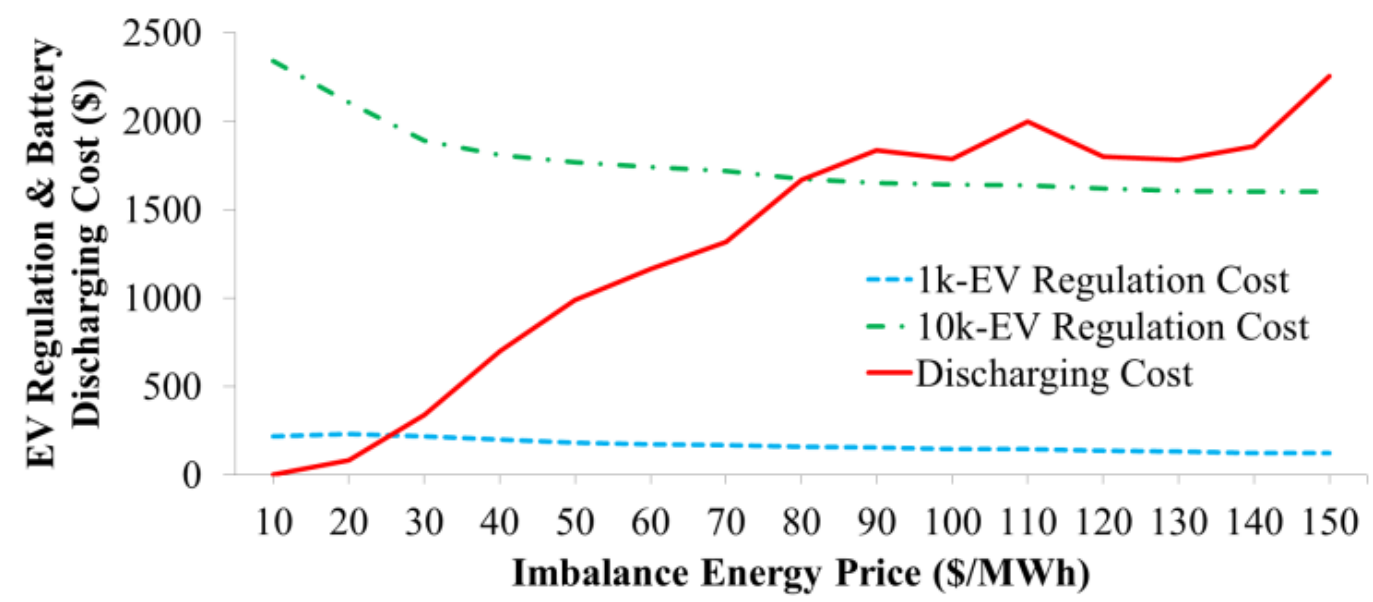

Fig. 3.7 EV regulation and battery discharging cost versus penalty prices. 


\subsubsection{Demand and Generation Dispatch analysis}

Wind power generation and EV load demand dispatch in Case D at penalty prices of $\$ 10 / \mathrm{MWh}$ and $\$ 150 / \mathrm{MWh}$ are shown in Figs. 3.8-3.9, respectively. These figures show day-ahead wind power $\left(\mathrm{Pw}_{\mathrm{w}} \mathrm{DA}\right)$, real-time wind power $\left(\mathrm{Pw}_{\mathrm{w}}\right.$ RT), wind power deviation $(\Delta P w)$, day-ahead EV charging schedule (POP-DA), EV regulation up/down, and energy imbalance provided by the balancing market $\left(P^{i m}\right)$. Figs. 3.10 and 3.11 show wind power generation and battery bank dispatch in Case B at penalty prices of $\$ 10 \mathrm{MWh}$ and $\$ 150 / \mathrm{MWh}$, respectively.

It can be seen that the Pw-RT schedules in Figs. 3.8, 3.9, and 3.10 look very similar, but the schedule in Fig. 3.11 differs noticeably. This demonstrates that the penalty price increase has a greater impact in Case B than in Case D. For instance, in Table 3.5, the total Pw-RT per day in cases B and D are 2,977 MWh, and 3,111 MWh, respectively, at the penalty price of $\$ 150 / \mathrm{MWh}$. However, the total $\mathrm{Pw}-\mathrm{RT}$ per day in cases $\mathrm{B}$ and $\mathrm{D}$ are the same for the $\$ 10 / \mathrm{MWh}$ penalty price. This demonstrates that the effectiveness of the coordinated EV-wind energy exchange becomes more apparent when penalty prices are higher. From Table 3.5, it can also be observed that the total $\mathrm{Pw}-\mathrm{RT}$ in Case $\mathrm{D}$ remains almost unchanged irrespective of the penalty price.

If we now compare the total $\Delta P w$ per day for all cases under the $\$ 10 / \mathrm{MWh}$ penalty price, we find that this parameter is much higher in Case D (this can be attributed to the less conservative day-ahead generation offers). We can also find that the total $P^{i m}$ per day under any penalty price is smaller in Case D than in all other cases (this fact is particularly apparent under the $\$ 10 / \mathrm{MWh}$ penalty price). 
Results presented in Figs. 3.8, 3.9 and Table 3.5 also demonstrate that the increase in the penalty price decreases the total up/down EV regulation contributions - the total up/down EV regulation at $\$ 10 / \mathrm{MWh}$ and $\$ 150 / \mathrm{MWh}$ penalty prices are 761.9 MWh and 584.1 MWh, respectively.

Results presented in Figs. 3.10, 3.11 and Table 3.5 show that the increase in penalty price leads to higher battery charging/discharging costs (Case B). The total charging/discharging costs at penalty prices of $\$ 10 / \mathrm{MWh}$ and $\$ 150 / \mathrm{MWh}$ are 4.7 MWh and 69.8 MWh, respectively. Battery discharge power increases under the higher penalty prices, because the battery charging/discharging cost is more affordable than imbalance charges imposed by the balancing market under higher penalty prices.

Table 3.5

Total Real-Time Wind Power Generation, Wind Power Deviation and Energy Imbalance per Day

\begin{tabular}{|c|c|c|c|c|c|}
\hline Total (MWh) & Penalty Price & Case A & Case B & Case $\mathrm{C}$ & Case D \\
\hline Pw-RT & \multirow{3}{*}{$10(\$ / M W h)$} & 3138 & 3138 & 3138 & 3137 \\
\hline$\Delta P w$ & & 313.69 & 216.56 & 325.12 & 811.24 \\
\hline$P^{i m}$ & & 313.69 & 211.86 & 255.42 & 49.29 \\
\hline Pw-RT & \multirow{3}{*}{$100(\$ / \mathrm{MWh})$} & 2896 & 2990 & 2976 & 3116 \\
\hline$\Delta P w$ & & 24.11 & 79.16 & 60.58 & 609.2 \\
\hline$P^{i m}$ & & 24.11 & 9.39 & 20.74 & 4.86 \\
\hline $\mathrm{Pw}_{\mathrm{w}} \mathrm{RT}$ & \multirow{3}{*}{$150(\$ / \mathrm{MWh})$} & 2867 & 2977 & 2949 & 3111 \\
\hline$\Delta P w$ & & 18.30 & 73.59 & 46.1 & 588.8 \\
\hline$P^{i m}$ & & 18.30 & 4.317 & 15.167 & 3.733 \\
\hline
\end{tabular}




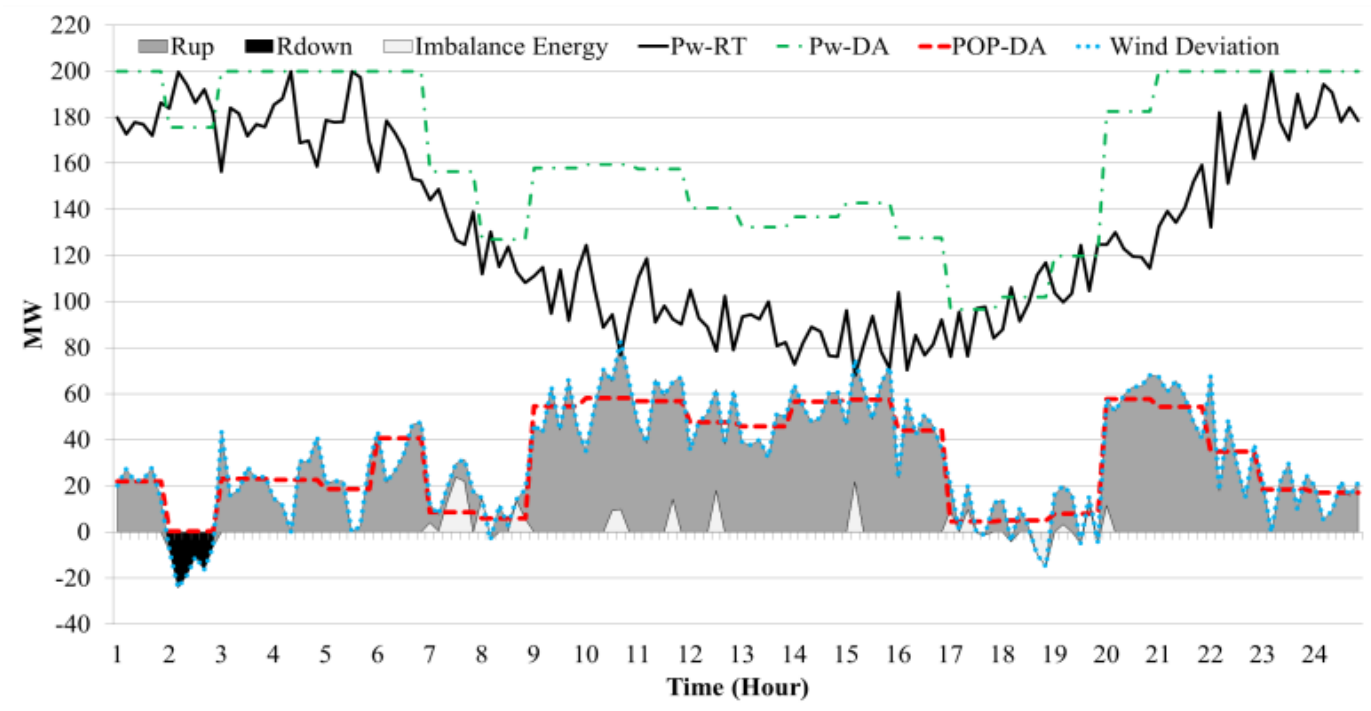

Fig. 3.8 Wind power generation and EV demand schedule in Case D under the $\$ 10 / \mathrm{MWh}$ penalty price.

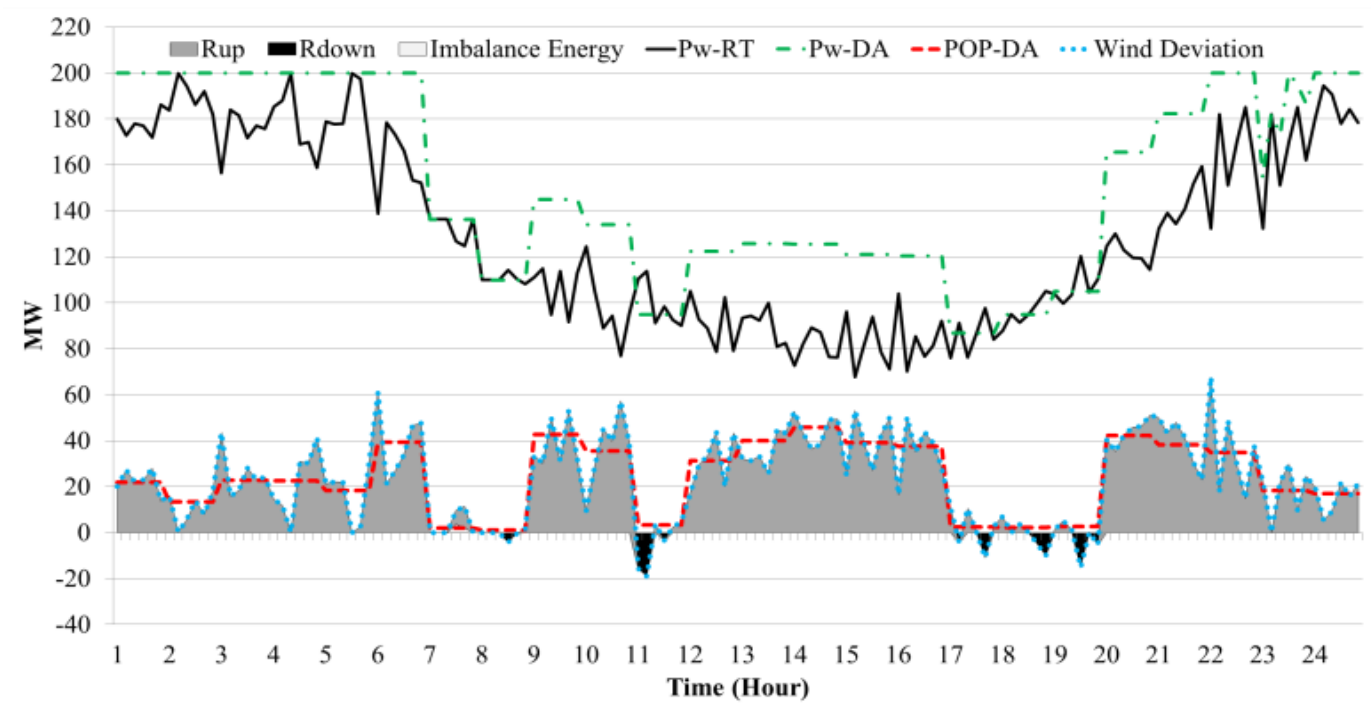

Fig. 3.9 Wind power generation and EV demand schedule in Case D under the $\$ 150 /$ MWh penalty price. 


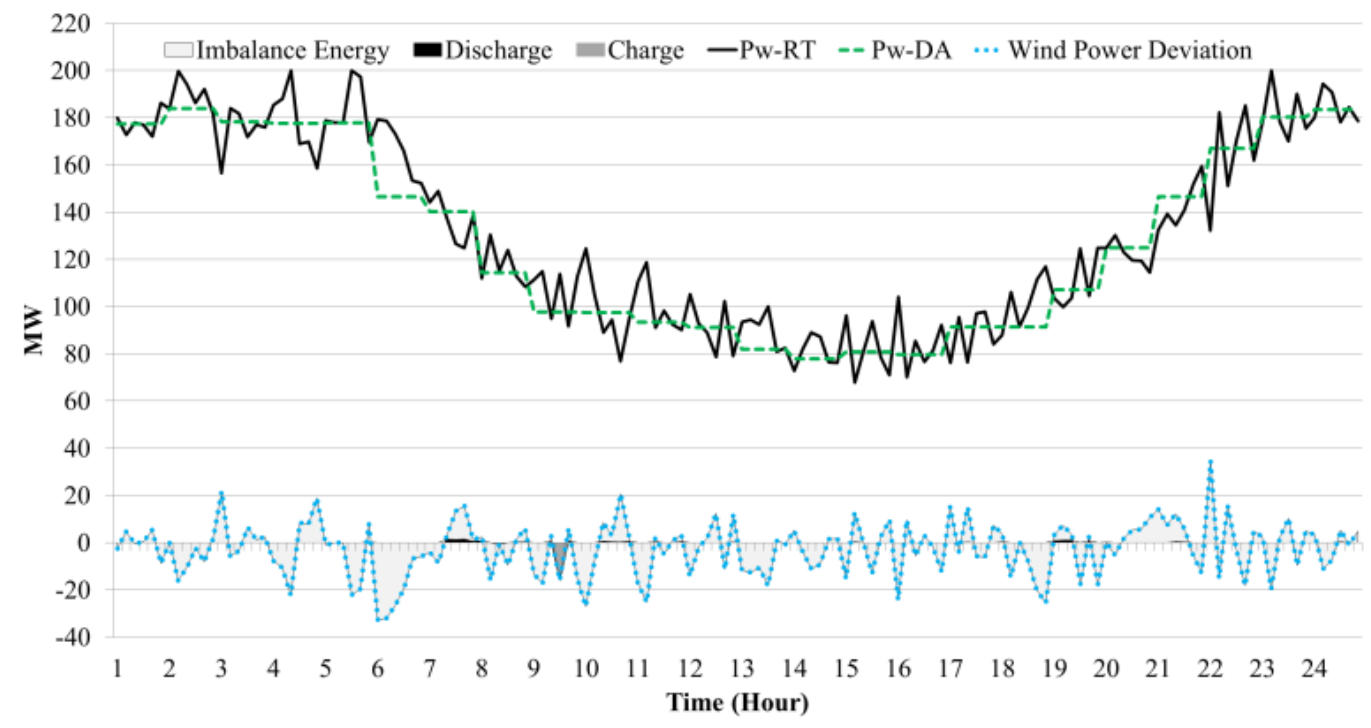

Fig. 3.10 Wind power deviation and battery energy storage profile in case B at 10 $\$ / \mathrm{MWh}$ penalty price

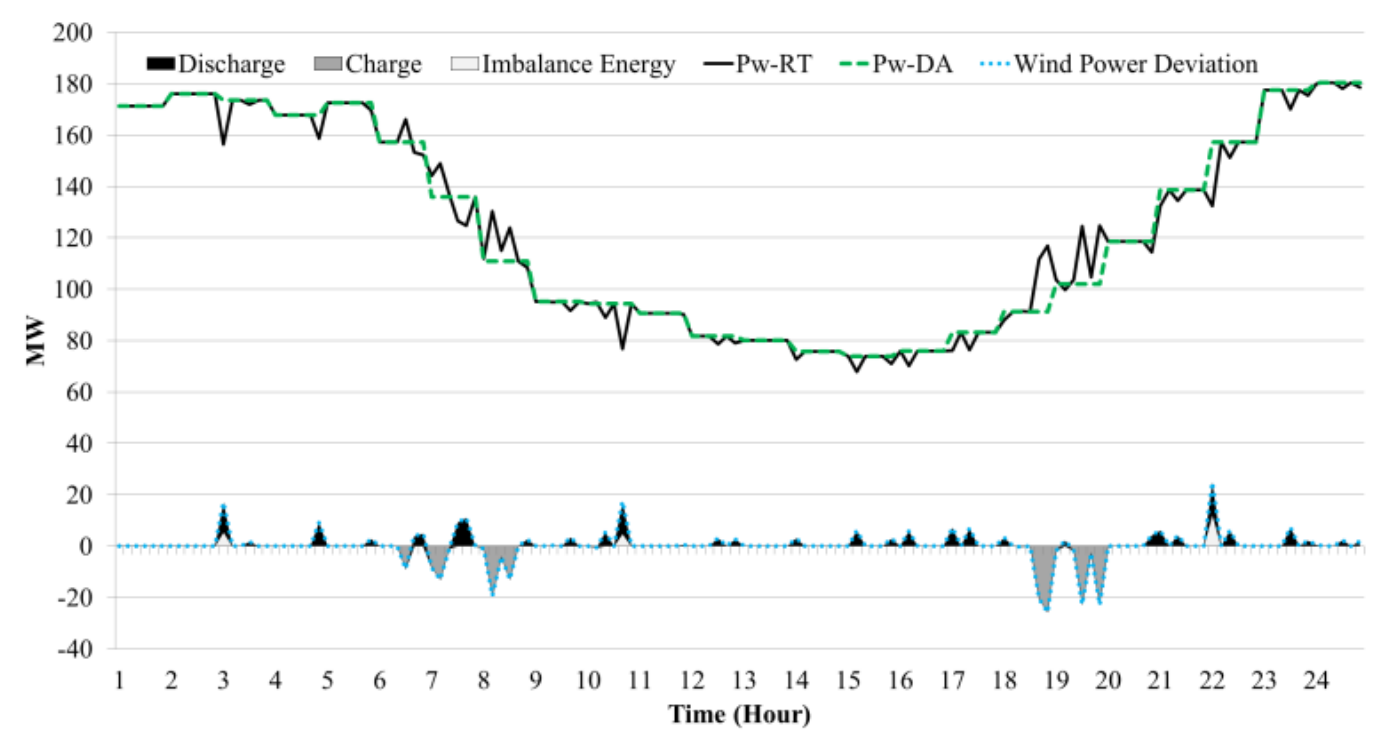

Fig. 3.11 Wind power generation schedule and battery energy storage profile in case B at $150 \$ /$ MWh penalty price 


\subsection{Summary}

Effective coordination between a WGenCo participating in the short-term electricity market and an EV aggregator participating in the energy and ancillary service markets increases the WGenCo's competitiveness and mitigates wind and EV energy imbalance threats. This chapter has introduced a stochastic optimal scheduling strategy. The strategy has been demonstrated on conventional systems (WGenCo without storage), WGenCo with ESS, and a power system with a coordinated EV-Wind energy exchange. The proposed strategy has been developed using model based-optimal decision making. It offers flexibility in selecting between the balancing, regulation services, and/or ESS for a WGenCo to compensate for wind power deviations. Comparisons of the coordinated EV-Wind energy exchange with the other two cases reveal that

- the coordinated EV-wind energy exchange ensures that the WGenCo payoff remains constant under changing penalty prices;

- the effectiveness of the coordinated EV-wind energy exchange becomes more apparent under higher penalty prices;

- $\quad$ benefits offered by EV aggregators of sufficient capacity include higher total payoffs, lower imbalance charges and less conservative day-ahead generation offers. However, a smaller number of EVs offer better results in comparison with the other two cases;

- $\quad$ the total energy imbalance adjusted by the balancing market decreases extremely under the coordinated EV-wind energy exchange. 



\section{Chapter 4}

\section{Pool Strategy of a Single Firm in}

\section{Coordination with EV Load Aggregators}

\subsection{Introduction}

It is vital for a firm (a producer including CGenCos and WGenCos) and the market operator to review equilibrium analysis to investigate new players influenced in the future smart grid $[13,56]$. The WGenCos participate in electricity markets despite their uncertainty to maximize the expected payoff, similar to the other market producers with consideration of WGenCo as pricemaker market player [11]. Also, some new market players (e.g. EV aggregators) have to compete with other market players while motivating the consumers to take part in the market [11-13].

However, the highest benefits for EV load aggregators are expected through participation in ancillary services [39]. The EV load aggregator meets to challenge 
of considering both minimum tariff to motivate EV owners to take part in the market and high probability to be competitive on its own. Therefore, the determination of an optimal charging tariff is necessary to maximize the aggregator's profit and keep the current EV owners and attract new customers. The EV charging tariff can affect prices and the market outcomes.

This chapter investigates EV aggregator as price maker which is in the generation portfolio of a strategic producer including WGenCo and CGenCos. The main contributions of the chapter are:

- The development of an optimal bidding/offering strategy for the EV load aggregator providing the energy and ancillary services in coordination with a strategic producer in a pool-based electricity market with endogenous formation of DA and RT prices, and EV aggregator tariff.

- Optimization of EV charging profile with consideration of both endogenous fixed-rate and Time of Use (ToU) tariffs.

- The proposed formulation of a stochastic intra-hour bilevel optimization problem given by an MPEC including an upper-level problem and three lower-level problems for the sake of a) the strategic firm's profit maximization, and b) DA and RT social welfare, and EV owner's battery energy maximization, respectively.

- The analysis of the impact of EV numbers, both fixed-rate and ToU tariff on the price and market outcome.

- Consideration of the uncertainties related to wind speed, and EV owners' behavior based on driving patterns.

The rest of this chapter is organized as follows. Section 4.2 discusses the market framework. Section 4.3 provides a mathematical model formulation. Test of the 
proposed market model through case studies is described in Section 4.4. Finally, Section 4.5 concludes, summarizing the chapter.

\subsection{Proposed Market Framework}

Two levels of market framework are introduced in this thesis which are wholesalelevel and EV-level shown in Fig. 4.1. At the wholesale-level, a strategic firm including CGenCos, WGenCos, and EV aggregators submits supplyoffers/demand-bids to the MO to participate in the day-ahead and real-time market directly. The MO runs the day-ahead market clearing process to determine day-ahead price, and power production schedules of CGenCos, WGenCos, LSEs and EVs. Also, the real-time market is cleared for each scenario based on achieved day-ahead data to determine real-time prices, regulation capacities, and wind power and load curtailments.

At the EV-level, EV owners connect to the EV aggregator in order to take part in the market indirectly. The EV aggregator determines maximum EV energy capacity and optimal charging tariff based on achieved day-ahead and real-time data.

The model assumptions are as follows:

1. The EV aggregator participates as dispatchable loads in the market by submitting strategic bid and offer prices to the day-ahead and the realtime markets, respectively at the wholesale-level. At the EV-level, EV owners connect to the EV aggregator to participate in the market for increasing the negotiation power of EV customers. Therefore, each EV fleet or owner is assigned to an EV aggregator through a contract [11]. The EV charging energy, charging tariff, and up and down regulation directly affect the strategic producer's expected profit. The amount of 
regulation contracted is the total amount by which power can deviate from a baseline level (or POP). It is assumed that the EV aggregator can deviate from the day-ahead power-drawn (or POP) to balance energy by reducing or increasing their charging rate with consideration of $\mathrm{EV}$ aggregator energy constraints [6].

2. WGenCo submits strategic offer prices to the day-ahead and the real-time markets. Moreover, the wind power production excess/shortage, and curtailment power influence the strategic producer's expected profit [13].

3. CGenCo participates as dispatchable units in the market by submitting strategic offer prices to the day-ahead and the real-time markets, respectively. The energy production cost functions are assumed to be linear [99].

4. The LSEs submit bid prices for energy and curtailment (to be elastic) to the day-ahead and real market but not strategically [13, 65,93].

5. A transmission network is neglected for simplicity.

6. The price scheme used is the same as the scheme presented in [99]. Each generating unit is paid for its scheduled power production and EV loads are charged for their power consumption in the day-ahead market at the price $\rho_{t}^{D A}$. Additionally, each generating unit and $\mathrm{EV}$ aggregator is paid/charged for its regulation up/down at the prices $\rho_{t, i}^{R T, s}$ as repurchased prices [99]. 


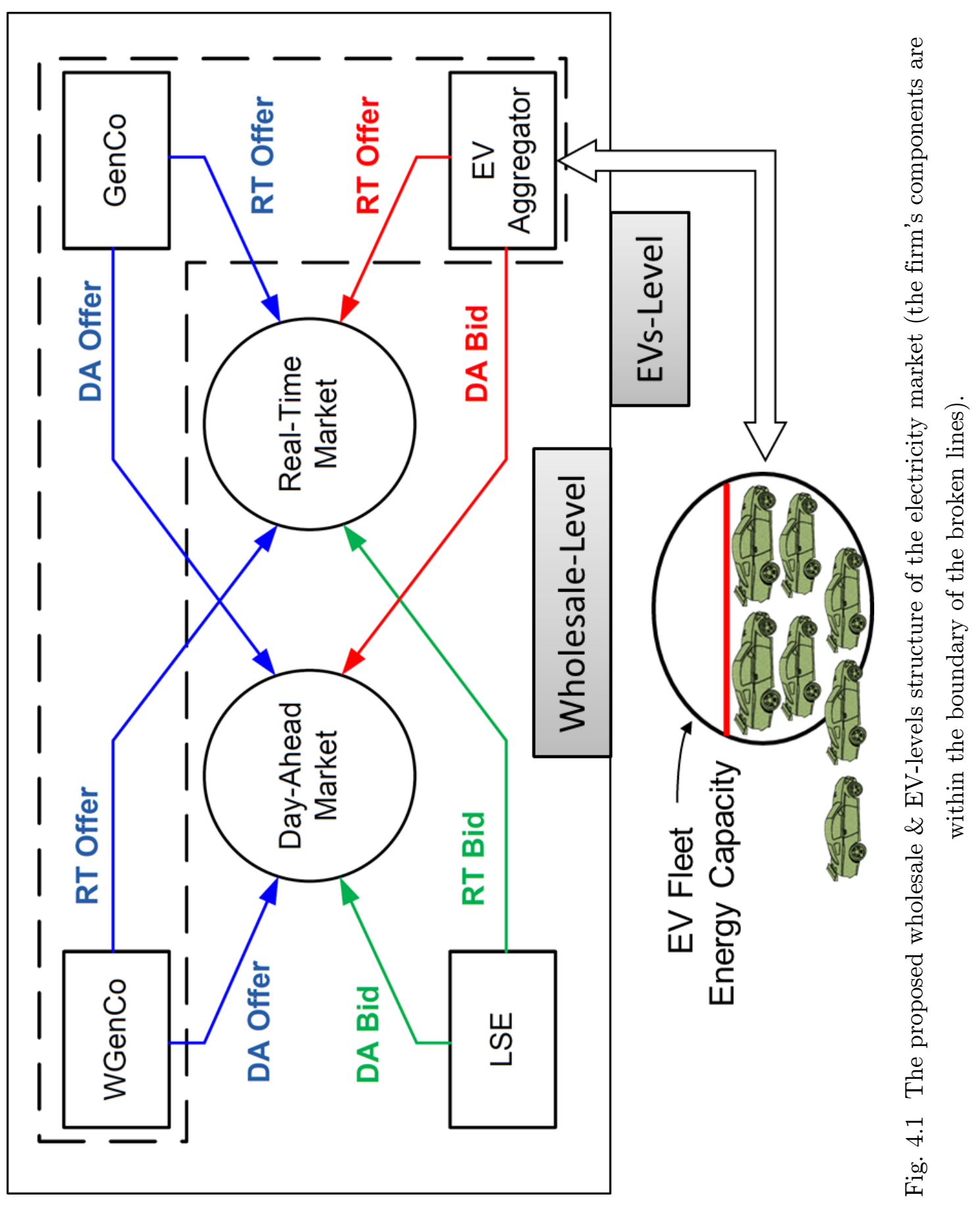




\subsection{Mathematical Model Formulation}

A bilevel (hierarchical) model is used in this chapter to model the behaviour of a strategic firm.

\subsubsection{Bilevel Model}

A stochastic bilevel model is taken into account where an upper-level problem corresponds to a strategic firm's profit maximization, while the lower-level problems correspond to markets clearing for maximization of social welfare as shown in Fig. 4.2. The lower-level problems consist of the day-ahead market, realtime market, and EV energy market clearing which aim to maximize the social welfare and are subject to the power balance, and power limits.

The day-ahead lower level determines the day-ahead price and the power production/consumption quantities which directly affect the firm's expected profit in the upper-level problem, and EV energy and real-time market clearing in the lower-level problems. The real-time lower level presents the clearing of the realtime market based on EV numbers and wind power production scenarios which directly affect the firm's expected profit in the upper-level problem, and EV energy market in the lower-level problems. The EV energy lower level determines optimal charging tariff $\left(\rho^{T}\right)$ based on achieved day-ahead and real-time data which directly affect the firm's expected profit in the upper-level problem. The strategic offering and decisions made by a strategic firm in the upper-level problem influence the market clearing outcomes in the lower-level problems.

The mathematical formulation of electricity markets is developed based on offering/bidding strategies given in $[13,93]$. 


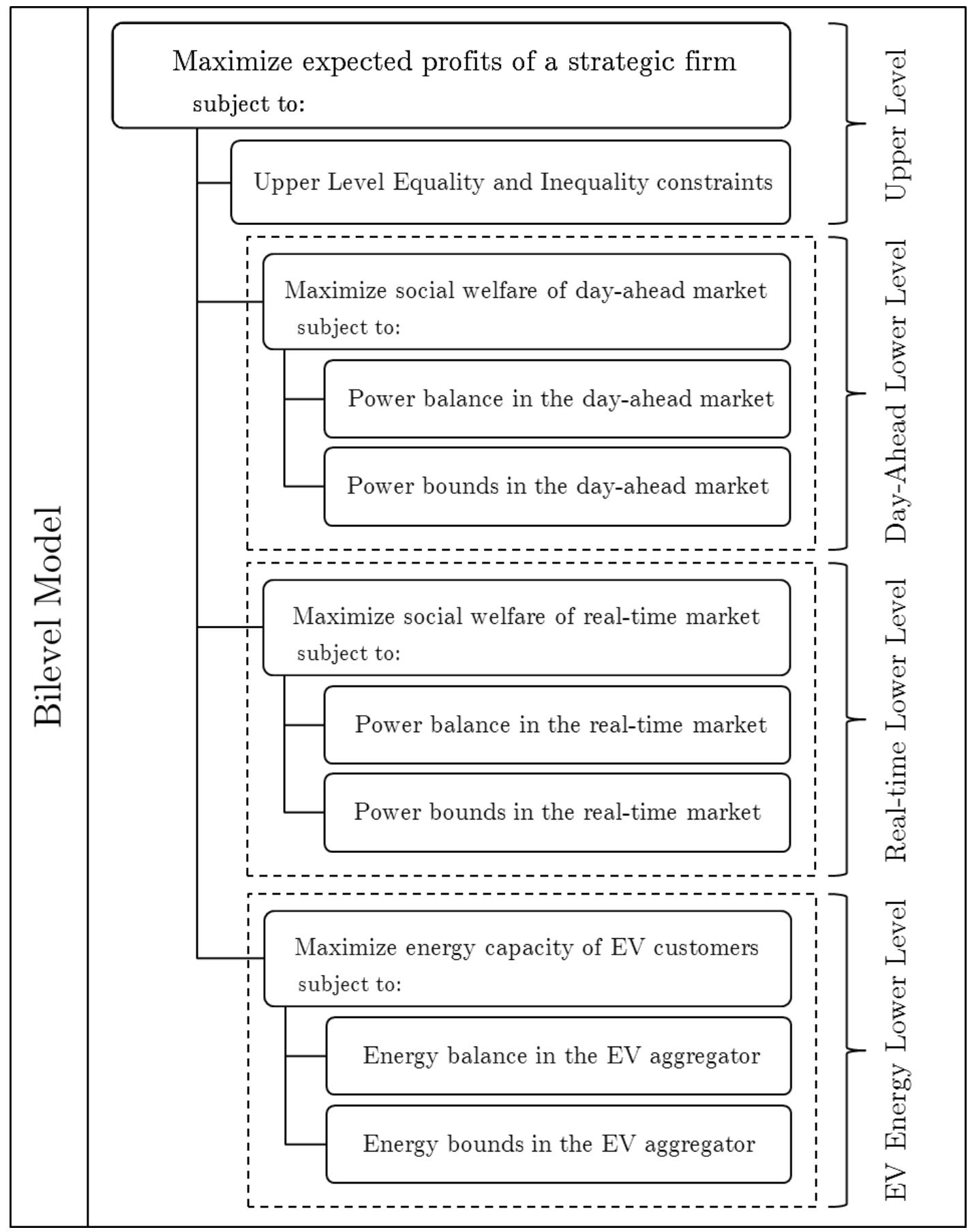

Fig. 4.2 Bilevel structure of the proposed wholesale \& EV-levels structure of the electricity market. 


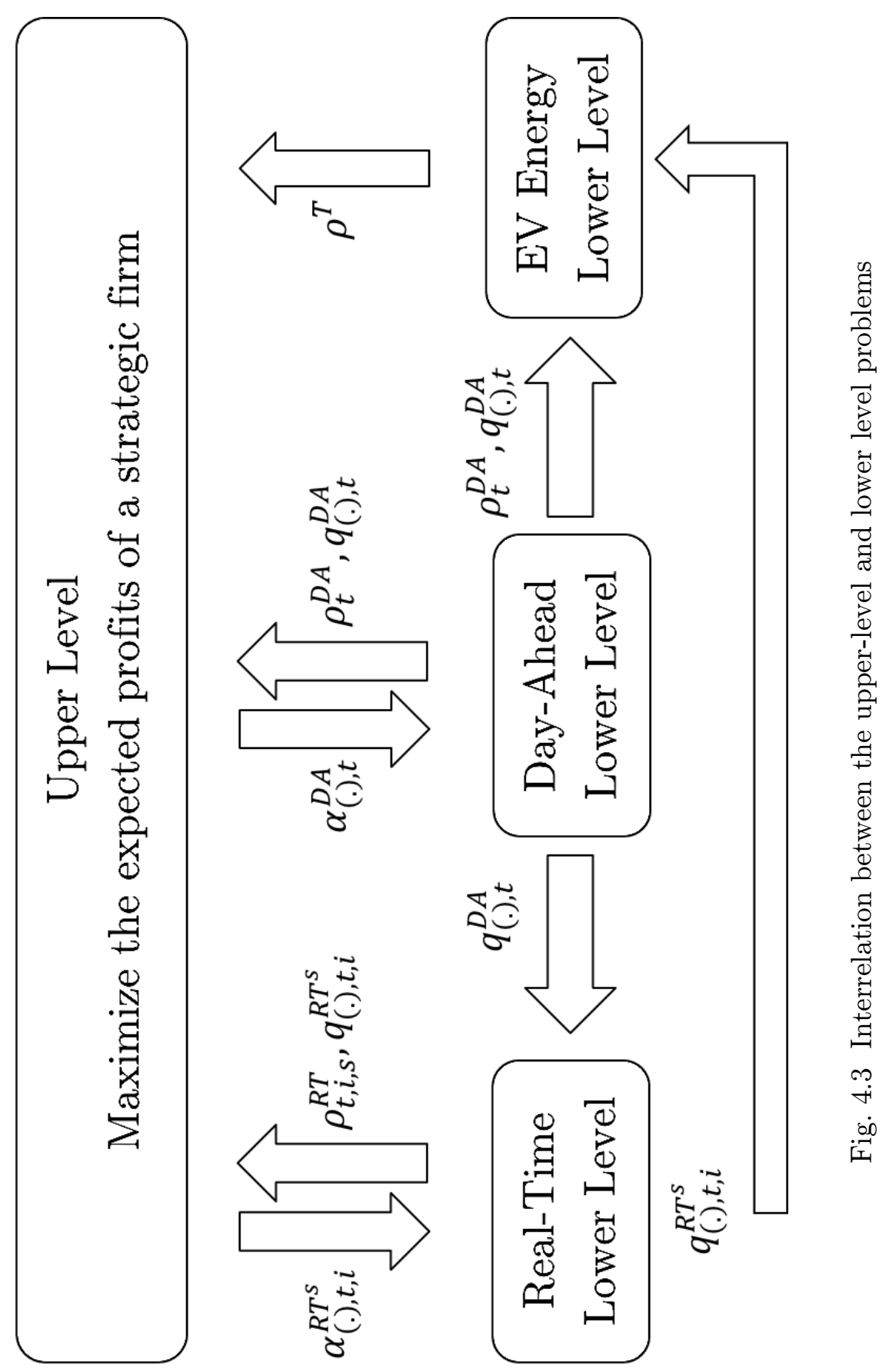




\subsubsection{Upper Level}

The Objective function of maximizing the expected profits of a strategic firm which owns both CGenCos and WGenCos and constitutes unidirectional V2G services is as follows [100]:

$$
\begin{aligned}
\underset{\Omega_{\text {prim }}, \Omega_{\text {dual }}}{\operatorname{Max}} \text { Profit }^{s}= & \\
& \sum_{s} \pi_{s} \cdot\left[P F_{s}^{\mathrm{CGenCo}}+P F_{s}^{\mathrm{WGenCo}}+P F_{s}^{\mathrm{Agg}}\right]
\end{aligned}
$$

The expected profit of CGenCos is revenue minus cost of generation in the day-ahead market, and revenue minus cost of regulation up/down in the real-time markets, given by (4.2):

$$
\begin{aligned}
P F_{s}^{\mathrm{CGenCo}}= & \sum_{t} \sum_{g} P_{g, t}^{D A} \cdot\left(\rho_{t}^{D A}-C_{g}\right) \\
& +\frac{1}{N_{I}} \sum_{i} \sum_{t} \sum_{g}\left(R_{g, t, i}^{u p^{s}}-R_{g, t, i}^{d o w n^{s}}\right)\left(\rho_{t, i, s}^{R T}-C_{g}\right)
\end{aligned}
$$

The expected profit of WGenCos is revenue of generation in the day-ahead market, and revenue minus cost of excess/shortage generation in the real-time markets, given by (4.3):

$$
\begin{aligned}
P F_{s}^{W G e n C o}= & \sum_{t} \sum_{w} P_{w, t}^{D A} \cdot \rho_{t}^{D A} \\
& -\frac{1}{N_{I}} \sum_{i} \sum_{t} \sum_{w}\left(P_{w, t}^{D A}+P_{w, t, i}^{C^{s}}-P_{w, t, i}^{R T^{s}}\right) \cdot \rho_{t, i, s}^{R T}
\end{aligned}
$$

Finally, the EV aggregator's revenue is obtained by selling ancillary services, as well as selling energy to its clients at the tariff $\left(\rho^{T}\right)$. The aggregator encourages EV owners to join in by offering an attractive price for charging which is low in comparison with petrol and energy prices. The EV aggregator's cost is associated 
with buying energy for EV charging. Hence, the EV aggregator's payoff is represented as:

$$
\begin{aligned}
P F_{s}^{A g g}= & -\sum_{t} \sum_{e v} P O P_{e v, t} \rho_{t}^{D A}-\rho^{T} \\
& +\frac{1}{N_{I}} \sum_{i} \sum_{t} \sum_{e v}\left(R_{e v, t, i}^{u p^{s}}-R_{e v, t, i}^{d o w n^{s}}\right)\left(\rho_{t, i, s}^{R T}-\rho^{T}\right)
\end{aligned}
$$

The primal variables $\Omega_{\text {prim }}$ of the upper-level problem include positive offering/bidding variables $\alpha_{g, t}^{D A}, \quad \alpha_{w, t}^{D A}, \beta_{e v, t}^{D A}, \alpha_{g, t, i}^{R T^{s}}, \alpha_{w, t, i}^{R T^{s}}, \alpha_{e v, t, i}^{R T^{s}}$ and variable sets $\Omega_{p r i m}^{D A}=\left\{\rho_{t}^{D A}, P_{g, t}^{D A}, P_{w, t}^{D A}, P O P_{e v, t}\right\}$, and $\Omega_{p r i m}^{R T}=\left\{\begin{array}{l}\rho_{t, i, s}^{R T}, R_{e v, t, i}^{u p^{s}}, R_{e v, t, i}^{d o w n^{s}} \\ R_{g, t, i}^{u p^{s}}, R_{g, t, i}^{d o w n^{s}}, P_{w, t, i}^{C^{s}}\end{array}\right\}$

\subsubsection{Day-Ahead Lower Level}

The day-ahead lower-level problem is formulated in this subsection. The dayahead market clearing is addressed with the aim of maximization of social welfare given by (4.5):

$$
\operatorname{Min}_{\left\{\Omega_{p r i m}^{D A}, \Omega_{d u a l}^{D A}\right\}}\left[\begin{array}{c}
\sum_{g, t} \alpha_{g, t}^{D A} P_{g, t}^{D A}+\sum_{w, t} \alpha_{w, t}^{D A} P_{w, t}^{D A}- \\
\sum_{d, t} B_{d, t}^{D A} L_{d, t}^{D A}-\sum_{e v, t} \beta_{e v, t}^{D A} P O P_{e v, t}
\end{array}\right]
$$

The primal variables of the lower-level problem (4.5) are those in set $\Omega_{\text {prim }}^{D A}$, and their dual variables in set $\Omega_{d u a l}^{D A}$ where dual variables are indicated following a colon at the constraints.

The energy balance in the day-ahead market is given by:

$$
\sum_{g} P_{g, t}^{D A}+\sum_{w} P_{w, t}^{D A}-\sum_{d} L_{d, t}^{D A}-\sum_{e v} P O P_{e v, t}=0 \quad: \rho_{t}^{D A}
$$

where dual variable $\rho_{t}^{D A}$ provides the day-ahead equilibrium price. 
Constraint for the EV POP is given by:

$$
0 \leq P O P_{e v, t} \leq P_{e v}^{\max } \quad: \mu_{e v, t}^{D A^{\max }}, \mu_{e v, t}^{D A^{\text {min }}} \quad \forall e v, t
$$

Constraints in (4.8) and (4.9) limit the scheduled power production of conventional and wind units, respectively.

$$
\begin{array}{lll}
0 \leq P_{g, t}^{D A} \leq P_{g}^{\max } & : \mu_{g, t}^{D A^{\max }}, \mu_{g, t}^{D A^{\text {min }}} & \forall g, t \\
0 \leq P_{w, t}^{D A} \leq P_{w}^{\max } & : \mu_{w, t}^{D A^{\max }}, \mu_{w, t}^{D A^{\text {min }}} & \forall w, t
\end{array}
$$

The day-ahead scheduled demand is limited in (4.10).

$$
0 \leq L_{d, t}^{D A} \leq L_{d}^{\max } \quad: \mu_{d, t}^{D A^{\max }}, \mu_{d, t}^{D A^{\min }} \quad \forall d, t
$$

\subsubsection{Real-Time Lower Level}

The real-time lower-level problem represents the real-time market clearing with the aim of maximization of social welfare for scenarios $s$ given by (4.11):

$$
\operatorname{Min}_{\left\{\Omega_{p r i m}^{R T}, \Omega_{d u a l}^{R T}\right\}}\left[\begin{array}{c}
\sum_{g, t, i}\left(R_{g, t, i}^{u p^{s}}-R_{g, t, i}^{d o w n^{s}}\right) \cdot \alpha_{g, t, i}^{R T^{s}}+ \\
\sum_{e v, t, i}\left(R_{e v, t, i}^{u p^{s}}-R_{e v, t, i}^{d o w n^{s}}\right) \cdot \alpha_{e v, t, i}^{R T^{s}}+ \\
\sum_{w, t, i}\left(P_{w, t, i}^{C^{s}} \cdot \alpha_{w, t, i}^{R T^{s}}\right)+\sum_{d, t, i} C_{d}^{L} L_{d, t, i}^{C^{s}}
\end{array}\right]
$$

The primal variables of the lower-level problem (4.11) are those in set $\Omega_{\text {prim }}^{R T}$, and their dual variables in set $\Omega_{d u a l}^{R T}$ where dual variables are shown at the corresponding constraints following a colon.

The energy balance in the real-time market is given by:

$$
\left.\sum_{w}\left(P_{w, t}^{D A}+P_{w, t, i}^{C^{s}}-P_{w, t, i}^{R T^{s}}\right)-\sum_{g}\left(R_{g, t, i}^{u p^{s}}-R_{g, t, i}^{\text {down }}\right)^{s}\right)-
$$




$$
\sum_{d} L_{d, t, i}^{C^{s}}-\sum_{e v}\left(R_{e v, t, i}^{u p^{s}}-R_{e v, t, i}^{d o w n^{s}}\right)=0 \quad: \rho_{t, i, s}^{R T} \quad \forall s, t, i
$$

where dual variable $\rho_{t, i, s}^{R T}$ provides the real-time equilibrium prices.

The capacity limits for regulation down $\left(R_{e v, t, i}^{d o w n}\right)$ to increase the EV charging rate, and regulation up $\left(R_{e v, t, i}^{u p^{s}}\right)$ to decrease the $\mathrm{EV}$ charging rate are given in (4.13)-(4.16)

$$
\begin{aligned}
& 0 \leq R_{e v, t, i}^{u p^{s}} \leq R_{e v}^{u p^{\max }} \quad: \mu_{e v, t, i, s}^{u p^{\max }}, \mu_{e v, t, i, s}^{u p^{\min }} \quad \forall e v, s, t, i \\
& R_{e v, t, i}^{u p^{s}} \leq P O P_{e v, t} \quad: \mu_{e v, t, i, s}^{u p} \quad \forall e v, s, t, i
\end{aligned}
$$

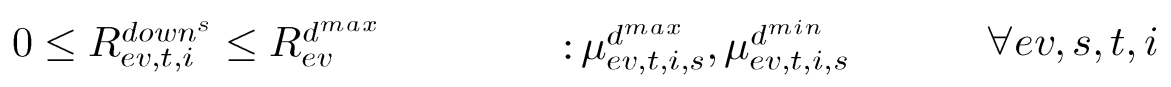

$$
\begin{aligned}
& P O P_{e v, t}+R_{e v, t, i}^{d o w n} \leq P_{e v}^{\max } \quad: \mu_{e v, t, i, s}^{\text {down }} \quad \forall e v, s, t, i
\end{aligned}
$$

Constraints (4.17) and (4.18) refer to the lower and upper bounds on the up and down regulations deployed from each dispatchable unit.

$$
\begin{array}{lll}
0 \leq R_{g, t, i}^{u p^{s}} \leq R_{g}^{u p^{\max }} & : \mu_{g, t, i, s}^{u p^{\max }}, \mu_{g, t, i, s}^{u p^{\min }} & \forall g, s, t, i \\
P_{g, t}^{D A}+R_{g, t, i}^{u p^{s}} \leq R_{g}^{u p^{\max }} & : \mu_{g, t, i, s}^{u p} & \forall g, s, t, i
\end{array}
$$

The constraints (4.19) and (4.20) guarantee that the power productions of conventional units are less than their capacities.

$$
\begin{array}{lll}
0 \leq R_{g, t, i}^{\text {down }^{s}} \leq R_{g}^{d^{\text {max }}} & : \mu_{g, t, i, s}^{d^{\text {max }}}, \mu_{g, t, i, s}^{d^{\text {min }}} & \forall g, s, t, i \\
R_{g, t, i}^{\text {down }} \leq P_{g, t}^{D A} & : \mu_{g, t, i, s}^{\text {down }} & \forall g, s, t, i
\end{array}
$$

The constraints (4.21) and (4.22) limit the minimum and maximum for the wind power and load demand curtailment. 


$$
\begin{array}{lll}
0 \leq P_{w, t, i}^{C^{s}} \leq P_{w, t, i}^{R T^{s}} & : \mu_{w, t, i, s}^{c^{\max }}, \mu_{w, t, i, s}^{c^{\min }} & \forall w, s, t, i \\
0 \leq L_{d, t, i}^{C^{s}} \leq L_{d}^{\max } & : \mu_{d, t, i, s}^{c^{\max }}, \mu_{d, t, i, s}^{c^{\min }} & \forall d, s, t, i
\end{array}
$$

\subsubsection{EV Energy Lower Level}

The EV Energy lower-level problem aims to maximize EV energy capacity given in

$$
\underset{\left\{E_{e v, t}^{D A}, \Omega_{d u a l}^{E V}\right\}}{\operatorname{Min}}\left[-\sum_{e v, t} \rho_{t}^{D A} E_{e v, t}^{D A}\right]
$$

The energy balance equation for the EV fleet is given in

$$
\begin{aligned}
& \sum_{e v} E_{e v, t, i}^{s}-\sum_{e v} E_{e v, t, i-1}^{s}-\left(1-N_{e v, t, i}^{s}\right) . D_{e v, t, i}^{s} \\
& -\frac{1}{N_{I}} \sum_{e v}\left(P O P_{e v, t}-R_{e v, t, i}^{u p^{s}}+R_{e v, t, i}^{d o w n^{s}}\right)=0 \quad: \rho^{T}, \quad \forall s, t, i \\
& E_{e v, t}^{D A}=\frac{1}{N_{I}} \sum_{s} \sum_{i} E_{e v, t, i}^{s}
\end{aligned}
$$

The EV energy constraint is presented in

$$
\begin{aligned}
& \frac{1}{N_{I}} \sum_{s} \sum_{i} \sum_{e v} E_{e v, t}^{M i n} \cdot N_{e v, t, i}^{s} . S O C_{E V}^{\min } \\
& \leq E_{e v, t}^{D A} \\
& \leq \frac{1}{N_{I}} \sum_{s} \sum_{i} \sum_{e v} E_{e v, t}^{M a x} \cdot N_{e v, t, i}^{s} \cdot S O C_{E V}^{\max }: \mu_{e v, t}^{E E V^{m a x}}, \mu_{e v, t}^{E E V^{m i n}} \forall e v, s, t,
\end{aligned}
$$

\subsubsection{Mathematical Program with Equilibrium Constraints}

The stochastic bilevel model including multi-optimization problems transforms into a single optimization problem as a single-level stochastic MPEC [71]. The 
lower problems are continuous linear and can be replaced by their KKT conditions.

\subsubsection{KKT Conditions Corresponding to the Day-ahead Lower Level}

The corresponding Lagrangian function $\mathcal{L}^{D A}$ of a day-ahead clearing problem is defined as follows:

$$
\begin{aligned}
& \partial \mathcal{L}^{D A}=\sum_{g, t} \alpha_{g, t}^{D A} P_{g, t}^{D A}+\sum_{w, t} \alpha_{w, t}^{D A} P_{w, t}^{D A} \\
& -\sum_{d, t} B_{d, t}^{D A} L_{d, t}^{D A}-\sum_{e v, t} \beta_{e v, t}^{D A} P O P_{e v, t} \\
& +\sum_{t} \rho_{t}^{D A}\left(\sum_{g} P_{g, t}^{D A}+\sum_{w} P_{w, t}^{D A}-\sum_{d} L_{d, t}^{D A}-\sum_{e v} P O P_{e v, t}\right) \\
& +\sum_{e v, t} \mu_{e v, t}^{D A^{\max }}\left(P_{e v}^{\max }-P O P_{e v, t}\right)-\sum_{e v, t} \mu_{e v, t}^{D A^{m i n}} P O P_{e v, t} \\
& +\sum_{g, t} \mu_{g, t}^{D A^{\max }}\left(P_{g}^{\max }-P_{g, t}^{D A}\right)-\sum_{g, t} \mu_{g, t}^{D A^{m i n}} P_{g, t}^{D A} \\
& +\sum_{w, t} \mu_{w, t}^{D A^{\max }}\left(P_{w}^{\max }-P_{w, t}^{D A}\right)-\sum_{w, t} \mu_{w, t}^{D A^{m i n}} P_{w, t}^{D A} \\
& +\sum_{d, t} \mu_{d, t}^{D A^{\max }}\left(L_{d}^{\max }-L_{d, t}^{D A}\right)-\sum_{d, t} \mu_{d, t}^{D A^{m i n}} L_{d, t}^{D A}
\end{aligned}
$$

The first-order KKT conditions associated with the day-ahead lower level according to Lagrangian function (4.27) are derived as given by:

$$
\frac{\partial \mathcal{L}^{D A}}{\partial P O P_{e v, t}}=-\beta_{e v, t}^{D A}+\rho_{t}^{D A}+\mu_{e v, t}^{D A^{\text {max }}}-\mu_{e v, t}^{D A^{m i n}}=0 \quad \forall e v, t
$$




$$
\begin{aligned}
& \frac{\partial \mathcal{L}^{D A}}{\partial P_{g, t}^{D A}}=+\alpha_{g, t}^{D A}-\rho_{t}^{D A}+\mu_{g, t}^{D A^{\max }}-\mu_{g, t}^{D A^{\text {min }}}=0 \quad \forall g, t \\
& \frac{\partial \mathcal{L}^{D A}}{\partial P_{w, t}^{D A}}=+\alpha_{w, t}^{D A}-\rho_{t}^{D A}+\mu_{w, t}^{D A^{\text {max }}}-\mu_{w, t}^{D A^{\text {min }}}=0 \quad \forall w, t \\
& \frac{\partial \mathcal{L}^{D A}}{\partial L_{d, t}^{D A}}=-B_{d, t}^{D A}+\rho_{t}^{D A}+\mu_{d, t}^{D A^{\max }}-\mu_{d, t}^{D A^{\text {min }}}=0 \quad \forall d, t \\
& 0 \leq P_{e v}^{\max }-P O P_{e v, t} \perp \mu_{e v, t}^{D A^{\max }} \geq 0 \quad \forall e v, t \\
& 0 \leq P_{g}^{\max }-P_{g, t}^{D A} \perp \mu_{g, t}^{D A^{\max }} \geq 0 \quad \forall g, t \\
& 0 \leq P_{w}^{\max }-P_{w, t}^{D A} \perp \mu_{w, t}^{D A^{\max }} \geq 0 \quad \forall w, t \\
& 0 \leq L_{d}^{\max }-L_{d, t}^{D A} \perp \mu_{d, t}^{D A^{\max }} \geq 0 \quad \forall d, t \\
& 0 \leq P O P_{e v, t} \perp \mu_{e v, t}^{D A^{m i n}} \geq 0 \quad \forall e v, t \\
& 0 \leq P_{g, t}^{D A} \perp \mu_{g, t}^{D A^{m i n}} \geq 0 \quad \forall g, t \\
& 0 \leq P_{w, t}^{D A} \perp \mu_{w, t}^{D A^{m i n}} \geq 0 \quad \forall w, t \\
& 0 \leq L_{d, t}^{D A} \perp \mu_{d, t}^{D A^{m i n}} \geq 0 \quad \forall d, t
\end{aligned}
$$

where $\perp$ denotes the inner product of two vectors equal to zero [57].

\subsubsection{KKT Conditions Corresponding to the Real-time Lower Level}

The corresponding Lagrangian function $\mathcal{L}^{R T}$ of the real-time clearing problems is defined as follows: 


$$
\begin{aligned}
& \mathcal{L}^{R T}=\sum_{w, t, i}\left(P_{w, t, i}^{C^{s}} \cdot \alpha_{w, t, i}^{R T^{s}}\right)+\sum_{d, t, i} C_{d}^{L} L_{d, t, i}^{C^{s}}+\sum_{e v, t, i}\left(R_{e v, t, i}^{u p^{s}}-R_{e v, t, i}^{d o w n^{s}}\right) \cdot \alpha_{g, t, i}^{R T^{s}} \\
& +\sum_{g, t, i}\left(R_{g, t, i}^{u p^{s}}-R_{g, t, i}^{d o w n^{s}}\right) \cdot \alpha_{g, t, i}^{R T^{s}} \\
& +\sum_{t} \rho_{t, i, s}^{R T}\left(\sum_{w}\left(P_{w, t}^{D A}+P_{w, t, i}^{C^{s}}-P_{w, t, i}^{R T^{s}}\right)\right. \\
& -\sum_{g}\left(R_{g, t, i}^{u p^{s}}-R_{g, t, i}^{d o w n^{s}}\right)-\sum_{d} L_{d, t, i}^{C^{s}} \\
& \left.-\sum_{e v}\left(R_{e v, t, i}^{u p^{s}}-R_{e v, t, i}^{d o w n^{s}}\right)\right) \\
& +\sum_{g, t, i} \mu_{g, t, i, s}^{u p^{\max }}\left(R_{g}^{u p^{\max }}-R_{g, t, i}^{u p^{s}}\right)-\sum_{g, t, i} \mu_{g, t, i, s}^{u p^{\min }} R_{g, t, i}^{u p^{s}} \\
& +\sum_{g, t, i} \mu_{e v, t, i, s}^{u p}\left(R_{g}^{u p^{\max }}-P_{g, t}^{D A}-R_{g, t, i}^{u p^{s}}\right) \\
& +\sum_{g, t, i} \mu_{g, t, i, s}^{d^{\max }}\left(R_{g}^{d^{\max }}-R_{g, t, i}^{d o w n^{s}}\right)-\sum_{g, t, i} \mu_{g, t, i, s}^{d^{\text {min }}} R_{g, t, i}^{\text {down }} \\
& +\sum_{g, t, i} \mu_{g, s, R T}^{\text {down }}\left(P_{g, t}^{D A}-R_{g, t, i}^{\text {down }}\right) \\
& +\sum_{e v, t, i} \mu_{e v, t, i, s}^{u p^{\max }}\left(R_{e v}^{u p^{\max }}-R_{e v, t, i}^{u p^{s}}\right)-\sum_{e v, t, i} \mu_{e v, t, i, s}^{u p^{\min }} R_{e v, t, i}^{u p^{s}} \\
& +\sum_{e v, t, i} \mu_{e v, t, i, s}^{u p}\left(P O P_{e v, t}-R_{e v, t, i}^{u p^{s}}\right) \\
& +\sum_{e v, t, i}\left(R_{e v}^{d^{\max }}-R_{e v, t, i}^{d o w n^{s}}\right) \mu_{e v, t, i, s}^{d^{\max }}-\sum_{e v, t, i} \mu_{e v, t, i, s}^{d^{\text {min }}} R_{e v}^{d^{\text {max }}} \\
& +\sum_{e v, t, i} \mu_{e v, t, i, s}^{d}\left(P_{e v}^{\max }-P O P_{e v, t}-R_{e v, t, i}^{d o w n^{s}}\right) \\
& +\sum_{d, t, i} \mu_{d, t, i, s}^{c^{\max }}\left(L_{d}^{\max }-L_{d, t, i}^{C^{s}}\right)-\sum_{d, t, i} \mu_{d, t, i, s}^{c^{\min }} L_{d, t, i}^{C^{s}} \\
& +\sum_{w t, i} \mu_{w, t, i, s}^{c^{\max }}\left(P_{w, t, i}^{R T^{s}}-P_{w, t, i}^{C^{s}}\right)-\sum_{w t, i} \mu_{w, t, i, s}^{c^{m i n}} P_{w, t, i}^{C^{s}}
\end{aligned}
$$

The first-order KKT conditions associated with the real-time lower level according to Lagrangian function (4.28) are derived as given by" 


$$
\begin{aligned}
& \frac{\partial \mathcal{L}^{R T}}{\partial R_{e v, t, i}^{u p^{s}}}=\mu_{e v, t, i, s}^{u p^{\max }}-\mu_{e v, t, i, s}^{u p^{m i n}}+\mu_{e v, t, i, s}^{u p}+\alpha_{e v, t, i}^{R T^{s}}-\rho_{t, i, s}^{R T}=0 \quad \forall e v, s, t, i \\
& \frac{\partial \mathcal{L}^{R T}}{\partial R_{e v, t, i}^{d^{s}}}=\mu_{e v, t, i, s}^{d^{\text {max }}}-\mu_{e v, t, i, s}^{d^{m i n}}+\mu_{e v, t, i, s}^{d o w n}-\alpha_{e v, t, i}^{R T^{s}}+\rho_{t, i, s}^{R T}=0 \quad \forall e v, s, t, i \\
& \frac{\partial \mathcal{L}^{R T}}{\partial R_{g, t, i}^{u p^{s}}}=\mu_{g, t, i, s}^{u p^{\max }}-\mu_{g, t, i, s}^{u p^{m i n}}+\mu_{g, t, i, s}^{u p}+\alpha_{g, t, i}^{R T^{s}}-\rho_{t, i, s}^{R T}=0 \quad \forall g, s, t, i \\
& \frac{\partial \mathcal{L}^{R T}}{\partial R_{g, t, i}^{d^{s}}}=\mu_{g, t, i, s}^{d^{m a x}}-\mu_{g, t, i, s}^{d^{m i n}}+\mu_{g, s, R T}^{d o w n}-\alpha_{g, t, i}^{R T^{s}}+\rho_{t, i, s}^{R T}=0 \quad \forall g, s, t, i \\
& \frac{\partial \mathcal{L}^{R T}}{\partial P_{w, t, i}^{C^{s}}}=\mu_{w, t, i, s}^{c^{\max }}-\mu_{w, t, i, s}^{c^{m i n}}+\alpha_{w, t, i}^{R T^{s}}+\rho_{t, i, s}^{R T}=0 \quad \forall w, s, t, i \\
& \frac{\partial \mathcal{L}^{R T}}{\partial L_{d, t, i}^{C^{s}}}=-\rho_{t, i, s}^{R T}+\mu_{d, t, i, s}^{c^{m a x}}-\mu_{d, t, i, s}^{c^{m i n}}+C_{d, t}^{L}=0 \quad \forall d, s, t, i \\
& 0 \leq R_{e v}^{u p^{\max }}-R_{e v, t, i}^{u p^{s}} \perp \mu_{e v, t, i, s}^{u p^{\max }} \geq 0 \\
& \forall e v, s, t, i \quad(4.28 \mathrm{~g}) \\
& 0 \leq P O P_{e v, t}-R_{e v, t, i}^{u p^{s}} \perp \mu_{e v, t, i, s}^{u p} \geq 0 \\
& \forall e v, s, t, i \quad(4.28 \mathrm{~h}) \\
& 0 \leq R_{e v}^{d^{\text {max }}}-R_{e v, t, i}^{d o w n^{s}} \perp \mu_{e v, t, i, s}^{d^{\max }} \geq 0 \quad \forall e v, s, t, i \\
& 0 \leq P_{e v}^{\max }-P O P_{e v, t}-R_{e v, t, i}^{d o w n^{s}} \perp \mu_{e v, s, R T}^{d o w n} \geq 0 \quad \forall e v, s, t, i \\
& 0 \leq R_{g}^{u p^{\max }}-R_{g, t, i}^{u p^{s}} \perp \mu_{g, t, i, s}^{u p^{\max }} \geq 0 \\
& \forall g, s, t, i \\
& 0 \leq R_{g}^{u p^{\max }}-P_{g, t}^{D A}-R_{g, t, i}^{u p^{s}} \perp \mu_{g, t, i, s}^{u p} \geq 0 \quad \forall g, s, t, i \\
& 0 \leq R_{g}^{d^{\text {max }}}-R_{g, t, i}^{\text {down }} n^{s} \perp \mu_{g, t, i, s}^{d^{\max }} \geq 0 \\
& \forall g, s, t, i \quad(4.28 \mathrm{~m}) \\
& 0 \leq P_{g, t}^{D A}-R_{g, t, i}^{d o w n^{s}} \perp \mu_{g, s, R T}^{\text {down }} \geq 0 \\
& \forall g, s, t, i \\
& 0 \leq P_{w, t, i}^{R T^{s}}-P_{w, t, i}^{C^{s}} \perp \mu_{w, t, i, s}^{c^{\max } \geq 0} \quad \forall w, s, t, i \\
& 0 \leq L_{d}^{\max }-L_{d, t, i}^{C^{s}} \perp \mu_{d, t, i, s}^{c^{\max }} \geq 0 \quad \forall d, s, t, i
\end{aligned}
$$




$$
\begin{aligned}
& 0 \leq R_{e v, t, i}^{u p^{s}} \perp \mu_{e v, t, i, s}^{u p^{m i n}} \geq 0 \\
& \forall e v, s, t, i \quad(4.28 \mathrm{q}) \\
& 0 \leq R_{e v, t, i}^{d o w n^{s}} \perp \mu_{e v, t, i, s}^{d^{m i n}} \geq 0 \\
& \forall e v, s, t, i \\
& 0 \leq R_{g, t, i}^{u p^{s}} \perp \mu_{g, t, i, s}^{u p^{m i n}} \geq 0 \\
& \forall g, s, t, i \\
& 0 \leq R_{g, t, i}^{d o w n^{s}} \perp \mu_{g, t, i, s}^{d^{m i n}} \geq 0 \\
& \forall g, s, t, i \\
& 0 \leq P_{w, t, i}^{C^{s}} \perp \mu_{w, t, i, s}^{c^{m i n}} \geq 0 \\
& \forall w, s, t, i \\
& 0 \leq L_{d, t, i}^{C^{s}} \perp \mu_{d, t, i, s}^{c^{m i n}} \geq 0 \\
& \forall d, s, t, i
\end{aligned}
$$

\subsubsection{KKT Conditions Corresponding to the EV Lower Level}

The corresponding Lagrangian function $\mathcal{L}^{E V}$ of the EV lower-level problem is defined as follows:

$$
\begin{aligned}
& \mathcal{L}^{E V}=-\sum_{e v, t} \rho_{t}^{D A} E_{e v, t}^{D A} \\
& +\sum_{t} \rho^{T}\left(\frac { 1 } { N _ { I } } \sum _ { s } \sum _ { i } \left[\sum_{e v} E_{e v, t, i}^{s}\right.\right. \\
& -\sum_{e v} E_{e v, t, i-1}^{s}-\left(1-N_{e v, t, i}^{s}\right) \cdot D_{e v, t, i}^{s} \\
& \left.\left.-\frac{1}{N_{I}} \sum_{e v}\left(P O P_{e v, t}-R_{e v, t, i}^{u p^{s}}+R_{e v, t, i}^{d o w n^{s}}\right)\right]\right) \\
& +\sum_{t} \mu_{e v, t}^{E E V^{m a x}}\left(E_{e v, t}^{D A}-\frac{1}{N_{I}} \sum_{s} \sum_{i} \sum_{e v} E_{e v, t}^{M a x} \cdot N_{e v, t, i}^{s} \cdot S O C_{E V}^{\max }\right) \\
& -\sum_{t} \mu_{e v, t}^{E E V^{m i n}}\left(\frac{1}{N_{I}} \sum_{s} \sum_{i} \sum_{e v} E_{e v, t}^{M i n} \cdot N_{e v, t, i}^{s} \cdot S O C_{E V}^{\min }-E_{e v, t}^{D A}\right)
\end{aligned}
$$

The first-order KKT conditions associated with the real-time lower level according to Lagrangian function (4.29) are derived as given by 


$$
\begin{array}{ll}
\frac{\partial \mathcal{L}^{E V}}{\partial E_{e v, t}^{D A}}=\rho^{T}-\rho_{t}^{D A}+\mu_{e v, t}^{E E V^{m a x}}-\mu_{e v, t}^{E E V^{m i n}}=0 & \forall e v, t \\
0 \leq E_{e v, t}^{D A}-\frac{1}{N_{I}} \sum_{s} \sum_{i} \sum_{e v} E_{e v, t}^{M a x} \cdot N_{e v, t, i}^{s} . S O C_{E V}^{m a x} \perp \mu_{e v, t}^{E E V^{m a x}} \geq 0 & \forall e v, t \\
0 \leq \frac{1}{N_{I}} \sum_{s} \sum_{i} \sum_{e v} E_{e v, t}^{M i n} \cdot N_{e v, t, i}^{s} . S O C_{E V}^{m i n}-E_{e v, t}^{D A} \perp \mu_{e v, t}^{E E V^{m i n}} \geq 0 & \forall e v, t
\end{array}
$$

\subsubsection{Strong Duality Theorem Corresponding to the Day-ahead} Lower Level

Equation (4.30) enforces the strong duality equality associated with the dayahead objective function.

$$
\begin{aligned}
\sum_{g, t} \alpha_{g, t}^{D A} P_{g, t}^{D A} & +\sum_{w, t} \alpha_{w, t}^{D A} P_{w, t}^{D A}-\sum_{d, t} B_{d, t}^{D A} L_{d, t}^{D A}-\sum_{e v, t} \beta_{e v, t}^{D A} P O P_{e v, t} \\
& +\sum_{e v, t} \mu_{e v, t}^{D A^{\max }} P_{e v}^{\max }+\sum_{g, t} \mu_{g, t}^{D A^{\max }}\left(P_{g}^{\max }\right) \\
& +\sum_{w, t} \mu_{w, t}^{D A^{\max }}\left(P_{w}^{\max }\right)+\sum_{d, t} \mu_{d, t}^{D A^{\max }} L_{d}^{\max }=0
\end{aligned}
$$

\subsubsection{Strong Duality Theorem Corresponding to the Real-time Lower Level}

Equation (4.31) enforces the strong duality equality associated with the dayahead objective function. 


$$
\begin{aligned}
& \sum_{w, t, i}\left(P_{w, t, i}^{C^{s}} \cdot \alpha_{w, t, i}^{R T^{s}}\right)+\sum_{d, t, i} C_{d}^{L} L_{d, t, i}^{C^{s}}+\sum_{e v, t, i}\left(R_{e v, t, i}^{u p^{s}}-R_{e v, t, i}^{d o w n^{s}}\right) \cdot \alpha_{g, t, i}^{R T^{s}} \\
& +\sum_{g, t, i}\left(R_{g, t, i}^{u p^{s}}-R_{g, t, i}^{d o w n^{s}}\right) \cdot \alpha_{g, t, i}^{R T^{s}}+\sum_{d, t, i} \mu_{d, t, i, s}^{c^{\max }} L_{d}^{\max } \\
& +\sum_{g, t, i} \mu_{g, t, i, s}^{u p^{\max }}\left(R_{g}^{u p^{\max }}\right)+\sum_{g, t, i} \mu_{e v, t, i, s}^{u p}\left(R_{g}^{u p^{\max }}-P_{g, t}^{D A}\right) \\
& +\sum_{e v, t, i} \mu_{e v, t, i, s}^{u p^{\max }} R_{e v}^{u p^{\max }}+\sum_{e v, t, i} \mu_{e v, t, i, s}^{u p}\left(P O P_{e v, t}\right) \\
& +\sum_{w t, i} \mu_{w, t, i, s}^{c^{\max }}\left(P_{w, t, i}^{R T^{s}}\right)+\sum_{e v, t, i} \mu_{e v, t, i, s}^{d}\left(P_{e v}^{\max }-P O P_{e v, t}\right) \\
& +\sum_{g, t, i} \mu_{g, t, i, s}^{d^{\max }}\left(R_{g}^{d^{\max }}\right)+\sum_{t} \rho_{t, i, s}^{R T}\left(\sum_{w}\left(P_{w, t}^{D A}-P_{w, t, i}^{R T^{s}}\right)\right) \\
& +\sum_{g, t, i} \mu_{g, s, R T}^{\text {down }}\left(P_{g, t}^{D A}\right)+\sum_{e v, t, i} R_{e v}^{d^{\max }} \mu_{e v, t, i, s}^{d^{\max }}=0
\end{aligned}
$$

4.3.2.6 Strong Duality Theorem Corresponding to the EV Lower Level

Equation (4.32) enforces the strong duality equality associated with EV lower problem.

$$
\begin{aligned}
& -\sum_{e v, t} \rho_{t}^{D A} E_{e v, t}^{D A} \\
& +\sum_{t} \rho^{T}\left(\frac { 1 } { N _ { I } } \sum _ { s } \sum _ { i } \left[-\sum_{e v} E_{e v, t, i-1}^{s}-\left(1-N_{e v, t, i}^{s}\right) \cdot D_{e v, t, i}^{s}\right.\right. \\
& \left.\left.-\frac{1}{N_{I}} \sum_{e v}\left(P O P_{e v, t}-R_{e v, t, i}^{u p^{s}}+R_{e v, t, i}^{d o w n n^{s}}\right)\right]\right) \\
& +\sum_{t} \mu_{e v, t}^{E E V^{\max }}\left(\frac{1}{N_{I}} \sum_{s} \sum_{i} \sum_{e v} E_{e v, t}^{M a x} \cdot N_{e v, t, i}^{s} \cdot S O C_{E V}^{\max }\right) \\
& -\sum_{t} \mu_{e v, t}^{E E V^{\min }}\left(\frac{1}{N_{I}} \sum_{s} \sum_{i} \sum_{e v} E_{e v, t}^{M i n} \cdot N_{e v, t, i}^{s} \cdot S O C_{E V}^{\min }\right)=0
\end{aligned}
$$




\subsubsection{Mixed-Integer Linear Programming}

The MPEC are converted to a MILP by the linearizing of two nonlinearities including complementarity conditions and nonlinear terms as follows.

\subsubsection{Linearization of Complementarity Conditions}

The complementarity conditions in the form of $0 \leq P \perp \mu \geq 0$ can be linearized by

$$
P \geq 0, \mu \geq 0, \mu \leq b . M_{1}, P \leq 1-b . M_{2}
$$

where $b$ is an auxiliary binary variable, and $M_{1}$ and $M_{2}$ are large enough constants. Note that the values of $M_{1}$ and $M_{2}$ are selected by trial-and error approach as used in [21].

1) Mixed-integer linear equivalents of the complementarity conditions in section 4.3.2.1 from equation $(4.27 \mathrm{e})$ to $(4.271)$ :

$$
\begin{array}{ll}
\mu_{e v, t}^{D A^{\max }} \geq 0, P_{e v}^{\max }-P O P_{e v, t} \geq 0 & \forall e v, t \\
P_{e v}^{\max }-P O P_{e v, t} \leq b_{e v, t}^{D A^{\text {max }}} X_{E}^{D A} & \forall e v, t \\
\mu_{e v, t}^{D A^{\max }} \leq\left(1-b_{e v, t}^{D A^{\text {max }}}\right) Y_{E}^{D A} & \forall e v, t \\
\mu_{g, t}^{D A^{\max }} \geq 0, P_{g}^{\max }-P_{g, t}^{D A} \geq 0 & \forall g, t \\
P_{g}^{\max }-P_{g, t}^{D A} \leq b_{g, t}^{D A^{\max }} X_{G}^{D A} & \forall g, t \\
\mu_{g, t}^{D A^{\max }} \leq\left(1-b_{g, t}^{D A^{\max }}\right) Y_{G}^{D A} & \forall g, t \\
\mu_{w, t}^{D A^{\max }} \geq 0, P_{w}^{\max }-P_{w, t}^{D A} \geq 0 & \forall w, t \\
P_{w}^{\max }-P_{w, t}^{D A} \leq b_{w, t}^{D A^{\max }} X_{W}^{D A} & \forall w, t
\end{array}
$$




$$
\begin{aligned}
& \mu_{w, t}^{D A^{\max }} \leq\left(1-b_{w, t}^{D A^{\max }}\right) Y_{W}^{D A} \quad \forall w, t \\
& \mu_{d, t}^{D A^{\max }} \geq 0, L_{d}^{\max }-L_{d, t}^{D A} \geq 0 \quad \forall d, t \\
& L_{d}^{\max }-L_{d, t}^{D A} \leq b_{d, t}^{D A^{\max }} X_{L}^{D A} \quad \forall d, t \\
& \mu_{d, t}^{D A^{\max }} \leq\left(1-b_{d, t}^{D A^{\max }}\right) Y_{L}^{D A} \quad \forall d, t \\
& \mu_{e v, t}^{D A^{\text {min }}} \geq 0, P O P_{e v, t} \geq 0 \quad \forall e v, t \\
& P O P_{e v, t} \leq b_{e v, t}^{D A^{m i n}} X_{E}^{D A} \quad \forall e v, t \\
& \mu_{e v, t}^{D A^{\max }} \leq\left(1-b_{e v, t}^{D A^{\text {min }}}\right) Y_{E}^{D A} \quad \forall e v, t \\
& \mu_{g, t}^{D A^{m i n}} \geq 0, P_{g, t}^{D A} \geq 0 \quad \forall g, t \\
& P_{g, t}^{D A} \leq b_{g, t}^{D A^{m i n}} X_{G}^{D A} \quad \forall g, t \\
& \mu_{g, t}^{D A^{m i n}} \leq\left(1-b_{g, t}^{D A^{m i n}}\right) Y_{G}^{D A} \quad \forall g, t \\
& \mu_{w, t}^{D A^{m i n}} \geq 0, P_{w, t}^{D A} \geq 0 \quad \forall w, t \\
& P_{w, t}^{D A} \leq b_{w, t}^{D A^{m i n}} X_{W}^{D A} \quad \forall w, t \\
& \mu_{w, t}^{D A^{m i n}} \leq\left(1-b_{w, t}^{D A^{m i n}}\right) Y_{W}^{D A} \quad \forall w, t \\
& \mu_{d, t}^{D A^{\min }} \geq 0, L_{d, t}^{D A} \geq 0 \quad \forall d, t \\
& L_{d, t}^{D A} \leq b_{d, t}^{D A^{m i n}} X_{L}^{D A} \quad \forall d, t \\
& \mu_{d, t}^{D A^{m i n}} \leq\left(1-b_{d, t}^{D A^{m i n}}\right) Y_{L}^{D A} \quad \forall d, t
\end{aligned}
$$

where $X_{E}^{D A}, X_{G}^{D A}, X_{W}^{D A}, X_{L}^{D A}, Y_{E}^{D A}, Y_{G}^{D A}, Y_{W}^{D A}, Y_{L}^{D A}$ are large enough positive constants and $b_{(.), t}^{D A^{(\cdot)}}$ are binary variables.

2) Mixed-integer linear equivalents of the complementarity conditions in section 4.3.2.2 from equation $(4.28 \mathrm{~g})$ to $(4.28 \mathrm{y})$ : 


$$
\begin{aligned}
& \mu_{e v, t, i, s}^{u p^{\max }} \geq 0, R_{e v}^{u p^{\max }}-R_{e v, t, i}^{u p^{s}} \geq 0 \quad \forall e v, s, t, i \\
& R_{e v}^{u p^{\max }}-R_{e v, t, i}^{u p^{s}} \leq b_{e v, t, i, s}^{u p^{\max }} X_{E}^{R T} \quad \forall e v, s, t, i \\
& \mu_{e v, t, i, s}^{u p^{\max }} \leq\left(1-b_{e v, t, i, s}^{u p^{\max }}\right) Y_{E}^{R T} \quad \forall e v, s, t, i \\
& \mu_{e v, t, i, s}^{u p} \geq 0, P O P_{e v, t}-R_{e v, t, i}^{u p^{s}} \geq 0 \quad \forall e v, s, t, i \\
& P O P_{e v, t}-R_{e v, t, i}^{u p^{s}} \leq b_{e v, t, i, s}^{u p} X_{E}^{R T} \quad \forall e v, s, t, i \\
& \mu_{e v, t, i, s}^{u p} \leq\left(1-b_{e v, t, i, s}^{u p}\right) Y_{E}^{R T} \\
& \forall e v, s, t, i
\end{aligned}
$$

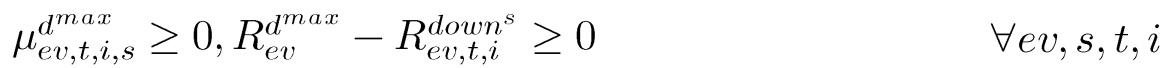

$$
\begin{aligned}
& R_{e v}^{d^{\text {max }}}-R_{e v, t, i}^{\text {down }} \leq b_{e v, t, i, s}^{d^{\text {max }}} X_{E}^{R T} \quad \forall e v, s, t, i
\end{aligned}
$$

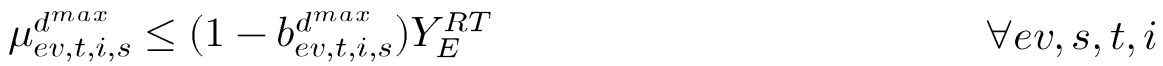

$$
\begin{aligned}
& \mu_{e v, s, R T}^{d o w n} \geq 0, P_{e v}^{\max }-P O P_{e v, t}-R_{e v, t, i}^{d o w n^{s}} \geq 0 \quad \forall e v, s, t, i \\
& P_{e v}^{m a x}-P O P_{e v, t}-R_{e v, t, i}^{d o w n} \leq b_{e v, s, R T}^{d o w n} X_{E}^{R T} \quad \forall e v, s, t, i \\
& \mu_{e v, s, R T}^{\text {down }} \leq\left(1-b_{e v, s, R T}^{\text {down }}\right) Y_{E}^{R T} \quad \forall e v, s, t, i \\
& \mu_{g, t, i, s}^{u p^{\max }} \geq 0, R_{g}^{u p^{\max }}-R_{g, t, i}^{u p^{s}} \geq 0 \quad \forall g, s, t, i \\
& R_{g}^{u p^{\max }}-R_{g, t, i}^{u p^{s}} \leq b_{g, t, i, s}^{u p^{\max }} X_{G}^{R T} \quad \forall g, s, t, i \\
& \mu_{g, t, i, s}^{u p^{\max }} \leq\left(1-b_{g, t, i, s}^{u p^{\max }}\right) Y_{G}^{R T} \quad \forall g, s, t, i \\
& \mu_{g, t, i, s}^{u p} \geq 0, R_{g}^{u p^{\max }}-P_{g, t}^{D A}-R_{g, t, i}^{u p^{s}} \geq 0 \quad \forall g, s, t, i \\
& R_{g}^{u p^{\max }}-P_{g, t}^{D A}-R_{g, t, i}^{u p^{s}} \leq b_{g, t, i, s}^{u p} X_{G}^{R T} \quad \forall g, s, t, i \\
& \mu_{e v, t, i, s}^{u p} \leq\left(1-b_{g, t, i, s}^{u p}\right) Y_{G}^{R T} \quad \forall g, s, t, i \\
& \mu_{g, t, i, s}^{d^{\max }} \geq 0, R_{g}^{d^{\max }}-R_{g, t, i}^{\text {down }} n^{s} \geq 0 \quad \forall g, s, t, i
\end{aligned}
$$




$$
\begin{aligned}
& R_{g}^{d^{\text {max }}}-R_{g, t, i}^{\text {down }} \text { s }^{s} \leq b_{g, t, i, s}^{d^{\text {max }}} X_{G}^{R T} \quad \forall g, s, t, i \\
& \mu_{g, t, i, s}^{d_{\max }^{\max }} \leq\left(1-b_{g, t, i, s}^{d^{\max }}\right) Y_{G}^{R T} \quad \forall g, s, t, i \\
& \mu_{g, s, R T}^{\text {down }} \geq 0, P_{g, t}^{D A}-R_{g, t, i}^{\text {down }}{ }^{s} \geq 0 \quad \forall g, s, t, i \\
& P_{g, t}^{D A}-R_{g, t, i}^{\text {down }}{ }^{s} \leq b_{g, s, R T}^{\text {down }} X_{G}^{R T} \quad \forall g, s, t, i \\
& \mu_{g, s, R T}^{\text {down }} \leq\left(1-b_{g, s, R T}^{\text {down }}\right) Y_{G}^{R T} \quad \forall g, s, t, i
\end{aligned}
$$

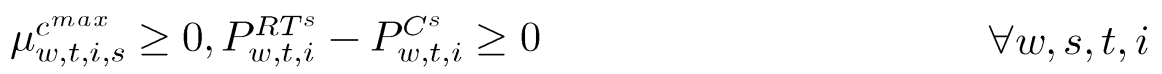

$$
\begin{aligned}
& P_{w, t, i}^{R T^{s}}-P_{w, t, i}^{C^{s}} \leq b_{w, t, i, s}^{c^{\max }} X_{W}^{R T} \quad \forall w, s, t, i \\
& \mu_{w, t, i, s}^{c^{\max }} \leq\left(1-b_{w, t, i, s}^{c^{\max }}\right) Y_{W}^{R T} \quad \forall w, s, t, i \\
& \mu_{d, t, i, s}^{c^{\max }} \geq 0, L_{d}^{\max }-L_{d, t, i}^{C^{s}} \geq 0 \quad \forall d, s, t, i \\
& L_{d}^{\max }-L_{d, t, i}^{C^{s}} \leq b_{d, t, i, s}^{c^{\max }} X_{D}^{R T} \quad \forall d, s, t, i \\
& \mu_{d, t, i, s}^{c^{\max }} \leq\left(1-b_{d, t, i, s}^{c^{\max }}\right) Y_{D}^{R T} \\
& \forall d, s, t, i \\
& \mu_{e v, t, i, s}^{u p^{\min }} \geq 0, R_{e v, t, i}^{u p^{s}} \geq 0 \\
& \forall e v, s, t, i \\
& R_{e v, t, i}^{u p^{s}} \leq b_{e v, t, i, s}^{u p^{m i n}} X_{E}^{R T} \\
& \forall e v, s, t, i \\
& \mu_{e v, t, i, s}^{u p^{m i n}} \leq\left(1-b_{e v, t, i, s}^{u p^{m i n}}\right) Y_{E}^{R T} \\
& \forall e v, s, t, i \\
& \mu_{e v, t, i, s}^{d_{\text {min }}} \geq 0, R_{e v, t, i}^{\text {down }} \geq 0 \\
& \forall e v, s, t, i \\
& R_{e v, t, i}^{d o w n^{s}} \leq b_{e v, t, i, s}^{d^{\text {min }}} X_{E}^{R T} \\
& \forall e v, s, t, i \\
& \mu_{e v, t, i, s}^{d^{m i n}} \leq\left(1-b_{e v, t, i, s}^{d^{m i n}}\right) Y_{E}^{R T} \\
& \forall e v, s, t, i \\
& \mu_{g, t, i, s}^{u p^{m i n}} \geq 0, R_{g, t, i}^{u p^{s}} \geq 0 \\
& \forall g, s, t, i \\
& R_{g, t, i}^{u p^{s}} \leq b_{g, t, i, s}^{u p^{m i n}} X_{G}^{R T}
\end{aligned}
$$




$$
\begin{array}{ll}
\mu_{g, t, i, s}^{u p^{m i n}} \leq\left(1-b_{g, t, i, s}^{u p^{m i n}}\right) Y_{G}^{R T} & \forall g, s, t, i \\
\mu_{g, t, i, s}^{d^{m i n}} \geq 0, R_{g, t, i}^{d o w n^{s}} \geq 0 & \forall g, s, t, i \\
R_{g, t, i}^{d o w n^{s}} \leq b_{g, t, i, s}^{d^{m i n}} X_{G}^{R T} & \forall g, s, t, i \\
\mu_{g, t, i, s}^{d^{m i n}} \leq\left(1-b_{g, t, i, s}^{d^{m i n}}\right) Y_{G}^{R T} & \forall g, s, t, i \\
\mu_{w, t, i, s}^{c^{m i n}} \geq 0, P_{w, t, i}^{C^{s}} \geq 0 & \forall w, s, t, i \\
P_{w, t, i}^{C^{s}} \leq b_{w, t, i, s}^{c^{m i n}} X_{W}^{R T} & \forall w, s, t, i \\
\mu_{w, t, i, s}^{c^{m i n}} \leq\left(1-b_{w, t, i, s}^{c^{m i n}}\right) Y_{W}^{R T} & \forall w, s, t, i \\
\mu_{d, t, i, s}^{c^{m i n}} \geq 0, L_{d, t, i}^{C^{s}} \geq 0 & \forall d, s, t, i \\
L_{d, t, i}^{C^{s}} \leq b_{d, t, i, s}^{c^{m i n}} X_{L}^{R T} & \forall d, s, t, i \\
\mu_{d, t, i, s}^{c^{m i n}} \leq\left(1-b_{d, t, i, s}^{c^{m i n}}\right) Y_{L}^{R T} & \forall d, s, t, i
\end{array}
$$

where $X_{E}^{R T}, X_{G}^{R T}, X_{W}^{R T}, X_{L}^{R T}, Y_{E}^{R T}, Y_{G}^{R T}, Y_{W}^{R T}, Y_{L}^{R T}$ are large enough positive constants and $b_{(.), t, i, s}^{(\cdot)}$ are binary variables.

3) Mixed-integer linear equivalents of the complementarity conditions section 4.3.2.3 from equation $(4.29 \mathrm{~b})$ to $(4.29 \mathrm{c})$ :

$$
\begin{array}{ll}
\mu_{e v, t, i, s}^{E E V^{\max }} \geq 0, E_{e v, t}^{D A}-\frac{1}{N_{I}} \sum_{s} \sum_{i} \sum_{e v} E_{e v, t}^{M a x} \cdot N_{e v, t, i}^{s} \cdot S O C_{E V}^{\max } \geq 0 & \forall e v, t \\
E_{e v, t}^{D A}-\frac{1}{N_{I}} \sum_{s} \sum_{i} \sum_{e v} E_{e v, t}^{M a x} \cdot N_{e v, t, i}^{s} . S O C_{E V}^{\max } \leq b_{e v, t, i, s}^{E E V^{\max }} X_{E E} & \forall e v, t \\
\mu_{e v, t, i, s}^{E E V^{\max }} \leq\left(1-b_{e v, t, i, s}^{E E V^{\max }}\right) Y_{E E} & \forall e v, t
\end{array}
$$




$$
\begin{array}{ll}
\mu_{e v, t, i, s}^{E E V^{m i n}} \geq 0, \frac{1}{N_{I}} \sum_{s} \sum_{i} \sum_{e v} E_{e v, t}^{M i n} \cdot N_{e v, t, i}^{s} \cdot S O C_{E V}^{m i n}-E_{e v, t}^{D A} \geq 0 & \forall e v, t \\
\frac{1}{N_{I}} \sum_{s} \sum_{i} \sum_{e v} E_{e v, t}^{M i n} \cdot N_{e v, t, i}^{s} . S O C_{E V}^{m i n}-E_{e v, t}^{D A} \leq b_{e v, t, i, s}^{E E V^{m i n}} X_{E E} & \forall e v, t \\
\mu_{e v, t, i, s}^{E E V^{m i n}} \leq\left(1-b_{e v, t, i, s}^{E E V^{m i n}}\right) Y_{E E} & \forall e v, t
\end{array}
$$

where $X_{E E}$ and $Y_{E E}$ are large enough positive constants and $b_{e v, t, i, s}^{E E V^{(.)}}$are binary variables.

\subsubsection{Linearization of Nonlinear terms}

Nonlinear terms in Profit ${ }^{s}$ can be linearized by the strong duality conditions, and KKT equalities as discussed in [21-22].

$$
\begin{aligned}
\text { Profit }^{s}=- & \sum_{t} \sum_{g} P_{g, t}^{D A} \cdot C_{g}-\sum_{t} \sum_{d} L_{d}^{\max } \cdot \mu_{d, t}^{D A^{\max }} \\
& +\sum_{d t} \sum_{d} B_{d, t}^{D A} \cdot P_{d, t}^{D A} \\
& +\frac{1}{N_{I}} \sum_{i} \sum_{t} \sum_{g} \sum_{s}\left(R_{g, t, i}^{u p^{s}}-R_{g, t, i}^{d o w n^{s}}\right) \cdot C_{g} \\
& +\frac{1}{N_{I}} \sum_{i} \sum_{t} \sum_{d} \sum_{s}\left(C_{d, t}^{L} \cdot L_{d, t, i}^{C^{s}}-L_{d}^{\max } \cdot \mu_{d, t, i, s}^{c^{\max }}\right) \\
& +\frac{1}{N_{I}} \sum_{i} \sum_{t} \sum_{e v} \sum_{s}\left(E_{e v, t, i-1}^{s}\right. \\
& \left.-\left(1-N_{e v, t, i}^{s}\right) \cdot D_{e v, t, i}^{s}\right) \cdot \rho^{T}
\end{aligned}
$$

The objective function includes only one nonlinear term $\left(E_{e v, t, i-1}^{s}\right)$ which is a bilinear one. Hence, discretising with reasonable step size is found to be a sufficiently accurate approximation as used in [24]. 


\subsection{Case Studies and Numerical Results}

To test the proposed model, the total demand is considered as $4.5 \mathrm{GWh}$ with five demand blocks of 2.25, 0.675, 0.675, 0.45, and 0.45 GWh. Fig. 4.4 provides demand bid prices for each period of time in five demand blocks. The system has a WGenCo with a single wind farm and two CGenCos including nuclear, and gas units which are assumed to be dispatchable. Generator data are listed in Table 4.1. The total power capacities of wind and dispatchable units are the percentages of a total installed CGenCo power capacity $\left(P^{\text {CGenCo }}\right)$ of $5 \mathrm{GW}$ (see Table 4.1).

The number of intra-hour intervals is 6 (i.e. $10 \mathrm{~min}$ each). Figs. 4.5 and 4.6 show the intra-hourly wind power generation forecasted and EV penetration forecasted, respectively.

Table 4.1

Summary of Data

\begin{tabular}{l|c|c}
\hline \hline Unit Type & Nuclear & Gas \\
\hline$P_{g}^{\text {Max }}(\mathrm{MW})$ & 1500 & 3500 \\
\hline Cg $(\$ / \mathrm{MWh})$ & 12 & 30 \\
\hline$R_{g, \text { Max }}^{\text {UP }} R_{g, \text { Min }}^{\text {DOWN }}\left(\%\right.$ of $\left.P_{g}^{\text {Max }}\right)$ & 0 & $50 \%$ \\
\hline Total Demand $(\mathrm{MW})$ & \multicolumn{2}{|c}{4500} \\
\hline$P^{\text {CGenCo }}(\mathrm{MW})$ & \multicolumn{2}{|c}{5000} \\
\hline Total Wind $(\mathrm{MW})$ & $20 \%$ of $P^{\text {CGenCo }}$ \\
\hline
\end{tabular}




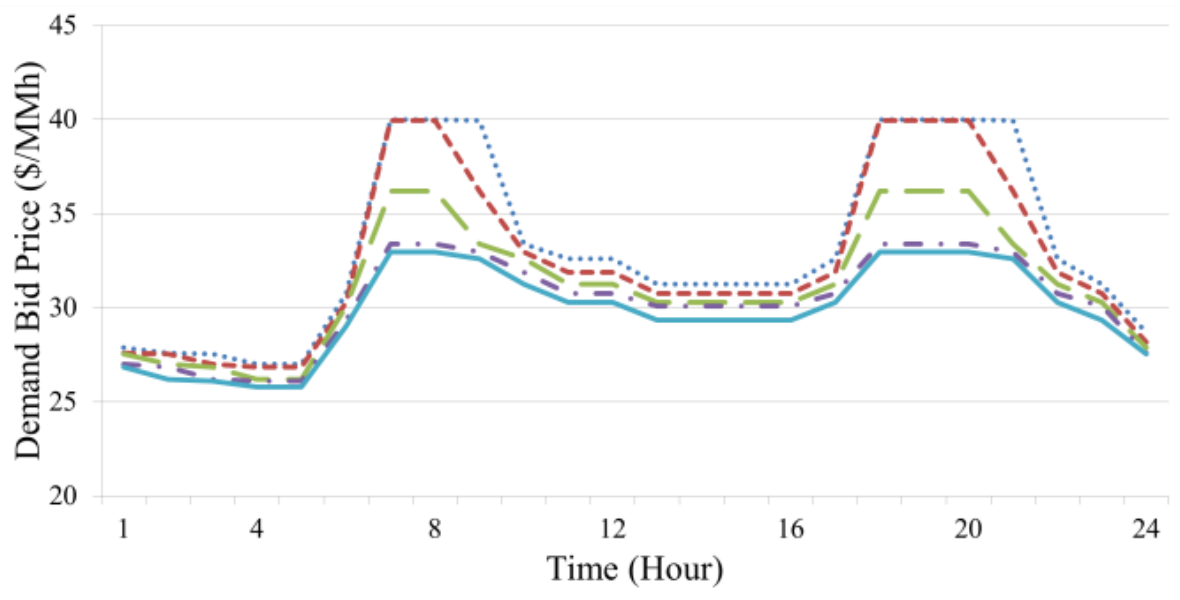

Fig. 4.4 Demand bid prices in five demand blocks

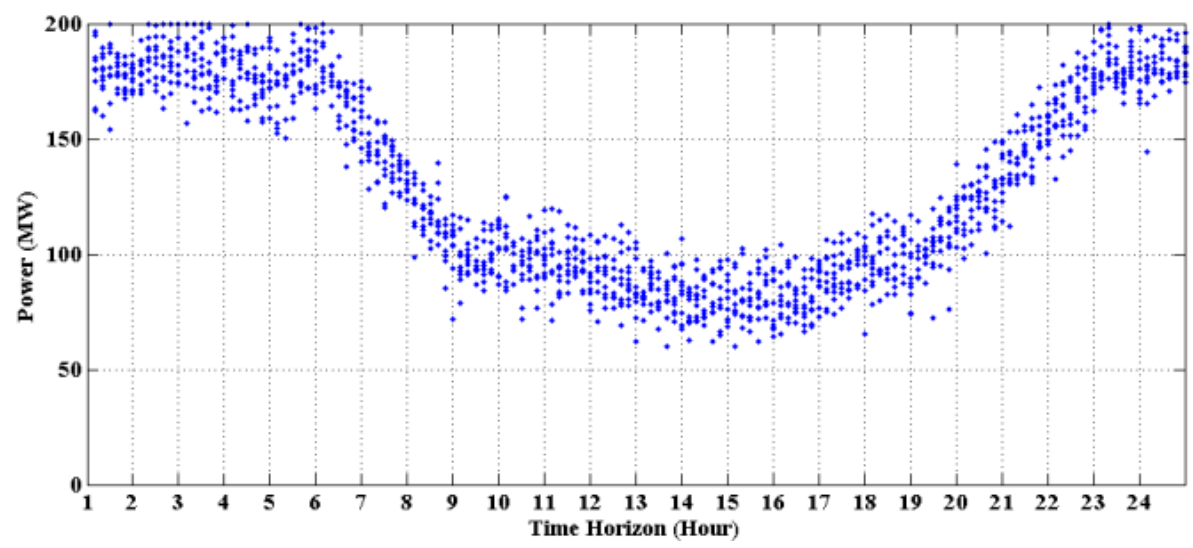

Fig. 4.5 The intra-hourly wind power generation forecasted for ten scenarios.

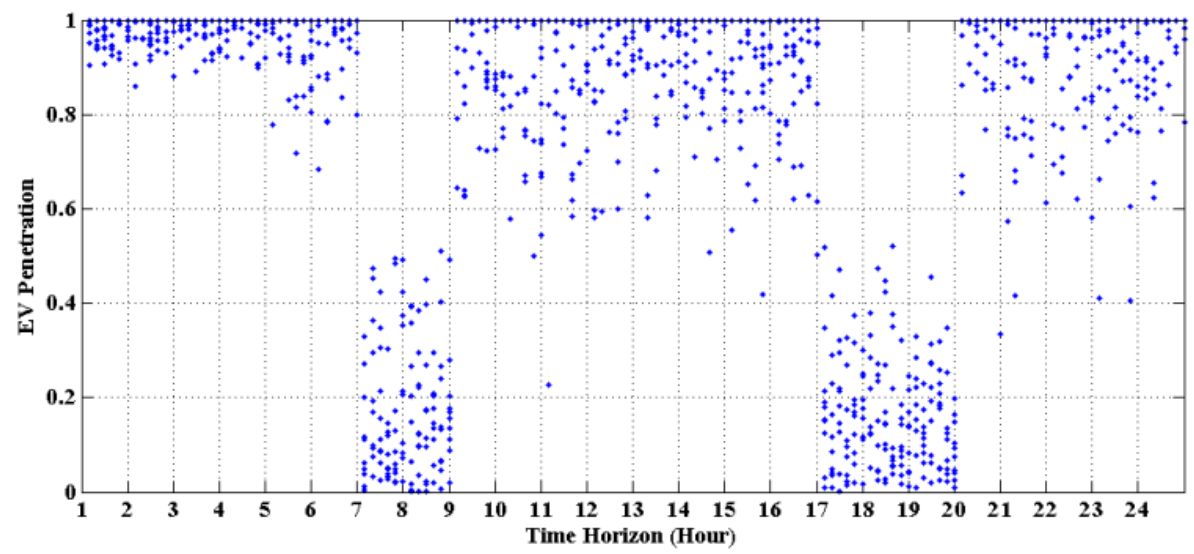

Fig. 4.6 The intra-hourly EV penetration forecasted for ten scenarios. 
The maximum EV charging power is assumed to be $7.3 \mathrm{~kW}$, and the energy capacity of each EV is $27.4 \mathrm{kWh}$. Average annual driving distance of an EV is assumed to be $20,000 \mathrm{~km}$ with an average daily distance of $52.91 \mathrm{~km}$. The required energy for an $\mathrm{EV}$ is $9 \mathrm{kWh} /$ day with an average of $5.87 \mathrm{~km} / \mathrm{kWh}$ [30]. We assume that the required energy for driving in one direction is the same as that of returning to the starting point. For the EV aggregator, we consider different EV numbers from one thousand to one hundred thousand. The EV fleet has its own commute time based on factors including region, city, and traffic

patterns. In this chapter, the number of EV fleets is assumed to be one with commute intervals between 7 A.M. and 9 A.M., and between P.M. and 8 P.M.

The proposed model is utilized to consider endogenous tariffs. In this context, both fixed-rate and ToU tariffs and the impact of the tariffs on the wholesalelevel market output are studied. In the fixed-rate tariff, the rate of charges and payments of an EV owner are considered to be fixed. Contrariwise, in ToU tariff this rate changes in different periods of the day including off-peak, peak, and base [11].

The market output in terms of the payoff, price, and generation and demand dispatch is analyzed.

\subsubsection{Payoff and Price Analysis}

The WGenCo profit and total payoff versus EV numbers at both fixed-rate and ToU tariffs are shown in Figs. 4.7 and 4.8 respectively. The day-ahead equilibrium prices with respect to 50,000 and 100,000 EV numbers, and using fixed-rate and ToU tariffs are shown in Figs. 4.9-4.12, respectively.

Comparing Figs. 4.9 and 4.11 shows that the increase in the EV number results in the decrease in the day-ahead equilibrium price because of the imposed 
fixed-rate tariff. On the basis of this, the firm's total expected profit decreases with more EV numbers using fixed-rate tariff as shown in Fig. 4.8. However, using the ToU tariff increases the firm's expected profit with higher penetration of EVs. Comparing Figs. 4.10 and 4.12 shows that the increase in the EV number does not change the day-ahead equilibrium price because of the greater flexibility of the ToU tariff. Fig. 4.7 shows that the WGenCo's profit increases with higher penetration of EVs for two kinds of tariffs. It means that the increased EV number results in more wind energy contribution in the market.

The EV aggregator's profit is obtained through purchasing energy from the market and selling it to EV owners at a certain tariff. According to Figs. 4.10 and 4.12, using the ToU tariff reduces the difference between DA price and ToU tariffs (see Figs. 4.9-4.12), and then decreases the EV aggregator's profit in comparison with using the fixed-rate tariff (see Table 4.2). 


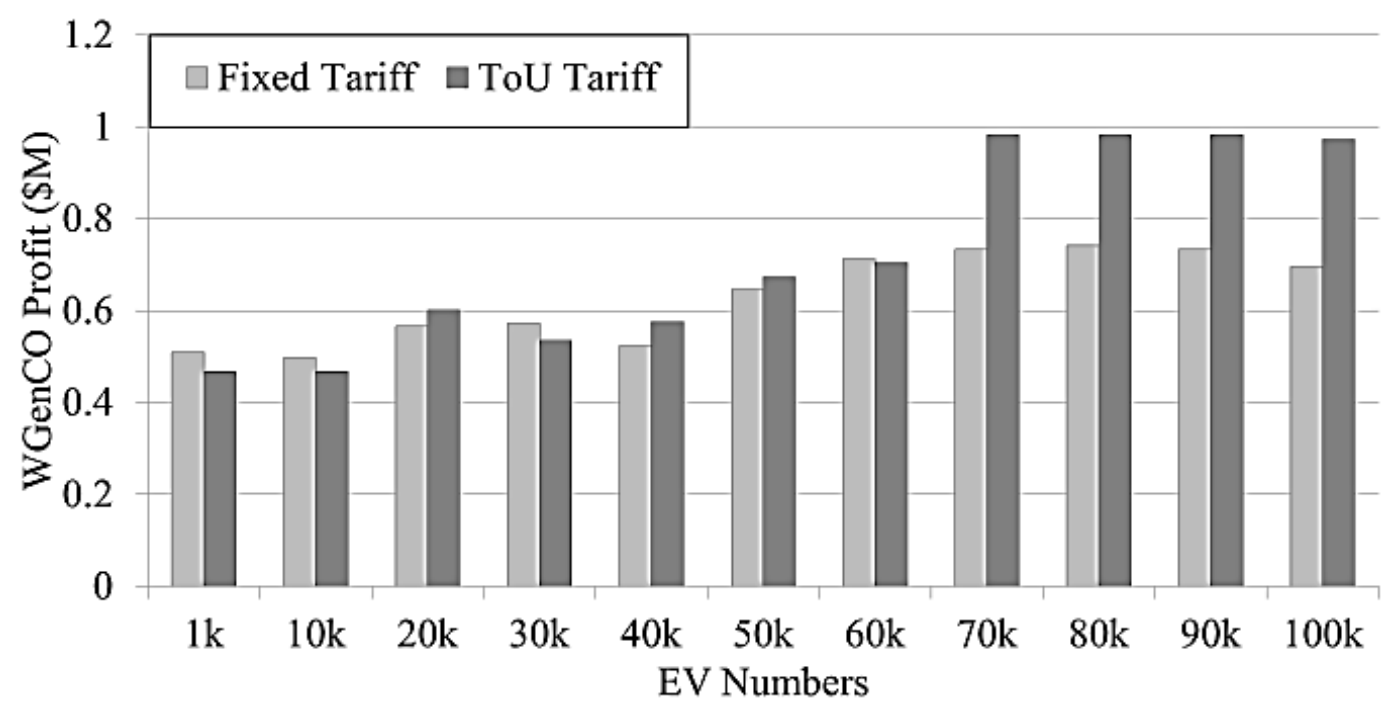

Fig. 4.7. Impact of EV numbers and tariffs on WGenCo profit

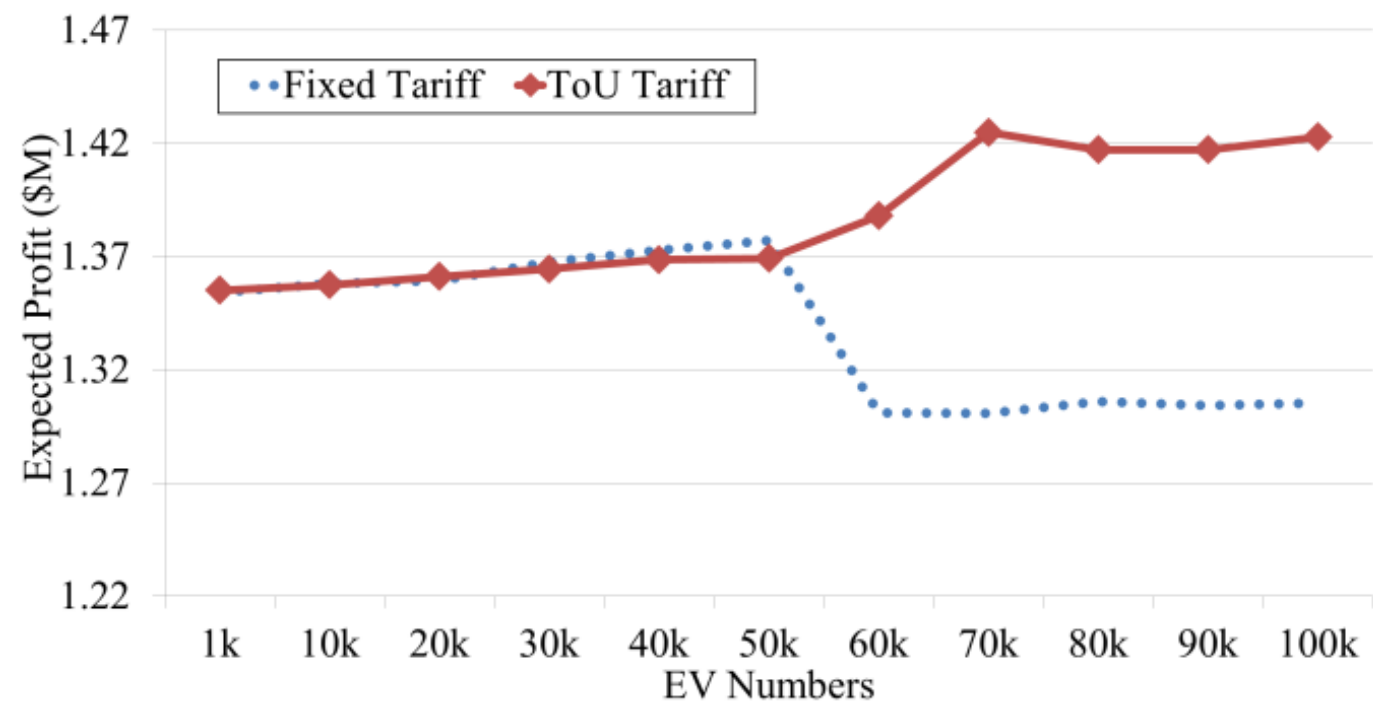

Fig. 4.8. Impact of EV numbers and tariffs on the total profit

Table 4.2

EV Aggregator's Profit

\begin{tabular}{c|c|c|c|c}
\hline & $1 \mathrm{k} \mathrm{EV}$ & $30 \mathrm{k} \mathrm{EV}$ & $50 \mathrm{k} \mathrm{EV}$ & $100 \mathrm{k} \mathrm{EV}$ \\
\hline \hline Fixed-rate tariff & 1,247 & 15,498 & 27,871 & 52,197 \\
\hline ToU tariff & 463 & $-1,986$ & -742 & $-2,995$ \\
\hline
\end{tabular}




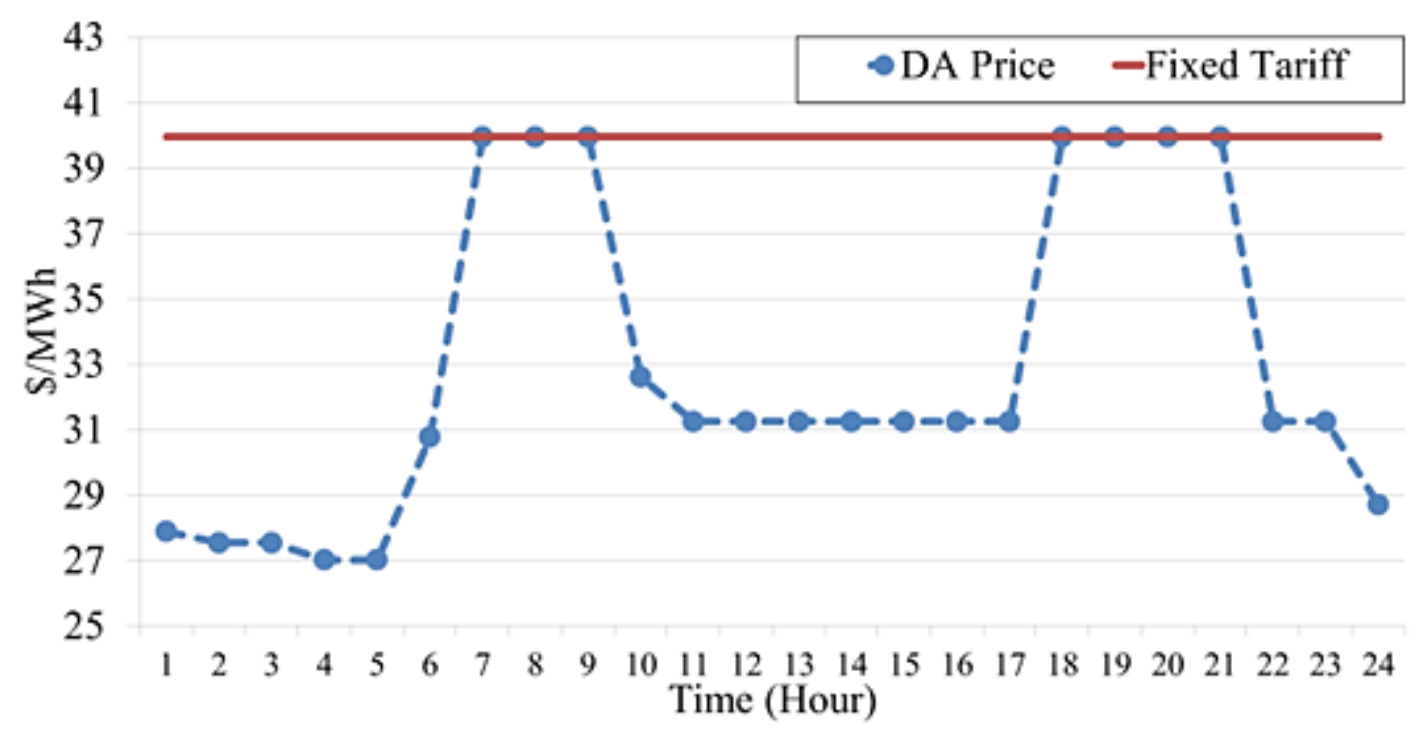

Fig. 4.9. Impact of fixed-rate tariff and 50,000 EVs on the day-ahead price

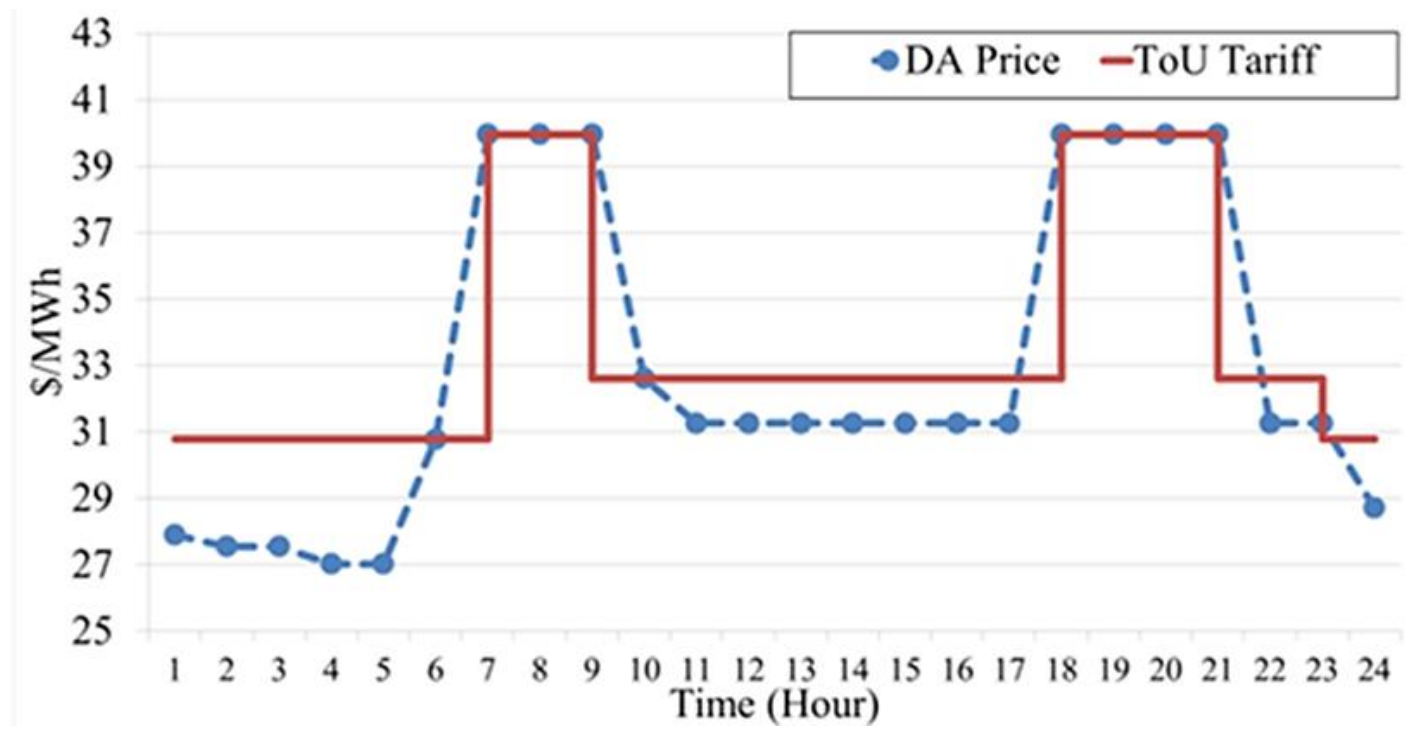

Fig. 4.10. Impact of ToU tariff and 50,000 EVs tariff on the day-ahead price 


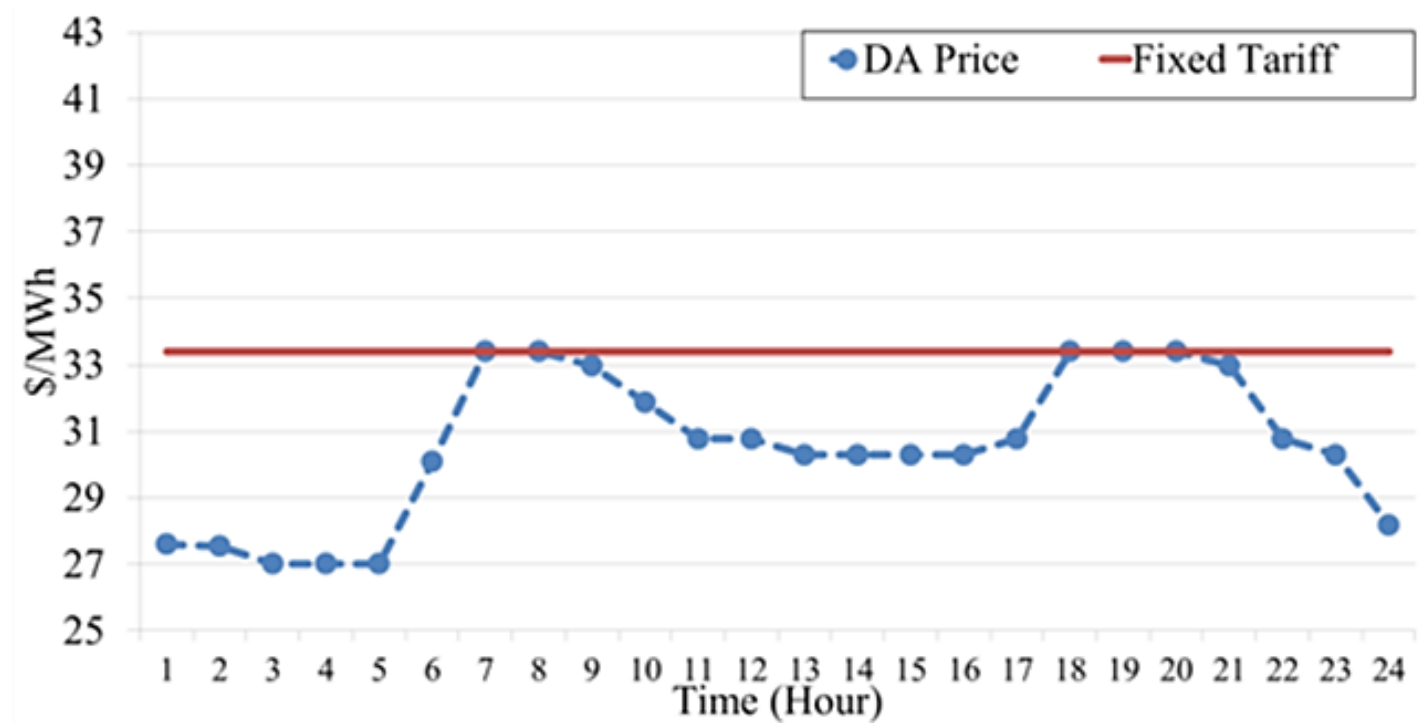

Fig. 4.11. Impact of fixed-rate tariff and 100,000 EVs on the day-ahead price

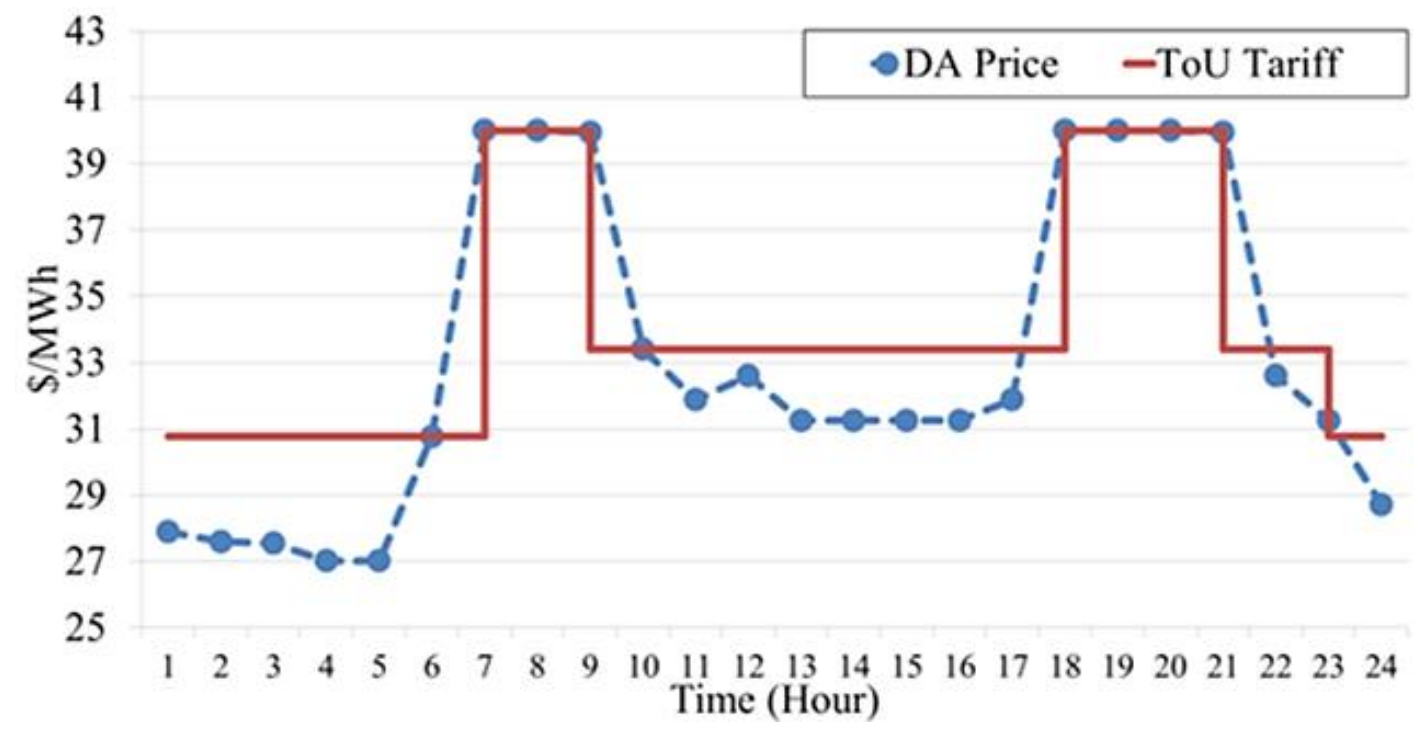

Fig. 4.12. Impact of ToU tariff and 100,000 EVs on the day-ahead price 


\subsubsection{Demand and Generation Dispatch Analysis}

The conventional and wind power generations, and EV and load demand schedule with respect to 50,000 and 100,000 EV numbers, and fixed-rate and ToU tariffs are shown in Figs. 4.13 -4.16, respectively. As is clear from these figures, the required EV demand is supplied from the WGenCo. Therefore, it avoids shifting the emissions of the transportation sector to the electricity sector. It can be observed that the EV aggregator prefers to charge EVs during off peak between 1 A.M. to 6 A.M. Indeed, the difference between the EV tariff and the market price in these hours is high and EVs are available to be charged.

By comparing fixed-rate and ToU tariff in Fig. 4.17, it is obvious that the increase in the EV number enhances the total demand and CGenCo power because of the imposed fixed-rate tariff and less DA market price. However, using ToU tariff does not affect the total demand and CGenCo power with higher penetration of EV due to the flexibility of the ToU tariff and the consistency in the DA market price.

On the other hand, wind power penetration increases through greater numbers of EVs for two kinds of tariff as shown in Fig. 4.18. In addition, Fig. 4.19 shows the reduction in the wind curtailment with higher penetration of EVs. 


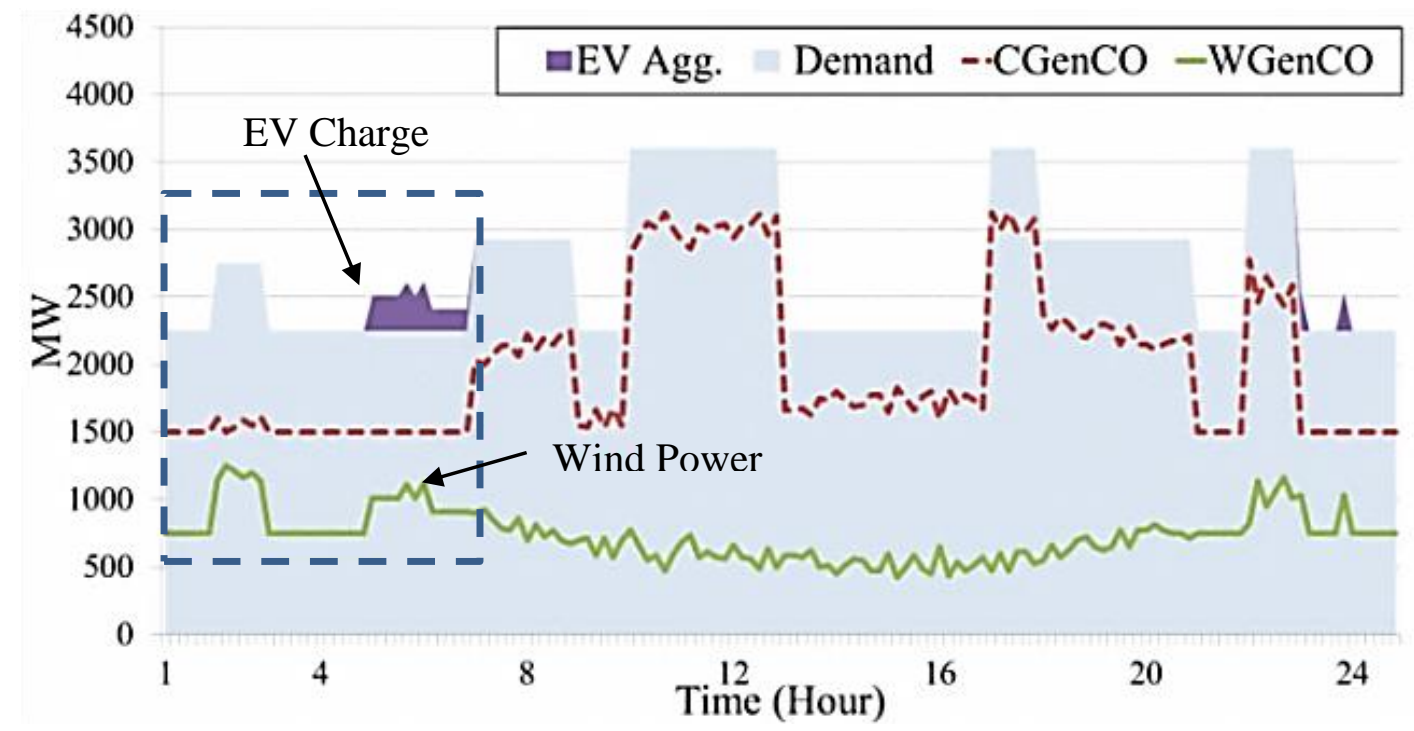

Fig. 4.13. Impact of fixed-rate tariff and 50,000 EVs on generation and demand dispatch

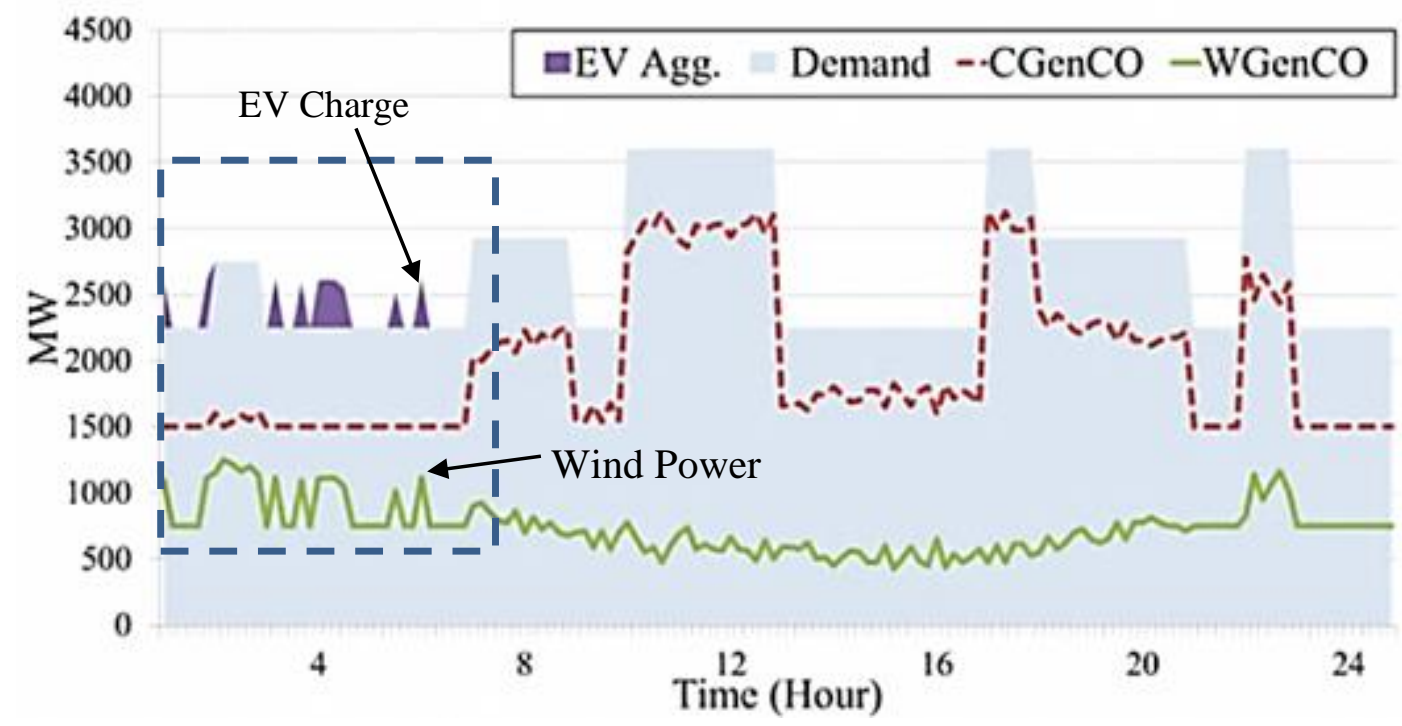

Fig. 4.14. Impact of ToU tariff and 50,000 EVs on generation and demand dispatch 


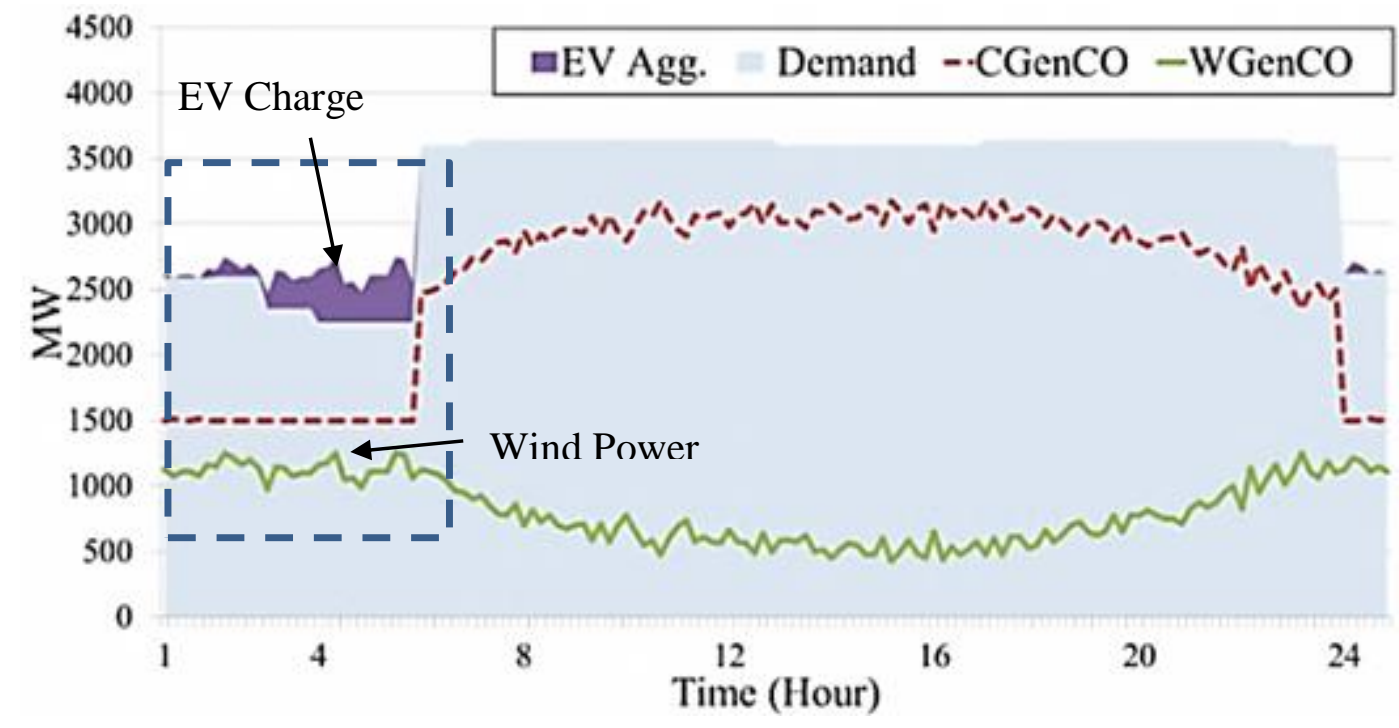

Fig. 4.15. Impact of fixed-rate tariff and 100,000 EVs on generation and demand dispatch

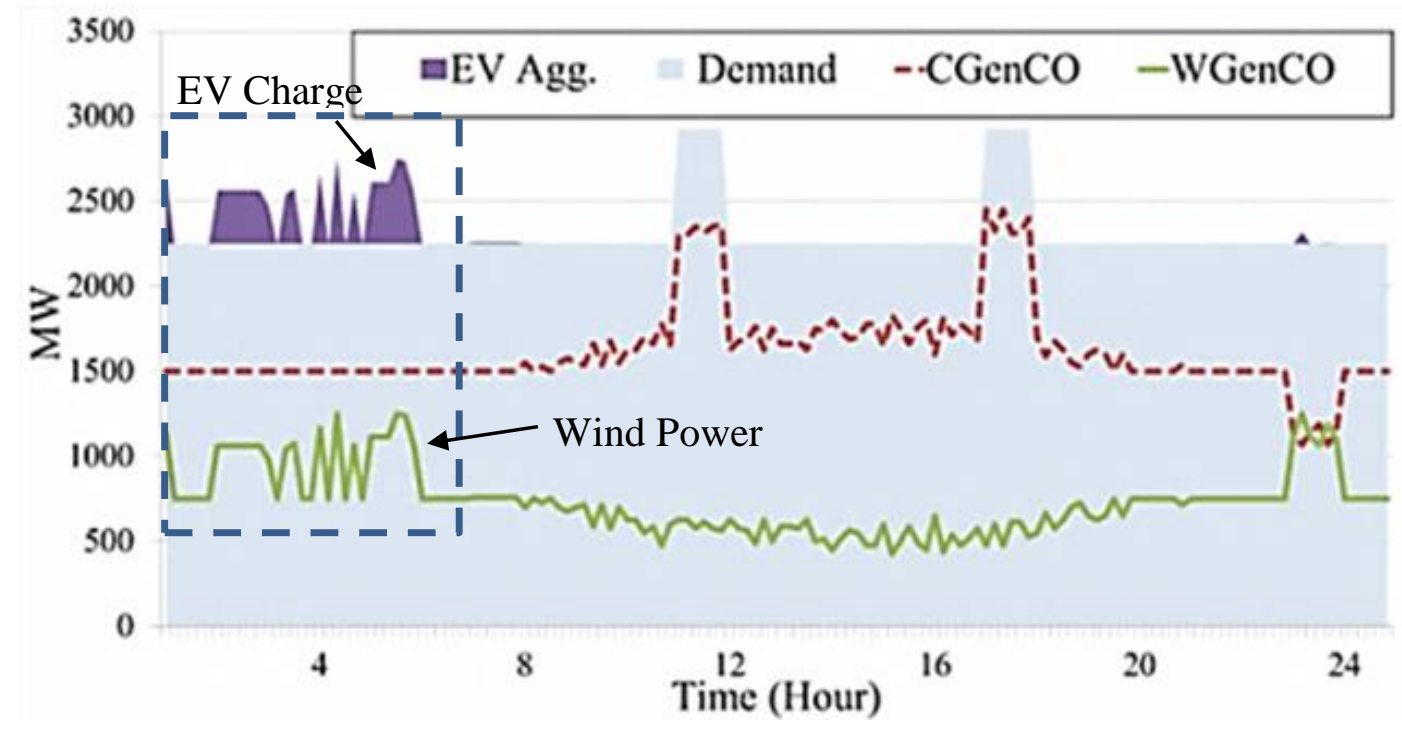

Fig. 4.16. Impact of ToU tariff and 100,000 EVs on generation and demand dispatch 


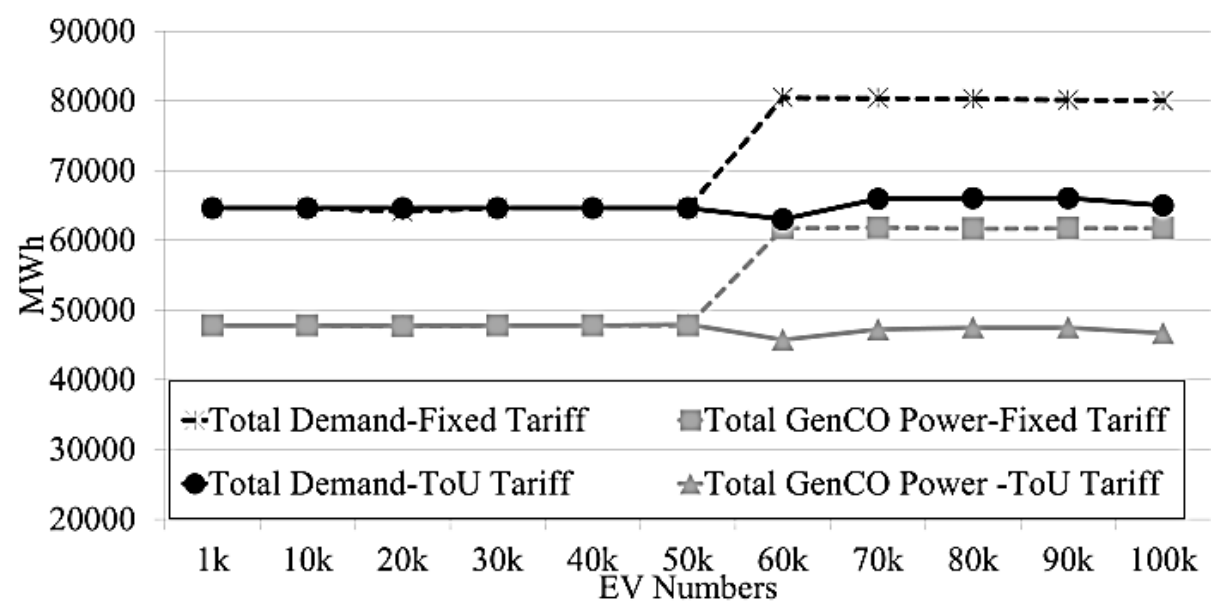

Fig. 4.17. Impact of tariffs and EV numbers on total demand and CGenCo power generation

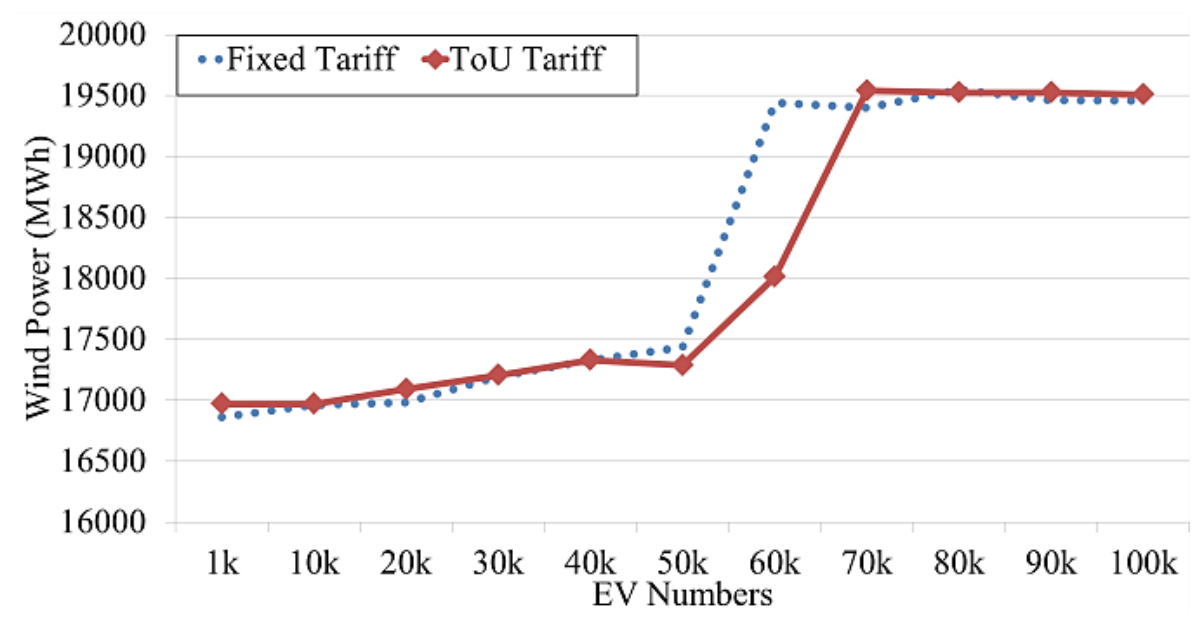

Fig. 4.18. Impact of tariffs and EV numbers on wind power generation

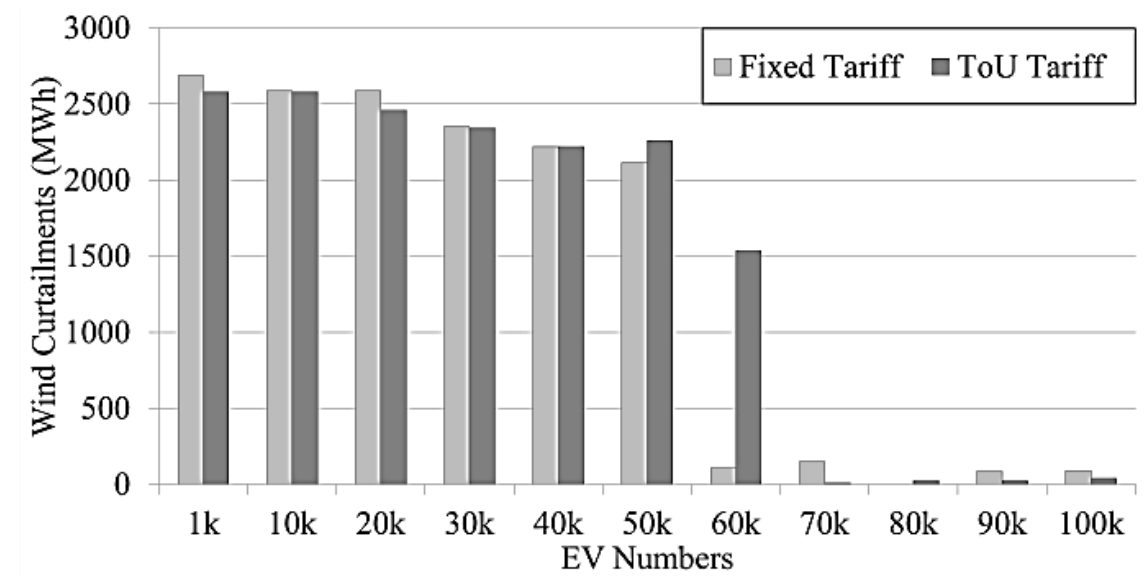

Fig. 4.19. Impact of tariffs and EV numbers on wind curtailment 


\subsection{Summary}

In this chapter, two levels are introduced as wholesale-level and EV-level in the electricity market. At the wholesale-level, an optimal bidding/offering strategy for the EV load aggregator providing the energy and ancillary services in coordination with a strategic producer in a pool-based electricity market is modelled. At the EV-level, EV owners connect to the EV aggregator in order to take part in the market indirectly for obtaining maximum EV energy capacity and optimal charging tariff based on achieved day-ahead and real-time market data.

The proposed formulation of a stochastic intra-hour bilevel optimization problem is given by an MPEC. The MPEC includes the strategic firm's profit maximization as an upper-level problem, subject to three lower level problems: DA and RT social welfare maximizations, and the EV owners' battery energy maximization. The impact of EV numbers, and using both fixed-rate and ToU tariff on the price and market outcome are investigated. The results show that the EV-level market outputs affect the behaviour of the wholesale-level market.

Through the model, the required EV charging demand is supplied by WGenCo (wind energy) in order to reduce the emissions in transportation and electricity segments. Furthermore, high penetration of EVs leads to increasing wind power generation and reducing wind power curtailment.

The EV tariff and numbers and their trading at the EV-level can influence the market price and power generation at the wholesale-level. As the EV aggregator has to purchase energy from the wholesale-level market with uncertainty in prices and sell it to EV customers, using ToU tariffs can reduce this influence. 


\section{Chapter 5}

\section{Pool Strategy of Multiple Firms in}

\section{Coordination with EV Load Aggregators}

\subsection{Introduction}

The objective of this chapter is to develop the coordination strategy for the EV load aggregator with the CGenCos and WGenCos in multiple strategic firms competing with each other.

Similarly to Chapter 4, CGenCos, WGenCos, and EV aggregators considered in this chapter are strategic. An optimal bidding/offering strategy is developed for the EV load aggregator providing the energy and ancillary services. The optimal offering strategies are developed for CGenCos and WGenCos.

The rest of this chapter is organized as follows. Section 5.2 discusses the market framework and approach. Section 5.3 provides a formulation of a bilevel 
model. Test of the proposed market model through case studies is described in Section 5.4. Finally, Section 5.5 concludes, summarizing the chapter.

\subsection{Market Framework and Approach}

The market frame used in this chapter is similar to one proposed in Chapter 4 , in spite of multiple strategic firms. At the wholesale-level, strategic firms including CGenCos, WGenCos, and EV aggregators submit supply-offers/demand-bids to the MO to participate in the day-ahead and real-time market directly. At the EV-level, EV owners connect to each EV aggregator in order to take part in the market indirectly. EV aggregators maximize the EV energy capacities and determine optimal charging tariff based on achieved day-ahead and real-time data. Fig. 5.1 shows two levels of market framework are introduced in this chapter which are wholesale-level and EV-level for multiple strategic firms.

However, an EPEC is established to consider all strategic firms in this chapter. Similarly to Chapter 4, the upper-level problem of the bilevel model maximizes the expected profit of each strategic firm, and its lower-level problems represent different market clearing. Then, substituting the lower-level problems with their optimality conditions in the single firm model extracts an MPEC for each strategic firm. The combination of multiple firms MPEC constitutes an EPEC. The optimality conditions of the EPEC are linearized by formulating and solving a MILP problem. Finally, a diagonalization algorithm is executed to verify each solution as a real Nash equilibrium. Fig. 5.2 shows six steps to determine the market equilibria. 


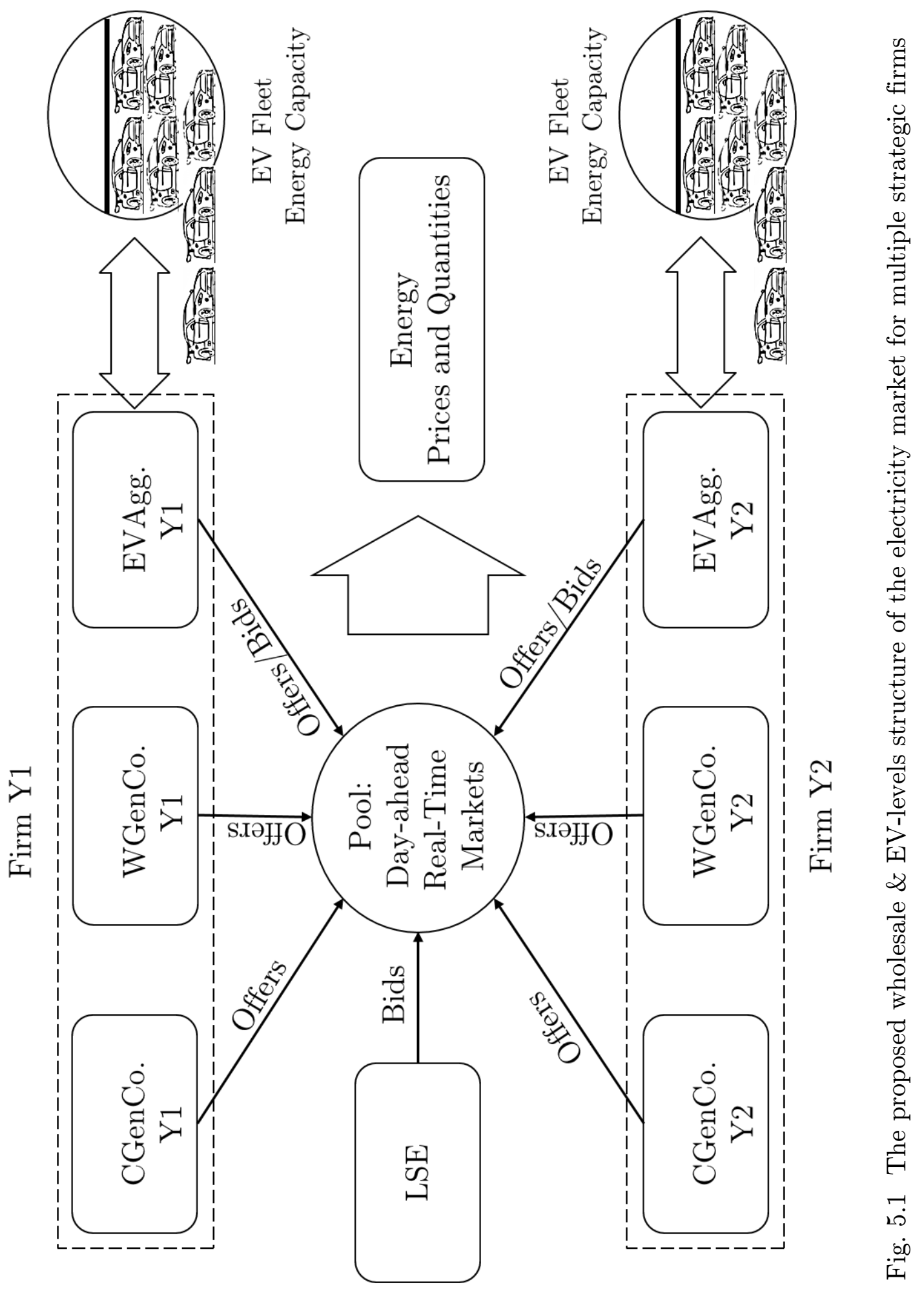




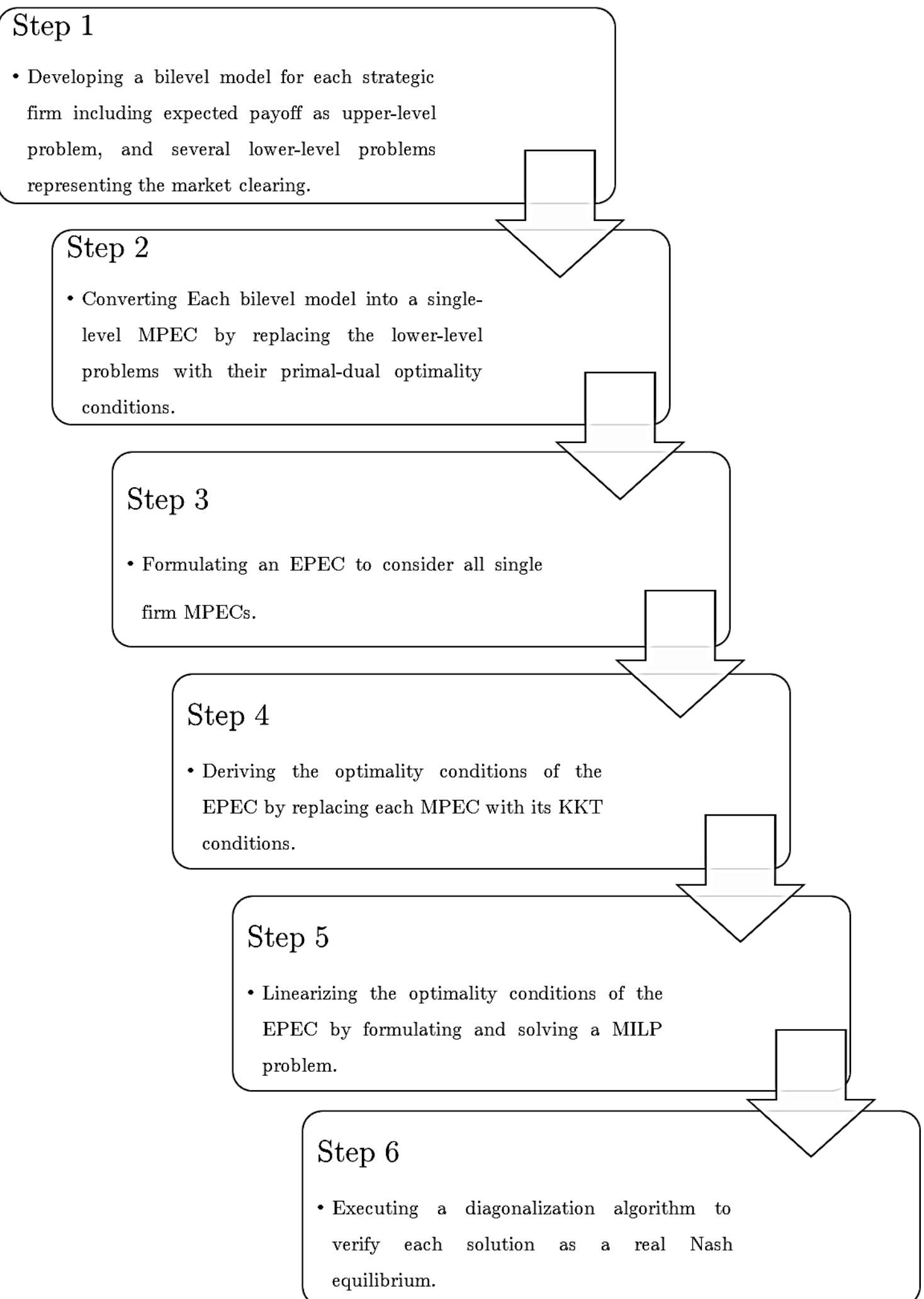

Fig. 5.2 The steps to obtain the market equilibria 


\subsection{Formulation of Bilevel Model}

The single-period bilevel problem for each strategic firm $\mathrm{Y}$ is constructed and then converted into a single MPEC. Finally, the EPEC problem is formulated.

The single-period objective function (5.1a) maximizes the expected profits of each strategic firm Y which owns both CGenCos and WGenCos and constitutes unidirectional V2G services. Similar to Chapter 4 Subsections 4.3.1.1-4, three single-period lower-level problems including the day-ahead market, real-time market, and EV energy market clearing, which aim to maximize the social welfare and are subject to the power balance, and power constraints, are presented as follows:

$$
\begin{aligned}
& \underset{\Omega_{\text {prim }}, \Omega_{\text {dual }}}{\operatorname{Max}} \text { Profit }^{s} \\
&=\sum_{g \in y} P_{g}^{D A} \cdot\left(\rho^{D A}-C_{g}\right)+\sum_{w \in y} P_{w}^{D A} \cdot \rho^{D A} \\
&-\sum_{e v \in y} P O P_{e v} \rho^{D A}-\rho^{T} \\
&+\pi_{s} \sum_{s}\left(\sum_{g \in y}\left(R_{g}^{u p^{s}}-R_{g}^{d o w n^{s}}\right)\left(\rho_{s}^{R T}-C_{g}\right)\right. \\
&-\sum_{w \in y} P_{w}^{D A}+P_{w}^{C^{s}}-P_{w}^{R T^{s}} \cdot \rho_{s}^{R T} \\
&\left.+\sum_{e v \in y} R_{e v}^{u p^{s}}-R_{e v}^{d o w n^{s}} \rho_{s}^{R T}-\rho^{T}\right)
\end{aligned}
$$

Subject to:

$$
\begin{array}{ll}
\alpha_{g}^{D A} \geq 0 & \forall g \in y \\
\alpha_{w}^{D A} \geq 0 & \forall w \in y \\
\beta_{e v}^{D A} \geq 0 & \forall e v \in y \\
\alpha_{g}^{R T^{s}} \geq 0 & \forall g \in y, \forall s \\
\alpha_{w}^{R T^{s}} \geq 0 & \forall w \in y, \forall s
\end{array}
$$




$$
\begin{aligned}
& \alpha_{e v}^{R T^{s}} \geq 0 \\
& \left\{\operatorname{Min}_{\left\{\Omega_{\text {prim }}^{D A}, \Omega_{\text {dual }}^{D A}\right\}}\left[\begin{array}{c}
\sum_{g} \alpha_{g}^{D A} P_{g}^{D A}+\sum_{w} \alpha_{w}^{D A} P_{w}^{D A}- \\
\sum_{d} B_{d}^{D A} L_{d}^{D A}-\sum_{e v} \beta_{e v}^{D A} P O P_{e v}
\end{array}\right]\right. \\
& \sum_{g} P_{g}^{D A}+\sum_{w} P_{w}^{D A}-\sum_{d} L_{d}^{D A}-\sum_{e v} P O P_{e v}=0 \quad: \rho^{D A} \\
& 0 \leq P O P_{e v} \leq P_{e v}^{\max } \\
& : \mu_{e v}^{D A^{\max }}, \mu_{e v}^{D A^{\text {min }}} \quad \forall e v \\
& 0 \leq P_{g}^{D A} \leq P_{g}^{\max } \\
& : \mu_{g}^{D A^{\max }}, \mu_{g}^{D A^{\text {min }}} \\
& \forall g \\
& 0 \leq P_{w}^{D A} \leq P_{w}^{\max }
\end{aligned}
$$

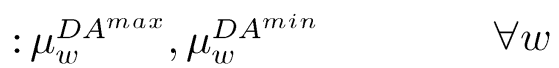

$$
\begin{aligned}
& 0 \leq L_{d}^{D A} \leq L_{d}^{\max } \\
& \left.: \mu_{d}^{D A^{\max }}, \mu_{d}^{D A^{\min }} \quad \forall d\right\} \\
& \left\{\operatorname{Min}_{\left\{\Omega_{p r i m}^{R T}, \Omega_{d u a l}^{R T}\right\}}\left[\begin{array}{c}
\sum_{g}\left(R_{g}^{u p^{s}}-R_{g, t, i}^{\text {down }}\right) \cdot \alpha_{g}^{R T^{s}}+ \\
\sum_{e v} R_{e v}^{u p^{s}}-R_{e v}^{d o w n^{s}} \cdot \alpha_{g}^{R T^{s}}+ \\
\sum_{w}\left(P_{w}^{C^{s}} \cdot \alpha_{w}^{R T^{s}}\right)+\sum_{d} C_{d}^{L} L_{d}^{C^{s}}
\end{array}\right]\right. \\
& \sum_{w} P_{w}^{D A}+P_{w}^{C^{s}}-P_{w}^{R T^{s}}-\sum_{g}\left(R_{g}^{u p^{s}}-R_{g}^{\text {down }}\right)- \\
& \sum_{d} L_{d}^{C^{s}}-\sum_{e v} R_{e v}^{u p^{s}}-R_{e v}^{d o w n^{s}}=0 \\
& : \rho_{s}^{R T} \\
& 0 \leq R_{e v}^{u p^{s}} \leq R_{e v}^{u p^{\max }} \quad: \mu_{e v, s}^{u p^{\max }}, \mu_{e v, s}^{u p^{\min }} \quad \forall e v, s \\
& R_{e v}^{u p^{s}} \leq P O P_{e v} \quad: \mu_{e v, s}^{u p} \quad \forall e v, s \\
& 0 \leq R_{e v}^{d o w n^{s}} \leq R_{e v}^{d^{\text {max }}} \quad: \mu_{e v, s}^{d^{\text {max }}}, \mu_{e v, s}^{d^{\text {min }}} \quad \forall e v, s \\
& P O P_{e v}+R_{e v}^{\text {down }} \leq P_{e v}^{\max } \quad: \mu_{e v, s}^{\text {down }} \quad \forall e v, s \\
& 0 \leq R_{g}^{u p^{s}} \leq R_{g}^{u p^{\max }} \quad: \mu_{g, s}^{u p^{\max }}, \mu_{g, s}^{u p^{\text {min }}} \quad \forall g, s \\
& P_{g}^{D A}+R_{g}^{u p^{s}} \leq R_{g}^{u p^{\max }} \quad: \mu_{e v, s}^{u p} \quad \forall g, s \\
& 0 \leq R_{g}^{\text {down }} \leq R_{g}^{d^{\text {max }}} \quad: \mu_{g, s}^{d^{\text {max }}}, \mu_{g, s}^{d^{\text {min }}} \quad \forall g, s \\
& R_{g}^{\text {down }} x^{s} P_{g}^{D A} \quad: \mu_{g s,}^{\text {down }} \quad \forall g, s \\
& 0 \leq P_{w}^{C^{s}} \leq P_{w}^{R T^{s}} \quad: \mu_{w, s}^{c^{\max }}, \mu_{w, s}^{c^{\min }} \quad \forall w, s \\
& \left.0 \leq L_{d}^{C^{s}} \leq L_{d}^{\max } \quad: \mu_{d, s}^{c^{\max }, \mu_{d, s}^{c^{\min }}} \quad \forall d, s \quad\right\} \\
& \left\{\underset{\left\{E_{e v}^{D A}, \Omega_{d u a l}^{E V}\right\}}{\operatorname{Min}}\left[-\sum_{e v} \rho^{D A} E_{e v}^{D A}\right]\right. \\
& \sum_{e v} E_{e v}^{D A}-\sum_{e v} E_{0}-1-N_{e v}^{s} \cdot D_{e v}^{s}
\end{aligned}
$$




$$
\begin{array}{ll}
-\sum_{e v} P O P_{e v}-R_{e v}^{u p^{s}}+R_{e v}^{d o w n^{s}}=0 & : \rho^{T} \\
E_{e v}^{D A}=\pi_{s} \sum_{s} \sum_{i} E_{e v}^{s} & \forall e v \\
E_{e v}^{M i n} \leq E_{e v}^{D A} \leq E_{e v}^{M a x} & \forall e v, s \quad\}
\end{array}
$$

The dual variables are shown at the corresponding constraints following a colon.

\subsubsection{Mathematical Program with Equilibrium Constraints}

The stochastic bilevel model including multi-optimization problems transforms into a single optimization problem as a single-level stochastic MPEC as follows:

$$
\begin{aligned}
& \min _{\Omega_{\text {prim }}, \Omega_{d u a l}}-\sum_{g \in y} P_{g}^{D A} \cdot\left(\rho^{D A}-C_{g}\right)-\sum_{w \in y} P_{w}^{D A} \cdot \rho^{D A}+ \\
& \sum_{e v \in y} P O P_{e v, t} \rho^{D A}-\rho^{T}-\pi_{s} \sum_{s}\left(\sum _ { g \in y } ( R _ { g } ^ { u p ^ { s } } - R _ { g } ^ { \text { down } } ) \left(\rho_{s}^{R T}-\right.\right. \\
& \left.C_{g}\right)+\sum_{w \in y} P_{w}^{D A}+P_{w}^{C^{s}}-P_{w}^{R T^{s}} \cdot \rho_{s}^{R T}-\sum_{e v \in y} R_{e v}^{u p^{s}}- \\
& \left.R_{e v}^{\text {down }} \rho_{s}^{R T}-\rho^{T}\right)
\end{aligned}
$$

The lower problems are continuous linear and they can be replaced by their KKT conditions.

$$
\begin{array}{lll}
\alpha_{g}^{D A} \geq 0 & : Z \alpha_{g, j}^{D A} & \forall g \in y \\
\alpha_{w}^{D A} \geq 0 & : Z \alpha_{w, j}^{D A} & \forall w \in y \\
\beta_{e v}^{D A} \geq 0 & : Z \beta_{e v, j}^{D A} & \forall e v \in y \\
\alpha_{g}^{R T^{s}} \geq 0 & : Z \alpha_{g, j}^{R T^{s}} & \forall g \in y, \forall s \\
\alpha_{w}^{R T^{s}} \geq 0 & : Z \alpha_{w, j}^{R T^{s}} & \forall w \in y, \forall s \\
\alpha_{e v}^{R T^{s}} \geq 0 & : Z \alpha_{e v, j}^{R T^{s}} & \forall e v \in y, \forall s \\
\sum_{g} P_{g}^{D A}+\sum_{w} P_{w}^{D A}-\sum_{d} L_{d}^{D A}-\sum_{e v} P O P_{e v}=0 & : \delta_{j}^{D A} \\
0 \leq P O P_{e v} \leq P_{e v}^{\max } & : Z_{e v, j}^{D A^{\text {max }}}, Z_{e v, j}^{D A^{\text {min }}} \forall e v \\
0 \leq P_{g}^{D A} \leq P_{g}^{\max } & : Z_{g, j}^{D A^{\text {max }}}, Z_{g, j}^{D A^{\text {min }}} \forall g
\end{array}
$$




$$
\begin{aligned}
& 0 \leq P_{w}^{D A} \leq P_{w}^{\max } \quad: Z_{w, j}^{D A^{\max }}, Z_{w, j}^{D A^{\min }} \forall w \\
& 0 \leq L_{d}^{D A} \leq L_{d}^{\max } \quad: Z_{d, j}^{D A^{\max }}, Z_{d, j}^{D A^{\min }} \forall d \\
& -\beta_{e v}^{D A}+\rho^{D A}+\mu_{e v}^{D A^{\max }}-\mu_{e v}^{D A^{m i n}}=0 \quad: Z P O P_{e v, j} \quad \forall e v \\
& +\alpha_{g}^{D A}-\rho^{D A}+\mu_{g}^{D A^{\max }}-\mu_{g}^{D A^{\text {min }}}=0 \quad: Z P_{g, j}^{D A} \quad \forall g \\
& +\alpha_{w}^{D A}-\rho^{D A}+\mu_{w}^{D A^{\text {max }}}-\mu_{w}^{D A^{\text {min }}}=0 \quad: Z P_{w, j}^{D A} \quad \forall w \\
& -B_{d}^{D A}+\rho^{D A}+\mu_{d}^{D A^{m a x}}-\mu_{d}^{D A^{m i n}}=0 \quad: Z L_{d, j}^{D A} \quad \forall d \\
& \mu_{e v}^{D A^{\min }} \geq 0, \mu_{e v}^{D A^{\max }} \geq 0 \quad: X_{e v, j}^{D A^{\text {min }}}, X_{e v, j}^{D A^{\text {max }}} \forall e v \\
& \mu_{g}^{D A^{\min }} \geq 0, \mu_{g}^{D A^{\max }} \geq 0 \quad: X_{g, j}^{D A^{\min }}, X_{g, j}^{D A^{\max }} \forall g \\
& \mu_{w}^{D A^{\min }} \geq 0, \mu_{w}^{D A^{\max }} \geq 0 \quad: X_{w, j}^{D A^{\min }}, X_{w, j}^{D A^{\max }} \forall w \\
& \mu_{d}^{D A^{m i n}} \geq 0, \mu_{d}^{D A^{\max }} \geq 0 \quad: X_{d, j}^{D A^{\text {min }}}, X_{d, j}^{D A^{\text {max }}} \forall d \\
& \sum_{g} \alpha_{g}^{D A} P_{g}^{D A}+\sum_{w, t} \alpha_{w}^{D A} P_{w}^{D A}-\sum_{d} B_{d}^{D A} L_{d}^{D A}-\sum_{e v} \beta_{e v}^{D A} P O P_{e v} \\
& \sum_{e v} \mu_{e v}^{D A^{\max }} P_{e v}^{\max }+\sum_{g} \mu_{g}^{D A^{\max }}\left(P_{g}^{\max }\right)+ \\
& \sum_{w} \mu_{w}^{D A^{\max }}\left(P_{w}^{\max }\right)+\sum_{d} \mu_{d}^{D A^{\max }}\left(L_{d}^{\max }\right)=0 \quad: \emptyset_{j}^{D A} \\
& \sum_{w} P_{w}^{D A}+P_{w}^{C^{s}}-P_{w}^{R T^{s}}-\sum_{g}\left(R_{g}^{u p^{s}}-R_{g}^{d o w n^{s}}\right)- \\
& \sum_{d} L_{d}^{C^{s}}-\sum_{e v} R_{e v}^{u p^{s}}-R_{e v}^{d o w n^{s}}=0 \quad: \delta_{j, s}^{R T} \\
& 0 \leq R_{e v}^{u p^{s}} \leq R_{e v}^{u p^{\max }} \quad: Z_{e v, s, j}^{u p^{\max }}, Z_{e v, s, j}^{u p^{\min }} \quad \forall e v, s \\
& R_{e v}^{u p^{s}} \leq P O P_{e v} \\
& : Z_{e v, s, j}^{u p} \quad \forall e v, s \\
& 0 \leq R_{e v}^{d o w n^{s}} \leq R_{e v}^{d^{\text {max }}} \quad: Z_{e v, s, j}^{d^{\max }}, Z_{e v, s, j}^{d^{\text {min }}} \quad \forall e v, s \\
& P O P_{e v}+R_{e v}^{\text {down }} \leq P_{e v}^{\max } \quad: Z_{e v, s, j}^{\text {down }} \quad \forall e v, s \\
& 0 \leq R_{g}^{u p^{s}} \leq R_{g}^{u p^{\max }} \quad: Z_{g, s, j}^{u p^{\max }}, Z_{g, s, j}^{u p^{\min }} \quad \forall g, s \\
& P_{g}^{D A}+R_{g}^{u p^{s}} \leq R_{g}^{u p^{\max }} \quad: Z_{g, s, j}^{u p} \quad \forall g, s \\
& 0 \leq R_{g}^{\text {down }} \leq R_{g}^{d^{\text {max }}} \quad: Z_{g, s, j}^{d_{\text {max }}^{\text {max }}}, Z_{g, s, j}^{d^{\text {min }}} \quad \forall g, s
\end{aligned}
$$




$$
\begin{aligned}
& R_{g}^{\text {down }} \leq P_{g}^{D A} \\
& : Z_{g, s, j}^{\text {down }} \\
& : Z_{w, s, j}^{c^{\max }}, Z_{w, s, j}^{c^{\text {min }}} \quad \forall w, s \\
& 0 \leq L_{d}^{C^{s}} \leq L_{d}^{D A} \\
& : Z_{d, s, j}^{c^{\max }}, Z_{d, s, j}^{c^{\min }} \quad \forall d, s \\
& \mu_{e v, s}^{u p^{\max }}-\mu_{e v, s}^{u p^{m i n}}+\mu_{e v, s}^{u p}+\alpha_{e v}^{R T^{s}}-\rho_{s}^{R T}=0: Z R_{e v, j}^{u p^{s}} \\
& \mu_{e v, s}^{d^{\text {max }}}-\mu_{e v, s}^{d^{\text {min }}}+\mu_{e v, s}^{d o w n}-\alpha_{e v}^{R T^{s}}+\rho_{s}^{R T}=0 \quad: Z R_{e v, j}^{d^{s}} \\
& \mu_{g, s}^{u p^{\max }}-\mu_{g, s}^{u p^{\text {min }}}+\mu_{g, s}^{u p}+\alpha_{g}^{R T^{s}}-\rho_{s}^{R T}=0 \quad: Z R_{g, j}^{u p^{s}} \\
& \mu_{g, s}^{d^{\text {max }}}-\mu_{g, s}^{d^{\text {min }}}+\mu_{g, s}^{d o w n}-\alpha_{g}^{R T^{s}}+\rho_{s}^{R T}=0 \quad: Z R_{g, j}^{d^{s}} \\
& \mu_{w, t s}^{c^{\max }}-\mu_{w, s}^{c^{\min }}+\alpha_{w}^{R T^{s}}+\rho_{s}^{R T}=0 \\
& : Z P_{w, j}^{C^{s}} \\
& -\rho_{s}^{R T}+\mu_{d, s}^{c^{\max }}-\mu_{d, s}^{c^{m i n}}+C_{d}^{L}=0 \\
& : Z L_{d, j}^{C^{s}} \\
& \mu_{e v, s}^{u p^{\max }} \geq 0, \mu_{e v, s}^{u p^{\text {min }}} \geq 0 \\
& : X_{e v, s, j}^{u p^{\max }}, X_{e v, s, j}^{u p^{\min }} \quad \forall e v, s \\
& \mu_{e v, s}^{u p} \geq 0, \mu_{e v, s}^{\text {down }} \geq 0 \\
& : X_{e v, s, j}^{u p}, X_{e v, s, j}^{\text {down }} \quad \forall e v, s \\
& \mu_{e v, s}^{d^{\max }} \geq 0, \mu_{e v, s}^{d^{\text {min }}} \geq 0
\end{aligned}
$$

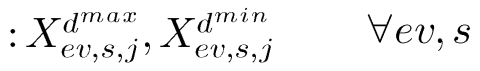

$$
\begin{aligned}
& \mu_{g, s}^{u p^{\max }} \geq 0, \mu_{g, s}^{u p^{m i n}} \geq 0 \\
& : X_{g, s, j}^{u p^{\max }}, X_{g, s, j}^{u p^{\min }} \quad \forall g, s \\
& \mu_{g, s}^{u p} \geq 0, \mu_{g, s}^{\text {down }} \geq 0 \\
& : X_{g, s, j}^{u p}, X_{g, s, j}^{\text {down }} \quad \forall g, s \\
& \mu_{g, s}^{d^{\max }} \geq 0, \mu_{g, s}^{d^{\text {min }}} \geq 0 \\
& : X_{g, s, j}^{d^{\max }}, X_{g, s, j}^{d^{\min }} \quad \forall g, s \\
& \mu_{w, s}^{c^{\max }} \geq 0, \mu_{w, s}^{c^{\min }} \geq 0
\end{aligned}
$$

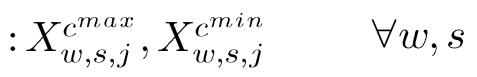

$$
\begin{aligned}
& \mu_{d, s}^{c^{\max }} \geq 0, \mu_{d, s}^{c^{\max }} \geq 0 \\
& : X_{d, s, j}^{c^{\max }}, X_{d, s, j}^{c^{\max }} \quad \forall d, s \\
& \sum_{w} P_{w}^{C^{s}} \cdot \alpha_{w}^{R T^{s}}+\sum_{d} C_{d}^{L} L_{d}^{C^{s}}+\sum_{e v} R_{e v}^{u p^{s}}-R_{e v}^{d o w n^{s}} \cdot \alpha_{e v}^{R T^{s}}+ \\
& \sum_{g}\left(R_{g}^{u p^{s}}-R_{g}^{d o w n^{s}}\right) \cdot \alpha_{g}^{R T^{s}}-\rho_{s}^{R T}\left(\sum_{w} P_{w}^{D A}-P_{w}^{R T^{s}}\right)+ \\
& \sum_{g} \mu_{g, s}^{u p^{\max }}\left(R_{g}^{u p^{\max }}\right)+\sum_{g} \mu_{g, s}^{u p}\left(R_{g}^{u p^{\max }}-P_{g}^{D A}\right)+
\end{aligned}
$$




$$
\begin{aligned}
& \sum_{g} \mu_{g, s}^{d^{\max }}\left(R_{g}^{d^{\max }}\right)+\sum_{g} \mu_{g, s}^{\text {down }}\left(P_{g}^{D A}\right)+\sum_{e v} \mu_{e v, s}^{u p^{\max }} R_{e v}^{u p^{\max }}+ \\
& \sum_{e v} \mu_{e v, s}^{u p} P O P_{e v}+\sum_{e v} R_{e v}^{d^{\max }} \mu_{e v, s}^{d^{\max }}+\sum_{e v} \mu_{e v, s}^{d}\left(P_{e v}^{\max }-\right. \\
& \left.P O P_{e v, t}\right)+\sum_{d} \mu_{d, s}^{c^{\max }} L_{d}^{D A}+\sum_{w} \mu_{w, s}^{c^{\max }} P_{w}^{R T^{s}}=0 \quad: \emptyset_{j, s}^{R T} \\
& \sum_{e v} E_{e v}^{D A}-\pi_{s} \sum_{s}\left[\sum_{e v} E e v,_{0}-1-N_{e v}^{s} \cdot D_{e v}^{s}+\sum_{e v} P O P_{e v}-\right. \\
& \left.R_{e v}^{u p^{s}}+R_{e v}^{d o w n^{s}}\right]=0 \quad: \delta_{j}^{T} \\
& E_{e v}^{D A}=\pi_{s} \sum_{s} \sum_{i} E_{e v}^{s} \quad \forall e v \\
& E_{e v}^{M i n} \leq E_{e v}^{D A} \leq E_{e v}^{M a x} \quad: Z_{e v, s, j}^{E E V^{\max }}, Z_{e v, s, j}^{E E V^{\text {min }}} \forall e v \\
& \rho^{T}-\rho^{D A}+\mu_{e v}^{E E V^{\max }}-\mu_{e v}^{E E V^{m i n}}=0 \quad: Z E_{e v, j}^{D A} \quad \forall e v \\
& \mu_{e v}^{E E V^{\max }} \geq 0, \mu_{e v}^{E E V^{m i n}} \geq 0 \quad: X_{e v, j}^{E E V^{\max }}, X_{e v, j}^{E E V^{m i 1}} \forall e v \\
& -\sum_{e v, t} \rho^{D A} E_{e v}^{D A}+\sum_{t} \mu_{e v}^{E E V^{\max }} E_{e v}^{M a x}- \\
& \sum_{t} \mu_{e v}^{E E V^{m i n}} E_{e v}^{M i n}+\rho^{T} \cdot \pi_{s} \sum_{s}\left[\sum_{e v} E_{e v}^{D A}-\sum_{e v} E e v,_{0}+\right. \\
& \left.1-N_{e v}^{s} \cdot D_{e v}^{s}-\sum_{e v} P O P_{e v}-R_{e v}^{u p^{s}}+R_{e v}^{d o w n^{s}}\right]=0 \quad: \emptyset_{j}^{E V}
\end{aligned}
$$

\subsubsection{Equilibrium Problem with Equilibrium Constraints}

The joint solution of the MPECs of all firms (5.5a)—(5.7a) constitutes an EPEC, which is illustrated in Fig. 1.3, Chapter 1. The EPEC solution determines the market equilibria associated with their corresponding KKT conditions. The formulation is as follows:

The equality constraints of MPECs of all firms (5.5a)—(5.7d) are as follows:

$$
\begin{aligned}
& (5.5 \mathrm{~h}),(5.5 \mathrm{~m})-(5.5 \mathrm{p}),(5.5 \mathrm{u}),(5.6 \mathrm{~g})-(5.6 \mathrm{l}),(5.6 \mathrm{u}),(5.6 \mathrm{v}), \\
& (5.6 \mathrm{w}),(5.6 \mathrm{y}),(5.7 \mathrm{a})
\end{aligned} \quad \forall j
$$

The equality constraints derived from the Lagrangian of all firms MPECs (5.5a) - (5.7a) with respect to variables are as follows: 


$$
\begin{aligned}
& \frac{\partial L}{\partial P O P_{e v}}=\rho^{D A}-\rho^{T}-\delta_{j}^{D A}+Z_{e v, j}^{D A^{\max }}-Z_{e v, j}^{D A^{\min }}-\emptyset_{j}^{D A} \beta_{e v}^{D A} \\
& +\sum_{s}\left(\left(-Z_{e v, s, j}^{u p}+Z_{e v, s, j}^{d o w n}\right.\right. \\
& \left.\left.+\emptyset_{j, s}^{R T} \cdot\left(\mu_{e v, s}^{u p}-\mu_{e v, s}^{d}\right)-\pi_{s} \delta_{j}^{T}+\pi_{s} \rho^{T} \emptyset_{j}^{E V}\right)\right) \\
& =0 \\
& \forall e v, j \in \mathrm{Y}_{j} \\
& \frac{\partial L}{\partial P O P_{e v}}=-\delta_{j}^{D A}+Z_{e v, j}^{D A^{\max }}-Z_{e v, j}^{D A^{\min }}-\emptyset_{j}^{D A} \beta_{e v}^{D A} \\
& +\sum_{s}\left(\left(-Z_{e v, s, j}^{u p}+Z_{e v, s, j}^{\text {down }}\right.\right. \\
& \left.\left.+\emptyset_{j, s}^{R T} \cdot\left(\mu_{e v, s}^{u p}-\mu_{e v, s}^{d}\right)-\pi_{s} \delta_{j}^{T}+\pi_{s} \rho^{T} \emptyset_{j}^{E V}\right)\right) \\
& =0 \quad \forall e v, j \notin \mathrm{Y}_{j} \\
& \frac{\partial L}{\partial P_{g}^{D A}}=-\rho^{D A}+C_{g}+\delta_{j}^{D A}+Z_{g, j}^{D A^{\max }}-Z_{g, j}^{D A^{\min }}+\emptyset_{j}^{D A} \alpha_{g}^{D A} \\
& +\sum_{s}\left(\left(Z_{g, s, j}^{u p}-Z_{g, s, j}^{\text {down }}\right.\right. \\
& \left.\left.+\emptyset_{j, s}^{R T} \cdot\left(\mu_{g, s}^{d o w n}-\mu_{g, s}^{u p}\right)\right)\right)=0 \\
& \forall g, j \in \mathrm{Y}_{j} \\
& \frac{\partial L}{\partial P_{g}^{D A}}=+\delta_{j}^{D A}+Z_{g, j}^{D A^{\max }}-Z_{g, j}^{D A^{\min }}+\emptyset_{j}^{D A} \alpha_{g}^{D A} \\
& +\sum_{s}\left(\left(Z_{g, s, j}^{u p}-Z_{g, s, j}^{d o w n}\right.\right. \\
& \left.\left.+\emptyset_{j, s}^{R T} \cdot\left(\mu_{g, s}^{d o w n}-\mu_{g, s}^{u p}\right)\right)\right)=0 \\
& \forall g, j \notin \mathrm{Y}_{j} \\
& \frac{\partial L}{\partial P_{w}^{D A}}=-\rho^{D A}+\delta_{j}^{D A}+Z_{w, j}^{D A^{\max }}-Z_{w, j}^{D A^{\min }}+\emptyset_{j}^{D A} \alpha_{w}{ }_{w} A \\
& +\sum_{s}\left(\pi_{s} \cdot \rho_{s}^{R T}+\delta_{j, s}^{R T}+\emptyset_{j, s}^{R T} \rho_{s}^{R T}\right)=0 \quad \forall w, j \in \mathrm{Y}_{j} \\
& \frac{\partial L}{\partial P_{w}^{D A}}=+\delta_{j}^{D A}+Z_{w, j}^{D A^{\max }}-Z_{w, j}^{D A^{\min }}+\emptyset_{j}^{D A} \alpha_{w}^{D A} \\
& +\sum_{s}\left(\pi_{s} \cdot \rho_{s}^{R T}+\delta_{j, s}^{R T}+\emptyset_{j, s}^{R T} \rho_{s}^{R T}\right)=0 \quad \forall w, j \notin \mathrm{Y}_{j} \\
& \frac{\partial L}{\partial L_{d}^{D A}}=-\delta_{j}^{D A}+Z_{d, j}^{D A^{\max }}-Z_{d, j}^{D A^{\min }}-\emptyset_{j}^{D A} B_{d}^{D A} \\
& +\sum_{s}\left(\emptyset_{j, s}^{R T} \mu_{d, s}^{c^{\max }}-Z_{d, s, j}^{c^{\max }}\right)=0
\end{aligned}
$$




$$
\begin{aligned}
& \frac{\partial L}{\partial R_{e v}^{u p^{s}}}=-\pi_{s}\left(\rho_{s}^{R T}-\rho^{T}\right)-\delta_{j, s}^{R T}+Z_{e v, s, j}^{u p^{\max }}-Z_{e v, s, j}^{u p^{\min }}+Z_{e v, s, j}^{u p} \\
& +\emptyset_{j, s}^{R T} \alpha_{e v}^{R T^{s}}+\pi_{s} \delta_{j}^{T}-\pi_{s} \rho^{T} \emptyset_{j}^{E V}=0 \quad \forall s, j, e v \in \mathrm{Y}_{j} \\
& \frac{\partial L}{\partial R_{e v}^{u p^{s}}}=-\delta_{j, s}^{R T}+Z_{e v, s, j}^{u p^{\max }}-Z_{e v, s, j}^{u p^{m i n}}+Z_{e v, s, j}^{u p}+\emptyset_{j, s}^{R T} \alpha_{e v}^{R T^{s}}+\pi_{s} \delta_{j}^{T} \\
& -\pi_{s} \rho^{T} \emptyset_{j}^{E V}=0 \\
& \frac{\partial L}{\partial R_{e v}^{d o w n^{s}}}=\pi_{s}\left(\rho_{s}^{R T}-\rho^{T}\right)+\delta_{j, s}^{R T}+Z_{e v, s, j}^{d^{\max }}-Z_{e v, s, j}^{d^{\min }}+Z_{e v, s, j}^{d o w n} \\
& -\emptyset_{j, s}^{R T} \alpha_{g}^{R T^{s}}-\pi_{s} \delta_{j}^{T}+\pi_{s} \rho^{T} \emptyset_{j}^{E V}=0 \quad \forall s, j, e v \in \mathrm{Y}_{j} \\
& \frac{\partial L}{\partial R_{e v}^{d o w n}}=+\delta_{j, s}^{R T}+Z_{e v, s, j}^{d^{m a x}}-Z_{e v, s, j}^{d^{m i n}}+Z_{e v, s, j}^{d o w n}-\emptyset_{j, s}^{R T} \alpha_{g}^{R T^{s}} \\
& -\pi_{s} \delta_{j}^{T}+\pi_{s} \rho^{T} \emptyset_{j}^{E V}=0 \quad \forall s, j, e v \notin \mathrm{Y}_{j} \\
& \frac{\partial L}{\partial R_{g}^{u p^{s}}}=-\pi_{s}\left(\rho_{s}^{R T}-C_{g}\right)-\delta_{j, s}^{R T}+Z_{g, s, j}^{u p^{\max }}-Z_{g, s, j}^{u p^{\min }}+Z_{g, s, j}^{u p} \\
& +\emptyset_{j, s}^{R T} \alpha_{g}^{R T^{s}}=0 \\
& \frac{\partial L}{\partial R_{g}^{u p^{s}}}=-\delta_{j, s}^{R T}+Z_{g, s, j}^{u p^{\max }}-Z_{g, s, j}^{u p^{\min }}+Z_{g, s, j}^{u p}+\emptyset_{j, s}^{R T} \alpha_{g}^{R T^{s}}=0 \\
& \frac{\partial L}{\partial R_{g}^{d o w n^{s}}}=\pi_{s}\left(\rho_{s}^{R T}-C_{g}\right)+\delta_{j, s}^{R T}+Z_{g, s, j}^{d^{m a x}}-Z_{g, s, j}^{d^{m i n}}+Z_{g, s, j}^{d o w n} \\
& -\emptyset_{j, s}^{R T} \alpha_{g}^{R T^{s}}=0 \\
& \forall s, j, g \in \mathrm{Y}_{j} \\
& \frac{\partial L}{\partial R_{g}^{d o w n}}=+\delta_{j, s}^{R T}+Z_{g, s, j}^{d^{m a x}}-Z_{g, s, j}^{d^{m i n}}+Z_{g, s, j}^{d o w n}-\emptyset_{j, s}^{R T} \alpha_{g}^{R T^{s}}=0 \quad \forall s, j, g \notin \mathrm{Y}_{j} \\
& \frac{\partial L}{\partial L_{d}^{C^{s}}}=-\delta_{j, s}^{R T}+Z_{d, s, j}^{c^{\max }}-Z_{d, s, j}^{\min ^{m}}+\emptyset_{j, s}^{R T} C_{d}^{L}=0 \\
& \frac{\partial L}{\partial P_{w, s}^{S}}=\pi_{s} \rho_{s}^{R T}+\delta_{j, s}^{R T}+Z_{w, s, j}^{c^{\max }}-Z_{w, s, j}^{c^{m i n}}+\emptyset_{j, s}^{R T} \alpha_{w}^{R T^{s}}=0 \\
& \frac{\partial L}{\partial P_{w, s}^{S}}=+\delta_{j, s}^{R T}+Z_{w, s, j}^{c^{\max }}-Z_{w, s, j}^{c^{m i n}}+\emptyset_{j, s}^{R T} \alpha_{w}^{R T^{s}}=0 \\
& \frac{\partial L}{\partial \beta_{e v}^{D A}}=-Z \beta_{e v, j}^{D A}-Z P O P_{e v, j}-\emptyset_{j}^{D A} P O P_{e v}=0 \\
& \forall s, j, e v \notin \mathrm{Y}_{j} \\
& \forall s, j, g \in \mathrm{Y}_{j} \\
& \forall s, j, d \\
& \forall s, j, w \in \mathrm{Y}_{j} \\
& \forall s, j, w \notin \mathrm{Y}_{j}
\end{aligned}
$$




$$
\begin{aligned}
& \frac{\partial L}{\partial \beta_{e v}^{D A}}=-Z P O P_{e v, j}-\emptyset_{j}^{D A} P O P_{e v}=0 \\
& \frac{\partial L}{\partial \alpha_{g}^{D A}}=-Z \alpha_{g, j}^{D A}+Z P_{g, j}^{D A}+\emptyset_{j}^{D A} P_{g}^{D A}=0 \\
& \frac{\partial L}{\partial \alpha_{g}^{D A}}=+Z P_{g, j}^{D A}+\emptyset_{j}^{D A} P_{g}^{D A}=0 \\
& \frac{\partial L}{\partial \alpha_{w}^{D A}}=-Z \alpha_{w, j}^{D A}+Z P_{w, j}^{D A}+\emptyset_{j}^{D A} P_{w}^{D A}=0 \\
& \frac{\partial L}{\partial \alpha_{w}^{D A}}=+Z P_{w, j}^{D A}+\emptyset_{j}^{D A} P_{w}^{D A}=0 \\
& \frac{\partial L}{\partial \alpha_{e v}^{R T^{s}}}=-Z \alpha_{e v, j}^{R T^{s}}+Z R_{e v, j}^{u p^{s}}-Z R_{e v, j}^{d^{s}}+\emptyset_{j, s}^{R T}\left(R_{e v}^{u p^{s}}-R_{e v}^{d o w n^{s}}\right) \\
& =0 \\
& \frac{\partial L}{\partial \alpha_{e v}^{R T^{s}}}+Z R_{e v, j}^{u p^{s}}-Z R_{e v, j}^{d^{s}}+\emptyset_{j, s}^{R T}\left(R_{e v}^{u p^{s}}-R_{e v}^{d o w n^{s}}\right)=0 \\
& \frac{\partial L}{\partial \alpha_{g}^{R T^{s}}}=-Z \alpha_{g, j}^{R T^{s}}+Z R_{g, j}^{u p^{s}}-Z R_{g, j}^{d^{s}}+\emptyset_{j, s}^{R T}\left(R_{g}^{u p^{s}}-R_{g}^{d o w n^{s}}\right) \\
& =0 \\
& \forall s, j, g \in \mathrm{Y}_{j} \\
& \frac{\partial L}{\partial \alpha_{g}^{R T^{s}}}=+Z R_{g, j}^{u p^{s}}-Z R_{g, j}^{d^{s}}+\emptyset_{j, s}^{R T}\left(R_{g}^{u p^{s}}-R_{g}^{d o w n^{s}}\right)=0 \\
& \frac{\partial L}{\partial \alpha_{w}^{R T^{s}}}=-Z \alpha_{w, j}^{R T^{s}}+Z P_{w, j}^{C^{s}}+\emptyset_{j, s}^{R T} P_{w}^{C^{s}}=0 \\
& \frac{\partial L}{\partial \alpha_{w}^{R T^{s}}}=+Z P_{w, j}^{C^{\varsigma}}+\emptyset_{j, s}^{R T} P_{w}^{C^{\varsigma}}=0 \\
& \frac{\partial L}{\partial \rho^{D A}}=-\sum_{g \in \Omega_{j}} P_{g}^{D A}-\sum_{w \in \Omega_{j}} P_{w}^{D A}+\sum_{e v \in \Omega_{j}} P O P_{e v}+Z P O P_{e v, j} \\
& -\sum_{g} Z P_{g, j}^{D A}-\sum_{w} Z P_{w, j}^{D A}+\sum_{d} Z L_{d, j}^{D A} \\
& -Z E_{e v, j}^{D A}-\emptyset_{j}^{E V} E_{e v}^{D A}=0 \\
& \forall j, e v \notin \mathrm{Y}_{j} \\
& \forall j, g \in \mathrm{Y}_{j} \\
& \forall j, g \notin \mathrm{Y}_{j} \\
& \forall j, w \in \mathrm{Y}_{j} \\
& \forall j, w \notin \mathrm{Y}_{j} \\
& \forall s, j, e v \in \mathrm{Y}_{j} \\
& \forall s, j, e v \notin \mathrm{Y}_{j} \\
& \forall s, j, g \notin \mathrm{Y}_{j} \\
& \forall s, j, w \in \mathrm{Y}_{j} \\
& \forall s, j, w \notin \mathrm{Y}_{j}
\end{aligned}
$$




$$
\begin{aligned}
& \frac{\partial L}{\partial \rho_{s}^{R T}}=-\pi_{s} \cdot \sum_{g \in \Omega_{j}}\left(R_{g, s}^{u p}-R_{g, s}^{d}\right) \\
& +\pi_{s} \cdot \sum_{w \in \Omega_{j}}\left(P_{w}^{D A}+P_{w, s}^{S}-P_{w, s}^{R T}\right)+ \\
& -\pi_{s} \cdot \sum_{e v \in \Omega_{j}}\left(R_{e v, s}^{u p}-R_{e v, s}^{d}\right) \\
& +\sum_{e v}\left(Z R_{e v, j}^{d^{s}}-Z R_{e v, j}^{u p^{s}}\right) \\
& +\sum_{g}\left(Z R_{g, j}^{d^{s}}-Z R_{g, j}^{u p^{s}}\right)+\sum_{w} Z P_{w, j}^{C^{s}} \\
& -\sum_{d} Z L_{d, j}^{C^{s}}-\sum_{w} \emptyset_{j, s}^{R T}\left(P_{w}^{D A}-P_{w}^{R T^{\delta}}\right)=0 \quad \forall j, s \\
& \frac{\partial L}{\partial E_{e v}^{D A}}=+\delta_{j}^{T}+Z_{e v, s, j}^{E E V^{\max }}-Z_{e v, s, j}^{E E V^{\min }}-\emptyset_{j}^{E V} \rho^{D A}+\emptyset_{j}^{E V} \rho^{T}=0 \quad \forall j, g \\
& \frac{\partial L}{\partial \rho^{T}}=-P O P_{e v}+R_{e v}^{u s^{s}}-R_{e v}^{d o w n^{s}}+Z E_{e v, j}^{D A} \\
& +\emptyset_{j}^{E V} \pi_{s} \sum_{s}\left[\sum_{e v} E_{e v},_{0}-1-N_{e v}^{s} . D_{e v}^{s}\right. \\
& \left.+\sum_{e v}\left(P O P_{e v}-R_{e v}^{u p^{s}}+R_{e v}^{d o w n^{s}}\right)\right]=0 \quad \forall j, g \\
& \frac{\partial L}{\partial \mu_{e v}^{D A^{m i n}}}=-Z P O P_{e v, j}-X_{e v, j}^{D A^{m i n}}=0 \\
& \frac{\partial L}{\partial \mu_{e v}^{D A^{\max }}}=Z P O P_{e v, j}-X_{e v, j}^{D A^{\max }}+\emptyset_{j}^{D A} P_{e v}^{\max }=0 \\
& \frac{\partial L}{\partial \mu_{e v}^{E E V^{\min }}}=-Z E_{e v, j}^{D A}-X_{e v, j}^{E E V^{\min }}-\emptyset_{j}^{E V} E_{e v}^{M i n}=0 \\
& \frac{\partial L}{\partial \mu_{e v}^{E E V^{\max }}}=Z E_{e v, j}^{D A}-X_{e v, j}^{E E V^{\max }}+\emptyset_{j}^{E V} E_{e v}^{M a x}=0 \\
& \frac{\partial L}{\partial \mu_{g}^{D A^{\min }}}=-Z P_{g, j}^{D A}-X_{g, j}^{D A^{\min }}=0 \\
& \frac{\partial L}{\partial \mu_{g}^{D A^{\max }}}=Z P_{g, j}^{D A}-X_{g, j}^{D A^{\max }}+\emptyset_{j}^{D A} P_{g}^{\max }=0 \\
& \frac{\partial L}{\partial \mu_{w}^{D A^{\min }}}=-Z P_{w, j}^{D A}-X_{w, j}^{D A^{\min }}=0
\end{aligned}
$$




$$
\begin{aligned}
& \frac{\partial L}{\partial \mu_{w}^{D A^{\max }}}=Z P_{w, j}^{D A}-X_{w, j}^{D A^{\max }}+\emptyset_{j}^{D A} P_{w}^{\max }=0 \\
& \frac{\partial L}{\partial \mu_{d}^{D A^{\min }}}=-Z L_{d, j}^{D A}-X_{d, j}^{D A^{\min }}=0 \\
& \frac{\partial L}{\partial \mu_{d}^{D A^{\max }}}=Z L_{d, j}^{D A}-X_{d, j}^{D A^{\max }}+\emptyset_{j}^{D A} L_{d}^{\max }=0 \\
& \frac{\partial L}{\partial \mu_{g, s}^{u p^{m i n}}}=-Z R_{g, j}^{u p^{s}}-X_{g, s, j}^{u p^{m i n}}=0 \\
& \frac{\partial L}{\partial \mu_{g, s}^{u p^{\max }}}=Z R_{g, j}^{u p^{s}}-X_{g, s, j}^{u p^{\max }}+\emptyset_{j, s}^{R T} R_{g}^{u p^{\max }}=0 \\
& \frac{\partial L}{\partial \mu_{g, s}^{d^{m i n}}}=-Z R_{g, j}^{d^{s}}-X_{g, s, j}^{d^{\min }}=0 \\
& \frac{\partial L}{\partial \mu_{g, s}^{d^{\max }}}=Z R_{g, j}^{d^{s}}-X_{g, s, j}^{d^{\max }}+\emptyset_{j, s}^{R T} R_{g}^{d^{\max }}=0 \\
& \frac{\partial L}{\partial \mu_{g, s}^{\text {down }}}=Z R_{g, j}^{d^{s}}-X_{g, s, j}^{d o w n}+\emptyset_{j, s}^{R T} P_{g}^{D A}=0 \\
& \frac{\partial L}{\partial \mu_{g, s}^{u p}}=Z R_{g, j}^{u p^{s}}-X_{g, s, j}^{u p}+\emptyset_{j, s}^{R T}\left(P_{g}^{\max }-P_{g}^{D A}\right)=0 \\
& \frac{\partial L}{\partial \mu_{e v, s}^{u p^{m i n}}}=-Z R_{e v, j}^{u p^{s}}-X_{e v, s, j}^{u p^{m i n}}=0 \\
& \frac{\partial L}{\partial \mu_{e v, s}^{u p^{\max }}}=Z R_{e v, j}^{u p^{s}}-X_{e v, s, j}^{u p^{\max }}+\emptyset_{j, s}^{R T} R_{e v}^{u p^{\max }}=0 \\
& \frac{\partial L}{\partial \mu_{g, s}^{d^{m i n}}}=-Z R_{e v, j}^{d^{s}}-X_{e v, s, j}^{d^{\min }}=0 \\
& \frac{\partial L}{\partial \mu_{e v, s}^{d^{\max }}}=Z R_{e v, j}^{d^{s}}-X_{e v, s, j}^{d^{\max }}+\emptyset_{j, s}^{R T} R_{g e v}^{d^{\max }}=0 \\
& \frac{\partial L}{\partial \mu_{e v, s}^{d o w n}}=Z R_{e v, j}^{d^{s}}-X_{e v, s, j}^{d o w n}+\emptyset_{j, s}^{R T} P_{e v}^{\max }-P O P_{e v}=0 \\
& \forall j, w \\
& \forall j, d \\
& \forall j, d \\
& \forall j, g, s
\end{aligned}
$$




$$
\begin{array}{ll}
\frac{\partial L}{\partial \mu_{e v, s}^{u p}}=Z R_{e v, j}^{u p^{s}}-X_{e v, s, j}^{u p}+\emptyset_{j, s}^{R T} P O P_{e v}=0 & \forall j, e v, s \\
\frac{\partial L}{\partial \mu_{d, s}^{c^{m i n}}}=-Z L_{d, s, j}^{c}-X_{w, s, j}^{c^{m i n}}=0 & \forall j, d, s \\
\frac{\partial L}{\partial \mu_{d, s}^{c^{m a x}}}=Z L_{d, s, j}^{c}-X_{w, s, j}^{c^{\max }}+\emptyset_{j, s}^{R T} L_{d}=0 & \forall j, d, s \\
\frac{\partial L}{\partial \mu_{w, s}^{c^{m i n}}}=-Z P_{w, j}^{C^{s}}-X_{w, s, j}^{c^{m i n}}=0 & \\
\frac{\partial L}{\partial \mu_{w, t s}^{c^{m a x}}}=Z P_{w, j}^{C^{s}}-X_{w, s, j}^{c^{m a x}}+\emptyset_{j, s}^{R T} P_{w}^{R T^{s}}=0 & \forall j, w, s \\
& \forall j, w, s
\end{array}
$$

The complementarity conditions of all firms' MPECs (5.5a)-(5.7a) are as follows:

$$
\begin{array}{ll}
0 \leq \alpha_{g}^{D A} \perp Z \alpha_{g}^{D A} \geq 0 & \forall j, g \in \mathrm{Y}_{j} \\
0 \leq \alpha_{w}^{D A} \perp Z \alpha_{w}^{D A} \geq 0 & \forall j, w \in \mathrm{Y}_{j} \\
0 \leq \beta_{e v}^{D A} \perp Z \beta_{e v}^{D A} \geq 0 & \forall j, e v \in \mathrm{Y}_{j} \\
0 \leq \alpha_{g}^{R T^{s}} \perp Z \alpha_{g}^{R T^{s}} \geq 0 & \forall j, g \in \mathrm{Y}_{j}, \forall s \\
0 \leq \alpha_{w}^{R T^{s}} \perp Z \alpha_{w}^{R T^{s}} \geq 0 & \forall j, w \in \mathrm{Y}_{j}, \forall s \\
0 \leq \alpha_{e v}^{R T^{s}} \perp Z \alpha_{e v}^{R T^{s}} \geq 0 & \forall j, e v \in \mathrm{Y}_{j}, \forall s \\
0 \leq P_{e v}^{\max }-P O P_{e v} \perp Z_{e v, j}^{D A^{\text {max }}} \geq 0 & \forall j, e v \\
0 \leq P_{g}^{\text {max }}-P_{g}^{D A} \perp Z_{g, j}^{D A^{\text {max }}} \geq 0 & \\
0 \leq P_{w}^{\text {max }}-P_{w}^{D A} \perp Z_{w, j}^{D A^{\text {max }}} \geq 0 & \forall j, g
\end{array}
$$




$$
\begin{aligned}
& 0 \leq L_{d}^{\max }-L_{d}^{D A} \perp Z_{d, j}^{D A^{\max }} \geq 0 \quad \forall j, d \\
& 0 \leq P O P_{e v} \perp Z_{e v, j}^{D A^{m i n}} \geq 0 \quad \forall j, e v \\
& 0 \leq P_{g}^{D A} \perp Z_{g, j}^{D A^{m i n}} \geq 0 \quad \forall j, g \\
& 0 \leq P_{w}^{D A} \perp Z_{w, j}^{D A^{m i n}} \geq 0 \quad \forall j, w \\
& 0 \leq L_{d}^{D A} \perp Z_{d, j}^{D A^{m i n}} \geq 0 \quad \forall j, d \\
& 0 \leq \mu_{e v}^{D A^{\max }} \perp X_{e v, j}^{D A^{\max }} \geq 0 \quad \forall j, e v \\
& 0 \leq \mu_{g}^{D A^{\max }} \perp X_{g, j}^{D A^{\max }} \geq 0 \quad \forall j, g \\
& 0 \leq \mu_{w}^{D A^{\max }} \perp X_{w, j}^{D A^{\max }} \geq 0 \quad \forall j, w \\
& 0 \leq \mu_{d}^{D A^{\max }} \perp X_{d, j}^{D A^{\max }} \geq 0 \quad \forall j, d \\
& 0 \leq \mu_{e v}^{D A^{m i n}} \perp X_{e v, j}^{D A^{m i n}} \geq 0 \quad \forall j, e v \\
& 0 \leq \mu_{g}^{D A^{m i n}} \perp X_{g, j}^{D A^{m i n}} \geq 0 \quad \forall j, g \\
& 0 \leq \mu_{w}^{D A^{m i n}} \perp X_{w, j}^{D A^{m i n}} \geq 0 \quad \forall j, w \\
& 0 \leq \mu_{d}^{D A^{m i n}} \perp X_{d, j}^{D A^{m i n}} \geq 0 \quad \forall j, d \\
& 0 \leq R_{e v}^{u p^{\max }}-R_{e v}^{u p^{s}} \perp Z_{e v, s, j}^{u p^{\max }} \geq 0 \quad \forall j, e v, s \\
& 0 \leq P O P_{e v}-R_{e v}^{u p^{s}} \perp Z_{e v, s, j}^{u p} \geq 0 \quad \forall j, e v, s
\end{aligned}
$$




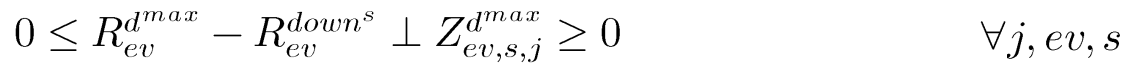

$$
\begin{aligned}
& 0 \leq P_{e v}^{\max }-P O P_{e v}-R_{e v}^{d o w n^{s}} \perp Z_{e v, s, j}^{\text {down }} \geq 0 \quad \forall j, e v, s \\
& 0 \leq R_{g}^{u p^{\max }}-R_{g}^{u p^{s}} \perp Z_{g, s, j}^{u p^{\max }} \geq 0 \quad \forall j, g, s \\
& 0 \leq R_{g}^{u p^{\max }}-P_{g}^{D A}-R_{g}^{u p^{s}} \perp Z_{g, s, j}^{u p} \geq 0 \quad \forall j, g, s \\
& 0 \leq R_{g}^{d^{\max }}-R_{g}^{\text {down }^{s}} \perp Z_{g, s, j}^{d^{\max }} \geq 0 \quad \forall j, g, s \\
& 0 \leq P_{g}^{D A}-R_{g}^{\text {down }}{ }^{s} \perp Z_{g, s, j}^{\text {down }} \geq 0 \quad \forall j, g, s \\
& 0 \leq P_{w}^{R T^{s}}-P_{w}^{C^{s}} \perp Z_{w, s, j}^{c^{\max }} \geq 0 \quad \forall j, w, s \\
& 0 \leq L_{d}^{\max }-L_{d}^{C^{s}} \perp Z_{d, s, j}^{c^{\max }} \geq 0 \quad \forall j, d, s \\
& 0 \leq R_{e v}^{u p^{s}} \perp Z_{e v, s, j}^{u p^{m i n}} \geq 0 \quad \forall j, e v, s \\
& 0 \leq R_{e v}^{d o w n^{s}} \perp Z_{e v, s, j}^{d^{m i n}} \geq 0 \quad \forall j, e v, s \\
& 0 \leq R_{g}^{u p^{s}} \perp Z_{g, s, j}^{u p^{m i n}} \geq 0 \quad \forall j, g, s \\
& 0 \leq R_{g}^{\text {down }} \perp_{g, s, j}^{d^{\text {min }}} \geq 0 \quad \forall j, g, s \\
& 0 \leq P_{w}^{C^{s}} \perp Z_{w, s, j}^{c^{m i n}} \geq 0 \quad \forall j, w, s \\
& 0 \leq L_{d}^{C^{s}} \perp Z_{d, s, j}^{c^{m i n}} \geq 0 \quad \forall j, d, s \\
& 0 \leq R_{e v}^{u p^{\max }}-R_{e v}^{u p^{s}} \perp Z_{e v, s, j}^{u p^{\max }} \geq 0 \quad \forall j, e v, s
\end{aligned}
$$




$$
\begin{aligned}
& 0 \leq P O P_{e v}-R_{e v}^{u p^{s}} \perp Z_{e v, s, j}^{u p} \geq 0 \quad \forall j, e v, s \\
& 0 \leq R_{e v}^{d^{\text {max }}}-R_{e v}^{\text {down }}{ }^{s} \perp Z_{e v, s, j}^{d^{\max }} \geq 0 \quad \forall j, e v, s \\
& 0 \leq P_{e v}^{\max }-P O P_{e v}-R_{e v}^{\text {down }}{ }^{s} \perp Z_{e v, s, j}^{\text {down }} \geq 0 \quad \forall j, e v, s \\
& 0 \leq \mu_{g, s}^{u p^{\max }} \perp X_{g, s, j}^{u p^{\max }} \geq 0 \quad \forall j, g, s \\
& 0 \leq \mu_{g, s}^{u p} \perp X_{g, s, j}^{u p} \geq 0 \quad \forall j, g, s \\
& 0 \leq \mu_{g, s}^{d^{\max }} \perp X_{g, s, j}^{d^{\max }} \geq 0 \quad \forall j, g, s \\
& 0 \leq \mu_{g, s}^{\text {down }} \perp X_{g, s, j}^{\text {down }} \geq 0 \quad \forall j, g, s \\
& 0 \leq \mu_{w, s}^{c^{\max }} \perp X_{w, s, j}^{c^{\max }} \geq 0 \quad \forall j, w, s \\
& 0 \leq \mu_{d, s}^{c^{\max }} \perp X_{d, s, j}^{c^{\max }} \geq 0 \quad \forall j, d, s \\
& 0 \leq \mu_{e v, s}^{u p^{m i n}} \perp X_{e v, s, j}^{u p^{m i n}} \geq 0 \quad \forall j, e v, s \\
& 0 \leq \mu_{e v, s}^{d^{m i n}} \perp X_{e v, s, j}^{d^{m i n}} \geq 0 \quad \forall j, e v, s \\
& 0 \leq \mu_{g, s}^{u p^{m i n}} \perp X_{g, s, j}^{u p^{m i n}} \geq 0 \quad \forall j, g, s \\
& 0 \leq \mu_{g, s}^{d^{\text {min }}} \perp X_{g, s, j}^{d^{\text {min }}} \geq 0 \quad \forall j, g, s \\
& 0 \leq \mu_{w, s}^{c^{m i n}} \perp X_{w, s, j}^{c^{m i n}} \geq 0 \quad \forall j, w, s \\
& 0 \leq \mu_{d, s}^{c^{m i n}} \perp X_{d, s, j}^{c^{m i n}} \geq 0 \quad \forall j, d, s
\end{aligned}
$$




$$
\begin{array}{ll}
0 \leq E_{e v}^{D A}-E_{e v}^{M a x} \perp Z_{e v, j}^{E E V^{\max }} \geq 0 & \forall j, e v \\
0 \leq E_{e v}^{M i n}-E_{e v}^{D A} \perp Z_{e v, j}^{E E V^{\text {min }}} \geq 0 & \forall j, e v \\
0 \leq \mu_{e v}^{E E V^{\max }} \perp X_{e v, j}^{E E V^{\max }} \geq 0 & \forall j, e v \\
0 \leq \mu_{e v}^{E E V^{\text {min }}} \perp X_{e v, j}^{E E V^{\text {min }}} \geq 0 & \\
& \forall j, e v
\end{array}
$$

\subsubsection{Mixed-Integer Linear Programming}

The EPEC (5.8)-(5.14f) are converted to a MILP by linearizing of three nonlinearities including the nonlinear terms in the strong duality equalities, the complementarity conditions, and the nonlinear terms comprising $\emptyset_{j}^{E V}, \emptyset_{j, s}^{R T}$, and $\emptyset_{j}^{D A}$.

\subsubsection{Linearization of Nonlinear Terms in the Strong Duality Equalities}

Nonlinear terms in the strong duality equality $(5.5 \mathrm{u})$ are replaced by the complementarity conditions as follows:

$$
\begin{array}{ll}
0 \leq P_{e v}^{\max }-P O P_{e v} \perp \mu_{e v}^{D A^{\max }} \geq 0 & \forall e v \\
0 \leq P_{g}^{\max }-P_{g}^{D A} \perp \mu_{g}^{D A^{\max } \geq 0} & \forall g \\
0 \leq P_{w}^{\max }-P_{w}^{D A} \perp \mu_{w}^{D A^{\max }} \geq 0 & \forall w \\
0 \leq L_{d}^{\max }-L_{d}^{D A} \perp \mu_{d}^{D A^{\max }} \geq 0 & \forall d
\end{array}
$$




$$
\begin{array}{ll}
0 \leq P O P_{e v} \perp \mu_{e v}^{D A^{m i n}} \geq 0 & \forall e v \\
0 \leq P_{g, t}^{D A} \perp \mu_{g}^{D A^{m i n}} \geq 0 & \forall g \\
0 \leq P_{w}^{D A} \perp \mu_{w}^{D A^{m i n}} \geq 0 & \forall w \\
0 \leq L_{d}^{D A} \perp \mu_{d}^{D A^{m i n}} \geq 0 &
\end{array}
$$

Nonlinear terms in the strong duality equality (5.6u) are replaced by the complementarity conditions as follows:

$$
\begin{aligned}
& 0 \leq R_{e v}^{u p^{\max }}-R_{e v}^{u p^{s}} \perp \mu_{e v, s}^{u p^{\max }} \geq 0 \\
& \forall e v, s \\
& 0 \leq P O P_{e v}-R_{e v}^{u p^{s}} \perp \mu_{e v, s}^{u p} \geq 0 \quad \forall e v, s \\
& 0 \leq R_{e v}^{d^{\max }}-R_{e v}^{\text {down }}{ }^{s} \perp \mu_{e v, s}^{d^{\max }} \geq 0 \quad \forall e v, s \\
& 0 \leq P_{e v}^{\max }-P O P_{e v}-R_{e v}^{d o w n^{s}} \perp \mu_{e v, s}^{\text {down }} \geq 0 \quad \forall e v, s \\
& 0 \leq R_{g}^{u p^{\max }}-R_{g}^{u p^{s}} \perp \mu_{g, s}^{u p^{\max }} \geq 0 \quad \forall g, s \\
& 0 \leq R_{g}^{u p^{\max }}-P_{g}^{D A}-R_{g}^{u p^{s}} \perp \mu_{g, s}^{u p} \geq 0 \quad \forall g, s \\
& 0 \leq R_{g}^{d^{\max }}-R_{g}^{\text {down }}{ }^{s} \perp \mu_{g, s}^{d^{\max }} \geq 0 \quad \forall g, s \\
& 0 \leq P_{g}^{D A}-R_{g}^{\text {down }}{ }^{s} \perp \mu_{g, s}^{\text {down }} \geq 0 \quad \forall g, s \\
& 0 \leq P_{w}^{R T^{s}}-P_{w}^{C^{s}} \perp \mu_{w, t}^{c^{\max }} \geq 0 \quad \forall w, s \\
& 0 \leq L_{d}^{\max }-L_{d}^{C^{s}} \perp \mu_{d, s}^{c^{\max }} \geq 0 \quad \forall d, s \\
& 0 \leq R_{e v}^{u p^{s}} \perp \mu_{e v, s}^{u p^{m i n}} \geq 0 \quad \forall e v, s \\
& 0 \leq R_{e v}^{d o w n^{s}} \perp \mu_{e v, s}^{d^{m i n}} \geq 0 \quad \forall e v, s \\
& 0 \leq R_{g}^{u p^{s}} \perp \mu_{g, s}^{u p^{m i n}} \geq 0 \quad \forall g, s
\end{aligned}
$$




$$
\begin{array}{ll}
0 \leq R_{g}^{\text {down }}{ }^{s} \perp \mu_{g, s}^{d^{\text {min }}} \geq 0 & \forall g, s \\
0 \leq P_{w}^{C^{s}} \perp \mu_{w, s}^{c^{m i n}} \geq 0 & \forall w, s \\
0 \leq L_{d}^{C^{s}} \perp \mu_{d, s}^{c^{m i n}} \geq 0 & \forall d, s
\end{array}
$$

Nonlinear terms in the strong duality equality (5.7a) are replaced by the complementarity conditions as follows:

$$
\begin{array}{ll}
0 \leq E_{e v}^{D A}-E_{e v}^{M a x} \perp \mu_{e v}^{E E V^{\max }} \geq 0 & \forall e v, t \\
0 \leq E_{e v}^{M i n}-E_{e v}^{D A} \perp \mu_{e v}^{E E V^{\text {min }}} \geq 0 & \forall e v, t
\end{array}
$$

\subsubsection{Linearization of Complementarity Conditions}

Similar to Chapter 4, all complementarity conditions in the form of $0 \leq P \perp \mu \geq$ 0 can be linearized by

$$
P \geq 0, \mu \geq 0, \mu \leq b \cdot M_{1}, P \leq 1-b \cdot M_{2}
$$

where $b$ is an auxiliary binary variable, and $M_{1}$ and $M_{2}$ are large enough constants.

\subsubsection{Linearization of Nonlinear Terms Comprising $\emptyset_{j}^{E V}, \emptyset_{j, s}^{R T}$, and $\emptyset_{j}^{D A}$}

The nonlinear terms comprising $\emptyset_{j}^{E V}, \emptyset_{j, s}^{R T}$, and $\emptyset_{j}^{D A}$ are linearized by parameterizing the KKT conditions of the EPEC. Since the set of multipliers forms a ray and some degrees of freedom, nonlinear terms are linearized by parameterizing [88]. 


\subsubsection{Diagonalization Algorithm}

Every EPEC solution is not essentially a Nash equilibrium. Based on the method proposed in [71], a one-iteration diagonalization algorithm is implemented in this thesis as shown in Fig. 5.3 to test each solution to be a real Nash equilibrium based on [91].

For the results Q1 and Q2 achieved from the EPEC problem for firms Y1 and Y2, the results Q1 of firm Y1 is fixed, and then the MPEC problem related to its rival producer Y2 results in Q2*. If the results derived from the MPEC related to each firm are equal to the results obtained from the EPEC problem, the results Q1 and Q2 are a Nash equilibrium.

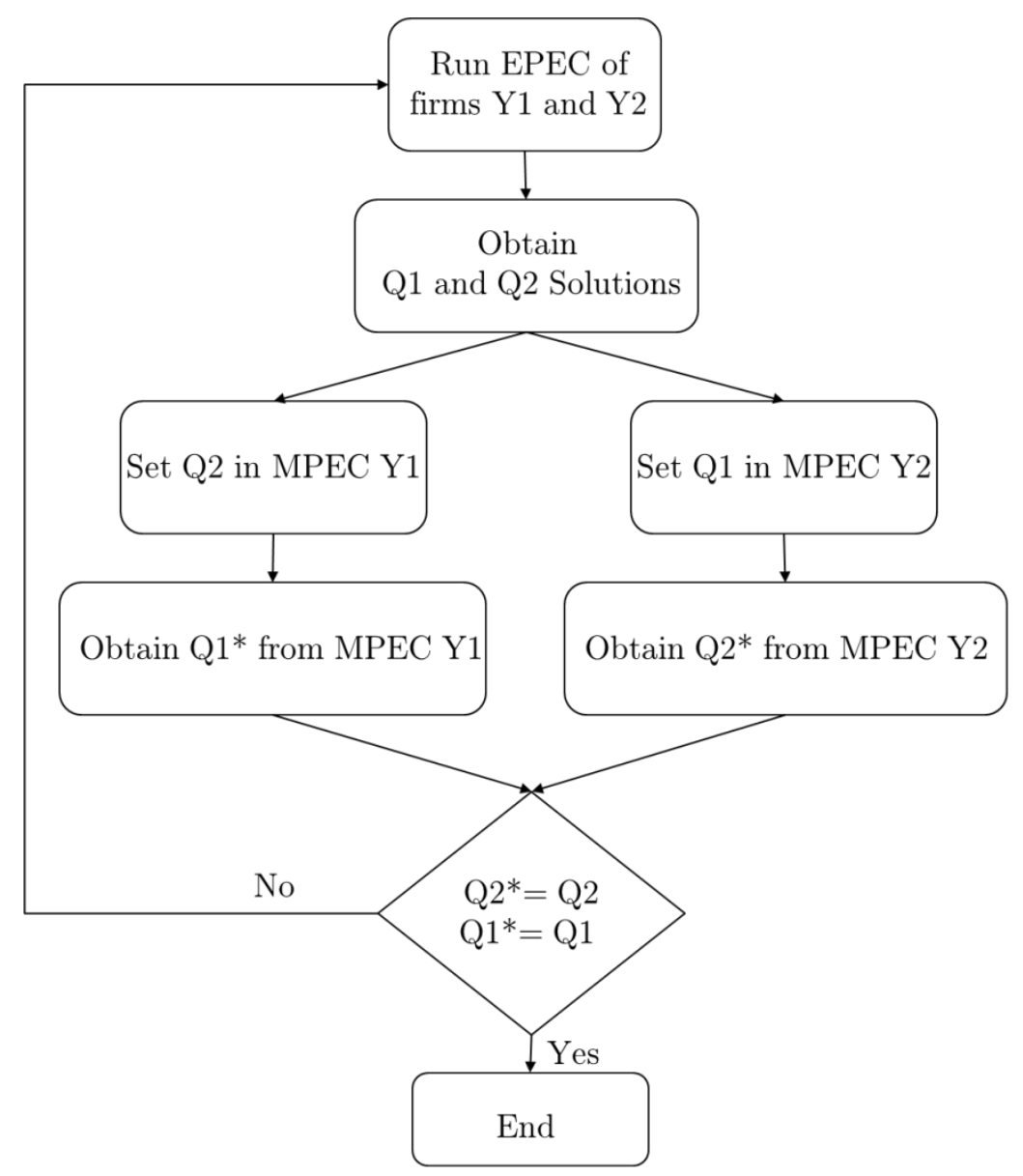

Fig. 5.3 A one-iteration diagonalization algorithm 


\subsection{Case Studies and Numerical Results}

To test the proposed model, the total demand is considered as $3.5 \mathrm{GWh}$ with two demand blocks of 2 , and $1.5 \mathrm{GWh}$ at the prices of $\$ 45 / \mathrm{MWh}$ and $\$ 35 / \mathrm{MWh}$. The system has two WGenCo and four CGenCos including nuclear, coal, oil and gas units which are assumed to be dispatchable. Generators' data are listed in Table 5.1. The total power capacities of wind and dispatchable units are the percentages of a total installed CGenCo power capacity ( $\mathrm{P}^{\wedge} \mathrm{CGenCo}$ ) of $3.5 \mathrm{GW}$ (see Table 5.1). The system has two EV aggregators with different EV numbers. The maximum EV charging power is assumed to be $7.3 \mathrm{~kW}$, and the energy capacity of each EV is $27.4 \mathrm{kWh}$. Average annual driving distance of an EV is assumed to be $20,000 \mathrm{~km}$ with an average daily distance of $52.91 \mathrm{~km}$. The required energy for an $\mathrm{EV}$ is $9 \mathrm{kWh} /$ day with an average of $5.87 \mathrm{~km} / \mathrm{kWh}[35]$.

Table 5.1

Summary of Data

\begin{tabular}{l|c|c|c|c}
\hline Unit Type & Nuclear & Coal & Oil & Gas \\
\hline$P_{g}^{\text {Max }}(\mathrm{MW})$ & 1500 & 1100 & 500 & 400 \\
\hline Cg $(\$ / \mathrm{MWh})$ & 12 & 20 & 25 & 30 \\
\hline$R_{g, \text { Max }}^{\text {UP }} R_{g, \text { Min }}^{\text {DOWN }}\left(\%\right.$ of $\left.P_{g}^{\text {Max }}\right)$ & 0 & $25 \%$ & $50 \%$ & $50 \%$ \\
\hline Total Demand $(\mathrm{GW})$ & \multicolumn{5}{|c}{3.5} \\
\hline$P^{\text {CGenco }}(\mathrm{GW})$ & 3.5 \\
\hline Total Wind $(\mathrm{GW})$ & \multicolumn{5}{|c}{$22 \%$ of $P^{\text {CGenCo }}$} \\
\hline
\end{tabular}

Three firms associated with different combination of units are considered in four case studies as described in Table 5.2. In addition, three different cases are 
considered for EV numbers including, without EV, 50,000 and 100,000 for each EV aggregator.

We analyse the market output in terms of the payoff, price, and generation and demand dispatch. Note that there are an infinite number of Nash equilibria, whereas no firm wants to change its offering strategy. Therefore, we cannot guarantee that there are a finite or an infinite number of solutions [88].

Table 5.2

The Combination of WGenCos, CGenCos, and EV Aggregators in Three Firms

\begin{tabular}{l|l|l|l}
\hline & Firm 1 & Firm 2 & Firm 3 \\
\hline Case 1 & $\begin{array}{l}\text { All WGenCos, CGenCos, } \\
\text { and EV aggregators }\end{array}$ & - \\
\hline Case 2 & $\begin{array}{l}\text { WGenCo1, Nuclear, and } \\
\text { Oil units, and EV } \\
\text { aggregator1 }\end{array}$ & $\begin{array}{l}\text { Gas units, and EV } \\
\text { aggregator2 }\end{array}$ & - \\
\hline Case 3 & $\begin{array}{l}\text { All WGenCos, and } \\
\text { CGenCos }\end{array}$ & All EV aggregators & - \\
\hline Case 4 & All WGenCos & All CGenCos & All EV aggregators \\
\hline
\end{tabular}

\subsubsection{Payoff and Price Analysis}

In all cases, some units offer the minimum bid price of the demands in the dayahead market. Therefore, the day-ahead market price and EV tariff are cleared in the price of $\$ 35 / \mathrm{MWh}$. The output of the strategic firms is consistent with the outcomes of [13] and [88]. Table 5.3 demonstrate the details of the numerical results of the payoffs in different cases. 
In Case 1 ( or single firm), all CGenCos, WGenCos and EV aggregators are considered as a single firm. The total profit of the firm increases with more EV numbers. However, the expected profit of EV aggregators is negative. The total expected profit of the single firm in Case 1 is higher than other cases as expected.

Table 5.3

Firms' Payoff in Different Cases

\begin{tabular}{|c|c|c|c|c|c|}
\hline & & Firm 1 & Firm 2 & Firm 3 & Total \\
\hline \multirow{3}{*}{ Case 1} & Without EV & $\$ 70550$ & - & - & $\$ 70550$ \\
\hline & $100,000 \mathrm{EV}$ & $\$ 72600$ & - & - & $\$ 72600$ \\
\hline & $200,000 \mathrm{EV}$ & $\$ 72855$ & - & - & $\$ 72855$ \\
\hline \multirow{3}{*}{ Case 2} & Without EV & $\$ 25740$ & $\$ 43550$ & - & $\$ 43550$ \\
\hline & $100,000 \mathrm{EV}$ & $\$ 27575$ & $\$ 44880$ & - & $\$ 72455$ \\
\hline & $200,000 \mathrm{EV}$ & $\$ 27660$ & $\$ 45030$ & - & $\$ 72690$ \\
\hline \multirow{3}{*}{ Case 3} & Without EV & $\$ 70550$ & - & - & $\$ 70550$ \\
\hline & $100,000 \mathrm{EV}$ & $\$ 72170$ & $\$ 0$ & - & $\$ 72170$ \\
\hline & $200,000 \mathrm{EV}$ & 72170 & $\$ 0$ & - & $\$ 72170$ \\
\hline \multirow{3}{*}{ Case 4} & Without EV & $\$ 14630$ & $\$ 50650$ & - & $\$ 65280$ \\
\hline & $100,000 \mathrm{EV}$ & $\$ 12880$ & $\$ 50650$ & $\$ 1260$ & $\$ 64790$ \\
\hline & $200,000 \mathrm{EV}$ & $\$ 12880$ & $\$ 52398$ & $\$ 2489.333$ & $\$ 67767.33$ \\
\hline
\end{tabular}


In Case 2, each two firms consist of a CGenCo, a WGenCo and an EV aggregator. The total profit of each firm increases with higher EV numbers and the expected profit of EV aggregators is positive. However, the expected profit of EV aggregators is lower than dispatchable units of CGenCos.

In Case 3, EV aggregators as an individual firm are the rival of the other firm including conventional dispatchable units and WGenCos. It is observed that wind power deviations are compensated by the dispatchable units included in that firm. Therefore, the expected profit of EV aggregators is zero. Also, the results of Cases 1 and 3 for without EV are the same as expected.

In Case 4 (or three separate firms), each CGenCo, WGenCo and EV aggregator is considered as an individual firm. The EV aggregators and dispatchable units of the CGenCo compete together to provide ancillary services for wind power deviation of the WGenCos. For 200,000 EVs, the expected profit of the EV aggregator is higher than other dispatchable units of CGenCos because of the dominant regulation ancillary capacity of the EV aggregators. However, the total expected profit of all firms in Case 4 is lower than in other cases.

In summary, the coordination strategy (Cases 1 and 2) in comparison with incoordination strategy (Cases 3 and 4) is more profitable and beneficial with increasing EV penetration.

Tables C.1-C.4 demonstrate the details of the numerical results of the payoff, and prices for the four cases in Appendix C.

\subsubsection{Demand and Generation Dispatch Analysis}

Table 5.4 shows real-time wind power deviations and regulation up/down of the units to compensate those deviations in different scenarios for 200,000 EVs. In Case 2, the WGenCo of each firm trades with the dispatchable units owned by 
that firm in the real-time market. For instance, wind power deviations in WGenCo 1 are compensated through the regulation up/down arranged by the Oil units and EV aggregator 1 into Firm 1. In Case 3, EV aggregators participate in the market as a separate firm (Firm 1) and compete with another firm including conventional dispatchable units and WGenCos. It is observed that wind power deviations are compensated by the dispatchable units included in that firm. Therefore, the EV aggregators do not contribute in regulation ancillary services. In Case 4, each CGenCo and EV aggregator compete together to allocate more share for ancillary services to compensate the real-time wind power deviations. In all cases, the regulation up is generally provided from the comparatively lowercost units, while the regulation down is generally procured through the comparatively expensive units due to the objective function solving the EPEC.

Tables 5.5 and 5.6 demonstrate the demand curtailment and wind power curtailment in four cases for 200,000 and 100,000 EVs, respectively. The wind power curtailments are zero in all cases, since the MO maximizes social welfare in the objective function. The demand curtailment (cut-off demand) of LSE in the real-time markets is zero except in Case 4 due to three competing individual firms including EV aggregator, WGenCo, and CGenCo together.

Tables 5.7 and 5.8 show regulation-up/down power of the EV aggregators and demand curtailment in Case 4 and Case 2, respectively. In both two cases, the EV aggregators contribute more regulation ancillary services with higher EV penetration. Moreover, the demand curtailment of LSE decreases with the increase in EV numbers.

Tables C.5-C.8 demonstrate the details of the numerical results of the demand, and generation dispatch for the four cases in Appendix C. 
Table 5.4

Real-time Wind Power Deviations and Regulation up/down of the Units for $200,000 \mathrm{EVs}$

\begin{tabular}{|c|c|c|c|c|c|c|c|c|c|c|c|c|}
\hline & \multicolumn{3}{|c|}{ Case 1} & \multicolumn{3}{|c|}{ Case 2} & \multicolumn{3}{|c|}{ Case 3} & \multicolumn{3}{|c|}{ Case 4} \\
\hline Scenarios & $\mathrm{S}_{1}$ & $\mathrm{~S}_{2}$ & $\mathrm{~S}_{3}$ & $\mathrm{~S}_{1}$ & $\mathrm{~S}_{2}$ & $\mathrm{~S}_{3}$ & $\mathrm{~S}_{1}$ & $\mathrm{~S}_{2}$ & $\mathrm{~S}_{3}$ & $\mathrm{~S}_{1}$ & $\mathrm{~S}_{2}$ & $\mathrm{~S}_{3}$ \\
\hline Wind Unit 1 & +200 & -20 & -150 & +200 & -20 & -150 & +200 & -20 & -150 & +200 & -20 & -150 \\
\hline Wind Unit 2 & +50 & -20 & -100 & +50 & -20 & -100 & +50 & -20 & -100 & +50 & -20 & -100 \\
\hline Nuclear Unit & 0 & 0 & 0 & 0 & 0 & 0 & 0 & 0 & 0 & 0 & 0 & 0 \\
\hline Coal Unit & 0 & 0 & 0 & 0 & +40 & +20 & 0 & 0 & 0 & 0 & 0 & 0 \\
\hline Oil Unit & -50 & 0 & 0 & -50 & +20 & +40 & -50 & 50 & 50 & -250 & -250 & +250 \\
\hline Gas Unit & -200 & 35 & 35 & -200 & -20 & 0 & -200 & -10 & 200 & -108 & +200 & -108 \\
\hline EV Agg. 1 & 0 & 5 & 105 & 0 & 0 & +110 & 0 & 0 & 0 & 0 & 80.48 & 0 \\
\hline EV Agg. 2 & 0 & 0 & 110 & 0 & 0 & +80 & 0 & 0 & 0 & 73.9 & 0 & 60.84 \\
\hline
\end{tabular}

Firm 1

Firm 2

Firm 3

Table 5.5

Demand Curtailment and Wind Power Curtailment for 200,000 EVs

\begin{tabular}{|c||c|c|c||c|c|c||c|c|c||c|c|c||}
\hline & \multicolumn{3}{|c||}{ Case 1 } & \multicolumn{3}{c||}{ Case 2 } & \multicolumn{3}{c||}{ Case 3 } & \multicolumn{3}{c||}{ Case 4 } \\
\hline Scenarios & $\mathrm{S}_{1}$ & $\mathrm{~S}_{2}$ & $\mathrm{~S}_{3}$ & $\mathrm{~S}_{1}$ & $\mathrm{~S}_{2}$ & $\mathrm{~S}_{3}$ & $\mathrm{~S}_{1}$ & $\mathrm{~S}_{2}$ & $\mathrm{~S}_{3}$ & $\mathrm{~S}_{1}$ & $\mathrm{~S}_{2}$ & $\mathrm{~S}_{3}$ \\
\hline $\begin{array}{c}\text { Demand } \\
\text { Curtailment }\end{array}$ & 0 & 0 & 0 & 0 & 0 & 0 & 0 & 0 & 0 & 34.1 & 9.52 & 47.16 \\
\hline $\begin{array}{c}\text { Wind Power } \\
\text { Curtailment }\end{array}$ & 0 & 0 & 0 & 0 & 0 & 0 & 0 & 0 & 0 & 0 & 0 & 0 \\
\hline
\end{tabular}


Table 5.6

Demand Curtailment and Wind Power Curtailment for 100,000 EVs

\begin{tabular}{|c||c|c|c|c|c|c||c|c|c||c|c|c||}
\hline \multicolumn{1}{|c||}{} & \multicolumn{3}{c||}{ Case 1 } & \multicolumn{3}{c||}{ Case 2 } & \multicolumn{3}{c||}{ Case 3} & \multicolumn{3}{c||}{ Case 4} \\
\hline Scenarios & $\mathrm{S}_{1}$ & $\mathrm{~S}_{2}$ & $\mathrm{~S}_{3}$ & $\mathrm{~S}_{1}$ & $\mathrm{~S}_{2}$ & $\mathrm{~S}_{3}$ & $\mathrm{~S}_{1}$ & $\mathrm{~S}_{2}$ & $\mathrm{~S}_{3}$ & $\mathrm{~S}_{1}$ & $\mathrm{~S}_{2}$ & $\mathrm{~S}_{3}$ \\
\hline $\begin{array}{c}\text { Demand } \\
\text { Curtailment }\end{array}$ & 0 & 0 & 0 & 0 & 0 & 0 & 0 & 0 & 0 & 163.8 & 36.36 & 181.8 \\
\hline $\begin{array}{c}\text { Wind Power } \\
\text { Curtailment }\end{array}$ & 0 & 0 & 0 & 0 & 0 & 0 & 0 & 0 & 0 & 0 & 0 & 0 \\
\hline
\end{tabular}

Table 5.7

Regulation up/down of the EV aggregators and Demand Curtailment in Case 4

\begin{tabular}{|c|c|c|c|c|c|c|}
\hline & \multicolumn{2}{|c|}{100,000 EVs } & \multicolumn{3}{c|}{200,000 EVs } \\
\hline Scenarios & $\mathrm{S}_{1}$ & $\mathrm{~S}_{2}$ & $\mathrm{~S}_{3}$ & $\mathrm{~S}_{1}$ & $\mathrm{~S}_{2}$ & $\mathrm{~S}_{3}$ \\
\hline Demand Curtailment of LSE & 163.8 & 36.36 & 181.8 & 34.1 & 9.52 & 47.16 \\
\hline Total Regulation up/down of EV Agg. & 36.2 & 53.64 & 18.2 & 73.9 & 80.48 & 60.84 \\
\hline
\end{tabular}

Table 5.8

Regulation up/down of the EV aggregators and Demand Curtailment in Case 2

\begin{tabular}{|c|c|c|c|c|c|c|}
\hline & \multicolumn{3}{|c|}{100,000 EVs } & \multicolumn{3}{c|}{200,000 EVs } \\
\hline Scenarios & $\mathrm{S}_{1}$ & $\mathrm{~S}_{2}$ & $\mathrm{~S}_{3}$ & $\mathrm{~S}_{1}$ & $\mathrm{~S}_{2}$ & $\mathrm{~S}_{3}$ \\
\hline Demand Curtailment of LSE & 0 & 0 & 0 & 0 & 0 & 0 \\
\hline Total Regulation up/down of EV Agg. & 0 & 0 & 110 & 0 & 0 & 190 \\
\hline
\end{tabular}




\subsection{Summary}

In this chapter, the optimal bidding/offering strategy for CGenCo, WGenCo, and EV load aggregator providing the energy and ancillary services is modelled for multiple firms at wholesale-level markets. At the EV-level, EV owners connect to the EV aggregator in order to take part in the market indirectly for obtaining maximum EV energy capacity and optimal charging tariff based on achieved dayahead and real-time market data.

Therefore, the EV aggregator as a new player competes with the conventional dispatchable units to provide the energy and ancillary services. An equilibrium approach is used to model the interactions of the strategic firms and is solved by EPEC.

Some case studies are used to validate the outcomes of the model. Moreover, impact of the increasing EV numbers in the model is investigated.

The numerical results show the effectiveness of the coordination strategy which is profitable and beneficial with increasing $\mathrm{EV}$ penetration in comparison with the incoordination strategy.

In other words, EV aggregators as an individual firm could not compete with other conventional dispatchable companies. Hence, merging EV aggregators in CGenCos' and WGenCos' portfolio would increase the payoff of EV aggregators and strategic firms. However, a sufficient EV number is a significant factor to affect market and EV aggregator outputs. 



\section{Chapter 6}

\section{Conclusion and Future Research}

In this final chapter, a summary of the thesis is first presented. Next, a list of relevant contributions of the thesis is provided. Finally, some suggestions for future research are recommended.

\subsection{Summary}

This thesis focused on the impact of the participation of the EV load aggregator, and the coordination strategy on the market outcomes and prices. The coordination strategy means coordination between the EV load aggregator and generating companies through V2G technology.

In this dissertation, we addressed the two matters below:

1) Development of a power exchange between the EV load aggregators and WGenCos considered as price-takers in the energy and ancillary service markets (Chapter 3). 
2) Development of a power exchange between the EV load aggregators and all generating companies considered as price-makers in the single and multiple firms (Chapters 4 and 5).

\subsubsection{Coordinating EV load aggregators and WGenCos as price-takers}

We developed a two-stage stochastic optimal offering/bidding strategy model for the coordinated EV-Wind units participating in the day-ahead energy, balancing, and regulation markets.

An objective function as a single optimization problem maximized the profit of coordinated WGenCo and EV aggregator associated with equality or inequality constraints.

We considered uncertainties in wind speed, energy prices, and EV owners' behavior based on driving patterns.

We investigated three different strategies including conventional systems (WGenCo without storage), WGenCo with an energy storage system, and a power system with a coordinated EV-Wind energy exchange.

The numerical results showed that the effective coordination between a WGenCo participating and an EV aggregator participating in the energy and ancillary service markets increases the WGenCo's competitiveness and mitigates wind and EV energy imbalance threats.

\subsubsection{Coordinating EV load aggregators and all GenCos as price-makers}

We developed a stochastic optimal bidding/offering strategy for the EV load aggregator providing the energy and ancillary services in coordination with single 
and multiple strategic firms in a pool-based electricity market with endogenous formation of day-ahead and real-time prices, and EV aggregator tariff. To consider EV aggregator tariff as an endogenous variable, we defined two levels in the electricity market comprising EV-level and wholesale-level markets. At wholesale-level market, MO runs day-ahead market and real-time market clearing. At EV-level market, the EV aggregator connect to EV owners to decide maximum EV energy capacity and optimal charging tariff based on achieved dayahead and real-time data.

We used a bilevel model to model the behaviour of proposed markets. A bilevel problem includes an upper-level problem and a set of lower-level problems which are limited by the upper and lower equality and inequality constraints. The upper-level problem represents the strategic firm's profit maximization. The lower-level problems include the day-ahead market, real-time market, and EV energy market clearing which aim to maximize the social welfare and are subject to the power balance, and power limits.

We studied single and multiple strategic firms in the proposed markets. For single firm, a bilevel model including multi-optimization problems converts into a single optimization problem as a single-level stochastic MPEC. For multiple firms, multiple stochastic MPECs constitute an EPEC. Finally, all single and multiple firms' problems are linearized by formulating and solving an MILP problem.

The numerical results showed the effectiveness of the coordination strategy, which is profitable and beneficial with increasing EV penetration in comparison with the incoordination strategy. We concluded that EV aggregators as an individual firm could not compete with other conventional, dispatchable companies. Hence, merging EV aggregators in CGenCos' and WGenCos' portfolio would increase the payoff of EV aggregators and strategic firms. However, a 
sufficient EV number is a significant factor to affect market and EV aggregator outputs.

Moreover, the numerical results showed that the EV tariff and numbers at EVlevel can influence the market price and power generation at wholesale-level in the electricity market. In addition, the high penetration of EVs leads to increasing the wind power penetration and reducing the wind power curtailment.

\subsection{Main Contributions}

The main contributions of the thesis work which have been declared in the introduction of each chapter are classified here in more solid expressions as follows:

1. For the coordinated EV-Wind and the ESS-Wind, developing a two-stage SLP-based optimal offering/bidding strategy model in the day-ahead energy, balancing, and regulation markets.

2. Comprehensive comparisons of three strategies including the coordinated EV-Wind, the ESS-Wind, and conventional systems (WGenCo without energy storage).

3. For the single and multiple firms including the coordinated EV aggregator, CGenCos and WGenCos, developing an optimal bidding/offering strategy model in a pool-based electricity market with endogenous formation of day-ahead and real-time prices, and EV aggregator tariff.

4. Defining EV-level and wholesale-level in the electricity market to consider impact of EV aggregator tariff on the price and market outputs.

5. Proposing formulation of a stochastic bilevel optimization problem including an upper-level problem and three lower-level problems for the 
sake of a) the strategic firm's profit maximization, and b) day-ahead and real-time social welfare maximization, and c) EV owner's battery energy maximization, respectively.

6. Comprehensive comparisons of different case studies including a) coordination and incoordination strategies; b) with and without EVs; c) the impact of different EV numbers; d) fixed-rate and ToU tariff; e) single and multiple firms.

7. Considering the uncertainties associated with wind forecast, energy price, and EV owners' behavior based on driving patterns.

\subsection{Recommendations for Future Research}

The concept of $\mathrm{V} 2 \mathrm{G}$ in the electricity market is indeed a new and interesting research area to design the future structure and architecture of the electricity market. This concluding section proposes some relevant areas for future research as follows:

1. Considering risk-constrained profit-maximization of a strategic firm such as conditional value at risk, $(\mathrm{CVaR})$ to investigate the impact of risk aversion on market decisions.

2. Considering the strategic LSEs through their demand function bids to award more flexibility for normal consumers as active players.

3. Integrating transmission networks and security constraints including a set of plausible contingencies, i.e., generators and transmission line outages into the market clearing problems. 
4. Investigating the impact of subsidizing renewable energies and EVs policies on the economics of CGenCos and the electricity market.

5. Studying the impact of the proposed market considered in this thesis on other markets such as futures market, and gas and oil markets.

6. Comparing a robust model with a stochastic programming model considered in the thesis work.

7. Developing an analytical sensitivity analysis tool to evaluate the effect of the diverse parameters on the market outputs. 


\section{Appendix A}

\section{Scenario Generation and Reduction}

\section{Techniques}

There are several different scenario generation and reduction techniques for stochastic programming [59]. The Monte Carlo simulations are applied to generate scenarios in [59]. In [60], the time series models are used to generate scenarios for prices in electricity markets. The most common scenario-reduction technique is based on Kantorovich distance [61]. In [62], a scenario generation for price forecasting is based on the roulette wheel mechanism. In this thesis, scenario generation and reduction techniques are used for simulating wind speed, energy price, and the number of EVs engaged as follows.

\section{A.1 Wind and Energy Price Scenarios}

Wind speed forecasting for the next day can be obtained from numerical meteorological programs, however, forecasts are never perfect. The ARMA model 
is used to simulate wind speed forecast errors [63-64,57]. The ARMA (p, q) model for a stochastic process $\mathrm{X}$ is defined as:

$\mathrm{x}(\mathrm{t})=\sum_{j=1}^{p} \alpha(j) \cdot x t-j+z t+\sum_{j=1}^{q} \beta(j) \cdot z(t-j)$

where $\mathrm{p}$ is autoregressive parameters $\alpha_{1}, \alpha_{2}, \ldots, \alpha_{p}$, and $\mathrm{q}$ is moving average parameters $\beta_{1}, \beta_{2}, \ldots, \beta_{p} ; \mathrm{Z} \mathrm{t}$ is a random Gaussian variable with standard deviation $\sigma[57]$.

The estimation and adjustment of ARMA models have been investigated in literature. In this thesis, the first order of the ARMA model, ARMA $(1,1)$, is used to simulate wind speed forecasting errors. This approach has been suggested in $[64],[57]:$

$\Delta \mathrm{V} t=\alpha \Delta \mathrm{V} t-1+\mathrm{Zt}+\beta \mathrm{Z} t-1$

where $\Delta \mathrm{V} t$ is the wind speed forecast error at the time (t) forecast; and $\alpha$, and $\beta$ are parameters.

The estimation of parameters and $\beta$ for a given wind speed forecast is done as suggested in [63]. ARMA parameters are obtained by minimizing the difference in the root mean square error between the simulated ARMA model and the wind speed measurement data [64], [101].

The real wind speed $\mathrm{V}(\mathrm{t})$ is calculated as the sum of the wind speed forecast $\mathrm{V}^{\mathrm{f}} \mathrm{t}$ and the wind speed forecast error: 
$\mathrm{V}(\mathrm{t})=\mathrm{V}^{\mathrm{f}}(t)+\Delta \mathrm{V} t$

Once a large number of scenarios are generated, the wind speed scenarios are transformed into power scenarios through the power conversion curve for each wind turbine [12], [65].

In addition, the process of scenario generation using the ARMA $(1,1)$ model is as follows [57].

Step 1: Initialize the scenario counter: $\mathrm{s} \leftarrow 0$.

Step 2: Update the scenario counter and initialize the time period counter: $\mathrm{s} \leftarrow \mathrm{s}+1, \mathrm{t} \leftarrow 0$

Step 3: Update the time period counter: $\mathrm{t} \leftarrow \mathrm{t}+1$.

Step 4: Randomly generate $\mathrm{Z}_{\mathrm{t}} \sim \mathrm{N}(0, \sigma)$.

Step 5: Evaluate $\Delta \mathrm{Vt}^{s}$.

Step 6: If $t<N T$ go to Step 3), otherwise go to Step 7)

Step 7: If $\omega<N$ go to Step 2), otherwise the scenario-generation process concludes.

Similarly, ARIMA models have been applied to forecast electricity prices, which appear non-stationary when the processes present a periodic or seasonal pattern [57], [102].

\section{A.2 EV Number Scenarios}

Any driving profile has a commute time including morning and evening with the start and end times, and a commute distance. Major commuting would normally begin between $7 \mathrm{AM}$ and $9 \mathrm{AM}$ to go to work and between $5 \mathrm{PM}$ and $8 \mathrm{PM}$ to come 
back from work. For all other times, the EVs are assumed to be available to be plugged into the electricity grid [66].

The EV availability at each interval has associated unplanned departure and arrival probabilities. The number of EVs is considered to be random, and Monte Carlo simulations are used to generate possible scenarios.

The total number of EVs is 1p.u. It is assumed that on average, from 2 AM to $5 \mathrm{AM}, 98 \%$ of EVs are plugged-in with a standard deviation of $5 \%$. For commute periods, on average, $20 \%$ of EVs are plugged-in with a standard deviation of $10 \%$, and during other periods it is assumed that $85 \%$ of EVs are plugged-in with a standard deviation of $20 \%$ [9]. The availability of EVs in various time periods is shown in Table A.1.

Table A.1

The Availability of EVs in Various Time Intervals

\begin{tabular}{|c|c|c|c|c|c|c|}
\hline Hour & $\begin{array}{l}2: 00 \\
5: 00\end{array}$ & $\begin{array}{l}\text { 5:00 } \\
\text { 7:00 }\end{array}$ & $\begin{array}{l}\text { 7:00 } \\
9: 00\end{array}$ & $\begin{array}{c}9: 00 \\
17: 00\end{array}$ & $\begin{array}{l}17: 00 \\
20: 00\end{array}$ & $\begin{array}{c}20: 00 \\
2: 00\end{array}$ \\
\hline St.dev (\%) & 5 & 20 & 10 & 20 & 10 & 20 \\
\hline Mean (pu) & 0.98 & 0.85 & 0.20 & 0.85 & 0.20 & 0.85 \\
\hline
\end{tabular}

\section{A.3 Scenario Reduction}

In stochastic optimization problems with various inherent uncertainties, a large number of scenarios can emerge. It can, therefore, be computationally expensive. Therefore, a technique for reducing the number of scenarios is required.

In this paper, the scenario reduction algorithm is based on [67], [68]. The basic idea of the scenario reduction is to eliminate scenarios with low-probabilities, and cluster similar scenarios [69], [101]. The new probability of a preserved scenario is 
determined as the sum of its initial probability and the probabilities of similar scenarios that have been eliminated. We used SCENRED as a tool for scenario reduction [69]. SCENRED contains three reduction algorithms: the fast backward method, a mix of fast backward/forward methods and a mix of fast backward/backward methods. The algorithms have different computational performances, and the choice of algorithms for a certain problem depends on the size of the problem and the required solution accuracy. The strategy used in [6769] recommends that the optimal deletion of scenarios should be determined by a conceptual algorithm called backward reduction. If the number of preserved scenarios is small (strong reduction), the optimal selection of a single scenario may be repeated recursively until a prescribed number of preserved scenarios is selected. This strategy provides the basic concept of the conceptual algorithm called forward selection. In this paper, the fast backward/forward method is selected to reduce the number of scenarios [68].

The uncertainties characterizing the stochastic data are modeled through a symmetric scenario tree [65]. Each branch of the scenario tree includes three nodes: the day-ahead energy price, wind power outputs, and EV penetration. The scenario tree generation process is described as follows:

Step 1: Generate a set of 10,000 day-ahead price scenarios $\left(A_{s}^{\rho}\right)$.

Step 2: Generate a set of 10,000 wind power scenarios $\left(A_{s}^{w}\right)$ for each of the day-ahead price scenarios.

Step 3: Generate a set of $10,000 \mathrm{EV}$ number scenarios $\left(A_{s}^{\text {ev }}\right)$ for each of the wind power scenarios. 


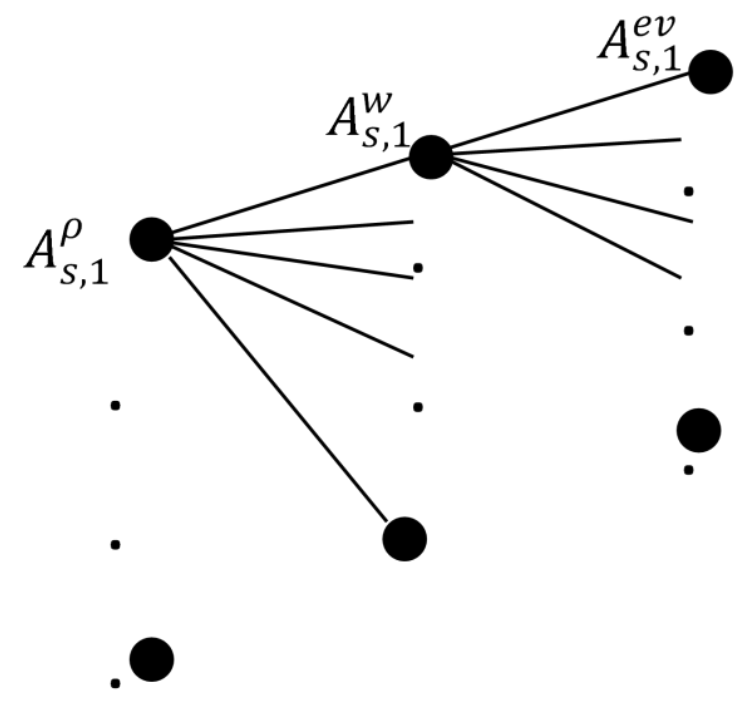

Fig. A.1 A path through the tree is called a scenario and consists of realizations of all random variables.

The total number of scenarios making the tree is $A_{s}=A_{s}^{\rho} \times A_{s}^{w} \times A_{s}^{e v}=10^{12}$, which makes the size of the tree too large for the optimization problem to be tractable. Therefore, a reduction technique proposed in [69] is used to reduce the number of $A_{s}^{\rho}, A_{s}^{w}$, and $A_{s}^{e v}$ to 10 each, i.e., the reduced tree consists of $10^{3}$ scenarios [65]. 


\section{Appendix B}

\section{Absolute Value of the Variable in the MILP}

To represent the absolute value of variable $x$ in a linear form for MILP formulation, the following equations are used:

$$
\begin{aligned}
& 0 \leq|x|-x \leq M a_{s, t, i}^{\Delta} \\
& 0 \leq|x|+x \leq M\left[1-a_{s, t, i}^{\Delta}\right] \\
& a_{s, t, i}^{\Delta} \in\{0,1\}, M \text { is a large positive number }
\end{aligned}
$$

For $>0$ :

$$
\text { if } a_{s, t, i}^{\Delta}=1 \rightarrow\left\{\begin{array}{c}
0 \leq|x|-x \leq M \\
|x|=-x
\end{array}\right. \text {,impossible }
$$




$$
\text { if } a_{s, t, i}^{\Delta}=0 \rightarrow\left\{\begin{array}{c}
|x|=x \\
0 \leq|x|+x \leq M
\end{array}\right. \text {, possible }
$$

For $x<0$ :

$$
\begin{aligned}
& \text { if } a_{s, t, i}^{\Delta}=1 \rightarrow\left\{\begin{array}{c}
0 \leq|x|-x \leq M \\
|x|=-x
\end{array},\right. \text { possible } \\
& \text { if } a_{s, t, i}^{\Delta}=0 \rightarrow\left\{\begin{array}{c}
|x|=x \\
0 \leq|x|+x \leq M
\end{array},\right. \text { impossible }
\end{aligned}
$$




\section{Appendix C}

\section{Additional Data}

This section includes the additional data on the multiple firms in coordination with EV load aggregators (chapter 5) to discuss the payoff and price, and demand and generation dispatch analysis for case studies 1-4 (Section 5.4). In Case 1 (or single firm), all CGenCos, WGenCos and EV aggregators are considered as a single firm. In Case 2, each two firms consist of a CGenCo, a WGenCo and an EV aggregator. In Case 3, EV aggregators as an individual firm are the rival of the other firm including conventional dispatchable units and WGenCos. In Case 4 (or three separate firms), each CGenCo, WGenCo and EV aggregator is considered as an individual firm.

Tables C.1-C.4 demonstrate the details of the numerical results of the payoff, and prices for the four cases.

Tables C.5-C.8 demonstrate the details of the numerical results of the demand, and generation dispatch for the four cases. 
Table C.1

The Results of Profit and Prices for Case 1

\begin{tabular}{|c|c|c|c|c|c|c|c|c|c|c|}
\hline \multirow{4}{*}{ 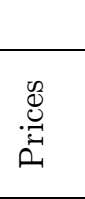 } & & \multicolumn{3}{|c|}{ Without EV } & \multicolumn{3}{|c|}{$100,000 \mathrm{EVs}$} & \multicolumn{3}{|c|}{$200,000 \mathrm{EVs}$} \\
\hline & DA Market Price $(\$ / M W h)$ & \multicolumn{3}{|c|}{35} & \multicolumn{3}{|c|}{35} & \multicolumn{3}{|c|}{35} \\
\hline & EV Tariff $(\$ / M W h)$ & \multicolumn{3}{|c|}{-} & \multicolumn{3}{|c|}{35} & \multicolumn{3}{|c|}{35} \\
\hline & RT Market Prices $(\$ /$ MWh $)$ & 70 & 70 & 0 & 0 & 0 & 70 & 70 & 0 & 0 \\
\hline \multirow{4}{*}{$\begin{array}{l}\text { 足 } \\
\stackrel{0}{0} \\
\text { D. }\end{array}$} & Total Profit $(\$)$ & \multicolumn{3}{|c|}{70550} & \multicolumn{3}{|c|}{72600} & \multicolumn{3}{|c|}{72855} \\
\hline & Total Profit of Firm $1(\$)$ & \multicolumn{3}{|c|}{70550} & \multicolumn{3}{|c|}{72600} & \multicolumn{3}{|c|}{72855} \\
\hline & Total Profit of Firm $2(\$)$ & \multicolumn{3}{|c|}{-} & \multicolumn{3}{|c|}{-} & \multicolumn{3}{|c|}{-} \\
\hline & Total Profit of Firm $3(\$)$ & \multicolumn{3}{|c|}{-} & \multicolumn{3}{|c|}{-} & \multicolumn{3}{|c|}{-} \\
\hline \multirow{3}{*}{ 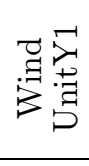 } & Expected Profit $(\$)$ & \multicolumn{3}{|c|}{12740} & \multicolumn{3}{|c|}{6300} & \multicolumn{3}{|c|}{13300} \\
\hline & DA Offer Price $(\$ / \mathrm{MWh})$ & \multicolumn{3}{|c|}{35} & & 35 & & & 35 & \\
\hline & RT Offer Prices (\$/MWh) & 0 & 0 & 0 & 0 & 0 & 0 & 70 & 0 & 0 \\
\hline$\nabla 5$ & Expected Profit $(\$)$ & & 714 & & & 420 & & & 770 & \\
\hline 蚂 & DA Offer Price $(\$ / M W h)$ & & 35 & & & 35 & & & 35 & \\
\hline 15 & RT Offer Prices $(\$ / M W h)$ & 0 & 0 & 0 & 0 & 0 & 0 & 70 & 0 & 0 \\
\hline$\tilde{\sigma} \bar{\tau}$ & Expected Profit $(\$)$ & & 3450 & & & 3450 & & & 450 & \\
\hline 兑莺 & DA Offer Price $(\$ / M W h)$ & & 35 & & & 0 & & & 0 & \\
\hline 公 & RT Offer Prices (\$/MWh) & 0 & 0 & 0 & 0 & 0 & 0 & 70 & 0 & 0 \\
\hline$\Rightarrow \rightleftharpoons$ & Expected Profit $(\$)$ & & 1650 & & & 1118 & & & 650 & \\
\hline ঠீ: & DA Offer Price $(\$ / M W h)$ & & 35 & & & 35 & & & 35 & \\
\hline & RT Offer Prices $(\$ / M W h)$ & 70 & 70 & 0 & 0 & 0 & 70 & 70 & 0 & 0 \\
\hline & Expected Profit $(\$)$ & & 2520 & & & 325 & & & 1550 & \\
\hline 窎 & DA Offer Price $(\$ / M W h)$ & & 35 & & & 35 & & & 35 & \\
\hline & RT Offer Prices (\$/MWh) & 70 & 70 & 0 & 0 & 0 & 70 & 70 & 0 & 0 \\
\hline & Expected Profit $(\$)$ & & -285 & & & 414 & & & 615 & \\
\hline 造莺 & DA Offer Price $(\$ / M W h)$ & & 35 & & & 35 & & & 35 & \\
\hline & RT Offer Prices $(\$ / M W h)$ & 70 & 70 & 0 & 0 & 0 & 70 & 70 & 0 & 0 \\
\hline $\begin{array}{l}\infty 00 \\
\infty\end{array}$ & Expected Profit $(\$)$ & & - & & & 770 & & & 154 & \\
\hline 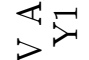 & DA Bid Price (\$/MWh) & & - & & & 35 & & & 35 & \\
\hline 亩 & RT Offer Prices $(\$ / M W h)$ & - & - & - & 0 & 0 & 70 & 70 & 0 & 0 \\
\hline $\begin{array}{l}60 \\
60\end{array}$ & Expected Profit $(\$)$ & & - & & & 321 & & & 154 & \\
\hline 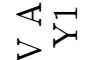 & DA Bid Price $(\$ / M W h)$ & & - & & & 35 & & & 35 & \\
\hline 宇 & RT Offer Prices $(\$ / M W h)$ & - & - & - & 0 & 0 & 70 & 70 & 0 & 0 \\
\hline
\end{tabular}


Table C.2

The Results of Profit and Prices for Case 2

\begin{tabular}{|c|c|c|c|c|c|c|c|c|c|c|}
\hline & & \multicolumn{3}{|c|}{ Without EV } & \multicolumn{3}{|c|}{$100,000 \mathrm{EVs}$} & \multicolumn{3}{|c|}{$200,000 \mathrm{EVs}$} \\
\hline \multirow{3}{*}{ 总 } & DA Market Price (\$/MWh) & \multicolumn{3}{|c|}{35} & \multicolumn{3}{|c|}{35} & \multicolumn{3}{|c|}{35} \\
\hline & EV Tariff $(\$ / M W h)$ & \multicolumn{3}{|c|}{-} & \multicolumn{3}{|c|}{35} & \multicolumn{3}{|c|}{35} \\
\hline & RT Market Prices $(\$ / \mathrm{MWh})$ & 70 & 22.5 & 27.5 & 25 & 27.5 & 46.25 & 20 & 30 & 47.5 \\
\hline \multirow{4}{*}{ 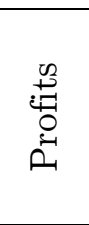 } & Total Profit $(\$)$ & \multicolumn{3}{|c|}{69290} & \multicolumn{3}{|c|}{72455} & \multicolumn{3}{|c|}{72690} \\
\hline & Total Profit of Firm $1(\$)$ & \multicolumn{3}{|c|}{25740} & \multicolumn{3}{|c|}{27575} & \multicolumn{3}{|c|}{27660} \\
\hline & Total Profit of Firm $2(\$)$ & \multicolumn{3}{|c|}{43550} & \multicolumn{3}{|c|}{44880} & \multicolumn{3}{|c|}{45030} \\
\hline & Total Profit of Firm $3(\$)$ & \multicolumn{3}{|c|}{-} & \multicolumn{3}{|c|}{-} & \multicolumn{3}{|c|}{-} \\
\hline \multirow{3}{*}{ 矛哥 } & Expected Profit $(\$)$ & \multicolumn{3}{|c|}{11170} & \multicolumn{3}{|c|}{8505} & \multicolumn{3}{|c|}{8210} \\
\hline & DA Offer Price (\$/MWh) & \multicolumn{3}{|c|}{35} & & 35 & & & 35 & \\
\hline & RT Offer Prices $(\$ / M W h)$ & 0 & 0 & 0 & 0 & 0 & 0 & 0 & 0 & 0 \\
\hline & Expected Profit (\$) & & 6220 & & & 518 & & & 5060 & \\
\hline 蚂 & DA Offer Price (\$/MWh) & & 35 & & & 35 & & & 35 & \\
\hline & RT Offer Prices $(\$ / M W h)$ & 0 & 0 & 0 & 0 & 0 & 0 & 0 & 0 & 0 \\
\hline & Expected Profit $(\$)$ & & 34500 & & & 3450 & & & 3450 & \\
\hline 总密 & DA Offer Price (\$/MWh) & & 0 & & & 0 & & & 0 & \\
\hline & RT Offer Prices $(\$ / M W h)$ & 0 & 0 & 0 & 0 & 0 & 0 & 0 & 0 & 0 \\
\hline & Expected Profit $(\$)$ & & 13420 & & & 1650 & & & 1650 & \\
\hline 离育 & DA Offer Price (\$/MWh) & & 35 & & & 35 & & & 35 & \\
\hline & RT Offer Prices $(\$ / M W h)$ & 70 & 22.7 & 27.5 & 25 & 27.5 & 46.25 & 20 & 30 & 47.5 \\
\hline $\mathfrak{\Im}$ & Expected Profit $(\$)$ & & 2830 & & & 4952 & & & 5070 & \\
\hline 窎 & DA Offer Price $(\$ / M W h)$ & & 35 & & & 35 & & & 35 & \\
\hline 5 & RT Offer Prices (\$/MWh) & 70 & 22.5 & 27.7 & 25 & 28 & 46.25 & 20 & 30 & 47.5 \\
\hline & Expected Profit $(\$)$ & & 1150 & & & & & & 2400 & \\
\hline 预: & DA Offer Price (\$/MWh) & & 35 & & & 35 & & & 34.9 & \\
\hline & RT Offer Prices (\$/MWh) & 70 & 22.7 & 27.5 & 25 & 27.5 & 46.25 & 20 & 30 & 47.5 \\
\hline$\infty_{\infty}^{\infty}$ & Expected Profit $(\$)$ & & - & & & 247. & & & 550 & \\
\hline$\underset{>>}{\infty}$ & DA Bid Price $(\$ / M W h)$ & & - & & & 35 & & & 35 & \\
\hline 空 & RT Offer Prices $(\$ / M W h)$ & - & - & - & 25 & 27.5 & 46.25 & 20 & 30 & 47.5 \\
\hline $\begin{array}{l}\infty \\
\infty \infty 0\end{array}$ & Expected Profit $(\$)$ & & - & & & 247. & & & 400 & \\
\hline$<i$ & DA Bid Price $(\$ / M W h)$ & & - & & & 35 & & & 35 & \\
\hline 宝 & RT Offer Prices $(\$ / M W h)$ & - & - & - & 25 & 27.5 & 46.25 & 20 & 30 & 47.5 \\
\hline
\end{tabular}


Table C.3

The Results of Profit and Prices for Case 3

\begin{tabular}{|c|c|c|c|c|c|c|c|c|c|c|}
\hline & & \multicolumn{3}{|c|}{ Without EV } & \multicolumn{3}{|c|}{$100,000 \mathrm{EVs}$} & \multicolumn{3}{|c|}{$200,000 \mathrm{EVs}$} \\
\hline \multirow{3}{*}{ 总 } & DA Market Price $(\$ / M W h)$ & \multicolumn{3}{|c|}{35} & \multicolumn{3}{|c|}{35} & \multicolumn{3}{|c|}{35} \\
\hline & EV Tariff $(\$ / M W h)$ & \multicolumn{3}{|c|}{-} & \multicolumn{3}{|c|}{35} & \multicolumn{3}{|c|}{35} \\
\hline & RT Market Prices $(\$ / \mathrm{MWh})$ & 70 & 70 & 0 & 0 & 70 & 70 & 0 & 70 & 0 \\
\hline \multirow{4}{*}{ 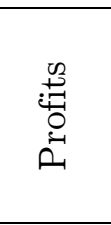 } & Total Profit $(\$)$ & \multicolumn{3}{|c|}{70550} & \multicolumn{3}{|c|}{72170} & \multicolumn{3}{|c|}{72170} \\
\hline & Total Profit of Firm $1(\$)$ & \multicolumn{3}{|c|}{70550} & \multicolumn{3}{|c|}{72170} & \multicolumn{3}{|c|}{72170} \\
\hline & Total Profit of Firm $2(\$)$ & \multicolumn{3}{|c|}{-} & \multicolumn{3}{|c|}{0} & \multicolumn{3}{|c|}{0} \\
\hline & Total Profit of Firm $3(\$)$ & \multicolumn{3}{|c|}{-} & \multicolumn{3}{|c|}{-} & \multicolumn{3}{|c|}{-} \\
\hline \multirow{3}{*}{ 营营 } & Expected Profit (\$) & \multicolumn{3}{|c|}{12740} & \multicolumn{3}{|c|}{5740} & \multicolumn{3}{|c|}{9940} \\
\hline & DA Offer Price (\$/MWh) & \multicolumn{3}{|c|}{35} & & 3 & & & 35 & \\
\hline & RT Offer Prices $(\$ / M W h)$ & 0 & 0 & 0 & 0 & 0 & 0 & 0 & 0 & 0 \\
\hline & Expected Profit $(\$)$ & & 714 & & & 36 & & & 644 & \\
\hline 青菏牙 & DA Offer Price (\$/MWh) & & 35 & & & 3 & & & 35 & \\
\hline & RT Offer Prices (\$/MWh) & 0 & 0 & 0 & 0 & 0 & 0 & 0 & 0 & 0 \\
\hline & Expected Profit (\$) & & 3450 & & & 345 & & & 3450 & \\
\hline 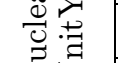 & DA Offer Price $(\$ / M W h)$ & & 0 & & & 0 & & & 0 & \\
\hline & RT Offer Prices (\$/MWh) & 0 & 0 & 0 & 0 & 0 & 0 & 0 & 0 & 0 \\
\hline & Expected Profit $(\$)$ & & 1650 & & & 175 & & & 1650 & \\
\hline 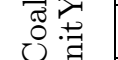 & DA Offer Price (\$/MWh) & & 35 & & & 3 & & & 35 & \\
\hline & RT Offer Prices (\$/MWh) & 70 & 70 & 0 & 0 & 70 & 70 & 0 & 70 & 0 \\
\hline$\Xi$ & Expected Profit $(\$)$ & & 2520 & & & 55 & & & 515 & \\
\hline 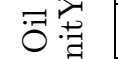 & DA Offer Price (\$/MWh) & & 35 & & & 3 & & & 35 & \\
\hline 5 & RT Offer Prices (\$/MWh) & 70 & 70 & 0 & 0 & 70 & 70 & 0 & 70 & 0 \\
\hline & Expected Profit (\$) & & -285 & & & 52 & & & -360 & \\
\hline 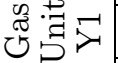 & DA Offer Price (\$/MWh) & & 35 & & & 3 & & & 35 & \\
\hline & RT Offer Prices (\$/MWh) & 70 & 70 & 0 & 0 & 70 & 70 & 0 & 70 & 0 \\
\hline$b_{\infty 0}^{\infty}$ & Expected Profit $(\$)$ & & - & & & 0 & & & 0 & \\
\hline$\sum_{i}^{\infty}$ & DA Bid Price $(\$ / M W h)$ & & - & & & 3 & & & 35 & \\
\hline 亩 & RT Offer Prices (\$/MWh) & - & - & - & 0 & 70 & 70 & 0 & 70 & 0 \\
\hline $\begin{array}{l}50 \\
80\end{array}$ & Expected Profit $(\$)$ & & - & & & 0 & & & 0 & \\
\hline$\underset{>}{i}$ & DA Bid Price (\$/MWh) & & - & & & 3 & & & 35 & \\
\hline & RT Offer Prices $(\$ / M W h)$ & - & - & - & 0 & 70 & 70 & 0 & 70 & 0 \\
\hline
\end{tabular}


Table C.4

The Results of Profit and Prices for Case 4

\begin{tabular}{|c|c|c|c|c|c|c|c|c|c|c|}
\hline & & \multicolumn{3}{|c|}{ Without EV } & \multicolumn{3}{|c|}{$100,000 \mathrm{EVs}$} & \multicolumn{3}{|c|}{$200,000 \mathrm{EVs}$} \\
\hline \multirow{3}{*}{$\begin{array}{l}\mathscr{U} \\
\stackrel{\mathscr{Z}}{\vec{D}} \\
D_{1}\end{array}$} & DA Market Price $(\$ / M W h)$ & \multicolumn{3}{|c|}{35} & \multicolumn{3}{|c|}{35} & \multicolumn{3}{|c|}{35} \\
\hline & EV Tariff $(\$ / M W h)$ & \multicolumn{3}{|c|}{-} & \multicolumn{3}{|c|}{35} & \multicolumn{3}{|c|}{35} \\
\hline & RT Market Prices $(\$ / M W h)$ & 70 & 70 & 70 & 70 & 70 & 70 & 70 & 70 & 70 \\
\hline \multirow{4}{*}{ 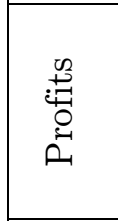 } & Total Profit $(\$)$ & \multicolumn{3}{|c|}{65280} & \multicolumn{3}{|c|}{64790} & \multicolumn{3}{|c|}{67767.333} \\
\hline & Total Profit of Firm $1(\$)$ & \multicolumn{3}{|c|}{14630} & \multicolumn{3}{|c|}{12880} & \multicolumn{3}{|c|}{12880} \\
\hline & Total Profit of Firm $2(\$)$ & \multicolumn{3}{|c|}{50650} & \multicolumn{3}{|c|}{50650} & \multicolumn{3}{|c|}{52398} \\
\hline & Total Profit of Firm $3(\$)$ & \multicolumn{3}{|c|}{-} & \multicolumn{3}{|c|}{1260} & \multicolumn{3}{|c|}{2489.333} \\
\hline \multirow{3}{*}{ 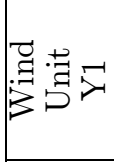 } & Expected Profit $(\$)$ & \multicolumn{3}{|c|}{8540} & \multicolumn{3}{|c|}{8540} & \multicolumn{3}{|c|}{8540} \\
\hline & DA Offer Price $(\$ / M W h)$ & \multicolumn{3}{|c|}{35} & & 3 & & & 35 & \\
\hline & RT Offer Prices $(\$ / M W h)$ & 0 & 0 & 0 & 0 & 0 & 0 & 0 & 0 & 0 \\
\hline & Expected Profit $(\$)$ & & 609 & & & 43 & & & 4340 & \\
\hline & DA Offer Price $(\$ / M W h)$ & & 35 & & & 0 & & & 35 & \\
\hline & RT Offer Prices (\$/MWh) & 0 & 0 & 0 & 0 & 0 & 0 & 0 & 0 & 0 \\
\hline & Expected Profit $(\$)$ & & 3450 & & & 345 & & & 34500 & \\
\hline 总富 & DA Offer Price $(\$ / M W h)$ & & 0 & & & 0 & & & 0 & \\
\hline & RT Offer Prices $(\$ / M W h)$ & 0 & 0 & 0 & 0 & 0 & 0 & 0 & 0 & 0 \\
\hline & Expected Profit $(\$)$ & & 1650 & & & 165 & & & 16500 & \\
\hline 号: & DA Offer Price $(\$ / M W h)$ & & 35 & & & 3 & & & 35 & \\
\hline & RT Offer Prices $(\$ / M W h)$ & 70 & 70 & 70 & 70 & 70 & 70 & 70 & 70 & 70 \\
\hline & Expected Profit $(\$)$ & & 250 & & & 25 & & & 250 & \\
\hline 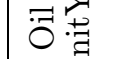 & DA Offer Price (\$/MWh) & & 35 & & & 3 & & & 35 & \\
\hline & RT Offer Prices $(\$ / M W h)$ & 70 & 70 & 70 & 70 & 70 & 70 & 78.57 & 78.57 & 70 \\
\hline & Expected Profit $(\$)$ & & -60 & & & -6 & & & 1148 & \\
\hline 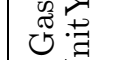 & DA Offer Price $(\$ / M W h)$ & & 35 & & & 3 & & & 34.96 & \\
\hline & RT Offer Prices (\$/MWh) & 70 & 70 & 70 & 70 & 70 & 70 & 73.81 & 70 & 77.61 \\
\hline $\begin{array}{l}50 \\
\infty \infty\end{array}$ & Expected Profit $(\$)$ & & - & & & 49 & & & 126.66 & \\
\hline$\sum_{>}^{\infty}$ & DA Bid Price $(\$ / M W h)$ & & - & & & 3 & & & 35 & \\
\hline & RT Offer Prices (\$/MWh) & - & - & - & 70 & 70 & 70 & 70 & 70 & 70 \\
\hline $\begin{array}{l}\infty \\
\infty\end{array}$ & Expected Profit $(\$)$ & & - & & & 77 & & & 362.66 & \\
\hline$<0$ & DA Bid Price $(\$ / M W h)$ & & - & & & 3 & & & 35 & \\
\hline & RT Offer Prices $(\$ / M W h)$ & - & - & - & 70 & 70 & 70 & 70 & 70 & 70 \\
\hline
\end{tabular}


Table C.5

The Results of Demand and Generation Dispatch for Case 1

\begin{tabular}{|c|c|c|c|c|c|c|c|c|c|c|}
\hline \multirow{2}{*}{\multicolumn{2}{|c|}{\begin{tabular}{|c|} 
(MW) \\
Demand Curtailment of LSE
\end{tabular}}} & \multicolumn{3}{|c|}{ Without EV } & \multicolumn{3}{|c|}{$100,000 \mathrm{EVs}$} & \multicolumn{3}{|c|}{$200,000 \mathrm{EVs}$} \\
\hline & & 0 & 0 & 0 & 0 & 0 & 0 & 0 & 0 & 0 \\
\hline & Total Wind Power Deviation & +250 & -40 & -250 & +250 & -40 & -250 & +250 & -40 & -250 \\
\hline \multirow{3}{*}{ 莬 } & DA Power & \multicolumn{3}{|c|}{300} & \multicolumn{3}{|c|}{300} & \multicolumn{3}{|c|}{300} \\
\hline & Wind Power Deviation & +200 & -20 & -150 & +200 & -20 & -150 & +200 & -20 & -150 \\
\hline & Wind Power Curtailment & 0 & 0 & 0 & 0 & 0 & 0 & 0 & 0 & 0 \\
\hline \multirow{3}{*}{ 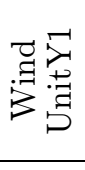 } & DA Power & \multicolumn{3}{|c|}{200} & \multicolumn{3}{|c|}{200} & \multicolumn{3}{|c|}{200} \\
\hline & Wind Power Deviation & +50 & -20 & -100 & +50 & -20 & -100 & +50 & -20 & -100 \\
\hline & Wind Power Curtailment & 0 & 0 & 0 & 0 & 0 & 0 & 0 & 0 & 0 \\
\hline \multirow{3}{*}{ 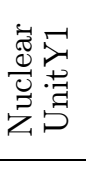 } & DA Power & \multicolumn{3}{|c|}{1500} & \multicolumn{3}{|c|}{1500} & \multicolumn{3}{|c|}{1500} \\
\hline & Regulation Up/Down & 0 & 0 & 0 & 0 & 0 & 0 & 0 & 0 & 0 \\
\hline & RT Power Output & 1500 & 1500 & 1500 & 1500 & 1500 & 1500 & 1500 & 1500 & 1500 \\
\hline \multirow{3}{*}{ 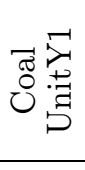 } & DA Power & \multicolumn{3}{|c|}{1100} & \multicolumn{3}{|c|}{1045.5} & \multicolumn{3}{|c|}{1100} \\
\hline & Regulation Up/Down & 0 & 0 & 0 & 54.5 & 54.5 & 54.5 & 0 & 0 & 0 \\
\hline & RT Power Output & 1100 & 1100 & 1100 & 1100 & 1100 & 1100 & 1100 & 1100 & 1100 \\
\hline \multirow{3}{*}{ 醇营 } & DA Power & \multicolumn{3}{|c|}{250} & \multicolumn{3}{|c|}{250} & \multicolumn{3}{|c|}{500} \\
\hline & Regulation Up/Down & -100 & 190 & 250 & 250 & 250 & 250 & -50 & 0 & 0 \\
\hline & RT Power Output & 150 & 440 & 500 & 500 & 500 & 500 & 500 & 450 & 500 \\
\hline \multirow{3}{*}{ 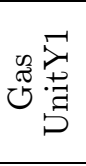 } & DA Power & \multicolumn{3}{|c|}{150} & \multicolumn{3}{|c|}{200} & \multicolumn{3}{|c|}{365} \\
\hline & Regulation Up/Down & -150 & -150 & 0 & -190 & 100 & 200 & -200 & 35 & 35 \\
\hline & RT Power Output & 0 & 0 & 150 & 10 & 300 & 400 & 165 & 400 & 400 \\
\hline \multirow{3}{*}{$\begin{array}{l}\infty^{\infty} \\
\stackrel{\infty}{4} \\
>^{\circ}\end{array}$} & POP & \multicolumn{3}{|c|}{-} & & -364.5 & & & -729 & \\
\hline & Regulation Up/Down & - & - & - & 0 & 0 & +55 & 0 & 5 & 105 \\
\hline & RT Power Output & - & - & - & -364.5 & -364.5 & -309.5 & -729 & -724 & -624 \\
\hline $\begin{array}{l}\infty \\
\infty 0\end{array}$ & $\mathrm{POP}$ & & - & & & 0 & & & -729 & \\
\hline & Regulation Up/Down & - & - & - & -364.5 & -364.5 & -309.5 & 0 & 0 & 110 \\
\hline & RT Power Output & - & - & - & -364.5 & -364.5 & -309.5 & -729 & -729 & -619 \\
\hline
\end{tabular}


Table C.6

The Results of Demand and Generation Dispatch for Case 2

\begin{tabular}{|c|c|c|c|c|c|c|c|c|c|c|}
\hline \multirow{6}{*}{ (5: } & $(\mathrm{MW})$ & \multicolumn{3}{|c|}{ Without EV } & \multicolumn{3}{|c|}{$100,000 \mathrm{EVs}$} & \multicolumn{3}{|c|}{$200,000 \mathrm{EVs}$} \\
\hline & Demand Curtailment of LSE & 0 & 0 & 0 & 0 & 0 & 0 & 0 & 0 & 0 \\
\hline & Total Wind Power Deviation & +250 & -40 & -250 & +250 & -40 & -250 & +250 & -40 & -250 \\
\hline & DA Power & \multicolumn{3}{|c|}{0} & \multicolumn{3}{|c|}{300} & \multicolumn{3}{|c|}{300} \\
\hline & Wind Power Deviation & +200 & -20 & -150 & +200 & -20 & -150 & +200 & -20 & -150 \\
\hline & Wind Power Curtailment & 0 & 0 & 0 & 0 & 0 & 0 & 0 & 0 & 0 \\
\hline \multirow{3}{*}{ 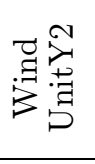 } & DA Power & \multicolumn{3}{|c|}{0} & \multicolumn{3}{|c|}{200} & \multicolumn{3}{|c|}{200} \\
\hline & Wind Power Deviation & +50 & -20 & -100 & +50 & -20 & -100 & +50 & -20 & -100 \\
\hline & Wind Power Curtailment & 0 & 0 & 0 & 0 & 0 & 0 & 0 & 0 & 0 \\
\hline \multirow{3}{*}{ 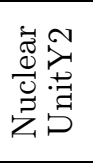 } & DA Power & \multicolumn{3}{|c|}{1500} & \multicolumn{3}{|c|}{1500} & \multicolumn{3}{|c|}{1500} \\
\hline & Regulation Up/Down & 0 & 0 & 0 & 0 & 0 & 0 & 0 & 0 & 0 \\
\hline & RT Power Output & 1500 & 1500 & 1500 & 1500 & 1500 & 1500 & 1500 & 1500 & 1500 \\
\hline \multirow{3}{*}{ 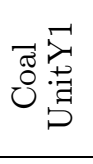 } & DA Power & \multicolumn{3}{|c|}{1100} & \multicolumn{3}{|c|}{1100} & \multicolumn{3}{|c|}{1060} \\
\hline & Regulation Up/Down & -300 & -80 & 0 & 0 & 0 & 0 & 0 & +40 & +40 \\
\hline & RT Power Output & 800 & 1020 & 1100 & 1100 & 1100 & 1100 & 1060 & 1100 & 1100 \\
\hline \multirow{3}{*}{ 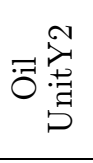 } & DA Power & \multicolumn{3}{|c|}{500} & \multicolumn{3}{|c|}{455} & \multicolumn{3}{|c|}{480} \\
\hline & Regulation Up/Down & -250 & -180 & -100 & -50 & +20 & +45 & -50 & +20 & +20 \\
\hline & RT Power Output & 250 & 320 & 400 & 405 & 475 & 500 & 420 & 500 & 500 \\
\hline \multirow{3}{*}{ שֶ } & DA Power & \multicolumn{3}{|c|}{400} & \multicolumn{3}{|c|}{305} & \multicolumn{3}{|c|}{400} \\
\hline & Regulation Up/Down & -200 & -200 & -150 & -200 & +20 & +95 & -200 & -20 & 0 \\
\hline & RT Power Output & 200 & 200 & 250 & 205 & 325 & 400 & 200 & 380 & 400 \\
\hline \multirow{3}{*}{ 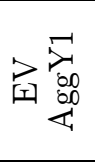 } & POP & \multicolumn{3}{|c|}{-} & \multicolumn{3}{|c|}{-364.5} & & -729 & \\
\hline & Regulation Up/Down & - & - & - & 0 & 0 & +55 & 0 & 0 & +110 \\
\hline & RT Power Output & - & - & - & -364.5 & -364.5 & -309.5 & -729 & -729 & -629 \\
\hline$\stackrel{N}{\Upsilon}$ & $\mathrm{POP}$ & & - & & & -364.5 & & & -729 & \\
\hline $\begin{array}{l}80 \\
800\end{array}$ & Regulation Up/Down & - & - & - & 0 & 0 & +55 & 0 & 0 & +80 \\
\hline & RT Power Output & - & - & - & 364.5 & 364.5 & -309.5 & -729 & -729 & -649 \\
\hline
\end{tabular}


Table C.7

The Results of Demand and Generation Dispatch for Case 3

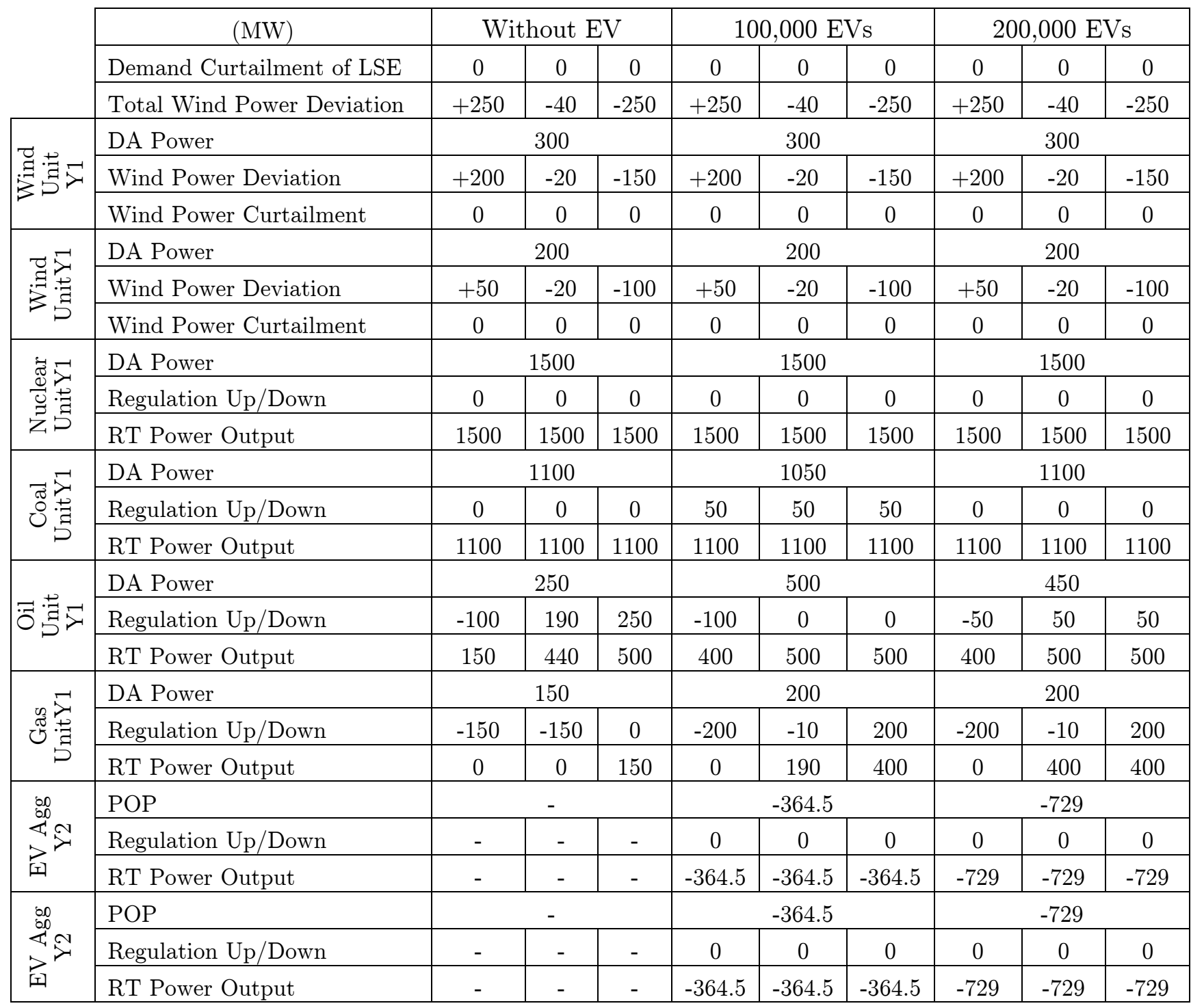


Table C.8

The Results of Demand and Generation Dispatch for Case 4

\begin{tabular}{|c|c|c|c|c|c|c|c|c|c|c|}
\hline \multirow{6}{*}{ 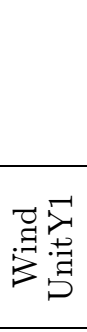 } & $(\mathrm{MW})$ & \multicolumn{3}{|c|}{ Without EV } & \multicolumn{3}{|c|}{$100,000 \mathrm{EVs}$} & \multicolumn{3}{|c|}{$200,000 \mathrm{EVs}$} \\
\hline & Demand Curtailment of LSE & 150 & 40 & 150 & 163.8 & 36.36 & 181.8 & 34.1 & 9.52 & 47.16 \\
\hline & Total Wind Power Deviation & +250 & +10 & -200 & +250 & -40 & -250 & +250 & -40 & -250 \\
\hline & DA Power & \multicolumn{3}{|c|}{300} & \multicolumn{3}{|c|}{300} & \multicolumn{3}{|c|}{300} \\
\hline & Wind Power Deviation & +200 & -20 & -150 & +200 & -20 & -150 & +200 & -20 & -150 \\
\hline & Wind Power Curtailment & 0 & 0 & 0 & 0 & 0 & 0 & 0 & 0 & 0 \\
\hline \multirow{3}{*}{ 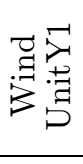 } & DA Power & \multicolumn{3}{|c|}{150} & \multicolumn{3}{|c|}{200} & \multicolumn{3}{|c|}{200} \\
\hline & Wind Power Deviation & +100 & +30 & -50 & +50 & -20 & -100 & +50 & -20 & -100 \\
\hline & Wind Power Curtailment & 0 & 0 & 0 & 0 & 0 & 0 & 0 & 0 & 0 \\
\hline \multirow{3}{*}{ 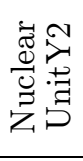 } & DA Power & \multicolumn{3}{|c|}{1500} & \multicolumn{3}{|c|}{1500} & \multicolumn{3}{|c|}{1500} \\
\hline & Regulation Up/Down & 0 & 0 & 0 & 0 & 0 & 0 & 0 & 0 & 0 \\
\hline & RT Power Output & 1500 & 1500 & 1500 & 1500 & 1500 & 1500 & 1500 & 1500 & 1500 \\
\hline \multirow{3}{*}{ 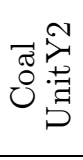 } & DA Power & \multicolumn{3}{|c|}{1100} & \multicolumn{3}{|c|}{1100} & \multicolumn{3}{|c|}{1100} \\
\hline & Regulation Up/Down & 0 & 0 & 0 & 0 & 0 & 0 & 0 & 0 & 0 \\
\hline & RT Power Output & 1100 & 1100 & 1100 & 1100 & 1100 & 1100 & 1100 & 1100 & 1100 \\
\hline \multirow{3}{*}{ 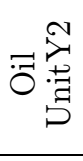 } & DA Power & \multicolumn{3}{|c|}{250} & \multicolumn{3}{|c|}{250} & \multicolumn{3}{|c|}{250} \\
\hline & Regulation Up/Down & -250 & -250 & 250 & -250 & -250 & 250 & -250 & -250 & +250 \\
\hline & RT Power Output & 0 & 0 & 500 & 0 & 0 & 500 & 0 & 0 & 500 \\
\hline \multirow{3}{*}{ 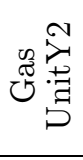 } & DA Power & \multicolumn{3}{|c|}{200} & \multicolumn{3}{|c|}{200} & \multicolumn{3}{|c|}{108} \\
\hline & Regulation Up/Down & -200 & 200 & -200 & -200 & 200 & -200 & -108 & +200 & -108 \\
\hline & RT Power Output & 0 & 400 & 0 & 0 & 400 & 0 & 0 & 308 & 0 \\
\hline \multirow{3}{*}{ 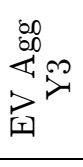 } & $\mathrm{POP}$ & \multicolumn{3}{|c|}{-} & \multicolumn{3}{|c|}{-364.5} & & -729 & \\
\hline & Regulation Up/Down & - & - & - & 36.2 & 16.81 & 0 & 0 & 80.48 & 0 \\
\hline & RT Power Output & - & - & - & -328.3 & -348.3 & -364.5 & -729 & -648.5 & -729 \\
\hline & POP & & - & & & -364.5 & & & -729 & \\
\hline$>^{4}$ & Regulation Up/Down & - & - & - & 0 & 36.83 & 18.2 & 73.9 & 0 & 60.84 \\
\hline 国 & RT Power Output & - & - & - & -364.5 & -327.6 & -346.3 & -655.1 & -729 & -668.1 \\
\hline
\end{tabular}





\section{Bibliography}

[1] M. H. K. Tushar, C. Assi, M. Maier, and M. F. Uddin, "Smart Microgrids: Optimal Joint Scheduling for Electric Vehicles and Home Appliances", IEEE Trans. Smart Grid, vol.5, no.1, pp.239-250, Jan. 2014.

[2] C. Guille and G. Gross, "Design of a Conceptual Framework for the V2G Implementation," IEEE Energy 2030 Conference, vol., no., pp.1-3, 17-18 Nov. 2008.

[3] Frankfurt School-UNEP Centre/BNEF, "Global Trends in Renewable Energy Investment 2014”, [Online] Available: http://www.unep.org/pdf/Green ' energy`2013-Key findings.pdf.

[4] International Energy Agency, "Global EV Outlook" [Online] Available:https://www.iea.org/publications/globalevoutlook'2013.pdf.

[5] Global Wind energy Council, "Global Wind Energy Outlook 2014" [Online] Available: http:// www.gwec.net/wp-content/ uploads/2014/10/ GWEO2014'WEB.pdf.

[6] A. Brooks, E. Lu, D. Reicher, C. Spirakis, and B. Weihl, "Demand Dispatch", IEEE Power and Energy Magazine, vol.8, no.3, pp.20,29, May-Jun. 2010.

[7] A. T. Al-Awami, and E. Sortomme, "Coordinating Vehicle-to-Grid Services With Energy Trading", IEEE Trans. Smart Grid, vol.3, no.1, pp.453-462, Mar. 2012.

[8] E. Sortomme, and M. A. El-Sharkawi, "Optimal Charging Strategies for Unidirectional Vehicle-to-Grid", IEEE Trans. Smart Grid, vol.2, no.1, pp.131-138, Mar. 2011. 
[9] Sortomme, E.; El-Sharkawi, M.A., "Optimal Scheduling of Vehicle-toGrid Energy and Ancillary Services", IEEE Trans. Smart Grid, vol.3, no.1, pp.351-359, Mar. 2012.

[10] A. Rogers, A. Henderson, X. Wang, and M. Negnevitsky, "Compressed air energy storage: Thermodynamic and economic review," PES General Meeting, 2014 IEEE Conference \& Exposition, vol., no., pp.1, 5, 27-31 Jul. 2014.

[11] M. Shafie-khah, J.P.S. Catalao, "A Stochastic Multi-Layer Agent-Based Model to Study Electricity Market Participants Behavior," IEEE Trans. Power Syst., vol.30, no.2, pp.867-881, March 2015.

[12] L. V. L. Abreu, M. E. Khodayar, M. Shahidehpour, and Lei Wu, "RiskConstrained Coordination of Cascaded Hydro Units with Variable Wind Power Generation," IEEE Trans. Sustain. Energy, vol.3, no.3, pp.359368, Jul. 2012.

[13] S. J. Kazempour, H. Zareipour, "Equilibria in an Oligopolistic Market With Wind Power Production," IEEE Trans. Power Syst., vol.29, no.2, pp.686,697, March 2014.

[14] J. G. Vlachogiannis, "Probabilistic Constrained Load Flow Considering Integration of Wind Power Generation and Electric Vehicles," in IEEE Transactions on Power Systems, vol. 24, no. 4, pp. 1808-1817, Nov. 2009.

[15] G. Li and X. P. Zhang, "Modeling of Plug-in Hybrid Electric Vehicle Charging Demand in Probabilistic Power Flow Calculations," in IEEE Transactions on Smart Grid, vol. 3, no. 1, pp. 492-499, March 2012.

[16] Y. Ma, T. Houghton, A. Cruden and D. Infield, "Modeling the Benefits of Vehicle-to-Grid Technology to a Power System," in IEEE Transactions on Power Systems, vol. 27, no. 2, pp. 1012-1020, May 2012.

[17] Z. Darabi and M. Ferdowsi, "Aggregated Impact of Plug-in Hybrid Electric Vehicles on Electricity Demand Profile," in IEEE Transactions on Sustainable Energy, vol. 2, no. 4, pp. 501-508, Oct. 2011.

[18] A. Ashtari, E. Bibeau, S. Shahidinejad and T. Molinski, "PEV Charging Profile Prediction and Analysis Based on Vehicle Usage Data," in IEEE Transactions on Smart Grid, vol. 3, no. 1, pp. 341-350, March 2012.

[19] S. Shao, M. Pipattanasomporn and S. Rahman, "Grid Integration of Electric Vehicles and Demand Response With Customer Choice," in IEEE Transactions on Smart Grid, vol. 3, no. 1, pp. 543-550, March 2012. 
[20] S. Shahidinejad, S. Filizadeh and E. Bibeau, "Profile of Charging Load on the Grid Due to Plug-in Vehicles," in IEEE Transactions on Smart Grid, vol. 3, no. 1, pp. 135-141, March 2012.

[21] J. R. Pillai and B. Bak-Jensen, "Integration of Vehicle-to-Grid in the Western Danish Power System," in IEEE Transactions on Sustainable Energy, vol. 2, no. 1, pp. 12-19, Jan. 2011.

[22] S. Deilami, A. S. Masoum, P. S. Moses and M. A. S. Masoum, "RealTime Coordination of Plug-In Electric Vehicle Charging in Smart Grids to Minimize Power Losses and Improve Voltage Profile," in IEEE Transactions on Smart Grid, vol. 2, no. 3, pp. 456-467, Sept. 2011.

[23] E. Sortomme, M. M. Hindi, S. D. J. MacPherson and S. S. Venkata, "Coordinated Charging of Plug-In Hybrid Electric Vehicles to Minimize Distribution System Losses," in IEEE Transactions on Smart Grid, vol. 2, no. 1, pp. 198-205, March 2011.

[24] H. Turker, S. Bacha, D. Chatroux and A. Hably, "Low-Voltage Transformer Loss-of-Life Assessments for a High Penetration of Plug-In Hybrid Electric Vehicles (PHEVs)," in IEEE Transactions on Power Delivery, vol. 27, no. 3, pp. 1323-1331, July 2012.

[25] K. Clement-Nyns, E. Haesen and J. Driesen, "The Impact of Charging Plug-In Hybrid Electric Vehicles on a Residential Distribution Grid," in IEEE Transactions on Power Systems, vol. 25, no. 1, pp. 371-380, Feb. 2010.

[26] L. Pieltain Fernandez, T. Gomez San Roman, R. Cossent, C. Mateo Domingo and P. Frias, "Assessment of the Impact of Plug-in Electric Vehicles on Distribution Networks," in IEEE Transactions on Power Systems, vol. 26, no. 1, pp. 206-213, Feb. 2011.

[27] Z. Liu, F. Wen and G. Ledwich, "Optimal Planning of Electric-Vehicle Charging Stations in Distribution Systems," in IEEE Transactions on Power Delivery, vol. 28, no. 1, pp. 102-110, Jan. 2013.

[28] M. Etezadi-Amoli, K. Choma and J. Stefani, "Rapid-Charge ElectricVehicle Stations," in IEEE Transactions on Power Delivery, vol. 25, no. 3, pp. 1883-1887, July 2010.

[29] W. Su and M. Y. Chow, "Performance Evaluation of an EDA-Based Large-Scale Plug-In Hybrid Electric Vehicle Charging Algorithm," in IEEE Transactions on Smart Grid, vol. 3, no. 1, pp. 308-315, March 2012. 
[30] F. Guo, E. Inoa, W. Choi and J. Wang, "Study on Global Optimization and Control Strategy Development for a PHEV Charging Facility," in IEEE Transactions on Vehicular Technology, vol. 61, no. 6, pp. 24312441, July 2012.

[31] M. Singh, P. Kumar and I. Kar, "A Multi Charging Station for Electric Vehicles and Its Utilization for Load Management and the Grid Support," in IEEE Transactions on Smart Grid, vol. 4, no. 2, pp. 10261037, June 2013.

[32] Z. Liu, F. Wen and G. Ledwich, "Optimal Siting and Sizing of Distributed Generators in Distribution Systems Considering Uncertainties," in IEEE Transactions on Power Delivery, vol. 26, no. 4, pp. 2541-2551, Oct. 2011.

[33] A. Mohsenzadeh, C. Pang, S. Pazouki and M. Haghifam, "Optimal siting and sizing of electric vehicle public charging stations considering smart distribution network reliability," North American Power Symposium (NAPS), 2015, Charlotte, NC, 2015, pp. 1-6.

[34] L. Jia, Z. Hu, Y. Song and Z. Luo, "Optimal siting and sizing of electric vehicle charging stations," Electric Vehicle Conference (IEVC), 2012 IEEE International, Greenville, SC, 2012, pp. 1-6.

[35] M. E. Khodayar, L. Wu and M. Shahidehpour, "Hourly Coordination of Electric Vehicle Operation and Volatile Wind Power Generation in SCUC," in IEEE Transactions on Smart Grid, vol. 3, no. 3, pp. 12711279, Sept. 2012.

[36] P. Mitra and G. K. Venayagamoorthy, "Wide area control for improving stability of a power system with plug-in electric vehicles," in IET Generation, Transmission \& Distribution, vol. 4, no. 10, pp. 1151-1163, October 2010.

[37] Z. Darabi and M. Ferdowsi, "Aggregated Impact of Plug-in Hybrid Electric Vehicles on Electricity Demand Profile," in IEEE Transactions on Sustainable Energy, vol. 2, no. 4, pp. 501-508, Oct. 2011.

[38] M. D. Galus, S. Koch and G. Andersson, "Provision of Load Frequency Control by PHEVs, Controllable Loads, and a Cogeneration Unit," in IEEE Transactions on Industrial Electronics, vol. 58, no. 10, pp. 45684582, Oct. 2011. 
[39] J. Tomic and W. Kempton, "Using fleets of electric-drive vehicles for grid support," Journal of Power Sources, 168(2), pp. 459-468., Jun. 2007.

[40] M. E. Khodayar, L. Wu and Z. Li, "Electric Vehicle Mobility in Transmission-Constrained Hourly Power Generation Scheduling," in IEEE Transactions on Smart Grid, vol. 4, no. 2, pp. 779-788, June 2013.

[41] E. Sortomme and M. A. El-Sharkawi, "Optimal Combined Bidding of Vehicle-to-Grid Ancillary Services," in IEEE Transactions on Smart Grid, vol. 3, no. 1, pp. 70-79, March 2012.

[42] J. Tomic and W. Kempton, "Vehicle-to-grid power implementation: From stabilizing the grid to supporting large-scale renewable energy," Journal of power sources, 144(1), pp. 280-294, Jun 2005.

[43] J. Tomic and W. Kempton, "Vehicle-to-grid power fundamentals: Calculating capacity and net revenue," J Journal of power sources, 144 (1), pp. 268-279, Jun 2005.

[44] A. Kavousi-Fard, T. Niknam and M. Fotuhi-Firuzabad, "Stochastic Reconfiguration and Optimal Coordination of V2G Plug-in Electric Vehicles Considering Correlated Wind Power Generation," in IEEE Transactions on Sustainable Energy, vol. 6, no. 3, pp. 822-830, July 2015.

[45] Z. Li, Q. Guo, H. Sun, Y. Wang and S. Xin, "Emission-Concerned WindEV Coordination on the Transmission Grid Side With Network Constraints: Concept and Case Study," in IEEE Transactions on Smart Grid, vol. 4, no. 3, pp. 1692-1704, Sept. 2013.

[46] W. Hu, C. Su, Z. Chen and B. Bak-Jensen, "Optimal Operation of PlugIn Electric Vehicles in Power Systems With High Wind Power Penetrations," in IEEE Transactions on Sustainable Energy, vol. 4, no. 3, pp. 577-585, July 2013.

[47] H. N. T. Nguyen, C. Zhang and M. A. Mahmud, "Optimal Coordination of G2V and V2G to Support Power Grids With High Penetration of Renewable Energy," in IEEE Transactions on Transportation Electrification, vol. 1, no. 2, pp. 188-195, Aug. 2015.

[48] S. Gao, K. T. Chau, C. Liu, D. Wu and C. C. Chan, "Integrated Energy Management of Plug-in Electric Vehicles in Power Grid With Renewables," in IEEE Transactions on Vehicular Technology, vol. 63, no. 7, pp. 3019-3027, Sept. 2014. 
[49] Z. Li, Q. Guo, H. Sun, Y. Wang and S. Xin, "Emission-Concerned WindEV Coordination on the Transmission Grid Side With Network Constraints: Concept and Case Study," in IEEE Transactions on Smart Grid, vol. 4, no. 3, pp. 1692-1704, Sept. 2013.

[50] L. Goransson, S. Karlsson, and F. Johnsson, "Integration of plug-in hybrid electric vehicles in a regional wind-thermal power system," J. Energy Policy, vol. 38, no. 10, pp. 5482-5492, Oct. 2010

[51] Liu Cong, W. Jianhui A. Botterud, Yan Zhou, and A. Vyas, "Assessment of Impacts of PHEV Charging Patterns on Wind-Thermal Scheduling by Stochastic Unit Commitment", IEEE Trans. Smart Grid, vol.3, no.2, pp.675-683, Jun. 2012.

[52] Wu Ting, Yang Qiang, Bao Zhejing, Yan Wenjun, "Coordinated Energy Dispatching in Microgrid With Wind Power Generation and Plug-in Electric Vehicles", IEEE Trans. Smart Grid, vol.4, no.3, pp.1453-1463, Sep. 2013.

[53] S. I. Vagropoulos, and A. G. Bakirtzis, , "Optimal Bidding Strategy for Electric Vehicle Aggregators in Electricity Markets", IEEE Trans. Power Syst., vol.28, no.4, pp.4031-4041, Nov. 2013.

[54] L. Baringo and A. J. Conejo, "Strategic offering for a wind power producer," IEEE Trans. Power Syst., vol.28, no.4, pp.4645, 4654, Nov. 2013.

[55] M. Zugno, J. M. Morales, P. Pinson, and H. Madsen, "Pool Strategy of a Price-Maker Wind Power Producer," IEEE Trans. Power Syst., vol.28, no.3, pp.3440, 3450, Aug. 2013.

[56] M. Gonzalez Vaya, G. Andersson, "Optimal Bidding Strategy of a PlugIn Electric Vehicle Aggregator in Day-Ahead Electricity Markets Under Uncertainty," IEEE Trans. Power Syst., 2015, IEEE early access articles.

[57] Conejo, Antonio J., Miguel Carrión, and Juan M. Morales. "Decision making under uncertainty in electricity markets". Vol. 1. New York: Springer, 2010.

[58] A. T. Al-Awami and M. A. El-Sharkawi, "Coordinated Trading of Wind and Thermal Energy," in IEEE Transactions on Sustainable Energy, vol. 2, no. 3, pp. 277-287, July 2011. 
[59] Li Tao, M. Shahidehpour, and Li Zuyi, "Risk-Constrained Bidding Strategy With Stochastic Unit Commitment," IEEE Trans. Power Syst., vol.22, no.1, pp.449,458, Feb. 2007.

[60] M. A. Plazas, A. J. Conejo, and F. Prieto, "Multimarket optimal bidding for a power producer," IEEE Trans. Power Syst., vol. 20, no. 4, pp. 20412050, Nov. 2005.

[61] S Mahir. "Modeling the Diversification Benefit of Transmission Investments." Master thesis, Second Level, Univ. KTH, Sweden, 2012

[62] N. Amjady, J. Aghaei, and H. A. Shayanfar, "Stochastic Multiobjective Market Clearing of Joint Energy and Reserves Auctions Ensuring Power System Security", IEEE Trans. Power Syst., vol.24, no.4, pp.1841-1854, Nov. 2009.

[63] L. Soder, "Simulation of wind speed forecast errors for operation planning of multiarea power systems," in Proc. 2004 Int. Conf. Proc. Probabilistic Methods Applied to Power Systems, Sep. 12-16, 2004, pp. 723-728.

[64] A. Boone, "Simulation of Short-Term Wind Speed Forecast Errors Using a Multi-Variate ARMA (1,1) Time-Series Model," Master thesis, Univ. KTH Royal Institute of Technology, Sweden, 2005.

[65] J. M. Morales, A. J. Conejo, and J. Perez-Ruiz, "Short-term trading for a wind power producer," IEEE Trans. Power Syst., vol. 25, no. 1, pp. 554564, Feb. 2010.

[66] Electrification Coalition, "Electrification roadmap: Revolutionizing transportation and achieving energy security," Nov. 2013 [Online]. Available:http://www.electrificationcoalition.org/sites/default/files/SAF 1213EC-Roadmap 'v12Online.pdf.

[67] J. Dupacova , N. Growe-Kuska, and W. Romisch, "Scenario reduction in stochastic programming: an approach using probability metrics," Math. Prog. Series A, vol. 3, pp. 493-511, 2003.

[68] N. Growe-Kuska, H. Heitsch, and W. Romisch, "Scenario reduction and scenario tree construction for power management problems," in Proc. IEEE Power Tech Conf., Bologna, Italy, Jun. 2003, vol. 3, pp. 23-26. 
[69] GAMS/SCENRED Documentation [Online]. Available: http://www.gams.com/docs/document.htm.

[70] Stoft, S. "Power System Economics: Designing Markets for Electricity, 2002.

[71] Gabriel, Steven A., Antonio J. Conejo, J. David Fuller, Benjamin F. Hobbs, and Carlos Ruiz. Complementarity modeling in energy markets. Vol. 180. Springer, 2012.

[72] Day, Christopher J., Benjamin F. Hobbs, and J-S. Pang. "Oligopolistic competition in power networks: a conjectured supply function approach." Power Systems, IEEE Transactions on 17.3 (2002): 597-607.

[73] Farahat, A., and Georgia P. "Technical note-a comparison of bertrand and cournot profits in oligopolies with differentiated products." Operations research 59.2, 507-513 2011.

[74] Hobbs, Benjamin F. "Network models of spatial oligopoly with an application to deregulation of electricity generation." Operations Research 34.3, 395-409, 1986.

[75] Kwang-Ho Lee and R. Baldick, "Solving three-player games by the matrix approach with application to an electric power market," in IEEE Transactions on Power Systems, vol. 18, no. 4, pp. 1573-1580, Nov. 2003.

[76] Morrow, W. Ross, and Steven J. Skerlos. "Fixed-point approaches to computing Bertrand-Nash equilibrium prices under mixed-logit demand." Operations research 59.2, 328-345, 2011.

[77] Hobbs, Benjamin F., and Fieke AM Rijkers. "Strategic generation with conjectured transmission price responses in a mixed transmission pricing system-Part I: formulation." Power Systems, IEEE Transactions on 19.2 (2004): 707-717.

[78] D. Chattopadhyay, "Multicommodity spatial Cournot model for generator bidding analysis," in IEEE Transactions on Power Systems, vol. 19, no. 1, pp. 267-275, Feb. 2004.

[79] L. B. Cunningham, R. Baldick and M. L. Baughman, "An empirical study of applied game theory: transmission constrained Cournot behavior," in IEEE Transactions on Power Systems, vol. 17, no. 1, pp. 166-172, Feb 2002. 
[80] V. P. Gountis and A. G. Bakirtzis, "Efficient determination of Cournot equilibria in electricity markets," in IEEE Transactions on Power Systems, vol. 19, no. 4, pp. 1837-1844, Nov. 2004.

[81] Metzler, Carolyn, Benjamin F. Hobbs, and Jong-Shi Pang. "NashCournot equilibria in power markets on a linearized DC network with arbitrage: Formulations and properties." Networks and Spatial Economics 3.2, 123-150, 2003.

[82] U. Helman and B. F. Hobbs, "Large-Scale Market Power Modeling: Analysis of the U.S. Eastern Interconnection and Regulatory Applications," in IEEE Transactions on Power Systems, vol. 25, no. 3, pp. 1434-1448, Aug. 2010.

[83] Anderson, Edward J., and Andrew B. Philpott. "Using supply functions for offering generation into an electricity market." Operations Research 50.3, 477-489, 2002.

[84] Anderson, Edward J., and Huifu Xu. "Supply function equilibrium in electricity spot markets with contracts and price caps." Journal of Optimization Theory and Applications 124.2, 257-283, 2005.

[85] Baldick, Ross, Ryan Grant, and Edward Kahn. "Theory and application of linear supply function equilibrium in electricity markets." Journal of regulatory economics 25.2, 143-167, 2004.

[86] Green, Richard J., and David M. Newbery. "Competition in the British electricity spot market." Journal of political economy, 929-953, 1992.

[87] E. Hasan, F. D. Galiana and A. J. Conejo, "Electricity Markets Cleared by Merit Order-Part I: Finding the Market Outcomes Supported by Pure Strategy Nash Equilibria," in IEEE Transactions on Power Systems, vol. 23, no. 2, pp. 361-371, May 2008.

[88] C. Ruiz, A. J. Conejo and Y. Smeers, "Equilibria in an Oligopolistic Electricity Pool With Stepwise Offer Curves," in IEEE Transactions on Power Systems, vol. 27, no. 2, pp. 752-761, May 2012.

[89] M. O. Buygi, H. Zareipour and W. D. Rosehart, "Impacts of Large-Scale Integration of Intermittent Resources on Electricity Markets: A Supply Function Equilibrium Approach," in IEEE Systems Journal, vol. 6, no. 2, pp. 220-232, June 2012. 
[90] J. Fortuny-Amat and B. McCarl, "A representation and economic interpretation of a two-level programming problem," J. Oper. Res. Soc., vol. 32, no. 9, pp. 783-792, Sep. 1981.

[91] J. Kazempour "Strategic Generation Investment and Equilibria in Oligopolistic Electricity Markets," Thesis Doctoral, Ciudad Real, Mayo de 2013.

[92] H. D. Sherali, "A multiple leader Stackelberg model and analysis," Oper. Res., vol. 32, pp. 390-404, Mar. 1984.

[93] C. Ruiz and A. J. Conejo, "Pool Strategy of a Producer With Endogenous Formation of Locational Marginal Prices," in IEEE Transactions on Power Systems, vol. 24, no. 4, pp. 1855-1866, Nov. 2009.

[94] E. Hasan, F. Galiana, and A. J. Conejo, "Electricity markets cleared by merit order, part I: Finding the market outcomes supported by pure strategy Nash equilibria," IEEE Trans. Power Syst., vol. 23, no. 2, pp. 361-371, May 2008.

[95] X. Hu and D. Ralph, "Using EPECs to model bilevel games in restructured electricity markets with locational prices," Oper. Res., vol. 55, pp. 809-827, Sep. 2007.

[96] B. F. Hobbs, C. B. Metzler, and J.-S. Pang, "Strategic gaming analysis for electric power systems: An MPEC approach," IEEE Trans. Power Syst., vol. 15, no. 2, pp. 638-645, May 2000.

[97] A. Tavakoli, M. Negnevitsky, D. T. Nguyen, K. Muttaqi, "Energy Exchange between Electric Vehicle Load and Wind Generating Utilities", IEEE Transactions on Power Systems, vol. 31, no. 2, pp. 1248-1258, March 2016.

[98] A. Tavakoli, M. Negnevitsky, K. Muttaqi," A Coordinated Approach to Energy Exchange between Electric Vehicle Load Aggregators and Wind Generation Companies under Uncertainty", 2015 IEEE Power \& Energy Society General Meeting, Denver, CO, 2015, pp. 1-5. 
[99] J. M. Morales, A. J. Conejo, K. Liu, and J. Zhong, "Pricing electricityin pools with wind producers," IEEE Trans. Power Syst., vol. 23, no. 3, pp. 1366-1376, Aug. 2012.

[100] Tavakoli, M. Negnevitsky, K. Muttaqi," Pool Strategy of a Producer Coordinated with Vehicle-to-Grid Services to Maximize Profitability", Power Engineering Conference (AUPEC), 2015 Australasian Universities, Wollongong, NSW, 2015, pp. 1-6.

[101] M. E. Khodayar, and M. Shahidehpour, "Stochastic Price-Based Coordination of Intrahour Wind Energy and Storage in a Generation Company", IEEE Trans. Sustain. Energy, vol.4, no.3, pp.554-562, Jul. 2013.

[102] J. Contreras, R. Espinola, F. J. Nogales, A. J. Conejo, "ARIMA models to predict next-day electricity prices," IEEE Trans. Power Syst., vol.18, no.3, pp.1014,1020, Aug. 2003 